\title{
Mercury Chalcogenide Quantum Dots: Material Perspective for Device Integration
}

\section{Charlie Gréboval ${ }^{1}$, Audrey Chu ${ }^{1}$, Nicolas Goubet ${ }^{2}$, Clément Livache ${ }^{1}$, Sandrine Ithurria ${ }^{3}$, Emmanuel Lhuillier ${ }^{*}$}

${ }^{1}$ Sorbonne Université, CNRS, Institut des NanoSciences de Paris, INSP, F-75005 Paris, France

${ }^{2}$ Sorbonne Université, CNRS, Laboratoire de la Molécule aux Nano-objets; Réactivité, Interactions et Spectroscopies, MONARIS, F-75005 Paris, France

${ }^{3}$ Laboratoire de Physique et d'Etude des Matériaux, ESPCI-Paris, PSL Research University, Sorbonne Université Univ Paris 06, CNRS UMR 8213, 10 rue Vauquelin 75005 Paris, France.

* To whom correspondence should be sent: el@insp.upmc.fr

\begin{abstract}
Nanocrystals (NCs) are one of the few nanotechnologies to have attained mass market applications with their use as light sources for displays. This success relies on Cd- and In-based wide bandgap materials. NCs are likely to be employed in more applications as they provide a versatile platform for optoelectronics, specifically, infrared optoelectronics. The existing material technologies in this range of wavelengths are generally not cost effective, which limits the spread of technologies beyond a few niche domains, such as defense and astronomy. Among the potential candidates to address the infrared window, mercury chalcogenide $(\mathrm{HgX}) \mathrm{NCs}$ exhibit the highest potential in terms of performance. In this review, we discuss how material developments have facilitated device enhancements. Because such NCs are primarily used because of their infrared optical features, we first review the strategies for the associated colloidal growth and electronic structure. The review is organized considering three main device-related applications: light emission, electronic transport and infrared photodetection.
\end{abstract}

Keywords: nanocrystals, infrared, mercury chalcogenides, optoelectronics, light detection.

Graphic for the table of contents:

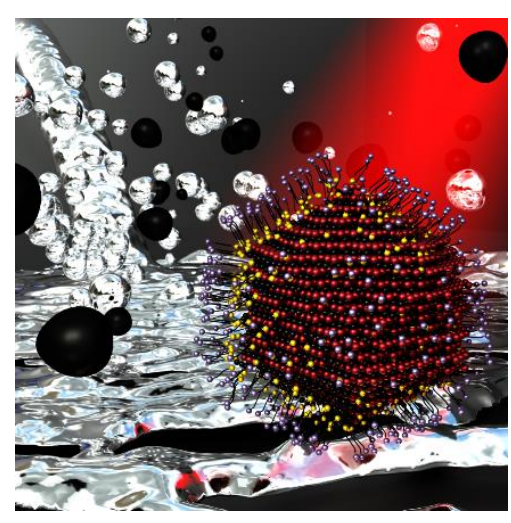


1. INTRODUCTION.

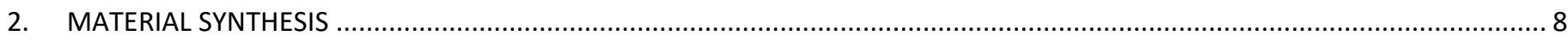

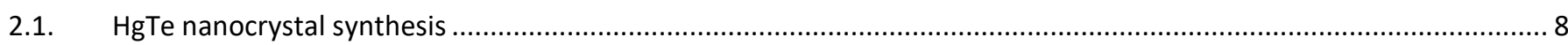

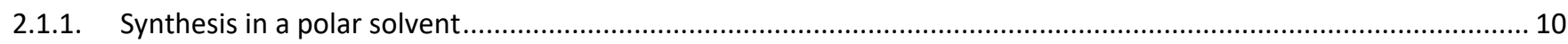

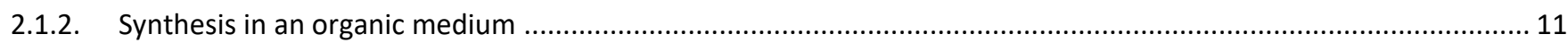

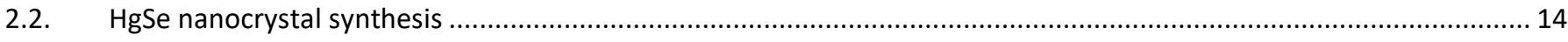

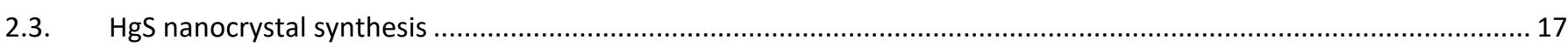

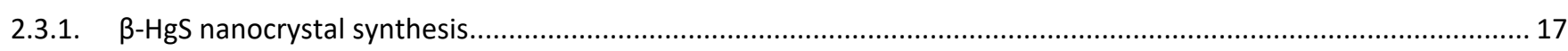

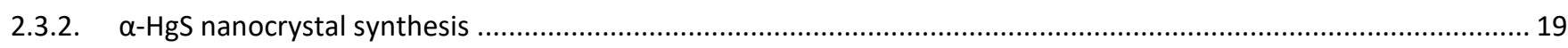

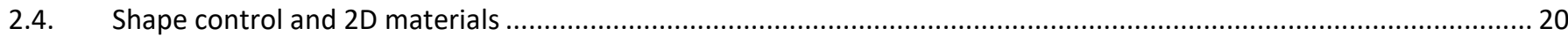

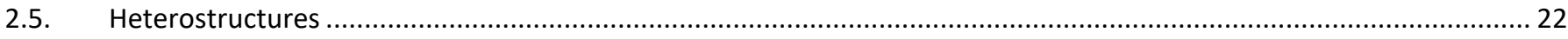

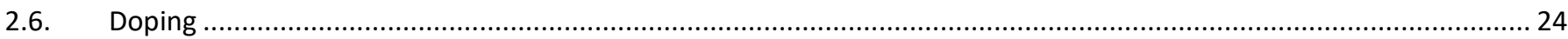

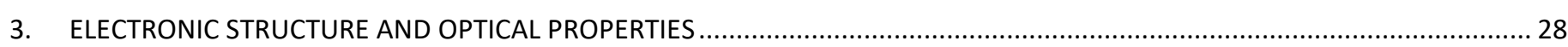

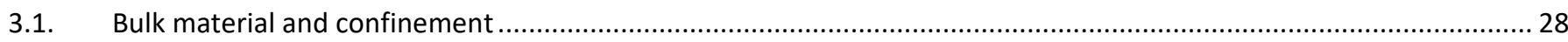

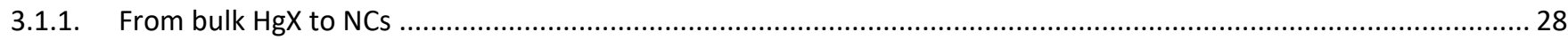

3.1.2. Effective mass approximation and multiband k.p model .................................................................................... 29

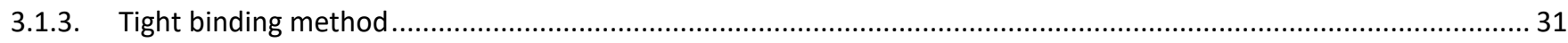

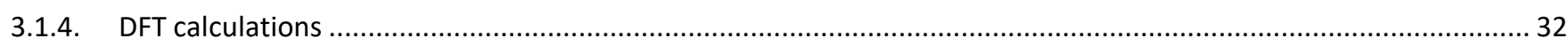

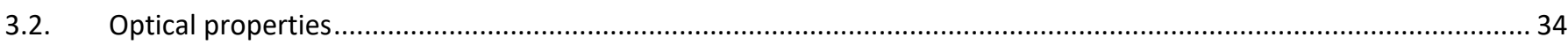

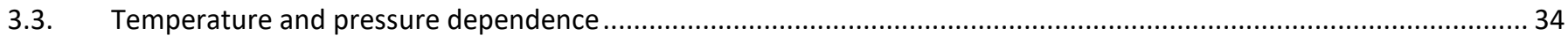

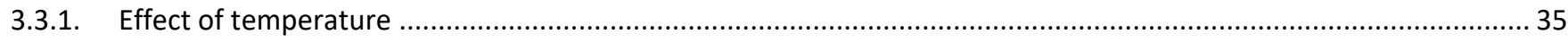

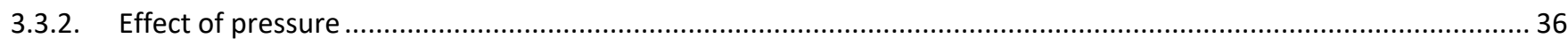

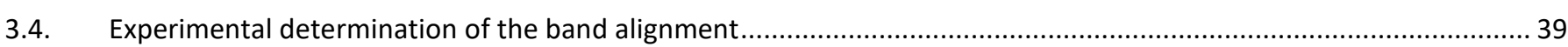

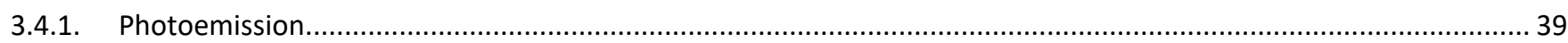

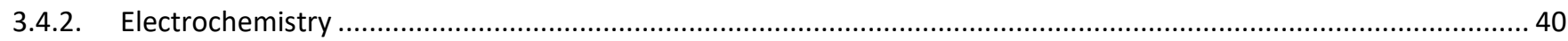

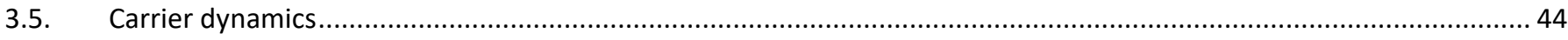

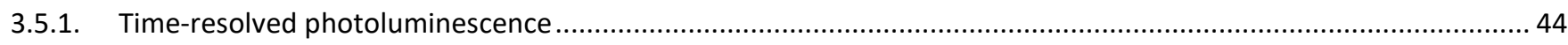

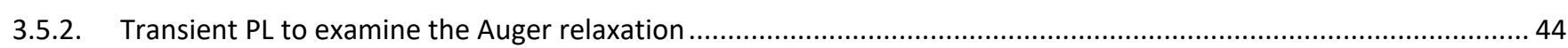

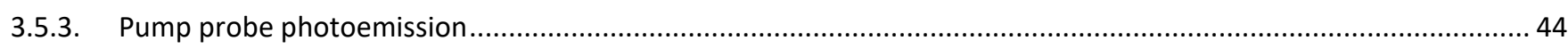

3.5.4. Transient photocurrent to examine the trap distribution ............................................................................... 46

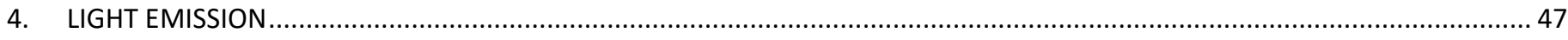

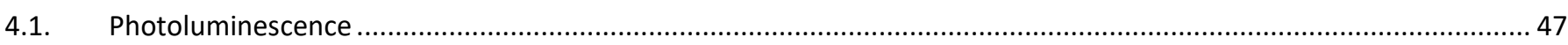

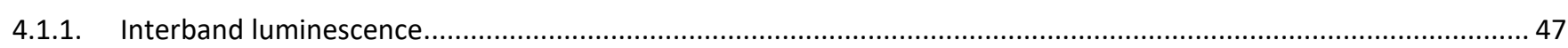

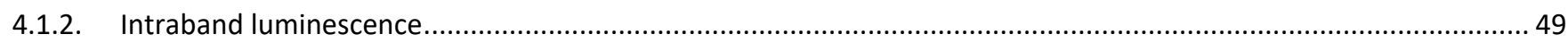

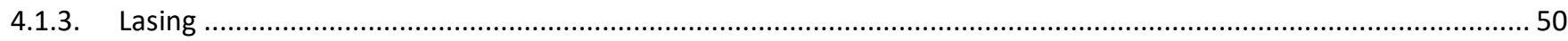

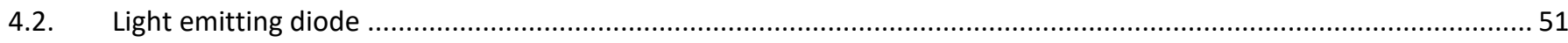

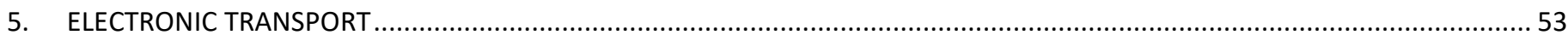

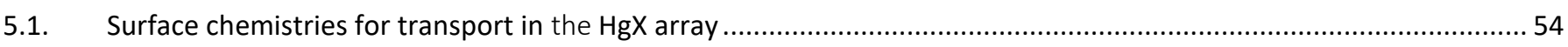

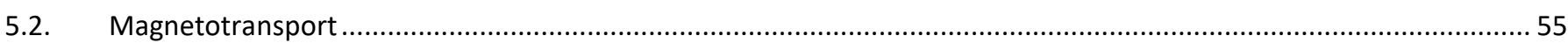




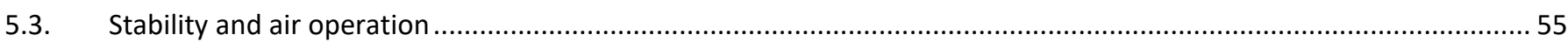

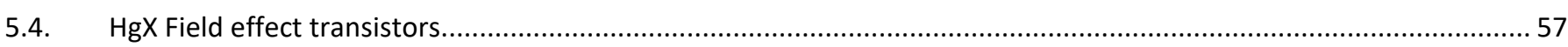

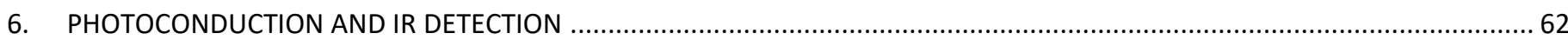

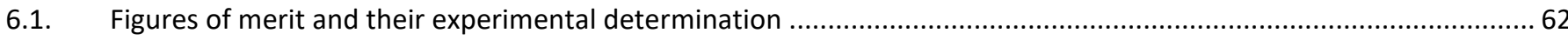

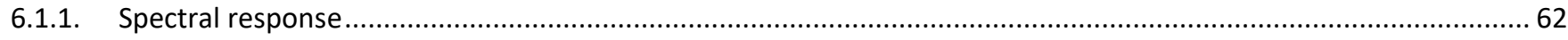

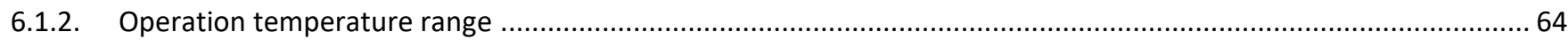

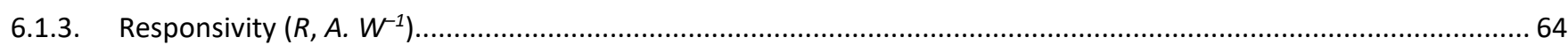

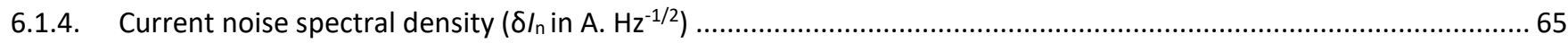

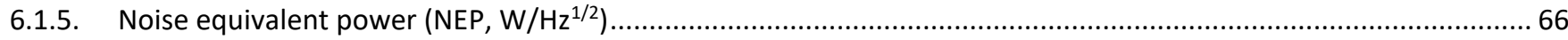

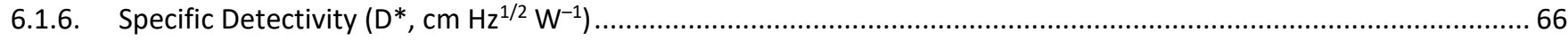

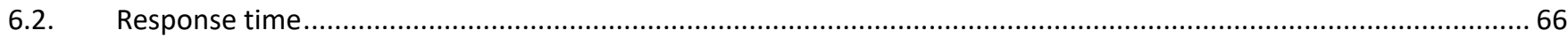

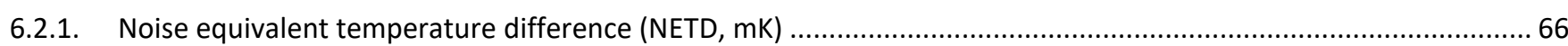

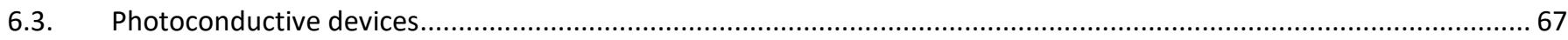

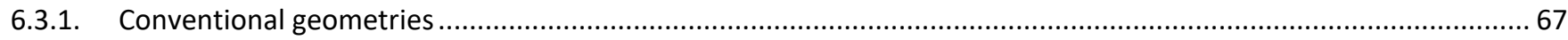

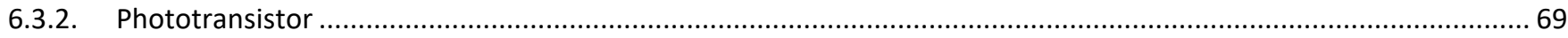

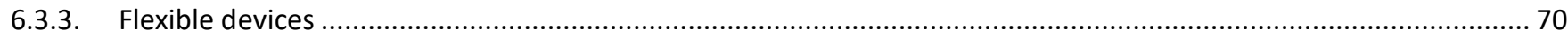

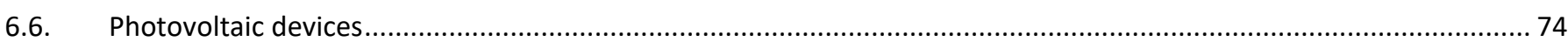

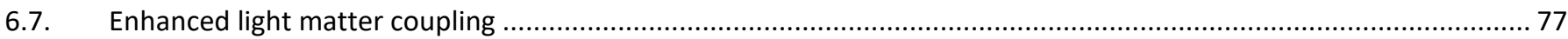

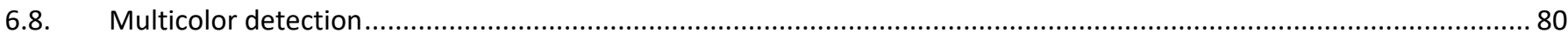

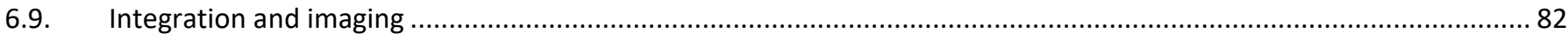

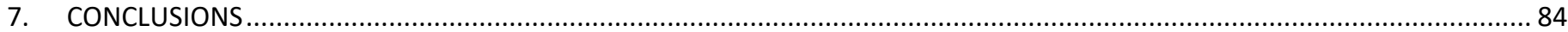

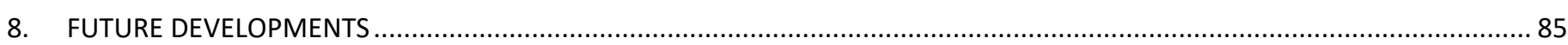

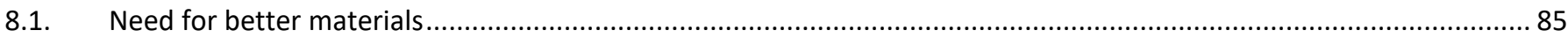

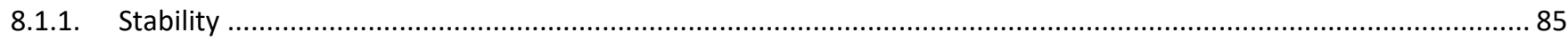

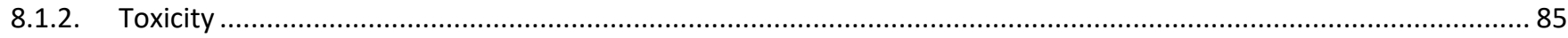

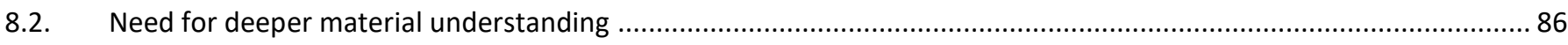

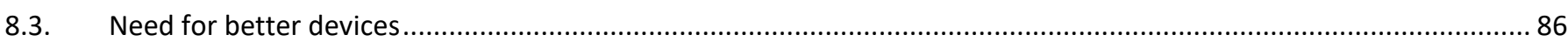

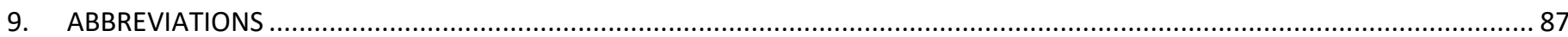

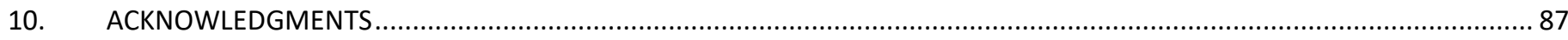

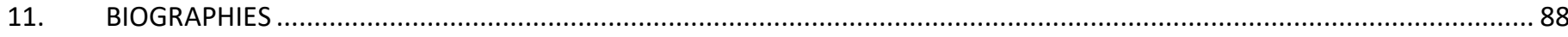

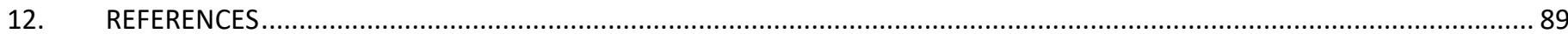




\section{INTRODUCTION}

This review is related to the merging of two fields, specifically, infrared (IR) sensing, in which mercury chalcogenides have been widely applied (see Figure 1), and colloidal semiconductor nanocrystals (NCs), which are nanometer sized crystals of semiconductors. For a long period of time, these two fields evolved independently, and they started to merge only 10 y ago ${ }^{1-3}$ owing to the increase in the cost constraints of IR detection and the maturity of the growth and material processing techniques of NCs (toward conductive films). ${ }^{4}$

The infrared range can enable individuals to see in the dark in a fully passive manner (seeing without being seen) through thermal imaging. For wavelengths above $3 \mu \mathrm{m}$, the blackbody emission signal starts to prevail over reflection from external sources, owing to which the objects and bodies become primary light sources, and the emitted radiation from these objects can be observed with a suitable (i.e., wavelength matched) detector.

The first IR sensors that emerged in the 1950s were mostly based on lead chalcogenide materials, whose bulk bandgap was excessively large to realize thermal detection, as shown in Figure 1. Many candidate materials were investigated, and InSb, among the emerging III-V semiconductors, appeared to be one of the most promising material for detection in the 3-5 $\mu \mathrm{m}$ range (midwave infrared: MWIR). Nevertheless, the actual targeted spectral range was 8-12 $\mu \mathrm{m}$, which matched the maximum emission for a blackbody at room temperature as well as the atmospheric transparency window. This spectral range corresponds to an energy transition of $120 \mathrm{meV}$, requiring very narrow bandgap semiconductors. Moreover, a series of materials, including gray tin, HgTe and $\mathrm{HgSe}$, were investigated; however, these materials appeared to be semimetallic in their bulk form, resulting in inferior or nonexistent photoconductive properties. In 1959, $\mathrm{HgCdTe}$ alloys ${ }^{5}$ emerged as photoconductive materials with a performance comparable to that of InSb in the MWIR. In such alloys, bandgap tuning could be realized by adjusting the $\mathrm{Cd}$ to $\mathrm{Hg}$ ratio. With a $20 \%$ content of $\mathrm{Cd}$, the cutoff wavelength of $\mathrm{HgCdTe}$ was approximately $12 \mu \mathrm{m}$, and the material exhibited a reasonable performance for low temperature operation. Thus, the development of $\mathrm{HgCdTe}$ was driven by its application (ie passive imaging for military). At present, the motivation for this early application may appear paradoxical since HgTe/CdTe quantum well structures regained interest in academic laboratories owing to their specific electronic structure that makes such materials topological insulators. Nevertheless, this military driven application has rendered $\mathrm{HgCdTe}$ as one of the most investigated semiconductors, even if it notably lags Si and GaAs. To date, in the family of infrared semiconductors (InGaAs, InSb, HgCdTe) and heterostructures (QWIP and type II superlattices), HgCdTe remains the leading material for IR (3-12 $\mu \mathrm{m})$ detection, offering a wide spectral range tunability from visible to longwave infrared, while always matching the highest performance for each spectral range.

Since 1993, monodisperse syntheses of colloidal NCs are introduced through the hot precursor injection method. ${ }^{6}$ This process allows the solution growth of semiconductor particles to generate quantum confined materials with an atom like spectrum. For a long time, colloidal syntheses have experienced a lack of conviction from the semiconductor community, owing to the lower control and carrier mobilities associated with these approaches than those attained using conventional methods. Nevertheless, considerable progress has been made over the last thirty years in terms of the size, shape ${ }^{7,8}$ and composition control. At present, half of the periodic table can be obtained under the NC form. The key achievements include the introduction of core-shell colloidal heterostructures, ${ }^{9}$ as this approach can not only achieve nanoparticles with a photoluminescence (PL) yield of nearly $100 \%$ but can also facilitate the maturity of the whole field. Owing to such developments, NCs have become one of the few representative nanotechnologies that have attained mass market status. In the last five years, NCs have been used as green and red downconverters for LCD displays, ${ }^{10,11}$ thereby increasing the gamut (i.e., range of color) compared to that achieved using previous technologies based on lanthanide emitters. In addition to the commercial value of this market (1 billion $\$$ per year ${ }^{12,13}$ ), this display application has demonstrated the viability of NCs to be included in industrial processes while enabling the realization of devices with a long lifetime (>10 y of operation). The switch from optically excited NCs to electrically pumped devices to reduce electrical consumption and enhance image contrast is expected to be the next challenge in this direction.

The introduction of mercury chalcogenide $(\mathrm{HgX}) \mathrm{NCs}$ was motivated by the concept of extending the optical feature of NCs into the red and near-infrared ranges, ${ }^{14}$ as shown in Figure 1 The first report of $\mathrm{HgX}$-based NCs corresponded to a CdS/HgS heterostructure. ${ }^{15} \mathrm{At}$ this stage, type I core-shell ${ }^{9,16}$ colloidal heterostructures used to enhance the PL efficiency had not yet been reported, and the method used to grow the heterostructures (i.e., the cation exchange method) was not an established synthetic method $^{17-19}$ to control the NC composition. The first targeted application of HgX NCs was their use as broadband optical amplifiers in the telecommunication range at wavelengths between 1.3 and $1.5 \mu \mathrm{m}$. Specifically, $\mathrm{HgTe} \mathrm{NCs}{ }^{20}$ were first used in the telecom domain in the early 2000s. Because NCs are continuously spectrally tunable, they offer higher flexibility for the design of IR emitting devices than their III-V counterparts obtained using epitaxy, the substrate-lattice matching for which reduces the bandgap tunability. Despite the development of $\mathrm{HgX} \mathrm{NCs,} \mathrm{as} \mathrm{reviewed} \mathrm{in} \mathrm{the} \mathrm{following} \mathrm{sections,} \mathrm{this} \mathrm{first} \mathrm{targeted} \mathrm{application}$ remains undemonstrated, although certain recent reports ${ }^{21-23}$ indicate that $\mathrm{HgX} \mathrm{NCs}$ are promising materials to obtain infrared light sources. 


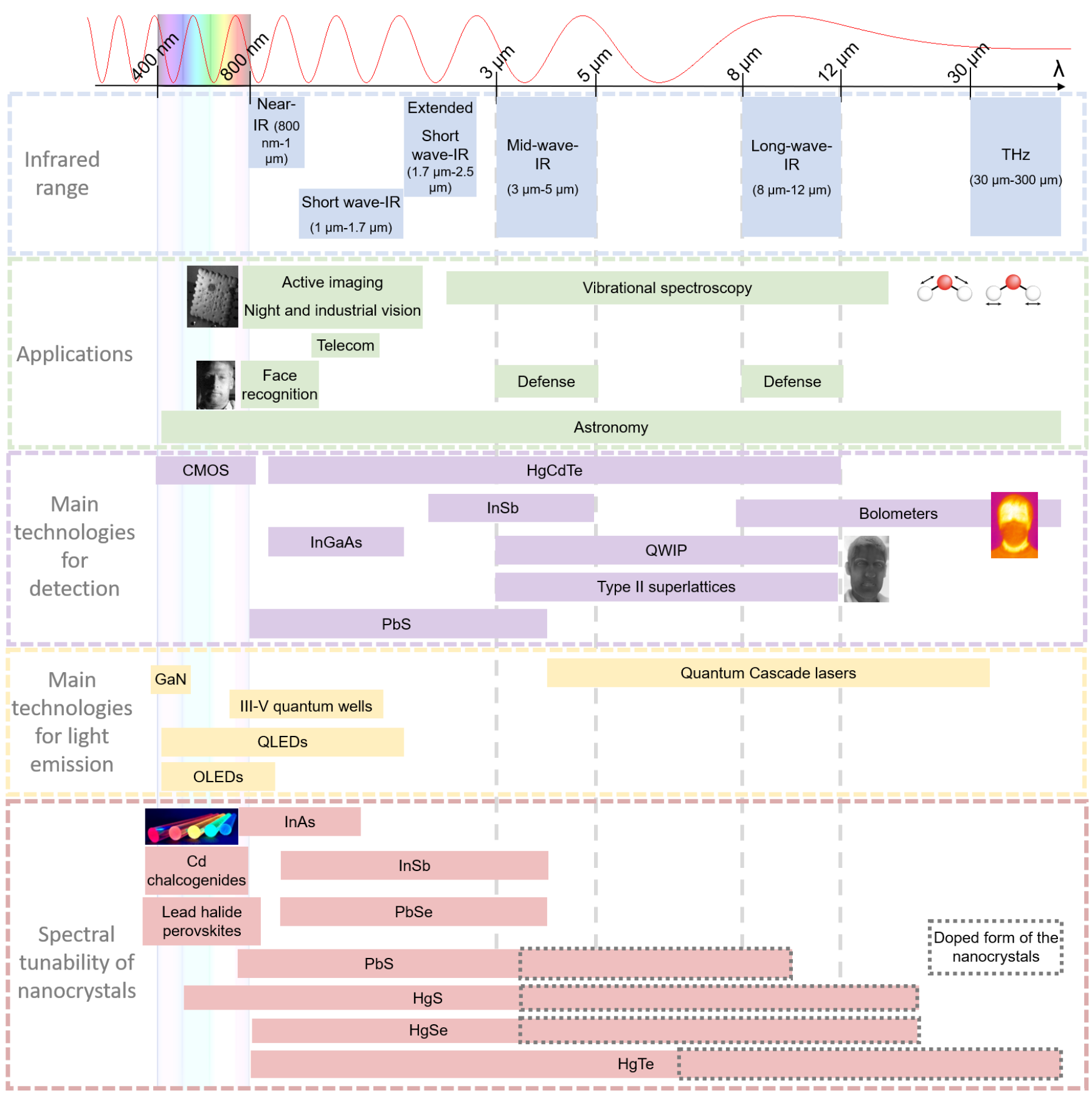

Figure 1 Electromagnetic spectrum highlighting the different parts of the infrared range. The main applications, sensor and source technologies, and tunability range for the nanocrystal optical features are also illustrated.

Moreover, considerable progress has been made in the control of the surface chemistry of NCs ${ }^{4,24-26}$ to obtain large carrier mobility films $\left(\mu \approx 1 \mathrm{~cm}^{2} \cdot \mathrm{V}^{-1} \cdot \mathrm{s}^{-1}\right)$, and the growth of $\mathrm{HgX} \mathrm{NCs}$ with larger absorption wavelengths ${ }^{3,27-30}$ has led to the emergence of HgX NCs as a viable building block for low cost IR sensing. The basic concept of using HgTe NCs instead of HgCdTe alloy is to replace the bandgap tunability from the mixed composition to quantum confinement. For HgTe, which is a semimetal, the absorption can be tuned from $1.5 \mathrm{eV}$ for the most confined form of $\mathrm{HgTe}^{31,32}$ to virtually zero. In practice, an absorption feature of as low as 20 meV has been reported. ${ }^{30}$ In a ten year time scale, the material has switched from seminal development with absorption and photoconduction in the short wave IR (SWIR) and MWIR ${ }^{1,3}$ to a versatile platform for optoelectronics. A nonexhaustive list of key developments include

- Evidence of an intraband in the steady state due to self doping $29,33,34$

- Large mobility films ${ }^{35}$ with $\mu>1 \mathrm{~cm}^{2} . \mathrm{V}^{-1} \mathrm{~s}^{-1} 36$ 
- Large absorption ${ }^{37-40}$ and emission ${ }^{21}$ from NC films due to coupling with structures to induce a light resonance

- Multicolor detectors ${ }^{41-47}$

- High detectivity devices that, for single pixels, are comparable with commercial setups ${ }^{48}$

- Focal plane arrays in the short wave ${ }^{49,50}$ and midwave infrared. ${ }^{51,52}$

This field has likely reached a transition point from being a niche topic studied by a few groups, mostly with an NC background, to becoming a field of interest for other researchers in the photonics, $\mu$-fabrication and optoelectronics fields, ${ }^{53}$ enabling the development of increasingly complex devices. The expansion of this field also extends to industry (SWIR Vision, IMEC, Qurv, Emberion are working on PbS NC-based imaging setups and QDIR, New Imaging Technologies are focusing on HgTe to expand the spectral range), leading to the emergence of a viable low cost technology for IR sensing. Such an extension of this material toward a broader community compels a review aggregating the material insights and technological developments. The scope of this review is on mercury chalcogenide (HgX: HgS, HgSe and HgTe) NCs, not on infrared NCs. In particular, the concept and performances of $\mathrm{HgX} \mathrm{NCs}$ are compared with those of PbS-based NCs. However, PbS(e) is not the focus of this review. Several reviews dedicated to $\mathrm{HgX}$ NCs have been reported. For example Green et al. focused on the synthetic aspect of NCs ${ }^{54}$, while Rogach et $a l .{ }^{55}$ considered a historical perspective in their review. The detection application is not described extensively in these documents. Several short reviews have been dedicated to HgX NCs, with a strong focus on the aspects of devices ${ }^{56,57}$ or the fundamental limits that can be achieved; ${ }^{58}$ however, none of these reviews covers all the aspects from the material growth to application while discussing the electronic structure and transport properties. It is a choice of the authors not to include biological applications of the HgX NCs. Even though certain researchers have reported on the use of HgX NCs for biolabeling in the near IR ${ }^{59}$, the presence of $\mathrm{Hg}$ has been considered detrimental for biological applications, and thus, this direction has not been extensively considered.

The present review is organized into six main chapters. The first chapter is dedicated to NC synthesis. A section is dedicated to each of the chalcogenides ( $\mathrm{S}, \mathrm{Se}$ and Te). As shape and dimensionality control is an emerging direction for $\mathrm{HgX} \mathrm{NCs,} \mathrm{a} \mathrm{section} \mathrm{is}$ dedicated to 2D nanoplatelets composed of $\mathrm{HgX}$. The subsequent section discusses the challenging growth of core-shell objects based on HgX NCs. The final part of this chapter deals with the doping ${ }^{60}$ and tunability of the carrier density. This section is of utmost importance for the discussion of optical properties (for example, the intraband absorption), transport properties (occurring at a considerably higher carrier density compared to that for other II-VI materials, to the point at which semiconductor to metal transition can occur) and device design (i.e., $p$-n junction formation).

The second chapter deals with optical properties, starting with the bulk dispersion and modifications introduced by quantum confinement. Certain results of the modelling of the electronic structure are presented: in addition to the sizing curve (i.e., energy vs. size), the multiband k.p. model, tight binding and DFT have facilitated the understanding of properties such as the fine structure of the conduction band, ${ }^{61}$ origin of the stimulated emission ${ }^{22}$ and inverted temperature dependence of the HgTe absorption spectrum. ${ }^{62}$ A subsection is focused on the optical properties of the NCs, before we discuss the absolute band alignment (vs. vacuum) of the $\mathrm{HgX} \mathrm{NCs.} \mathrm{This} \mathrm{section} \mathrm{is} \mathrm{critical} \mathrm{in} \mathrm{terms} \mathrm{of} \mathrm{two} \mathrm{key} \mathrm{aspects,} \mathrm{specifically,} \mathrm{the} \mathrm{design} \mathrm{of} \mathrm{the} \mathrm{colloidal} \mathrm{heterostructures}$ and NC-based device integration. A rational design of devices requires a deep and reliable understanding of the band alignment to move beyond empirical rules. Nevertheless, this aspect is challenging because $\mathrm{HgX} \mathrm{NCs} \mathrm{combine} \mathrm{the} \mathrm{inverted} \mathrm{band} \mathrm{ordering,}$ quantum confinement and surface chemistry dependence of their spectra, which prevents the textbook design of heterojunctions. Lastly, we dedicate a section to the carrier dynamics, wherein we discuss the specific experimental setup used to examine the relaxation of narrow bandgap semiconductors with optical features in the IR.

After defining the materials and their fundamental properties, we focus on material applications for light emission and detection. In the subsequent chapter, we review the light emission properties of the HgX NCs. We first discuss the interband and intraband photoluminescence and later describe recent progresses related to stimulated emission and electroluminescence.

Chapter 5 focuses on the transport properties of $\mathrm{HgX} \mathrm{NC}$ arrays, providing a basis for the subsequent chapter that reports on the photoconductive properties of these materials. As high carrier densities and degenerate doping are key properties of $\mathrm{HgX}$ compounds, we first discuss the influence of the carrier density on transport. Moreover, a section is dedicated to the specific surface chemistries developed for $\mathrm{HgX} \mathrm{NCs}$ to realize simultaneous increases in the carrier mobility and stability. The latter aspect is examined in depth. Finally, a section is dedicated to field effect transistors based on HgX NCs.

In the final chapter, we discuss the IR photoconduction and photodetection properties of HgX NC arrays. A section is dedicated to the specific figures of merit of IR detection, such as the spectral range, response time, responsivity, noise, signal to noise and operating temperature. We discuss the experimental determination of these figures of merit and discuss the specificities of IR NCs. The following section is dedicated to devices for IR detection based on HgX NCs as the active material. To avoid presenting an exhaustive list of devices, the devices are categorized based on their specific geometry. We start with photoconductive devices and later include certain refinements of this basic geometry, including the addition of gates for the design of phototransistors, use 
of flexible substrates, intraband detection and coupling with 2D materials. Subsequently, we review photovoltaic devices, which exhibit the highest performance at present. A subsection is dedicated to the key upgrades of multicolor sensing, devices with enhanced light-matter coupling, and devices to realize imaging (i.e., focal plane arrays).

The conclusion summarizes the main developments achieved over the past ten years, and we highlight the main directions that remain to be addressed: material optimization, enhanced understanding of the material characteristics and upgraded devices to facilitate the propagation of the technology in the market. 


\section{MATERIAL SYNTHESIS}

This review first discusses the growth of NCs to be used as building block for devices. Mercury chalcogenides are II-VI semiconductors and are thus natural candidates to extend the optical features of cadmium chalcogenide nanocrystals, which are the most investigated material in the colloidal nanocrystal form. Because mercury is located below cadmium in the periodic table, the switch from $\mathrm{Cd}$ to $\mathrm{Hg}$ is associated with an increased number of electrons and a reduced bandgap. Consequently, mercury chalcogenides are a natural candidate to address the infrared range, as discussed later. Surprisingly, the field of HgX NCs did not begin with the growth of core only nanoparticles with a spherical shape but instead through the growth of heterostructures by using a cation exchange procedure at a stage in which this procedure was not a conventionally accepted synthetic tool to grow NCs. ${ }^{15}$ This aspect led to the formation of $\mathrm{HgS} / \mathrm{CdS}$ core shell particles with absorption in the red domain. Nevertheless, it was promptly observed that core only structures offered an easier strategy to achieve such NCs. ${ }^{20}$

Even though the abovementioned materials are II-VI semiconductors, their growth differs significantly from that of Cd-based materials. In particular, colloidal synthesis of these materials is generally realized at a considerably lower temperature (typically, $100^{\circ} \mathrm{C}$ ), indicating a highly different reactivity of $\mathrm{HgX}$-based compounds compared to that of their Cd-based counterparts. Among mercury chalcogenides, ${ }^{54} \mathrm{HgTe}$ has attracted a large amount of interest, in contrast to cadmium chalcogenides, as the ease of oxidizing CdTe renders this compound more difficult to grow and study than CdSe or CdS. The vicinity of HgTe to the HgCdTe alloy, which is already used for IR detection, has facilitated the development and understanding of this compound. Colloidal growth of HgTe has attained a high level of maturity with unique size tunability (from few $\mathrm{nm}$ to $\mu \mathrm{m}$ ), ${ }^{30}$ high monodispersity and shape control. $^{31,63,64}$ The two other chalcogenides, HgS and HgSe, have been less investigated; however, the recent emergence of intraband transition in this material may change this trend. ${ }^{65,66}$ Among the $\mathrm{HgX}$ compounds, $\mathrm{HgS}$ exhibits two specificities. First, the bulk material presents a gap, while HgSe and HgTe are semimetals in the bulk form. In addition, HgS exists in two crystalline forms: zinc blende and cinnabar. The latter form is promising to obtain chiral optical features.

This chapter is organized as follow: we first review the growth of core only objects for the three chalcogenides. Then, we discuss shape control relative to the growth of $\mathrm{HgX} \mathrm{NCs}$ with a focus on the growth of 2D nanoparticles (nanoplatelets ${ }^{67}$ ). A section is dedicated to $\mathrm{HgX}$-based heterostructures. In addition to the traditional enhancement of the photoluminescence efficiency, the growth of the heterostructure is a key step to obtain air stable and temperature resistant NCs. Finally, we discuss the doping of $\mathrm{HgX} \mathrm{NCs,} \mathrm{which} \mathrm{is} \mathrm{a} \mathrm{central} \mathrm{aspect} \mathrm{for} \mathrm{device} \mathrm{integration.} \mathrm{In} \mathrm{this} \mathrm{section,} \mathrm{we} \mathrm{review} \mathrm{not} \mathrm{only} \mathrm{the} \mathrm{inherent} \mathrm{doping} \mathrm{after} \mathrm{synthesis}$ but also the strategies use to tune the doping magnitude through physicochemical methods.

\subsection{HgTe nanocrystal synthesis}

Among the three chalcogenides (HgS, $\mathrm{HgSe}$ and $\mathrm{HgTe}$ ), HgTe is the most studied from the synthesis and application viewpoints, which may seem paradoxical from the material growth perspective since Te-based chalcogenides are less available than their $S$ and Se counterparts. In addition, Te-based semiconductors usually present a lower work function, ${ }^{68}$ making them more prone to oxidation. HgTe in its bulk form has been well developed for infrared detection for over fifty years. ${ }^{69}$ This application has encouraged the development of growth methods and has facilitated the understanding of the HgTe electronic structure. ${ }^{70-72}$ In particular, HgCdTe alloys exhibit broad tunability of the absorption spectrum from the near IR to longwave IR. Photocarriers exhibit a large lifetime of up to $1 \mu \mathrm{s}$, which is favorable for the charge collection in IR sensors, and the dark current in this material is among the lowest for IR detectors, ${ }^{73,74}$ enabling higher operating temperatures. Although this material is toxic, until now, the performances recorded with HgTe-based materials are higher than those of any other material. This aspect is more notable for NCs dedicated to IR. HgTe exhibits the highest spectral tunability (from below 1 to above $100 \mu \mathrm{m}$ ), as shown in Figure 2a. In comparison, quantum confinement allows the tuning of the CdSe band edge from only $400 \mathrm{~nm}$ to $700 \mathrm{~nm}$, while doping NCs in ITO (tin doped indium oxide) only shifts the absorption from $2 \mu \mathrm{m}$ to $9 \mu \mathrm{m} .{ }^{75,76}$ The tunability in both cases is considerably small (less than the two orders of magnitude) compared to that achieved by HgTe. 

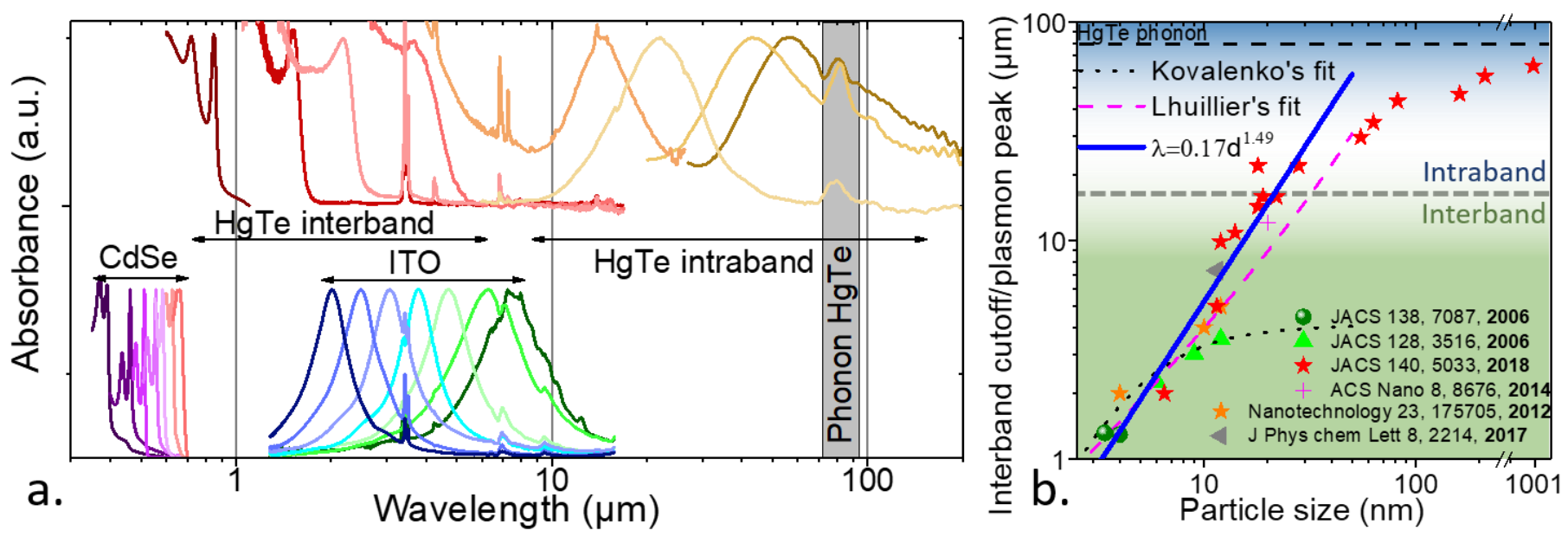

Figure 2 a. Absorption spectra for various sizes of $\mathrm{HgTe}$ and $\mathrm{CdSe}$ and various doping values of ITO NCs. b. Wavelength of the lowest energy absorption feature as a function of the particle size for $\mathrm{HgTe}$ NCs, obtained from various references.

Until now, the use of less toxic compounds in IR applications has led to a dramatically reduced performance. ${ }^{76-79}$ As a bulk material, InSb exhibits a similar performance as that of $\mathrm{HgCdTe}$ in the 3-5 $\mu \mathrm{m}$ range; however, the NC counterpart is not as widely investigated as HgTe. InSb NCs are promising materials ${ }^{80-82}$; however, their corresponding integration in devices remains unexplored. Lead chalcogenides are the most notable competitors to HgTe. ${ }^{49}$ Among these materials, PbS is the most developed, exhibiting a high synthesis maturity, ${ }^{83,84}$ tunable absorption in the near IR, mature transport, ${ }^{85}$ and device integration. ${ }^{86-88}$ However, the material becomes challenging to manage above $2 \mu \mathrm{m}^{89}$ and is limited by its bulk bandgap ( $\left.\approx 4 \mu \mathrm{m}\right) .{ }^{90}$ Owing to these considerations, HgTe NCs represent a highly versatile platform for IR photonics and optoelectronics. Initially (2000-2010), the interest in HgTe NCs was not driven by IR detection as for the bulk case but rather by light amplification. ${ }^{55}$ Only later (post 2006) did HgTe NCs emerge as a promising low cost alternative ${ }^{3,91}$ to bulk HgCdTe alloys.

Although HgX NCs are II-VI semiconductors, as is CdSe, their synthesis involves certain specificities. For example, the synthesis temperature is significantly reduced compared to that of CdSe NCs, as indicated in Table 1 . The synthesis strategies used to obtain HgTe NCs can be categorized as the aqueous method, which has been extended to polar solvents, and the organic medium method.

Table 1 Conditions for the selection of relevant syntheses for HgTe NCs.

\begin{tabular}{|c|c|c|c|c|c|c|c|}
\hline $\begin{array}{l}\mathrm{Hg} \\
\text { precursor }\end{array}$ & Te precursor & Ligand & $\begin{array}{l}\text { Solvent / } \\
\text { Temperature }\end{array}$ & Method & $\begin{array}{l}\text { Size } \\
(\mathrm{nm})\end{array}$ & $\begin{array}{l}\text { Optical } \\
\text { prop }\end{array}$ & Year/Ref \\
\hline $\mathrm{Hg}\left(\mathrm{ClO}_{4}\right)_{2}$ & $\mathrm{H}_{2} \mathrm{Te}$ & thioglycerol & $\begin{array}{l}\text { Water/ } \\
\text { RT }\end{array}$ & & $3-6$ & $\begin{array}{l}\text { Up to } \\
1.4 \mu \mathrm{m}\end{array}$ & $1999 / 20$ \\
\hline $\mathrm{Hg}\left(\mathrm{ClO}_{4}\right)_{2}$ & $\mathrm{H}_{2} \mathrm{Te}$ & $\begin{array}{l}\text { thioglycerol, } \\
\text { thioglycolic acid, I- } \\
\text { cysteine, } \\
\text { mercaptoethanol, } \\
\text { and } \\
\text { mercaptoethylamine }\end{array}$ & $\begin{array}{l}\text { Water/ } \\
\text { RT and then } \\
\text { heating at } 80 \\
{ }^{\circ} \mathrm{C}\end{array}$ & & $3-12$ & $\begin{array}{l}\text { Up to } \\
3.7 \mu \mathrm{m}\end{array}$ & $2006 / /^{27}$ \\
\hline $\mathrm{HgCl}_{2}$ & $\mathrm{H}_{2} \mathrm{Te}$ & $\begin{array}{l}\text { Triethylamine, } \\
\text { tripropylamine - } \\
\text { furanmethanethiol }\end{array}$ & $\begin{array}{l}\text { DMSO or DMF } \\
\text { and } \\
\text { diphenylether/ } \\
40-80^{\circ} \mathrm{C}\end{array}$ & & $4-10$ & $\begin{array}{l}\text { Up to } \\
4.5 \mu \mathrm{m}\end{array}$ & $2020 / 92$ \\
\hline $\mathrm{HgBr}_{2}$ & TOP:Te & TOPO & $\begin{array}{l}\text { TOPO/ } \\
70-100{ }^{\circ} \mathrm{C}\end{array}$ & $\begin{array}{l}\text { Hot } \\
\text { injection }\end{array}$ & $3-17$ & $\begin{array}{l}\text { Above } \\
1.7 \mu \mathrm{m}\end{array}$ & $2013 / /^{93}$ \\
\hline $\mathrm{Hg}(\mathrm{OAc})_{2}$ & TOP:Te & ethanol & $\begin{array}{l}\text { Ethanol/ } \\
-78^{\circ} \mathrm{C}\end{array}$ & $\begin{array}{l}\text { Low }{ }^{\mathrm{T}} \\
\text { injection }\end{array}$ & $3-4$ & $\begin{array}{l}\text { Up to } \\
1.6 \mu \mathrm{m}\end{array}$ & $2006 / /^{94}$ \\
\hline
\end{tabular}




\begin{tabular}{|c|c|c|c|c|c|c|c|}
\hline $\mathrm{Hg}(\mathrm{OAc})_{2}$ & $\mathrm{Te}$ & pyridine & $\begin{array}{l}\text { Butanol/ } \\
0-90^{\circ} \mathrm{C}\end{array}$ & $\begin{array}{l}\text { Injection } \\
\text { of cation } \\
\text { on anion }\end{array}$ & $5-12$ & $\begin{array}{l}\text { Up to } 5 \\
\mu \mathrm{m}\end{array}$ & $2011 /^{3}$ \\
\hline $\mathrm{HgCl}_{2}$ & TOP:Te & DDT & $\begin{array}{l}\text { OLA/ } \\
60-120^{\circ} \mathrm{C}\end{array}$ & & $5-12$ & $\begin{array}{l}\text { up to } 5 \\
\mu \mathrm{m}\end{array}$ & $2011 / 95$ \\
\hline $\mathrm{HgCl}_{2}$ & $\mathrm{TMS}_{2} \mathrm{Te}$ & DDT & OLA & & $5-12$ & $\begin{array}{l}\text { Up to } 5 \\
\mu \mathrm{m}\end{array}$ & $2017 / 63$ \\
\hline $\begin{array}{l}\mathrm{HgX}_{2} \\
(\mathrm{X}=\mathrm{Cl}, \mathrm{Br}, \mathrm{I})\end{array}$ & TOP:Te & DDT & $\begin{array}{l}\text { OLA/ } \\
60-300{ }^{\circ} \mathrm{C}\end{array}$ & $\begin{array}{l}\text { Injection } \\
\text { of both } \\
\text { cations } \\
\text { and } \\
\text { anions in } \\
\text { a hot } \\
\text { solvent }\end{array}$ & $\begin{array}{l}5- \\
1000\end{array}$ & $\begin{array}{l}2-60 \\
\mu \mathrm{m}\end{array}$ & $2018 / 30$ \\
\hline Liquid $\mathrm{Hg}$ & TOPTe & DDT & $\begin{array}{l}\text { OLA/ } \\
60-150{ }^{\circ} \mathrm{C}\end{array}$ & $\begin{array}{l}\text { In situ } \\
\text { formation } \\
\text { of } \quad \mathrm{Hg} \\
\text { thiolate }\end{array}$ & $3-20$ & $\begin{array}{l}1.3-15 \\
\mu \mathrm{m}\end{array}$ & $2020 / 96$ \\
\hline
\end{tabular}

\subsubsection{Synthesis in a polar solvent}

Incredibly early reports on HgTe NCs and their alloying with $\mathrm{HgCdTe}$ can be found. The early concepts were likely motivated by the realization of polycrystalline films by using chemical bath deposition. By mixing mercury halides with sodium telluride in methanol, large ( 1 to $100 \mathrm{~nm}$ ) nanoparticles of $\mathrm{HgTe}$ and $\mathrm{HgCdTe}$ were obtained by Müllenborn et al. ${ }^{97}$ This research was followed by a period of low interest in $\mathrm{HgX} \mathrm{NCs,} \mathrm{likely} \mathrm{corresponding} \mathrm{to} \mathrm{the} \mathrm{period} \mathrm{in} \mathrm{which} \mathrm{CdSe} \mathrm{NCs} \mathrm{became} \mathrm{popular.} \mathrm{Rogach} \mathrm{et} \mathrm{al,} \mathrm{inspired}$ by their earlier work on CdTe NCs, ${ }^{98,99}$ developed a water-based synthesis approach for HgTe NCs. ${ }^{20,100}$ Mercury perchlorate was dissolved in water in the presence of thioglycerol as a ligand, and $\mathrm{H}_{2} \mathrm{Te}$ was bubbled in this solution, acting as a Te $\mathrm{Te}^{2-}$ source. The reaction could be more effectively performed under alkaline conditions ( $\mathrm{pH}=9-12$, typically). The obtained HgTe NCs exhibited a tunable size from 3 to $6 \mathrm{~nm}$, as shown in Figure 3a and Figure 26 a in terms of transmission electron microscopy (TEM) images and optical spectra, respectively. Synthesis was conducted at room temperature, and the NC emission reached the telecom range. Later, Kovalenko et al. introduced an additional Ostwald ripening step by heating the solution after growth at $80{ }^{\circ} \mathrm{C} .{ }^{27}$ Larger particles, 10-12 nm (Figure $3 \mathrm{~b}-\mathrm{c}$ ), were obtained, and the PL signal reached $3.7 \mu \mathrm{m}$, as shown in Figure 26b. More recently, Rogach and coworkers expanded the concept of this synthesis to nonwater-based polar solvents by performing syntheses in DMSO ${ }^{101}$ and $\mathrm{DMF}^{92}$. Using this approach, the PL spectra were pushed to $4.5 \mu \mathrm{m}$. This approach is also relevant for IR detection since NCs are readily available in polar solvents in which the particles are capped by short ligands compatible with transport. This approach has already been utilized for PbS nanocrystals by realizing NC formation without long insulating ligands to obtain an ink that can be spincoated to form a conductive film of NC with a low density of the surface traps. ${ }^{102}$
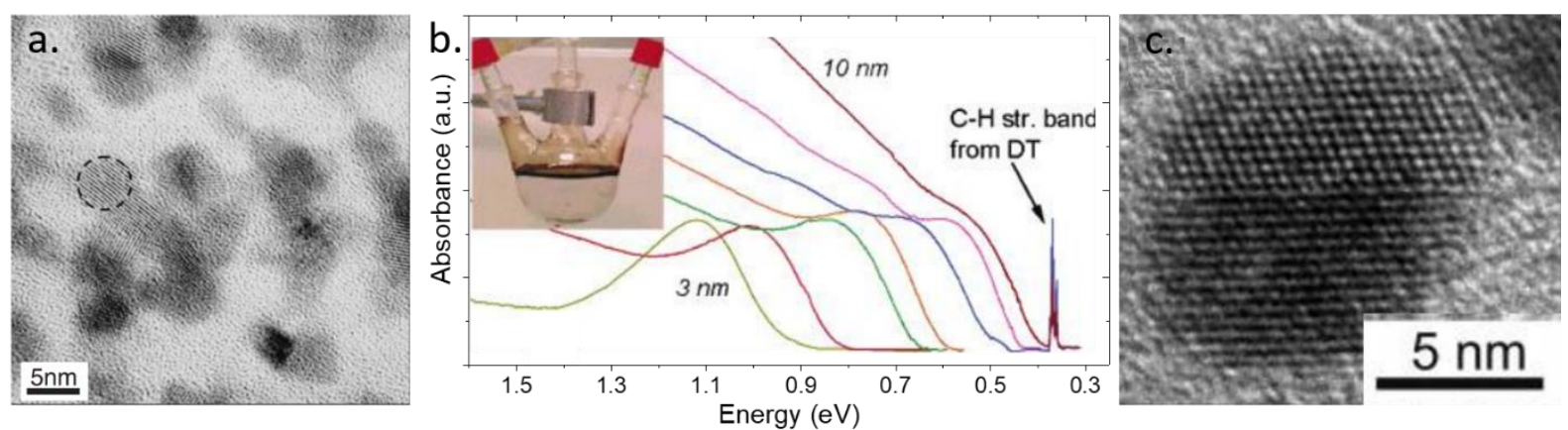

Figure 3 a. High resolution TEM image of HgTe NCs obtained through the synthesis described in ref 20. Part $a$ is adapted from ref ${ }^{103}$ - Copyright (2005) The Japan Society of Applied Physics. $b$. Representative room temperature absorption spectra of DDT-capped $\mathrm{HgTe} \mathrm{NCs} \mathrm{in} \mathrm{CCl}_{4}$. C. TEM image of $\approx 9 \mathrm{~nm} D D T$-capped HgTe NCs emitting at $3 \mu \mathrm{m}$. Parts $b$ and $c$ are adapted with permission fro $m$ ref 27 - Copyright (2006) American Chemical Society. 


\subsubsection{Synthesis in an organic medium}

A key limitation of aqueous syntheses is the use of $\mathrm{H}_{2} \mathrm{Te}$, which makes the synthetic setup considerably more complex than that for solid/liquid based syntheses. Alternative growth methods, typically conducted in organic media, have been developed. As for water-based syntheses, early evidence for mercury chalcogenides NC organic syntheses can be found. In the pioneering work by Murray, mostly dedicated to CdSe, mercury chalcogenides are also mentioned. ${ }^{6}$ In the earliest stage of the hot injection method, highly reactive precursors are used, such as metal alkyl. Diethyl cadmium is highly reactive and easily forms pyrophoric compounds; however, the toxicity of its mercury counterparts is even higher. ${ }^{104}$ Consequently, additional approaches for synthesizing HgTe NCs based on alkyl mercury have not been proposed. Nevertheless, the use of phosphine as a capping ligand has been retained. Green et al. proposed a mild temperature $\left(70^{\circ} \mathrm{C}\right)$ synthesis in which a mercury halide reacts with the Te complex and trioctylphosphine (TOP:Te) in a mixture of a coordinating solvent (TOPO and long chain amines). ${ }^{93,105}$ This combination of the $\mathrm{Hg}^{2+}$ salt and TOP:Te in the presence of an amine has been the backbone of most syntheses approaches developed subsequently. The authors reported fairly large NCs (up to $17 \mathrm{~nm}$ ), as shown in Figures $4 a-b$, with a featureless optical spectrum. Although the lack of excitonic features might correspond to polydispersity, such features likely appear at wavelengths larger than the reported data. Piepenbrock et al. enhanced this method to realize a lower polydisperse synthesis of small NCs (3-4 nm), as shown in Figures $4 \mathrm{~d}$. This synthesis was based on the use of mercury acetate as the $\mathrm{Hg}$ precursor in the presence of long chain amines and ethanol as the solvent. The TOP:Te was slowly added while cooling the reaction to $-78{ }^{\circ} \mathrm{C}$ (dry ice). The NCs exhibited an absorption shoulder at $1200-1600 \mathrm{~nm}$, as shown in Figures 4c.

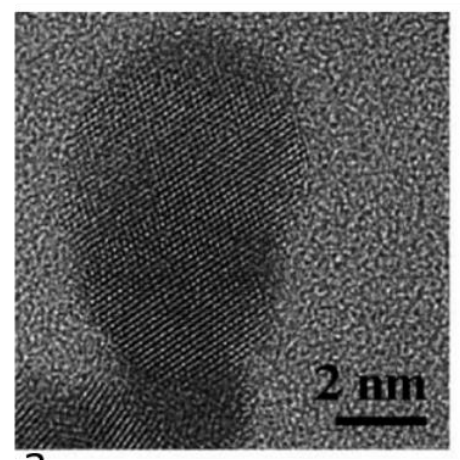

a.

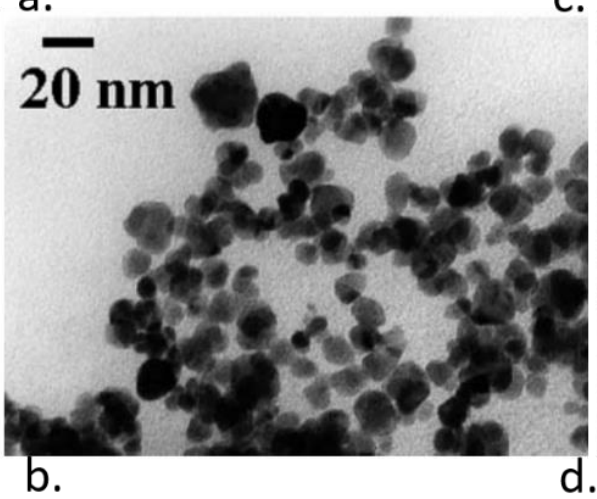

c.
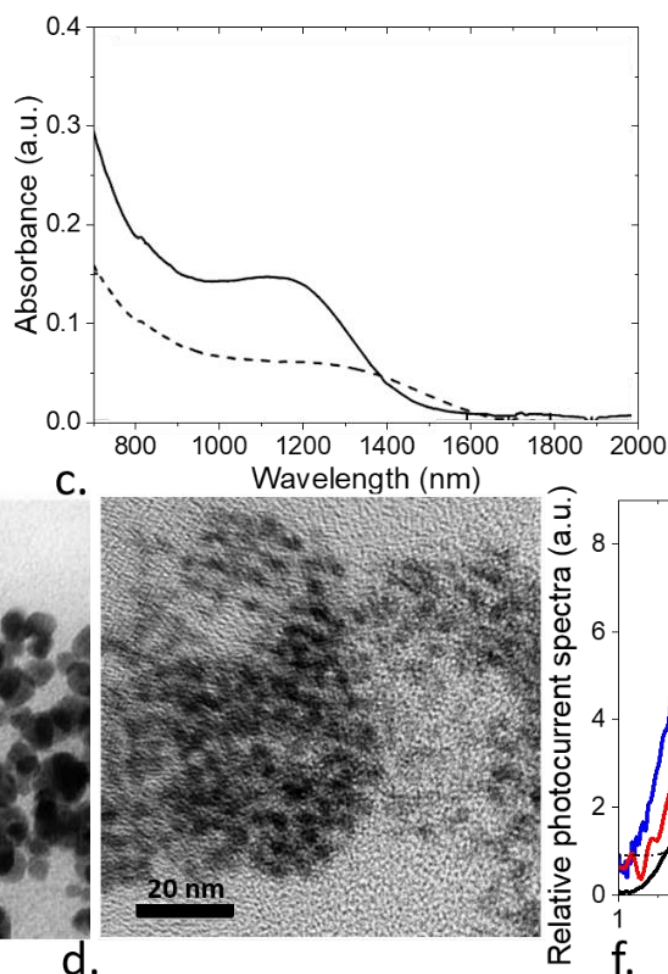
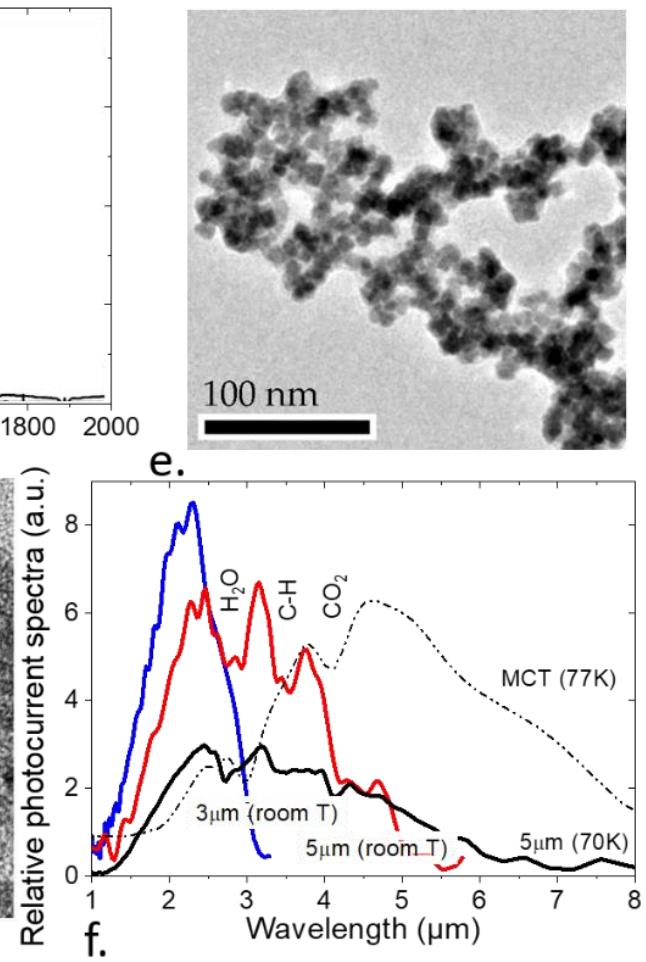

e.

Figures 4 a. and b. TEM images of HgTe capped with octadecylamine and TOPO prepared at $70^{\circ} \mathrm{C}$. Parts $a$ and $b$ are used with permission of the Royal Society of Chemistry, from ref ${ }^{93}$ - permission conveyed through Copyright Clearance Center, Inc. c. Absorption spectra of HgTe nanoparticles. $d$. TEM image of HgTe QDs redissolved in toluene. Parts $c$ and d are adapted with permission from ref ${ }^{94}$ - Copyright (2006) American Chemical Society. e. TEM image of HgTe CQDs with an absorption onset at $5 \mu \mathrm{m}$. $\mathrm{f}$. Fourier transforms of the interferograms measured from films of $\mathrm{HgTe} \mathrm{NCs}$ with cutoff wavelengths at 3 and $5 \mu \mathrm{m}$. Parts $e$ and $f$ are adapted from ref 3.

In 2011, Keuleyan et al. introduced the first synthesis technique to realize absorption and photoconduction up to $5 \mu \mathrm{m}$, as shown in Figures $4 f^{3}$ This approach represented a breakthrough from an application point of view because the material was sufficiently red to enable thermal imaging with NCs. However, the associated quality was low, with highly severe aggregation, as indicated in the TEM image shown in Figures 4e. In addition, this synthesis was relatively different from the existing approaches. First, this approach was the only one based on elemental Te. While most syntheses rely on hot injection of the Te $\mathrm{e}^{2-}$ anion, this procedure relies on the introduction of Te powder, pyridine, and butanol to the flask and injection of the cation salt (mercury acetate). Although the inherent aggregation of this synthesis technique was appealing for transport to achieve a high carrier mobility, the 
wide size dispersion of the NCs led to extremely broad optical features with only the tail of absorption actually reaching the 3-5 $\mu \mathrm{m}$ range. The alternative synthesis led to larger particles (up to $12 \mathrm{~nm}$ ); however, the monodispersity was required to be enhanced.

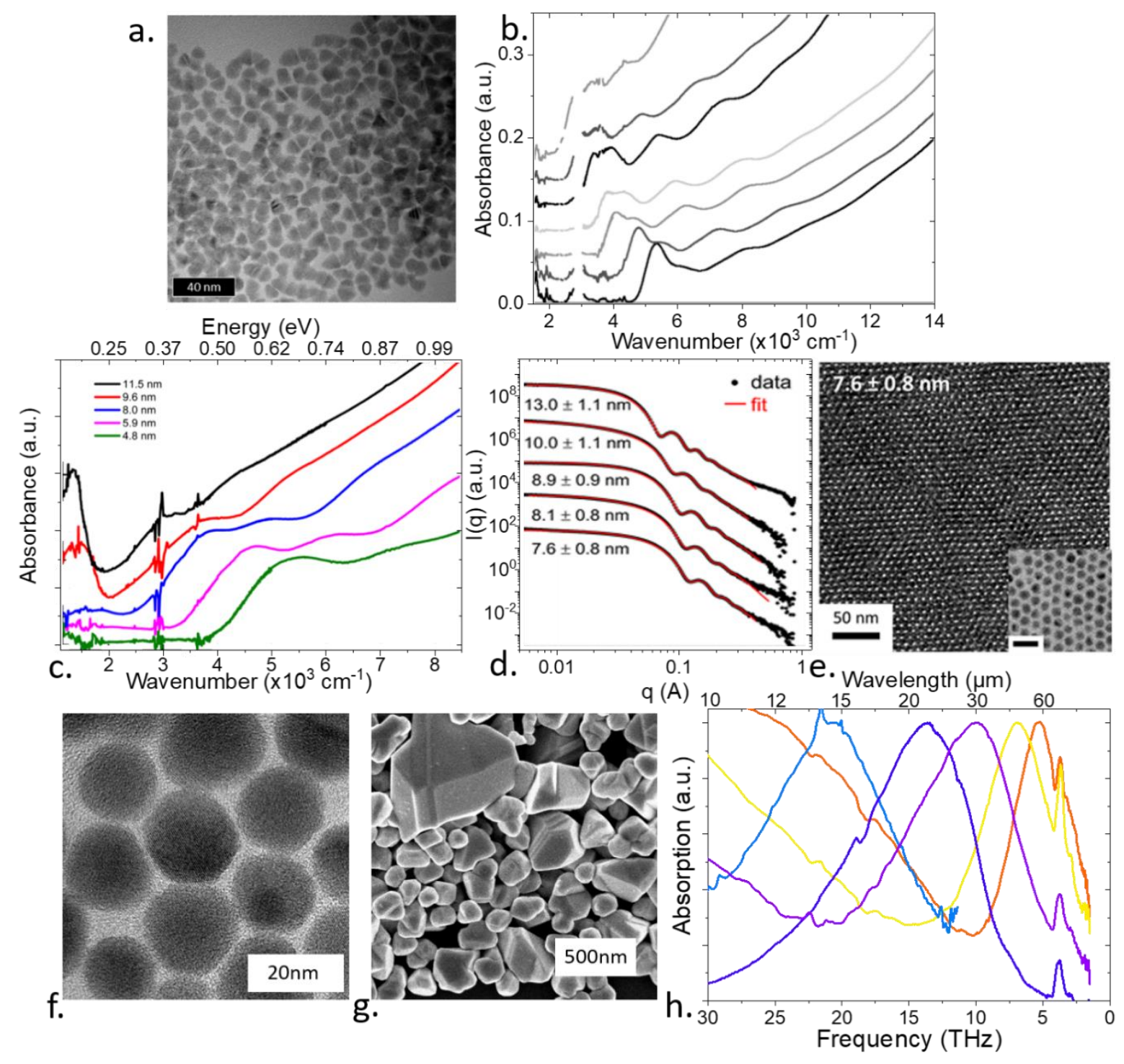

Figure 5 a. TEM image of $9.1 \mathrm{~nm}$ HgTe NCs. b. Absorption spectra of differently sized HgTe NCs showing multiple features. Parts $a$ and $b$ are adapted with permission from ref ${ }^{28}$ - Copyright (2014) American Chemical Society. c. Absorption spectra of the HgTe CQD solution showing intraband absorption for the largest particles. Part $c$ is adapted with permission from ref ${ }^{63}$ - Copyright (2018) American Chemical Society. $d$. Small angle $X$-ray scattering signal and the corresponding fits for colloidal solutions of $\mathrm{HgTe}$ NCs exhibiting a reasonable size control ( $\approx 10 \%$ distribution) across a range of samples. e. TEM image of a HgTe NC-based superlattice upon slow evaporation of the solvent. The inset image shows a monolayer of the same particles dropcast from a dilute solution (scale bar, 10 $\mathrm{nm})$. Parts $d$ and e are adapted with permission from ref ${ }^{61}$ - Copyright (2019) American Chemical Society. $f$ and $g$ TEM images of large HgTe NCs with absorption above $10 \mu \mathrm{m}$. $h$. Series of infrared spectra for extremely large HgTe NCs. Parts $f-h$ are adapted with permission from ref ${ }^{30}$ - Copyright (2018) American Chemical Society.

Around 2010, a series of conceptually similar procedures were demonstrated for the growth of HgTe NCs. ${ }^{22,95,106,107}$ Most of the concepts were based on the use of the conventional hot injection method (injection of an anion in a cation solution at a "high" temperature). $\mathrm{A} \mathrm{Hg}^{2+}$ salt from $\mathrm{HgCl}_{2}$ or mercury acetate was dissolved in presence of an amine, a thiol, and possibly oleic acid. ${ }^{107}$ A noncoordinating solvent could also be added. ${ }^{106}$ The Te precursor was obtained from Te complexed with phosphine, as initially proposed by Green et al. ${ }^{93}$ This approach is widely used for photoconduction devices. ${ }^{35,48,108}$ Thus, it is worth describing the role of each component. The reaction is conducted under mild temperatures (60 to $120^{\circ} \mathrm{C}$ ): a higher temperature corresponds to 
larger particles. Similarly, the reaction duration can be considered as a size control parameter. At a temperature of $60{ }^{\circ} \mathrm{C}$ and duration of $3 \mathrm{~min}$, particles with a band edge at $\approx 6000 \mathrm{~cm}^{-1}(\approx 720 \mathrm{meV}$ or $1.8 \mu \mathrm{m})$ can be obtained, corresponding to a particle size of approximately $6 \mathrm{~nm}$; the conditions of $120^{\circ} \mathrm{C}$ and 15 min yield a band edge at $2000 \mathrm{~cm}^{-1}(\approx 250$ meV or $5 \mu \mathrm{m})$, leading to a particle size of approximately $12 \mathrm{~nm}$, as shown in Figure 5a. To obtain even larger particles, a regrowth step in which the Te precursor is injected dropwise must be performed. ${ }^{28} \mathrm{The} \mathrm{Hg}$ to Te ratio is generally set as approximately 1 . An amine must be used to realize monodisperse syntheses, ${ }^{109,110}$ with a more dilute synthesis corresponding to a lower polydispersity. ${ }^{28}$ Under the dilute condition, an NC with an absorption spectra involving up to six clearly defined optical transitions can be obtained, ${ }^{28,30}$ as shown in Figure 5b. In this case, the HgTe NCs in the IR range are as desirable as the Cd-based NCs in the visible range. The introduction of carboxylic acid (even using mercury oleate as the precursor ${ }^{107}$ ) and thiol during the synthesis accelerates growth. Because the synthesis is conducted at mild temperatures, lowering the temperature to RT is not sufficient to stop growth. Thus, a long chain thiol (dodecanethiol) is generally introduced to render the NC surface inaccessible to the remaining precursors. Goubet et al. used the same approach; however, both the $\mathrm{Hg}$ and Te precursors were injected into the hot solvent. ${ }^{30}$, as earlier proposed to obtain highly monodisperse PbSe NCs. ${ }^{111}$ In this case, the motivation was to avoid degradation of the Hg precursor. Using this strategy, the authors succeeded in realizing the growth of $\mathrm{HgTe}$ NCs from $60-300{ }^{\circ} \mathrm{C}$ and obtained an extremely broad size tunability from 5 to $1000 \mathrm{~nm}$, as shown in Figure $5 \mathrm{f}-\mathrm{g} .{ }^{30}$ Using such large particles, the authors reported absorption up to the $60 \mu \mathrm{m}$ peak and up to $200 \mu \mathrm{m}$ for the cutoff, ${ }^{30}$ as shown in Figure $5 \mathrm{~h}$. These NCs were the first reported structures larger than the HgTe Bohr radius ${ }^{112}$ $(40 \mathrm{~nm})$ to present absorption in the $\mathrm{THz}$ range.

An interesting evolution of this method was the replacement of the phosphine-based Te precursor with bis(trimethylsilyl)telluride $\left(\mathrm{TMS}_{2} \mathrm{Te}\right){ }^{63}$ Bis(trimethylsilyl)sulfide has been widely used to realize the growth of CdS shells and synthesize PbS cores. ${ }^{83}$ $\mathrm{Bis}$ (trimethylsilyl)chalcogenides are highly reactive forms of chalcogenides that rapidly decompose and behave as reduced forms of anions $\left(\mathrm{S}^{2-}, \mathrm{Se}^{2-}\right.$, and $\left.\mathrm{Te}^{2-}\right)$. When $\mathrm{TMS}_{2} \mathrm{Te}$ is used to grow $\mathrm{HgTe}$, more spherical shapes can be achieved; in contrast, the reaction based on TOP:Te leads to branched or faceted shapes. These spherical particles enable the formation of HgTe NC-based superlattices ${ }^{61}$ (see Figure $5 \mathrm{~d}-\mathrm{e}$ ) and can be used to enhance the carrier mobility by allowing a higher packing density in the film. ${ }^{36}$ However, this strategy is difficult to generalize owing to the high cost and small lifetime of TMS 2 Te. Nevertheless, this synthesis is essential since it is the first reported intraband absorption for HgTe NCs, as described in the section dedicated to doping and Figure $5 c$.

Recently, Goubet et al. ${ }^{96}$ addressed the scale-up of the synthesis. Current syntheses are significantly diluted $\left(<10\right.$ g. $\mathrm{L}^{-1}$ of NCs per volume of solution, as shown in Figure 6e). This issue is critical to scalability, as high dilution conditions require greater effort to obtain the same amount of NCs. Specifically, the setup and precursors are relatively inexpensive, and human effort is the main cost related to NC growth. ${ }^{113}$ In addition, a more concentrated solution generally reduces the amount of solvent (and associated cost) required in the NC cleaning steps. Therefore, it is necessary to develop more concentrated syntheses. As mentioned previously, most strategies to grow $\mathrm{HgTe} \mathrm{NCs}$ rely on the use of $\mathrm{Hg}^{2+}$ salt. A key issue with the existing syntheses is the low solubility of $\mathrm{Hg}$ halide in organic solvents, especially at the temperatures used for $\mathrm{HgTe} \mathrm{NC}$ growth (i.e., below $100{ }^{\circ} \mathrm{C}$ ). This aspect dramatically reduces the synthesis concentration. The use of an aprotic polar solvent (DMF, DMSO) can help increase the salt solubility and achieve a nanocrystal concentration of up to $60 \mathrm{~g} . \mathrm{L}^{-1} .^{92}$ Alternatively, Goubet et al. proposed the use of Hg thiolate as a strong soluble form of $\mathrm{Hg}$ in oleylamine (OLA) and demonstrated that $\mathrm{Hg}$ thiolate can be formed from liquid $\mathrm{Hg}$ sonicated with DDT, ${ }^{96}$ as shown in Figure 6a and b. This mixture reacts with TOP:Te, as shown in Figure 6c. Using this approach, the authors demonstrated the synthesis of HgTe NCs from NIR to longwave IR, as shown in Figure $6 \mathrm{~d}$. Up to $7 \mathrm{~g}$ of $2 \mu \mathrm{m}$ cutoff HgTe NCs could be achieved from $150 \mathrm{~mL}$ of liquid (including the reaction and cleaning solvents), ${ }^{96}$ as shown in Figure $6 \mathrm{e}$. 


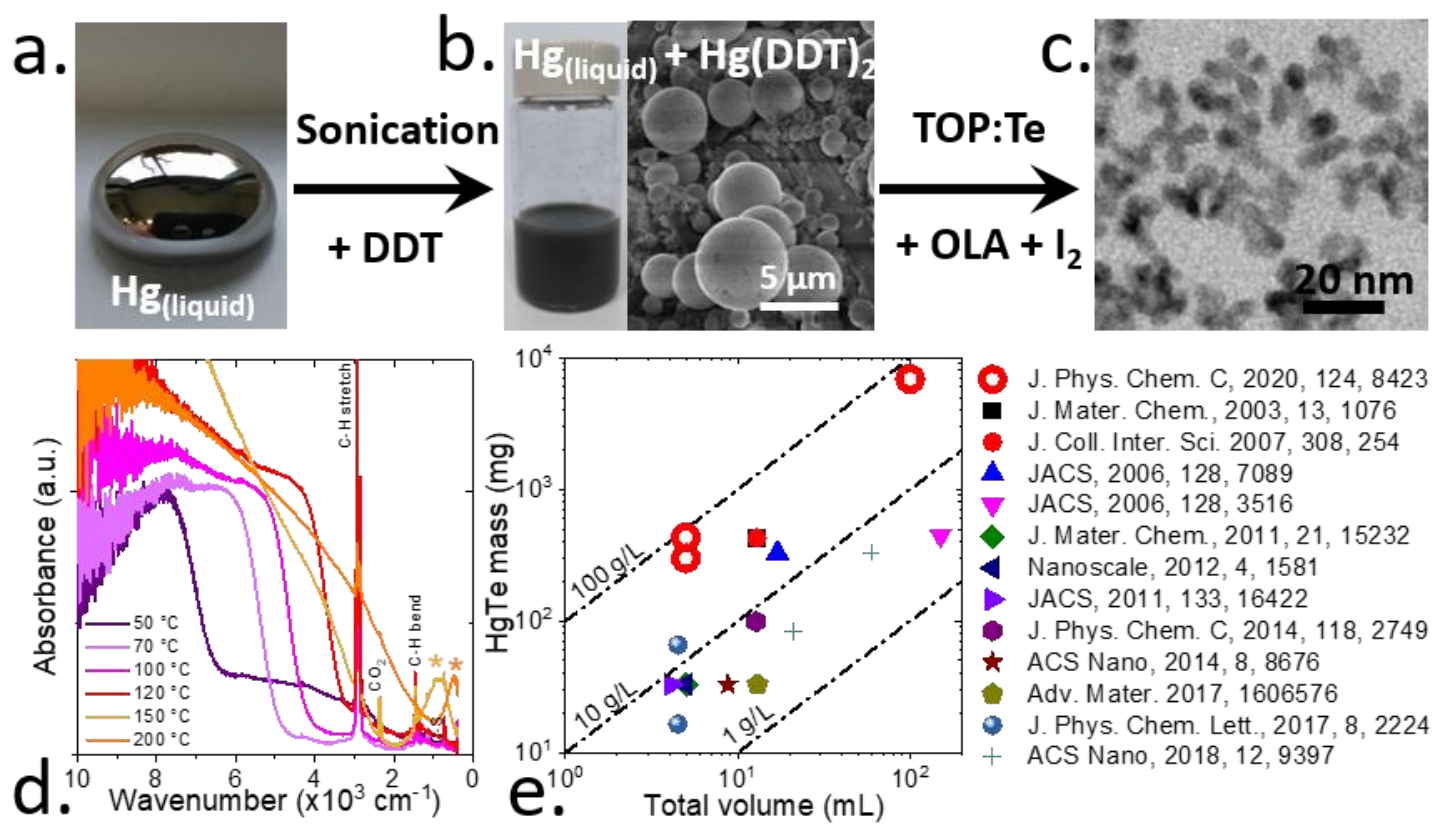

Figure 6 a. Image of a liquid $\mathrm{Hg}$ drop. b. Optical and SEM images of a solution of mercury dodecylthiolate prepared from liquid $\mathrm{Hg}$. C. TEM picture of $\mathrm{HgTe} \mathrm{QD}$ s synthesized using liquid $\mathrm{Hg}$ as a precursor. d. Absorbance spectra of $\mathrm{HgTe} Q \mathrm{QDs}$ synthesized from 50 to $200{ }^{\circ} \mathrm{C}$ by using liquid $\mathrm{Hg}$ as the precursor. e. Comparison of the mass yield obtained in this work to those reported previously, assuming the total conversion of the limiting reagent into a $\mathrm{HgTe}$ material. The figures are adapted with permission from ref ${ }^{96}$ - Copyright (2018) American Chemical Society.

Before completing this discussion related to material growth, the sizing curve must be mentioned as a valuable tool to empirically correlate the particle size to the absorption feature. For HgTe, two models have been proposed. The first model was reported by Kovalenko et al. ${ }^{27}$, which provided the energy of the interband transition (in eV) as a function of the particle (d in $\mathrm{nm}$ ) size: $E=0.3+0.2 d^{-1}+5.7 d^{-2}$. The other model was proposed by Lhuillier et al., which was obtained by mixing a two-band k.p model through empirical fitting. ${ }^{114}$ In this case, the band edge wavelength (in $\mu \mathrm{m}$ ) was related to the particle radius (R) according to the following expression: $\lambda=\frac{30.5}{\sqrt{1+\left(\frac{43}{R}\right)^{2}}-1}$. Nevertheless, these two expressions are old, and the size range that can be obtained has been considerably extended, as shown in Figure $2 \mathrm{~b}$. When considering only the interband transition (i.e., excluding the feature resulting from the intraband and plasmonic absorption observed in the largest NCs), an empirical expression to fit the wavelength vs. size (i.e., effective diameter in $\mathrm{nm}$ ) is $\lambda=0.17 d^{1.49}$, as shown in Figure $2 \mathrm{~b}$.

\subsection{HgSe nanocrystal synthesis}

In addition to telecom motivations, ${ }^{115}$ similar to those reported for HgTe, the initial motivation for HgSe NCs was to push the optical feature of CdSe toward larger wavelengths to attain an infrared transparency window in which bioimaging could be realized. ${ }^{116}$ Most of the initial papers on $\mathrm{HgS}(\mathrm{e})$ reported on its optical features in the red ${ }^{116}$ and near-infrared ranges ${ }^{115}$ as well as the UV range. ${ }^{117}$ The latter observation may appear surprising since the bulk bandgap of HgSe is smaller than that of CdSe and the optical band edge is expected to be found at a larger wavelength for HgSe NCs than for CdSe NCs at the same particle size. In 2014, ${ }^{34}$ this puzzling observation was clarified by observing the intraband absorption in HgSe NCs. Similar to HgS, ${ }^{33} \mathrm{HgSe} \mathrm{NCs}$ appear to be self doped ${ }^{65}$ (as described in the section dedicated to doping later in this chapter), indicating that doping is realized without the introduction of any impurities while conducting synthesis under the stoichiometric condition (the same molar amounts of $\mathrm{Hg}$ and $\mathrm{Se}$ ). The main evidence for this doping is the presence of a narrow peak in the midinfrared range, as shown in Figure $7 \mathrm{f}$ and $\mathrm{g}$. This peak is the signature of a Fermi level located within the conduction band above the 1Se state, as shown in Figure $12 \mathrm{f}$. Due to this degenerate doping, the interband transition is partly bleached, which considerably smooths the visible and even near-infrared absorption. This lack of clear features in the visible domain and the shifting of the absorption toward the midinfrared range is likely why the intraband absorption was missed in early works on $\mathrm{HgSe}$. Since this observation was made, we observe a complete shift of the motivation for HgSe NC development. The interest in bioapplications appears limited likely due to 
the presence of $\mathrm{Hg}$, with the use of $\mathrm{HgSe}$ particles limited to in-

vitro applications. Nevertheless, many researchers have attempted to integrate HgSe NCs into devices by exploiting their infrared intraband absorption. ${ }^{29,34,39,47,118-120}$

Table 2 summarizes the main synthetic processes used to grow HgSe NCs. The first synthesis of nanosized HgSe involved nearly cluster-sized materials. Similar to the first HgTe synthesis, these syntheses were performed in a polar solvent. Considering the case of HgTe nanocrystals, ${ }^{20}$ Harrison et al. ${ }^{115}$ developed a synthesis technique for HgSe nanocrystals capped with thioglycerol in water. The chalcogenide precursor was obtained from $\mathrm{H}_{2} \mathrm{Se}$ gas, generated ex situ in an adjacent inline flask. Eventually, small HgSe NCs with a PL of approximately $1 \mu \mathrm{m}$ were obtained.

Certain early synthesis approaches for HgSe were partly inspired by bioreactions: HgSe is a common byproduct of the Hg exposure of biological entities through the reaction of Se containing protein with $\mathrm{Hg} .{ }^{54}$ Thus, Kuno et al. ${ }^{116}$ realized the synthesis of a molecular cluster of binary and ternary mercury chalcogenides, including pure HgSe. Clusters were generated using a microemulsion with polyoxyethylene 4-lauryl ether (Brij30) and bis(2-ethylhexyl)sulfosuccinate (AOT) as a surfactant. The clusters were stabilized with a "magic" size and demonstrated optical absorption and photoluminescence at $595 \mathrm{~nm}$ and $628 \mathrm{~nm}$, respectively, as shown in Figure $7 \mathrm{~d}$.

In 2008, the synthesis approach for HgTe proposed by Green et al..$^{93}$ was adapted for HgSe NCs. ${ }^{117}$ TOP:Se was used as a selenium precursor that reacted with mercury acetate in a mixture of TOPO and ethanol. Surprisingly, the 4.9 nm nanocrystals exhibited a band edge at $2.5 \mathrm{eV}$ without any clear exitonic peaks, as shown in Figure $7 \mathrm{a}-\mathrm{c}$, indicating that the IR absorption of this material was likely missed in the early work

The use of a long chain and coordinating amine, such as oleylamine, is critical to synthesize well-defined HgSe nanocrystals. Deng et al. $^{34}$ described the synthesis of HgSe nanocrystals with a low size dispersion through the reaction of mercury chloride with seleneourea in oleylamine. The typical reaction temperature was approximately $100{ }^{\circ} \mathrm{C}$ (between 60 and $150{ }^{\circ} \mathrm{C}$ ). Temperature was the main parameter driving the final particle size. At this stage, the surface chemistry of the particle consisted of oleylammonium chloride, ${ }^{109}$ which could be replaced by thiol either through quenching of the reaction or by a postsynthesis treatment. Moreover, the reaction was generally conducted under stoichiometric conditions or with a slight $\mathrm{Hg}$ excess to favor the doping level and carrier mobility. ${ }^{121}$ The size of the nanocrystals (5.5-6.2 nm) was similar to that of the nanocrystals obtained through TOPO with TOP:Se. ${ }^{117}$ However, Deng et al. demonstrated that an intraband transition occurred in the mid-IR range (4 $\mu \mathrm{m})$. It is likely that the particles obtained using Howes' method (mercury acetate/TOPO/ethanol reacting with TOP:Se) presented intraband absorption and that this aspect was missed by the authors. The process used by Deng et al. ${ }^{34}$ was clearly derived from Keuleyan's procedure for $\mathrm{HgTe} .{ }^{95}$ In the latter procedure, the $\mathrm{HgCl}_{2}$ in oleylamine was used as the reacting medium. Because TOP:Se is less reactive than TOP:Te, the authors replaced the Se precursor with a more reactive species, such as seleneourea. Lhuillier et al. ${ }^{29}$ used the opposite approach; instead of increasing the reactivity of the Se precursor, TOP:Se was used as the precursor, and a more reactive form of mercury, such as mercury oleate, was employed. The latter can be easily prepared by mixing mercury acetate in the presence of oleate. This compound however degrades at high temperatures (more than or equal to $150{ }^{\circ} \mathrm{C}$ ). Under such conditions, in the presence of an amine, $\mathrm{Hg}^{+11}$ is easily reduced to metal $\mathrm{Hg}$. The temperature and duration of the reaction were the two main parameters to tune the particle size. However, degradation of the Hg precursor likely hindered the generation of large NCs. To overcome this difficulty, the authors recommended the use of a phosphine free synthesis, in which the Se precursor was derived from $\mathrm{SeS}_{2}$ diluted in oleylamine. Owing to the lack of phosphine, a larger amount of the nanoparticle surface was available, which facilitated growth, leading to particle sizes of up to $40 \mathrm{~nm}$. This large size tunability allowed the tuning of the energy of the intraband feature from 3 to $25 \mu \mathrm{m}$, as shown in Figure $7 \mathrm{~g} .{ }^{29,122}$

The shape of the HgSe NCs is spherical (Figure 7e), in contrast to that of HgTe, for which most of the synthesis approaches lead to faceted objects. The resulting NCs appear to be relatively monodispersed and easily self organize on TEM grids to form a close packed lattice. Nevertheless, recent transport measurements have indicated that the remaining size distribution limits mobility in HgSe NC arrays, ${ }^{121}$ requiring the development of more effective synthesis techniques. 

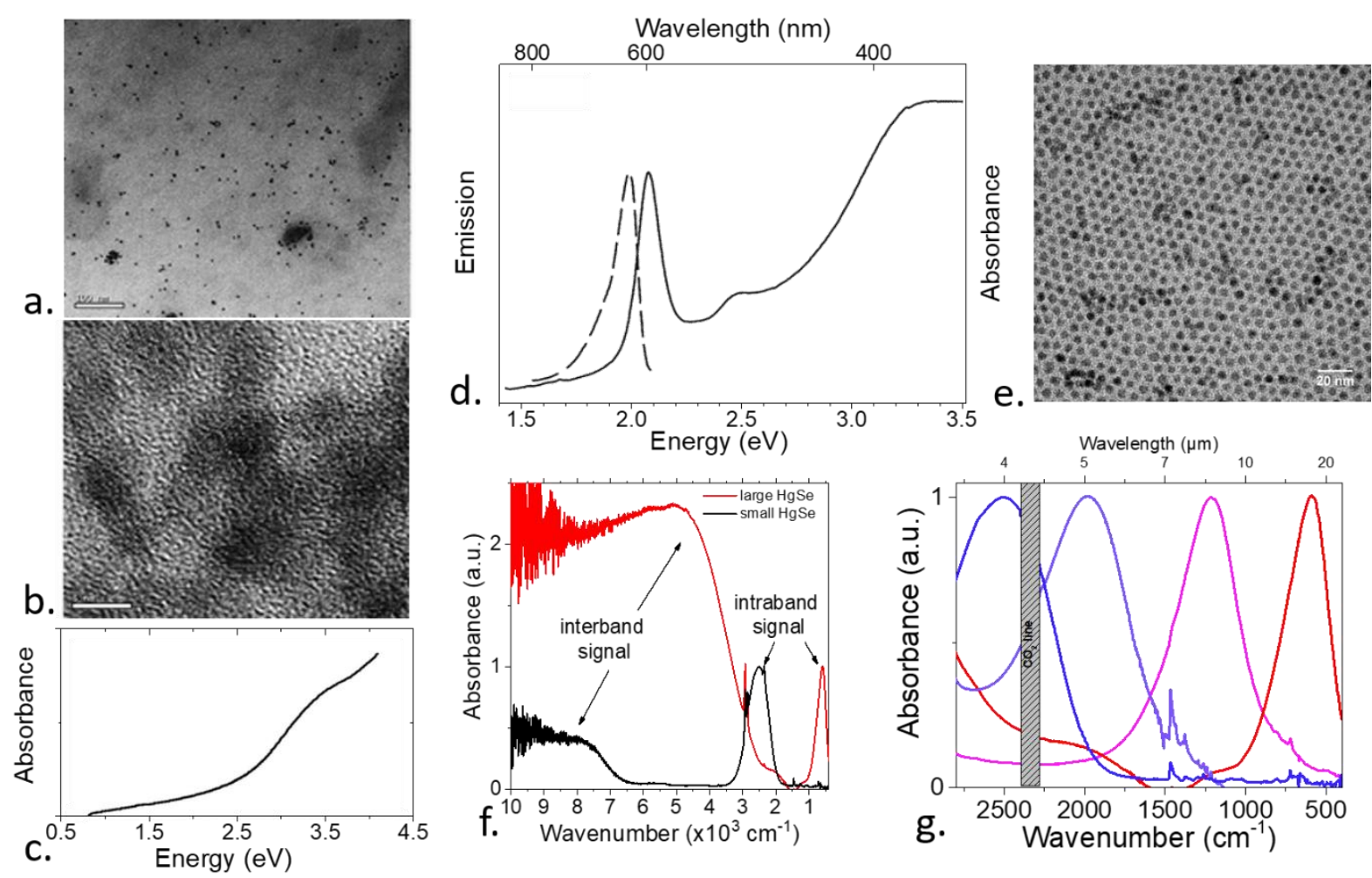

Figure $7 a$. and b. TEM images of HgSe nanocrystals made with mercury acetate and TOP:Se in TOPO. $c$. Corresponding visible to near IR absorption spectrum. Parts a-c are used with permission of the Royal Society of Chemistry, from ref ${ }^{117}$; permission was conveyed through the Copyright Clearance Center, Inc. d. Absorption (full line) and photoluminescence (dashed line) spectra of HgSe clusters prepared in the microemulsion. Part $d$ is adapted with permission from ref ${ }^{116}$ Copyright (2003) American Chemical Society. e. TEM image of typical HgSe nanocrystals synthesized in oleic acid and oleylamine. f. FTIR spectra of small and large HgSe nanocrystals. g. Intraband absorption range of $\mathrm{HgSe}$ nanocrystal obtained from this synthesis. Figures e-g are adapted with permission from ref ${ }^{29}$ Copyright (2016) American Chemical Society.

Table 2 Conditions related to the selection of the relevant synthesis techniques for HgSe NCs.

\begin{tabular}{|c|c|c|c|c|c|c|c|}
\hline $\begin{array}{l}\mathrm{Hg} \\
\text { Precursors }\end{array}$ & $\begin{array}{l}\text { Se } \\
\text { precursor } \\
\text { S }\end{array}$ & Ligands & $\begin{array}{l}\text { Solvents / } \\
\text { Temperature }\end{array}$ & Method & Size $(\mathrm{nm})$ & $\begin{array}{l}\text { Optical } \\
\text { properties }\end{array}$ & $\begin{array}{l}\text { Year/Ref } \\
\text { erence }\end{array}$ \\
\hline $\mathrm{Hg}\left(\mathrm{ClO}_{4}\right)_{2}$ & $\mathrm{H}_{2} \mathrm{Se}$ & thioglycerol & $\begin{array}{l}\mathrm{H}_{2} \mathrm{O}, \\
\text { thioglycerol } \\
\mathrm{T}_{\text {room }}\end{array}$ & & & $\begin{array}{r}1 \mu \mathrm{m} \\
1 \mu \mathrm{m}(\mathrm{PL})\end{array}$ & $2000 / 115$ \\
\hline $\mathrm{Hg}$ (acetate) ${ }_{2}$ & $(\mathrm{TMS})_{2} \mathrm{Se}$ & thioglycerol & $\begin{array}{l}\mathrm{H}_{2} \mathrm{O}, \\
\text { thioglycerol, } \\
\text { hexane, TOP, } \\
\mathrm{C}_{12} \mathrm{E}_{4}, \mathrm{AOT} / \\
\mathrm{T}_{\text {room }}\end{array}$ & Microemulsion & $2-3$ & $\begin{array}{c}595 \mathrm{~nm} \\
620 \mathrm{~nm}(\mathrm{PL})\end{array}$ & $2003 / 116$ \\
\hline $\mathrm{Hg}$ (acetate) ${ }_{2}$ & TOP:Se & & $\begin{array}{l}\text { TOPO, } \\
\text { ethanol / } \\
\text { Troom }\end{array}$ & & $3.9-6$ & $350 \mathrm{~nm}$ & $2008 / 117$ \\
\hline $\mathrm{HgCl}_{2}$ & $\mathrm{Se}=\mathrm{C}\left(\mathrm{NH}_{2}\right)_{2}$ & DDT & OLA $/ 110^{\circ} \mathrm{C}$ & Hot injection & $5.5-6.2$ & $4 \mu \mathrm{m}$ & $2014 / 34$ \\
\hline $\mathrm{Hg}$ (acetate) ${ }_{2}$ & Se-ODE & & $\begin{array}{l}\text { OA, ODE } \quad / \\
100 \\
{ }^{\circ} \mathrm{C} \text {, } \\
\text { Troom and }-78 \\
{ }^{\circ} \mathrm{C}\end{array}$ & & $15-100$ & $\begin{array}{l}800-1000 \mathrm{~nm} \\
1-1.3 \mu \mathrm{m}(\mathrm{PL})\end{array}$ & $2014 / 123$ \\
\hline $\mathrm{Hg}(\text { acetate })_{2}$ & $\begin{array}{l}\text { Cation } \\
\text { exchange } \\
\text { on CdSe } \\
\end{array}$ & $\begin{array}{l}\text { OA, OLA, } \\
\text { DDT }\end{array}$ & $\begin{array}{c}\text { Hexane, } \\
\text { octylamine, }\end{array}$ & Cation exchange & $\begin{array}{c}2-3 \\
\text { atomic } \\
\mathrm{ML}\end{array}$ & $700-800 \mathrm{~nm}$ & $2016 / 31$ \\
\hline
\end{tabular}




\begin{tabular}{|c|c|c|c|c|c|c|c|}
\hline & & & $\begin{array}{l}\text { trioctylamine, } \\
\text { OLA / Troom }\end{array}$ & & $100 \times 200$ & & \\
\hline $\mathrm{Hg}(\text { acetate })_{2}$ & $\begin{array}{l}\text { TOP:Se } \\
\mathrm{SeS}_{2}\end{array}$ & DDT & $\begin{array}{l}\text { OA, OLA / 60- } \\
120^{\circ} \mathrm{C}\end{array}$ & Hot injection & $6-23$ & $4-20 \mu \mathrm{m}$ & $2016 / 29$ \\
\hline $\mathrm{HgCl}_{2}$ & $\begin{array}{l}\text { Cation } \\
\text { exchange } \\
\text { on CdSe }\end{array}$ & TOPO & $\begin{array}{l}\text { ODE, OLA / } \\
150^{\circ} \mathrm{C}\end{array}$ & Cation exchange & 11.3 & $5 \mu \mathrm{m}$ & $2017 / 124$ \\
\hline $\mathrm{HgCl}_{2}, \mathrm{Hgl}_{2}$ & Se & DDT & OLA / $250^{\circ} \mathrm{C}$ & $\begin{array}{l}\text { Injection of both } \\
\text { cation and anion in } \\
\text { hot solvent }\end{array}$ & $20-100$ & 9-18 $\mu \mathrm{m}$ & $2018 / 30$ \\
\hline
\end{tabular}

\subsection{HgS nanocrystal synthesis}

$\mathrm{HgS}$ exhibits two key differences against HgTe and HgSe: (i) the metacinnabar ( $\beta$-HgS) zinc blende structure of mercury sulfide is a narrow bandgap ${ }^{68}(0.65 \mathrm{eV})$ semiconductor, while $\mathrm{HgTe}$ and $\mathrm{HgSe}$ are semimetals. Despite this bandgap, the optical properties of this phase are highly similar to those of $\mathrm{HgSe}$, exhibiting intraband absorption (Figure $8 \mathrm{c}$ ) due to self doping. In addition, (ii) in the bulk and nanoscale, mercury sulfide is stable in two crystal structures. In the second structure, the cinnabar phase ( $\alpha$-HgS) exhibits a bulk bandgap of $2.1 \mathrm{eV}$ and is known as vermilion due to its red color.

\subsection{1. $\beta$-HgS nanocrystal synthesis}

Table 3 summarizes the main synthetic processes used to grow $\beta$-HgS NCs. As in the case of HgSe, HgS nanocrystals were first synthesized for the visible-near-infrared range. The first synthesis ${ }^{125-127}$ of $\mathrm{HgS}$ nanosized materials was in the form of $\mathrm{CdSe} / \mathrm{HgS} / \mathrm{CdS}$ heterostructured quantum dots. The core of CdSe was coated with a few layers of HgS and capped with CdS, thereby achieving a kind of spherical quantum well heterostructure. The CdS quantum dots were formed in the presence of sodiumpolyphosphate by reacting $\mathrm{H}_{2} \mathrm{~S}$ gas with $\mathrm{Cd}\left(\mathrm{ClO}_{4}\right)_{2}$ in water. The $\mathrm{HgS}$ quantum well was grown with $\mathrm{Hg}(\mathrm{ClO})_{2}$ with the same sulfur precursor. Next, the reaction with $\mathrm{Cd}\left(\mathrm{ClO}_{4}\right)_{2}$ was repeated for the last layer. The number of $\mathrm{HgS}$ layers (from 1 to 4 ) sandwiched between CdS could be increased by increasing the deposition cycles. The particle band edge was red shifted from 470 $\mathrm{nm}$ to $800 \mathrm{~nm}$ for larger heterogenous materials. Later, Dorfs et al. ${ }^{128}$ enhanced the complexity of this heterogenous structure by proposing the synthesis of multilayered spherical $\mathrm{CdS} / \mathrm{HgS}$ nanocrystals. The key concept was to increase the thickness of CdS materials before growing the HgS shell to reach partial substitution of the CdS layer by HgS. In 2002, Higginson et al. ${ }^{129}$ developed a synthesis technique for small $\mathrm{HgS}$ nanocrystals. The use of a strongly binding ligand, such as thioglycerol for $\mathrm{Hg}^{2+}, \mathrm{and}$ compartmentalization of the $\mathrm{Hg}$ and $\mathrm{S}$ precursors were crucial to realize controlled growth of $\mathrm{HgS}$ nanocrystals. HgS growth was stopped by adding a cadmium or zinc precursor, which increased the photoluminescence of the materials (QY of 5 to $6 \%$ ) compared to that of the materials passivated using aqueous precursors $(Q Y<1 \%)$. The size range of the resulting materials was from 1 to $5 \mathrm{~nm}$. As shown in Figure 8a, the corresponding absorption spectra exhibited resolved excitonic features, and band edge absorption appeared between 500 and $800 \mathrm{~nm}$. Owing to the $\mathrm{Cd}$ and $\mathrm{Zn}$ passivation, the HgS nanocrystals exhibited clear photoluminescence in the visible range, as shown in Figure 8b. An alternative approach to synthesize aggregated HgS nanocrystals was proposed by Yang et al. ${ }^{130}$, who exploited the fact that ordered two-dimensional arrays of aggregates could be obtained at the interface between water and chloroform. $\mathrm{HgS}$ nanocrystals were formed through the interfacial reaction between $\mathrm{Hg}(\mathrm{acetate})_{2}$ and $\mathrm{H}_{2} \mathrm{~S}$ gas from the upper aqueous phase. The Langmuir monolayer of arachidic acid was used to create aggregates between this surfactant and the HgS nanocrystals. The aggregate size was approximately $260 \mathrm{~nm}$, with the HgS nanocrystals having a size of $8 \mathrm{~nm}$.

The first synthesis using TOP:S as a chalcogenide precursor was proposed by Wichiansee et al. ${ }^{131}$ in 2011 . To overcome the low reactivity of TOP:S compared to TOP:Te and TOP:Se for TOPO-capped HgTe and HgSe synthesis, HgS nanocrystals were synthesized at $120^{\circ} \mathrm{C}$ instead of near room temperature. The resulting nanocrystal solution was polydisperse, with a mean value of $3.9 \mathrm{~nm}$. This polydispersity led to a broad band edge at $910 \mathrm{~nm}$ with a weak and broad photoluminescence from 900 to $1600 \mathrm{~nm}$. Other near-infrared photoluminescence materials were obtained with glutathione-coated HgS nanocrystals. ${ }^{132}$ In this synthesis, the mercury precursor $\left(\mathrm{Hg}\left(\mathrm{ClO}_{4}\right)_{2}\right)$ was dissolved with glutathione in water. $\mathrm{HgS}$ was obtained via the slow injection of $\mathrm{Na} 2 \mathrm{~S}$, and the obtained material exhibited an excitonic shoulder at $781 \mathrm{~nm}$ with photoluminescence at $915 \mathrm{~nm}$. The photoluminescence was maximum at $\mathrm{pH}$ 9.5, which corresponds to the deprotonated form of glutathione; in this state, most of the thiol groups efficiently bind the $\mathrm{Hg}$ on the nanocrystal surface, reducing the impact of the surface traps.

Two synthesis approaches using proteins were proposed for HgS. First, Zhang et al. ${ }^{133}$ used lysozyme as ligands for HgS nanocrystals: $\mathrm{HgCl}_{2}$ dissolved with lysozyme in water reacted with the $\mathrm{S}^{2-}$ provided by thioacetamide at $37^{\circ} \mathrm{C}$. The second approach was proposed by Goswami et al. ${ }^{134}$ In this synthesis, HgS nanocrystals were obtained using the biomineralization process. In the 
first step, $\mathrm{HgO}$ nanocrystals were obtained using mercury nitrate and bovine serum albumin (BSA) in water. $\mathrm{Next}$, $\mathrm{Na}{ }_{2} \mathrm{~S}$ was used for the sulfidation of $\mathrm{HgO}$ to $\mathrm{HgS}$ nanocrystals. The resulting $\mathrm{HgS}$ nanocrystals exhibited a clear excitation peak from 480 to 600 $\mathrm{nm}$, with photoluminescence from 680 to $780 \mathrm{~nm}$. The nanocrystal size was controlled through the $\mathrm{Hg} / \mathrm{S}$ ratio. Later, Han et al. ${ }^{135}$ adapted the phase transfer synthesis from gold to HgS nanocrystals. Mercury was transferred from the aqueous phase to the organic phase by dissolving mercury nitrate and dodecylamine in water/ethanol. The ions were transferred by mixing the aqueous phase with toluene, oleylamine or oleic acid. Sulfidation was achieved by adding elemental sulfur to the organic phase for 3-4 $\mathrm{h}$. The size of the $\mathrm{HgS}$ nanocrystals ranged from $12.5 \mathrm{~nm}$ to $56 \mathrm{~nm}$ depending of the sulfidation temperature and organic phase.

The first study that reported the synthesis of HgS nanocrystals with intraband transition was that of Jeong et al. ${ }^{33}$ This synthesis was adapted from that of $\mathrm{HgTe}$ in oleylamine. However, because TOP:S exhibited an extremely low reactivity with $\mathrm{HgCl}_{2}$, more reactive thioacetamide $\left((\mathrm{TMS})_{2} \mathrm{~S}\right.$, or $\left.\left(\mathrm{NH}_{4}\right)_{2} \mathrm{~S}\right)$ chalcogenide precursors were used. The reaction was quenched using dodecanethiol (DDT). The obtained HgS nanocrystals had a size of $5 \mathrm{~nm}$, and a clear intraband absorption peak was observed in the midinfrared range $(5 \mu \mathrm{m})$. Later, the size ranges of $\mathrm{HgS}$ nanocrystals with intraband transition were expanded in the study of Shen et al. ${ }^{136} \mathrm{The}$ $\mathrm{HgCl}_{2}$ precursor was reacted with $\left(\mathrm{NH}_{4}\right)_{2} \mathrm{~S}$, and the $\mathrm{Hg} / \mathrm{S}$ ratio allowed size control from 2.9 to $14.5 \mathrm{~nm}$, as shown in Figure $8 \mathrm{e}$. As shown in Figure $8 \mathrm{~d}$, the interband transition was slightly modulated by the nanocrystal size compared to the intraband absorption that covered the midinfrared range from 4 to $10 \mu \mathrm{m}$ with corresponding photoluminescence from $3.6 \mu \mathrm{m}$ to $6.7 \mu \mathrm{m}$. Later, Goubet et $a .^{30}$ expanded the size control of several tens of nanometer nanocrystals from 50 to $150 \mathrm{~nm}$ by injecting a mixture of mercury halogenide and elementary sulfur in hot oleylamine. This last synthesis expanded the intraband absorption up to $18 \mu \mathrm{m}$.
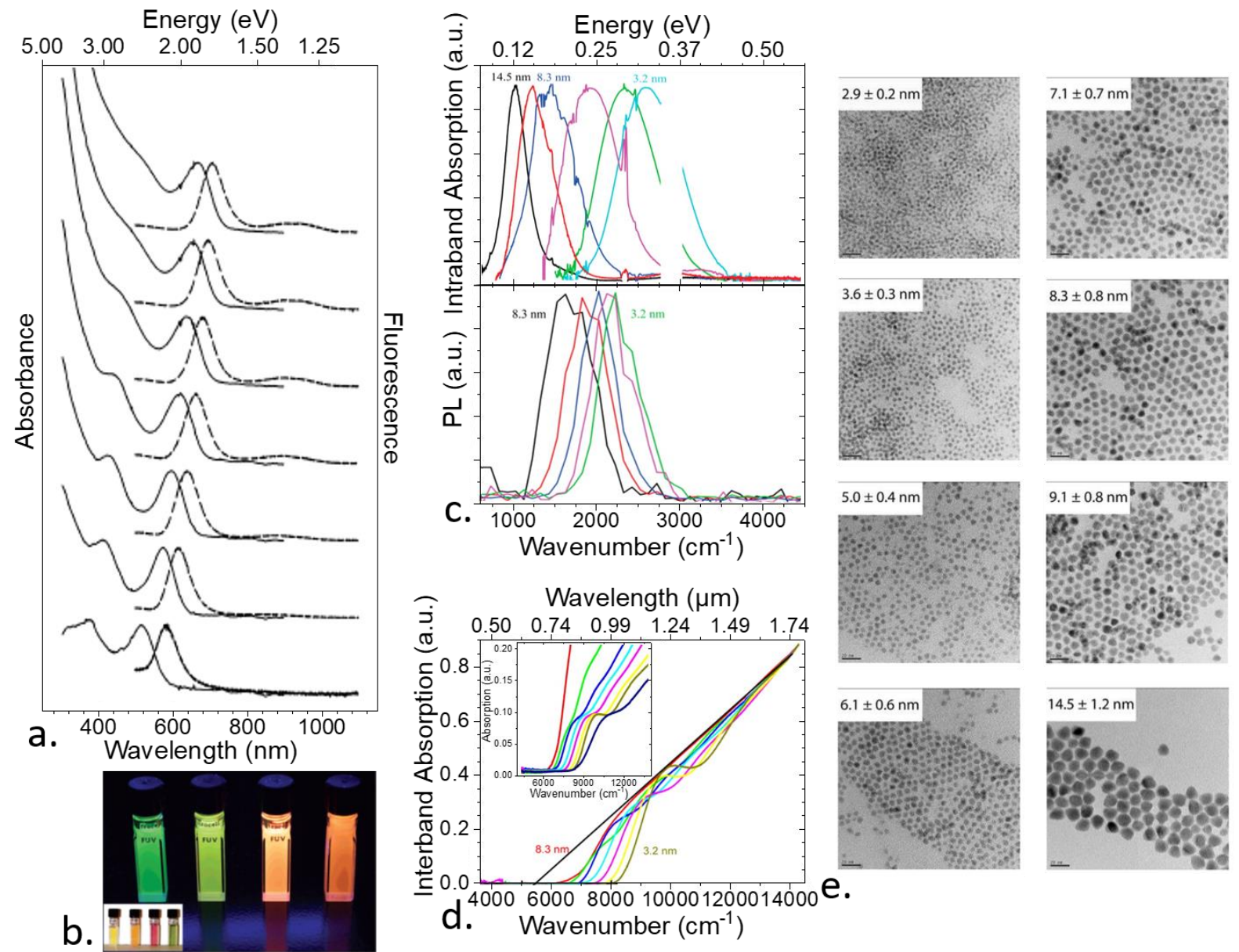

Figure 8 a. Photoluminescence and absorption spectra of $\beta$-HgS nanocrystals during their formation. b. $\beta$-HgS nanocrystals under UV illumination and normal illumination (inset). Parts $a$ and $b$ are adapted with permission from ref ${ }^{129}$ Copyright (2002) American Chemical Society. C. Intraband absorption and photoluminescence of $\beta-\mathrm{HgS}$ nanocrystals with different sizes. $d$. Interband absorption of $\beta$-HgS nanocrystals with various sizes. e.TEM images of $\beta-H g S$ nanocrystals with various sizes. Parts $c-e$ are adapted with permission from ref ${ }^{136}$ Copyright (2016) American Chemical Society.

Table 3 Conditions related to the selection of the relevant synthesis technique for $\beta$-HgS NCs. 


\begin{tabular}{|c|c|c|c|c|c|c|c|}
\hline $\begin{array}{l}\mathrm{Hg} \\
\text { precursor }\end{array}$ & S Precursors & Ligands & $\begin{array}{l}\text { Solvents } \\
\text { temperature }\end{array}$ & Method & $\begin{array}{l}\text { Size } \\
(\mathrm{nm})\end{array}$ & $\begin{array}{l}\text { Optical } \\
\text { properties }\end{array}$ & $\begin{array}{l}\text { Year/ } \\
\text { Ref }\end{array}$ \\
\hline $\mathrm{Hg}\left(\mathrm{ClO}_{4}\right)_{2}$ & $\mathrm{H}_{2} \mathrm{~S}$ & CdS & Water / Troom & Cation exchange & $\begin{array}{l}1-3 \\
\text { mon } \\
\text { olaye } \\
\text { rs }\end{array}$ & $\begin{array}{l}500-800 \mathrm{~nm} \\
713-961 \mathrm{~nm} \\
(\mathrm{PL})\end{array}$ & $\begin{array}{l}1994 / \\
137\end{array}$ \\
\hline $\mathrm{Hg}(\text { acetate })_{2}$ & $(\mathrm{TMS})_{2} \mathrm{~S}$ & $\begin{array}{l}\text { Thioglycerol, } \\
\text { thiophenol, } \\
\text { dodecylamin } \\
\text { e, } \\
\text { hexadecanet } \\
\text { hiol }\end{array}$ & $\begin{array}{l}\text { Cyclohexane, } \\
\text { hexane, } \quad \mathrm{H}_{2} \mathrm{O} \text {, } \\
\text { thioglycerol / Troom }\end{array}$ & & $1-5$ & $\begin{array}{l}500-800 \mathrm{~nm} \\
600-700 \mathrm{~nm} \\
(\mathrm{PL})\end{array}$ & $\begin{array}{l}2002 / \\
129\end{array}$ \\
\hline $\mathrm{Hg}(\text { acetate })_{2}$ & TOP:S & TOP/TOPO & TOPO / $150^{\circ} \mathrm{C}$ & Hot injection & 4 & $\begin{array}{l}\text { Broad } 900 \\
\mathrm{~nm} \\
\text { Broad } 1127 \\
\mathrm{~nm}(\mathrm{PL})\end{array}$ & $\begin{array}{l}2011 / \\
131\end{array}$ \\
\hline $\mathrm{Hg}\left(\mathrm{ClO}_{4}\right)_{2}$ & $\mathrm{Na}_{2} \mathrm{~S}$ & glutathione & $\begin{array}{l}\mathrm{H}_{2} \mathrm{O}, \text { glutathione / } \\
\mathrm{T}_{\text {room }}\end{array}$ & & 2.87 & $\begin{array}{l}700-800 \mathrm{~nm} \\
775-1041 \\
\mathrm{~nm}(\mathrm{PL}) \\
\end{array}$ & $\begin{array}{l}2012 / \\
132\end{array}$ \\
\hline $\mathrm{HgCl}_{2}$ & $\begin{array}{l}\text { thioacetamid } \\
\mathrm{e}\end{array}$ & Lysozyme & $\begin{array}{l}\mathrm{H}_{2} \mathrm{O} \text {, lysozyme / } 37 \\
{ }^{\circ} \mathrm{C}\end{array}$ & $\begin{array}{l}\text { Lypozyme } \\
\text { template }\end{array}$ & $\begin{array}{l}14- \\
27\end{array}$ & $\begin{array}{l}400 \quad \mathrm{~nm} \\
470 \mathrm{~nm}(\mathrm{PL})\end{array}$ & $\begin{array}{l}2012 / \\
133\end{array}$ \\
\hline $\mathrm{Hg}\left(\mathrm{NO}_{3}\right)_{2}$ & $\mathrm{Na}_{2} \mathrm{~S}$ & BSA & $\begin{array}{l}\mathrm{H}_{2} \mathrm{O}, \mathrm{NaOH}, \mathrm{BSA} / \\
\mathrm{T}_{\text {room }}\end{array}$ & $\begin{array}{l}\text { Biomineralizatio } \\
\mathrm{n}\end{array}$ & 3.8 & $\begin{array}{l}480-600 \mathrm{~nm} \\
680-800 \mathrm{~nm} \\
(P L)\end{array}$ & $\begin{array}{l}2012 / \\
134\end{array}$ \\
\hline $\mathrm{HgCl}_{2}$ & $\begin{array}{l}\text { thioacetamid } \\
\text { e, } \quad(\mathrm{TMS})_{2} \mathrm{~S} \text {, } \\
\left(\mathrm{NH}_{4}\right)_{2} \mathrm{~S}\end{array}$ & DDT & OLA $/ 30^{\circ} \mathrm{C}$ & Injection & $\begin{array}{l}3.5- \\
14\end{array}$ & $\begin{array}{l}5 \mu \mathrm{m} \\
5 \mu \mathrm{m}\end{array}$ & $\begin{array}{l}2014 / \\
33\end{array}$ \\
\hline $\mathrm{Hg}\left(\mathrm{NO}_{3}\right)_{2}$ & $\mathrm{~s}$ & $\begin{array}{l}\text { DDA, OLA or } \\
\text { OA }\end{array}$ & $\begin{array}{l}\text { Toluene, OLA, OA, } \\
\text { DDA / Troom or } 80^{\circ} \mathrm{C}\end{array}$ & Phase transfer & $\begin{array}{l}12- \\
100\end{array}$ & & $\begin{array}{l}2014 / \\
135\end{array}$ \\
\hline $\mathrm{HgCl}_{2}$ & $\left(\mathrm{NH}_{4}\right)_{2} \mathrm{~S}$ & $\begin{array}{l}\text { OLA, TOP or } \\
\text { DDT }\end{array}$ & $\begin{array}{l}\text { OLA, TCE, TOP / } \\
\text { Troom }\end{array}$ & & $3-15$ & $\begin{array}{l}4-10 \mu \mathrm{m} \\
3.6-6.7 \quad \mu \mathrm{m} \\
(\mathrm{PL})\end{array}$ & $\begin{array}{l}2016 / \\
136\end{array}$ \\
\hline $\mathrm{HgCl}_{2}, \mathrm{Hgl}_{2}$ & $S$ & DDT & OLA / $250^{\circ} \mathrm{C}$ & $\begin{array}{l}\text { Injection of both } \\
\text { cation and anion } \\
\text { in hot solvent }\end{array}$ & $\begin{array}{l}50- \\
150\end{array}$ & 9 and $18 \mu \mathrm{m}$ & $\begin{array}{l}2018 / \\
30\end{array}$ \\
\hline
\end{tabular}

\subsection{2. $\alpha-H g S$ nanocrystal synthesis}

Cinnabar crystallizes in the trigonal system with a noncentrosymmetric structure. Specifically, there are two enantiomorphic space groups $\left(P 3_{1} 21\right.$ and $\left.P 3_{2} 21\right)$ in this material (see Figure $\left.9 \mathrm{~b}\right)$. Ben-Moshe et al. ${ }^{138}$ were pioneers of the use of this structure and proposed the synthesis of intrinsically chiral HgS nanocrystals. In the first step, $\beta$-HgS nanocrystals were obtained by reacting mercury nitrate with thioacetamide in the presence of penicillamine in water. By using enantiomeric penicillamine, $\mathrm{HgS}$ growth could be directed to one space group of $\alpha-\mathrm{HgS}$ after a few hours of reaction. The structural transition from $\beta$ to $\alpha$ could be observed as the color changed from black to red, the color of the vermilion. As shown in Figure 9a, the long and short axes of the elongated nanocrystals were sized 15 and $10 \mathrm{~nm}$, respectively. The optical activity of $\alpha-\mathrm{HgS}$ was characterized by circular dichroism (see Figure 9c). At $550 \mathrm{~nm}$, the band edge exhibited clear optical activity depending on the enantiomeric mixture of the capping penicillamine. The resulting dissymmetry factor $\left(10^{-2}\right)$ was one order of magnitude larger than that of the achiral nanocrystals coated with chiral ligands. Later, Wang et al. ${ }^{139}$ proposed a synthesis of pure $\mathrm{HgS}$ enantiomorphs. A pronounced helicoidal chiral shape was obtained after successive growth of $\alpha-\mathrm{HgS}$ (see Figure $9 \mathrm{~d}-\mathrm{f}$ ). Seeds are grown by reacting mercury nitrate and thioacetamide in the presence of penicillamine (either D or L enantiomers). A second epitaxial growth was realized with the injection of the same precursors through a syringe pump.

Figure $9 \mathrm{~g}$ and $\mathrm{h}$ show the oriented attachment of the left- and right-handed helicoidal $\alpha$-HgS nanocrystals, respectively. Wang et al. ${ }^{139}$ demonstrated that the alignment of the chiral nanocrystals enhanced the optical activity of the material compared to that of the individual nanocrystals. Several studies ${ }^{140,141}$ demonstrated that a slight modification of the chemical structure but not the stereochemistry induced reversed optical activity of the $\alpha$-HgS nanocrystals. 

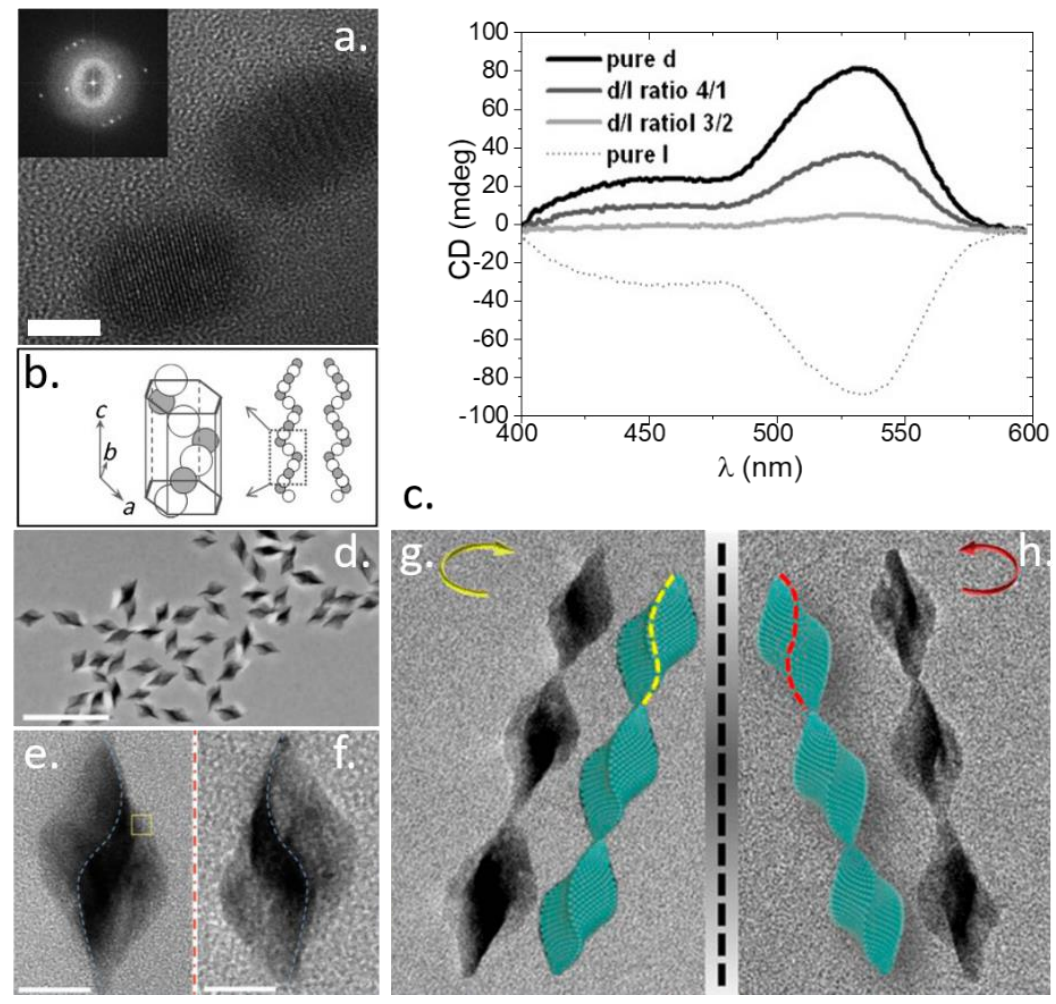

Figure 9 a. High resolution TEM image of $\alpha$-HgS nanocrystals. b. Atomic model of the cinnabar $\mathrm{HgS}$ lattice. c. Circular dichroism (CD) spectra of $\alpha$-HgS nanocrystals with different $D / L$ ratios of penicillamine. Parts a-c are adapted with permission from ref ${ }^{138}$ Copyright (C) 2013 WILEY-VCH Verlag $\mathrm{GmbH} \&$ Co. KGaA, Weinheim. d. TEM image of $\alpha$-HgS nanocrystals synthesized with successful growth. e. and f. TEM image of two hellicoidal enantiomorphs of $\alpha$-HgS. Parts $d-f$ are adapted with permission from ref ${ }^{139} . \mathrm{g}$. and $\mathrm{h}$. TEM images of left- and right-handed oriented attachment of $\alpha-\mathrm{HgS}$ nanocrystals. Parts $g-h$ are adapted with permission from ref ${ }^{142}$ Copyright (2017) American Chemical Society.

\subsection{Shape control and 2D materials}

For cadmium ${ }^{7,143,144}$ and lead chalcogenides ${ }^{145}$, a broad variety of NC shapes can be synthesized; for mercury chalcogenides, more effort has been focused on size control to extend the absorption toward larger wavelengths $s^{3,27-30}$ than on realizing shape control. The shape of the particles is thus dictated by the targeted bandgap. For example, by using the process recommended by Keuleyan et al. ${ }^{95}$ the shape can be changed from small aspect ratio rods to branched particles to tetrahedrons, with bandgaps of $6000 \mathrm{~cm}$ ${ }^{1}, 4000 \mathrm{~cm}^{-1}$, and $2000 \mathrm{~cm}^{-1}$, respectively. Among various efforts, Shen et al. attempted to control the particle shape of the $\mathrm{HgX}$ core nanocrystals. ${ }^{63}$ The authors used $\mathrm{TMS}_{2} \mathrm{Te}$ as a telluride precursor and obtained more spherical particles. Although the initial objective was to reduce material aggregation, ${ }^{63}$ the shape of the NCs likely considerably enhanced the effective carrier mobility, especially for films of spherical NCs compared to branched nanoparticles with similar surface chemistries. ${ }^{36,146}$

The most notable efforts to control the shape of $\mathrm{HgX} \mathrm{NCs}$ were driven by Ithurria and coworkers. This team pioneered the field of $2 \mathrm{D}$ cadmium chalcogenide nanoplatelets (NPLs). ${ }^{8,144}$ Compared to spherical nanocrystals, the specific growth mechanism of NPLs ${ }^{147,148}$ prevented roughness from occurring along the only confined direction. Consequently, no inhomogeneous shape occurred, leading to spectral broadening, and the single particle spectrum overlapped with the ensemble spectrum. ${ }^{149}$ This phenomenon led to a narrower PL signal than that for spherical quantum dots, for which the linewidth was limited by the particle size distribution. The 2D shape also led to a higher exciton binding energy, which increased the radiative rate. The NPLs were thus successfully integrated as active materials to realize stimulated emission, ${ }^{150-152}$ LEDs $^{153-156}$ and other optoelectronic devices, ${ }^{157}$ such as field effect transistors and phototransistors. ${ }^{41,158,159}$ The 2D-shaped particles based on lead chalcogenides were reported with optical absorption in the NIR and SWIR. ${ }^{160}$ However, the corresponding growth mechanism was different (by clustering of the preformed nanocrystals) from that of the CdX NPLs, and narrow optical features were not observed in these PbX NPLs. Consequently, researchers attempted to develop $\mathrm{HgX}$ materials with a clearly defined structure (clearly defined facet) and optimized optical properties (narrower optical feature, faster $\mathrm{PL}$ ).

The growth method of CdX NPLs relies on the formation of NC seeds from cadmium oleate and selenium in a noncoordinating solvent (octadecene). When the seeds reach a critical size, a short ligand is introduced to break the symmetry of the seeds and 
induce 2D growth. ${ }^{147}$ Applying this method while replacing Cd with $\mathrm{Hg}$ has been unsuccessful to date, and no direct synthesis of $\mathrm{HgX}$ NPLs has been reported. Izquierdo et al. ${ }^{161}$ proposed an indirect method to obtain HgX NPLs through cation exchange, as shown in Figure 10a. The authors first synthesized CdSe and CdTe NPLs ${ }^{161}$ and later exposed these NPLs to a Hg precursor. The critical condition to preserve the 2D shape of the NPLs without introducing surface roughness is to ensure slow growth to avoid destabilizing the chalcogenide lattice. ${ }^{161,162}$ If the cation exchange is incomplete, then puddles of $\mathrm{Hg}$ form and dual color emission may occur: the first PL signal comes from CdSe, while the other signal involves $\mathrm{Hg}$ trap states. ${ }^{163}$ In contrast, when sufficient $\mathrm{Hg}$ is introduced in the $\mathrm{Hg}$ oleate form, complete (confirmed through EDX analysis) cation exchange can be realized while preserving the $2 \mathrm{D}$ shape of the particles (see Figure $10 \mathrm{~b}-\mathrm{c}$ ). During the process, absorption occurs owing to the CdX NPLs first disappearing and later reappearing in a strongly redshifted manner (see Figure 10e). Since bulk $\mathrm{HgTe}$ is a gapless semiconductor, all the band edge energy of the NPL $(\approx 1.5 \mathrm{eV})$ can be attributed to the quantum confinement. Consequently, HgTe NPLs are one of the most confined semiconductor NCs. This aspect is of particular interest to explore the dispersion relation far from the Brillouin zone center. The specific signature of this strong confinement in the temperature dependence of the NPL bandgap is discussed later in the section dedicated to the electronic structure. Due to this strong confinement, the exact band edge energy is strongly dependent on the surface chemistry, which reflects the leakage of the wave function outside of the $\mathrm{HgX}$ core. A clear signature of the well-preserved roughness-free NPL shape is observed from the PL linewidth. The PL linewidth (full width at half maximum) is below $60 \mathrm{meV}$ for an emission at $880 \mathrm{~nm}$, narrower than that of a single PbS NC (typically $100 \mathrm{meV}$ ). ${ }^{164}$ In addition, the emission is considerably faster than that reported for other NCs emitting in the near IR, with PL decay times of approximately 50 ns, in contrast to the corresponding value of several microseconds for PbS NCs.
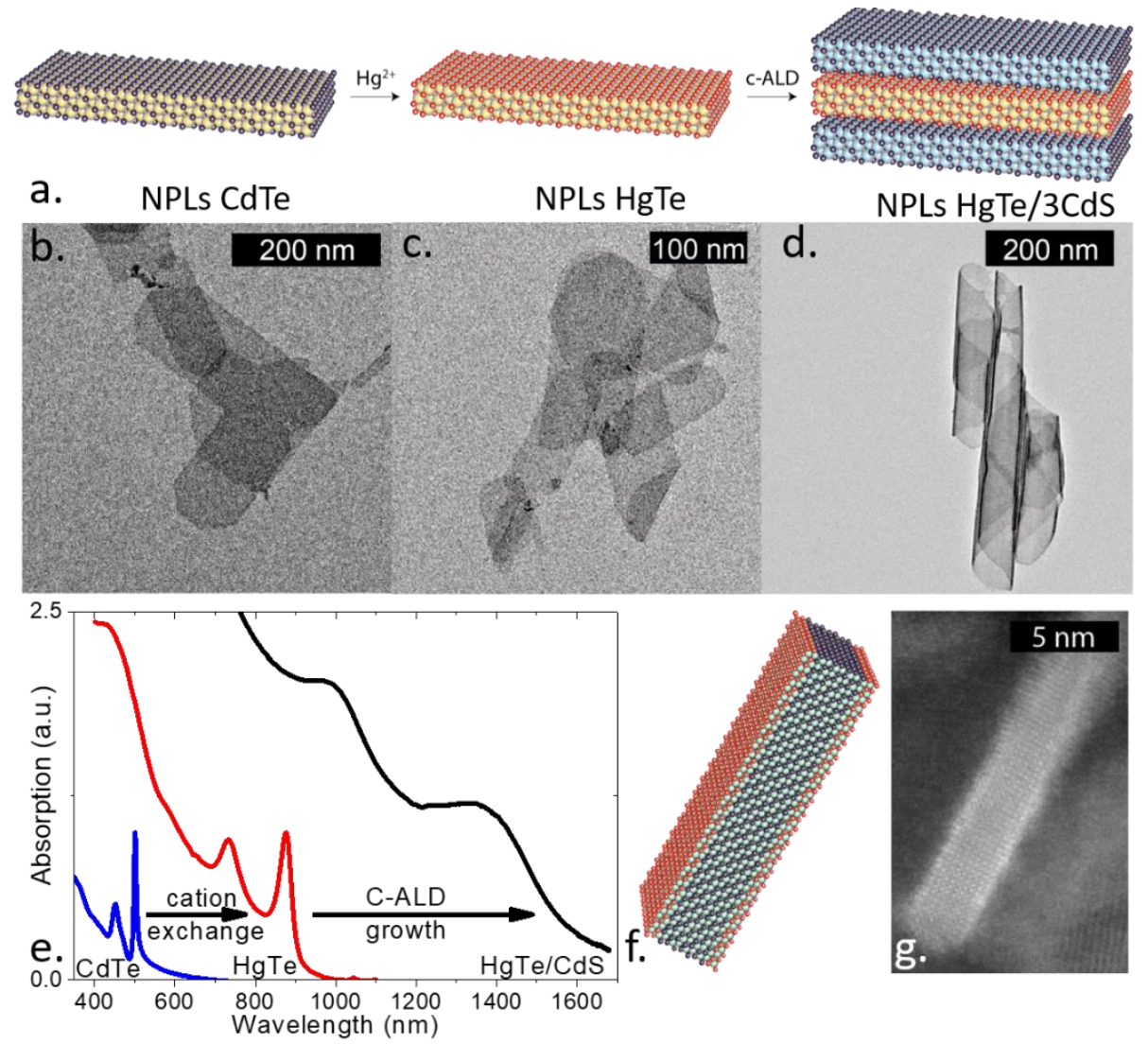

Figure 10 a. Scheme of the growth mechanism used to achieve HgTe/CdS core-shell NPLs. b. TEM image of CdTe NPLs. c. TEM image of HgTe NPLs. d. TEM image of HgTe/3CdS NPLs. e. Absorption spectra of CdTe, HgTe and HgTe/CdS (1 layer of CdS) NPLs. Parts a-e are reproduced from ref ${ }^{165}$ with permission from The Royal Society of Chemistry. f. Scheme of a $10 \mathrm{ML} \mathrm{HgSe/CdSe/HgSe}$ heterostructure. (g) High resolution TEM in the dark field mode of a $10 \mathrm{ML} \mathrm{HgSe} / \mathrm{CdSe} / \mathrm{HgSe}$ heterostructure. Parts $f$ and $g$ are adapted with permission from ref ${ }^{166}$ Copyright (2018) American Chemical Society.

While complete cation exchange occurs for thin NPLs (3 monolayers), the exchange is not complete for thicker NPLs as only the two surface planes of $\mathrm{Cd}$ are exchanged (see Figure $10 \mathrm{f}$ and $\mathrm{g}$ ). This observation fully agrees with previous attempts to conduct cation exchange with mercury on nanocrystals, which exhibited a clear self-limiting process. ${ }^{15,124,167}$ This aspect prevents NPL absorption in the midinfrared region. Nevertheless, an alternative strategy to push the absorption of the NPLs above the telecom 
range $(>1.55 \mu \mathrm{m})$ is to realize additional post-synthesis growth of CdS with c-ALD, as reported by Gréboval et al. ${ }^{165}$ This procedure strongly redshifts the absorption feature of the HgTe NPLs, as shown in Figure 10a, $d$ and e. The method is described in more detail in the next section dedicated to heterostructures. Note that because of the lattice mismatch between the two materials (> $10 \%)$, the NPLs twist (see Figure 10d). Regrettably, the introduction of a shell with a type I band alignment does not improve the $\mathrm{PL}$, but the presence of the shell can extend the wave function delocalization and lead to photoconduction in the shortwave infrared (SWIR) region.

\subsection{Heterostructures}

Even though the efforts to grow mercury chalcogenide NCs are not as notable as those dedicated to Cd-based materials, significant progress has been made. For CdSe, access to the display mass market has been enabled through the growth of heterostructures with enhanced photoluminescence. Similarly, $\mathrm{HgX}$-based heterostructures appear to be promising candidates to realize high IR luminescence and more stable IR absorbers.

The investigation of colloidal mercury chalcogenides started from heterostructures when Eychmüller et al. ${ }^{15}$ proposed growing a HgS quantum well on top of CdS NCs, as discussed later in this section. There are multiple motivations for HgX-based heterostructures. First, owing to the low interband ${ }^{95,168}$ and intraband ${ }^{33,169}$ PL efficiency of IR NCs, ${ }^{170}$ it is desirable to grow type I heterostructures to boost the luminescence yield. The narrow bandgap of $\mathrm{HgX}$ leads to a type I band alignment with most semiconductors. Moreover, the number of nonradiative decay paths, which impede detection owing to the introduction of additional noise, ${ }^{125}$ can be reduced by increasing the quantum yield. In addition, the design of type II band alignment heterostructures is desirable to enhance the exciton dissociation at the nanoparticle level. Another key motivation for HgX-based heterostructures arises from their tendency to sinter even under mild annealing conditions. HgX NCs are mostly grown at low temperatures (60-120 ${ }^{\circ} \mathrm{C}$ ); consequently, when such structures are exposed to similar temperatures, $\mathrm{HgX}$ nanocrystals aggregate. HgTe appear as strongly confined (i.e., exhibit a large Bohr radius of $40 \mathrm{~nm}$ compared to that of $7 \mathrm{~nm}$ for CdSe) gapless material. In the case of a stronger confinement, the electronic structure is more sensitive to size changes. Moreover, in contrast to other semiconductors, the gapless nature of this compound enables the reduction of the NC bandgap to zero. In other words, annealing changes the optical bandgap, which redshifts the cutoff wavelength; moreover, annealing increases the number of thermally activated carriers and thereby the dark current of the device. Consequently, $60^{\circ} \mathrm{C}$ corresponds to the upper temperature limit for the operation of $\mathrm{HgX}$-based optoelectronic devices. Moreover, owing to the low temperature sintering, the diverse fabrication steps typically employed for the encapsulation and protection of nanomaterials or the processing of the resist (i.e., baking step) during the microfabrication procedure cannot be applied in this case. For example, atomic layer deposition, a commonly used protection technique, is generally conducted at $150{ }^{\circ} \mathrm{C}$ for several hours. Overall, the growth of $\mathrm{HgX}$ heterostructures while ensuring both tunable IR optical features and temperature stability appears to be a bottleneck to integrate these IR materials for mass market devices, ${ }^{169}$ beyond the presence of heavy metals.

From a practical perspective, the growth of heterostructures should have benefited from methods developed for other II-VI compounds. However, the growth of $\mathrm{HgX}$-based heterostructures appears to be highly challenging. This difficulty is related to the low temperature growth of $\mathrm{HgX}$ compounds, owing to which the core of the $\mathrm{HgX} \mathrm{NCs} \mathrm{is} \mathrm{incompatible} \mathrm{with} \mathrm{the} \mathrm{high} \mathrm{temperature}$ growth of the shells, which occurs at approximately $250{ }^{\circ} \mathrm{C}$ and above $300{ }^{\circ} \mathrm{C}$ for $\mathrm{CdX}$ materials and zinc-based semiconductors, respectively. Several precursors used for $\mathrm{HgX}$ growth, such as $\mathrm{Hg}$ oleate, also decompose at temperatures above $150{ }^{\circ} \mathrm{C}$. Nevertheless, only a few studies have examined the growth of colloidal HgX heterostructures.

As mentioned previously, the development of HgX nanocrystals was initiated by Weller and coworkers. ${ }^{15,125,171-173}$ The authors performed cation exchange on $\mathrm{CdS}$ nanocrystals with $\mathrm{Hg}^{2+}$ ions, ${ }^{17-19}$ thereby effectively replacing some of the surface $\mathrm{Cd}^{2+}$ with $\mathrm{Hg}$ cations. In this manner, a HgS shell was generated, which behaved as a HgS spherical quantum well due to the type I band alignment with $\mathrm{CdS}^{127}$ as shown in Figure $11 \mathrm{~b}$. The presence of this narrow bandgap material led to a redshift of the optical features, and the absorption reached the red and near IR, ${ }^{127,174}$ considerably below the bulk bandgap of CdS (2.4 eV); see Figure 11a. Later, this process was extended to other chalcogenides to obtain $\mathrm{CdTe} / \mathrm{HgTe}^{32,167,175,176}$ and CdSe/HgSe $\mathrm{H}^{166}$ heterostructures. Despite this early success of cation exchange to grow $\mathrm{HgX}$ heterostructures, the reverse strategy in which the $\mathrm{HgX}$ core is exposed to $\mathrm{Cd}$ atoms has not been extensively investigated. In contrast, in the case of lead chalcogenides, the heating of PbS(e) in the presence of cadmium oleate is the most common strategy to obtain core shell structures ${ }^{177-180}$ likely because of the relatively large size of $\mathrm{Hg}$ atoms, owing to which the introduction or removal of $\mathrm{Hg}$ atoms is more difficult than the removal of smaller atoms.

At present, colloidal atomic layer deposition (c-ALD), ${ }^{181,182}$ as a shell growth method, is the most effective approach to realize CdX shell growth on $\mathrm{HgX}$ cores. The method was initially developed by Talapin and coworkers to grow shells on 2D nanoplatelets ${ }^{67,157,183}$ (NPLs) of cadmium chalcogenides. At that time, attempts to grow shells using the high temperature method developed for spherical quantum dots on CdSe NPLs were inconclusive. The NPL shape was considerably affected by the process, 
owing to which the surface roughness increased and the narrow optical features of the NPLs were lost. c-ALD is a room temperature process and leads to gentler particle surface reorganization. In the case of successive ion layer adsorption and reaction (SILAR), ${ }^{184}$ precursors of cations and anions are introduced successively; however, unlike SILAR, the process is self-limited, similar to conventional ALD. Because the surface of II-VI NCs is generally cation rich, the method starts with the exposure of the nanoparticles to anions. This process is similar to the phase transfer applied for ligand exchange to prepare conductive inks ${ }^{24}$ and is generally conducted in polar solvents even though shell growth can be realized in the nonpolar phase. ${ }^{181}$ Next, the cation precursor is introduced, generally from cadmium halide, for the growth of the $\mathrm{CdX}$ shell. These two steps are repeated as many times as necessary to obtain the targeted shell thickness; see Figure 11c-e. In practice, saturation of the shell growth occurs after 4/5 layers. To grow thicker shells, additional washing steps to remove excess precursors are required.

Because of the broader range of the available sulfide precursors and the possibility of conducting the reaction in air, the method was first applied to the growth of CdS shells on $\mathrm{HgTe}^{33} \mathrm{HgS}^{136}$ and $\mathrm{HgSe}^{185,186}$ cores. In general, the sulfide precursor was $\mathrm{Na}_{2} \mathrm{~S}$ or $\left(\mathrm{NH}_{4}\right)_{2} \mathrm{~S}$. The approach was later extended to chalcogenides, CdSe ${ }^{185}$ and CdTe shells. ${ }^{187}$ For Se and especially for Te, air-free conditions are required. In the case of selenide (or telluride), the precursor is generally obtained from elemental Se (or Te) reduced by $\mathrm{NaBH}_{4},{ }^{188,189}$ although the use of $\mathrm{Na}_{2} \mathrm{Se}$ and $\mathrm{Na}_{2} \mathrm{Te}$ has also been reported. ${ }^{187}$

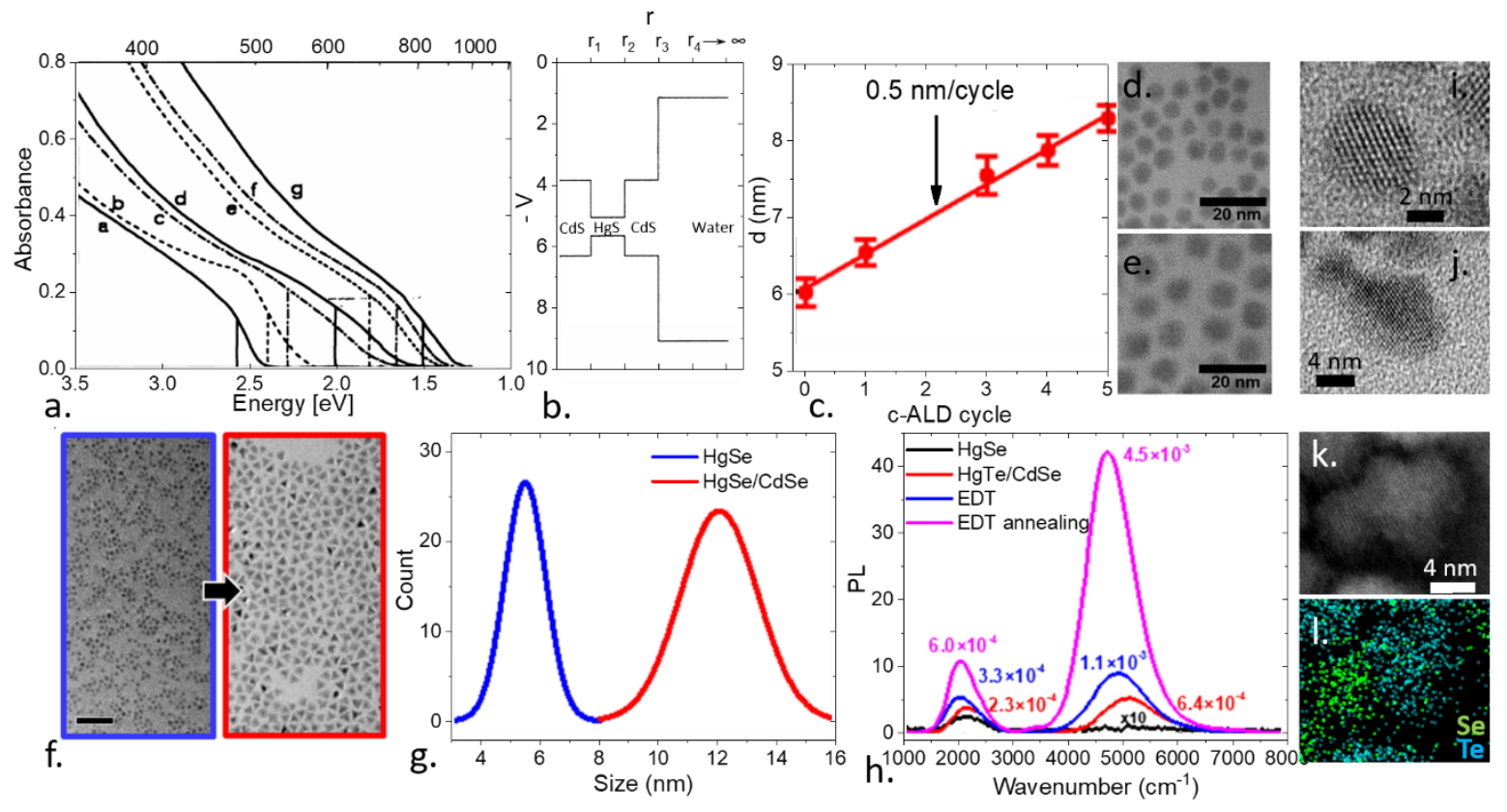

Figure 11 a. Comparison of the absorption spectra of CdS/HgS/CdS heterostructures with various compositions with the theoretical results. b. Potential diagram of the CdS/HgS/CdS heterostructure in water. Parts $a$ and $b$ are reprinted with permission from ref ${ }^{125}$ Copyright (1994) by the American Physical Society. c. Average nanocrystal diameter as a function of the colloidal ALD cycle. The vertical error bars represent the standard deviation of the QD distribution. Bright field TEM image of $6.0 \mathrm{~nm} \mathrm{HgSe} \mathrm{QDs}$ (d.) and HgSe/CdSe QDs (e.) obtained after three C-ALD cycles. Parts c-e are adapted with permission from ref ${ }^{185}$ Copyright (2017) American Chemical Society. $f$. TEM of the (blue) HgSe core and (red) thick $\mathrm{HgSe} / \mathrm{CdSe}$ core/shell (scale bar: $50 \mathrm{~nm}$ ). g. Normal size distributions of the (blue) HgSe core and (red) thick HgSe/CdSe core/shell. $h$. PL spectra of $\mathrm{HgSe} / \mathrm{CdSe}$ films with different treatments measured in air. Parts $f-h$ are adapted with permission from ref ${ }^{187}$ Copyright (2019) American Chemical Society. High resolution TEM images of (i) the HgSe core and (j) $\mathrm{HgSe} / \mathrm{HgTe}$ heterostructure nanocrystals $\mathrm{k}$. HAADF STEM image of a $\mathrm{HgSe} / \mathrm{HgTe}$ heterostructure and (I) localization of the Se and Te, as obtained through EDX mapping. Parts i-I are adapted with permission from ref ${ }^{120}$ Copyright (2018) American Chemical Society.

Methods other than c-ALD have been developed to grow $\mathrm{CdX}$ shells on $\mathrm{HgX}$ cores to overcome the limitations of c-ALD. In the case of CdSe NPLs, it has been observed that materials with c-ALD-grown shells present lower PL efficiencies than those obtained from high temperature shell growth. ${ }^{153,155,156,190}$ This loss of performance can be attributed to the presence of defects in material grown by c-ALD. ${ }^{188,191}$ To date, alternative shell growth has only been reported using HgSe cores. ${ }^{120,169,187}$ To avoid the exposure of HgX nanoparticles to high temperatures, Deng et al. employed mild conditions to grow $\mathrm{CdS} .{ }^{169}$ Shell growth was conducted at $100{ }^{\circ} \mathrm{C}$, and a reactive form of sulfide $\left(\mathrm{H}_{2} \mathrm{~S}\right)$ was used to balance the small growth kinetics induced by the low temperature. Precursors of 
Cd (cadmium oleate) and sulfide were introduced successively for SILAR growth. The growth of a CdSe shell using a high temperature method was reported by Shen et al. ${ }^{187}$ Specifically, shell growth was conducted at $200{ }^{\circ} \mathrm{C}$, while a mixture of cadmium oleate and TOP:Se was slowly injected (see Figure $11 \mathrm{f}-\mathrm{g}$ ). The overall introduction of CdX shells on $\mathrm{HgX}$ core NCs led to no or marginal improvements in the PL signal, ${ }^{33,136,187}$ as shown in Figure $11 \mathrm{~h}$. The reason for this lack of gain in PL efficiency remains unclear.

To date, most efforts have been focused on the growth of a $\mathrm{CdX}$ shell to reduce the number of nonradiative recombination pathways. Goubet et al. explored the growth of a narrow bandgap layer of HgTe on HgSe cores; ${ }^{120}$ see Figure $11 \mathrm{i}-\mathrm{I}$. In this case, shell growth was achieved from the slow injection of mercury iodide and TOP:Te into HgSe cores at low temperatures (60-100 $\left.{ }^{\circ} \mathrm{C}\right)$. The objective was to uncouple the optical absorption and transport properties. The authors designed a heterostructure in which intraband absorption was enabled by the HgSe cores and the HgTe shell facilitated transport. As discussed in the section dedicated to intraband detection, the obtained heterostructure spectrum combined intraband absorption in the HgSe and a type II interband transition with the hole located in HgTe (see Figure 40b). This heterostructure led to an enhanced detection performance of MWIR devices (reduced dark current, increased thermal activation energy and smaller response time) compared with that achieved using the HgSe core only material (see Figure $40 \mathrm{c}$ and d).

\subsection{Doping}

The electronic structure of mercury chalcogenides is often defined in terms of the inverted band ordering and gapless nature of the bulk material. The influence of the narrow bandgap of mercury chalcogenides on the carrier density and, consequently, on the optical and transport properties of such materials has not been extensively discussed. The two latter aspects are discussed later in this review. However, it is critical to understand the origin of the large carrier density in HgX nanocrystals and the mechanism to realize tuning of the doping magnitude.

Specifically, the high carrier density in HgX NCs cannot be attributed to the introduction of extrinsic impurities. The high carrier density results from two processes. The first process is self-doping (i.e., doping that occurs without the introduction of extrinsic impurities), which can lead to degenerate doping. The Fermi level lies in the conduction band, resulting in one or more free carriers per NC. This doping mechanism is specific for narrow bandgap materials, as discussed further in the next section. The second process is observed in small (diameter less than $12 \mathrm{~nm}$ ) HgTe NCs and corresponds to thermal activation. For example, assuming a degeneracy of two for the conduction band $1 \mathrm{~S}$ state and assuming a spherical particle with random close packing (film compactness of 0.64$)$, the population of the $1 S$ state is given by $n_{1 S}=\frac{2 \times 0.64}{4 / 3 \pi R^{3}} \exp \left(-\frac{E_{G}}{2 k_{B} T}\right)$, where $k_{B} T$ is the thermal energy (25 $\mathrm{meV}$ at room temperature). This expression assumes no doping, with the Fermi level positioned in the middle of the bandgap. At $\mathrm{R}=6 \mathrm{~nm}, 10^{16}$ carriers.cm ${ }^{-3}$ (or 1 carrier per $100 \mathrm{NCs}$ ) are obtained for HgTe with a $250 \mathrm{meV}\left(5 \mu \mathrm{m}\right.$ or $2000 \mathrm{~cm}^{-1}$ ) bandgap, while for $\mathrm{CdSe}$, under the same particle size and same degeneracy, 6 carriers. $\mathrm{cm}^{-3}$ (or $9 \times 10^{-18}$ per NC) are obtained. This aspect is a major difference in the two materials: even if a perfectly intrinsic material is assumed, the carrier density of HgTe is $10^{16}$ times higher than that of a conventional wide bandgap material with optical features in the visible region.

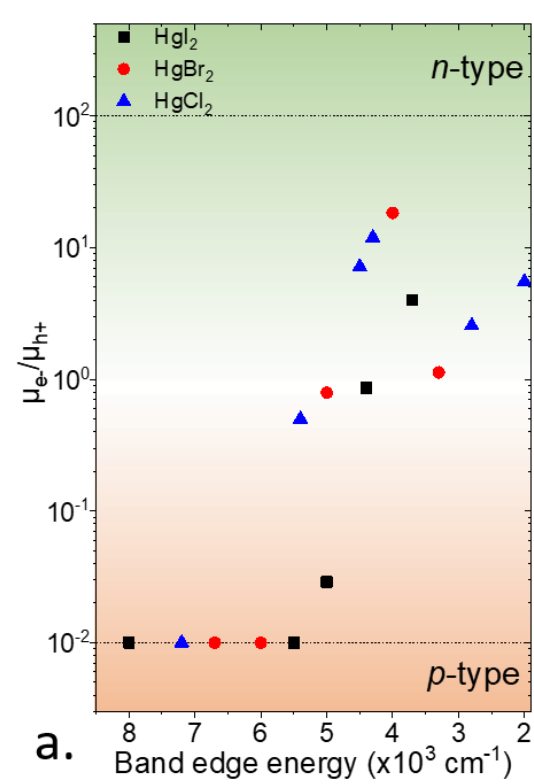

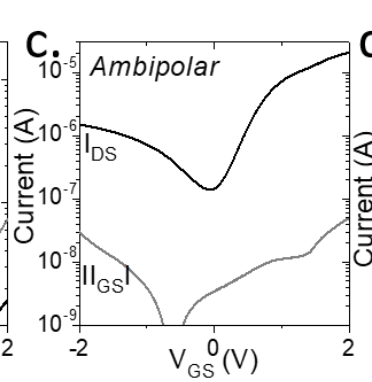
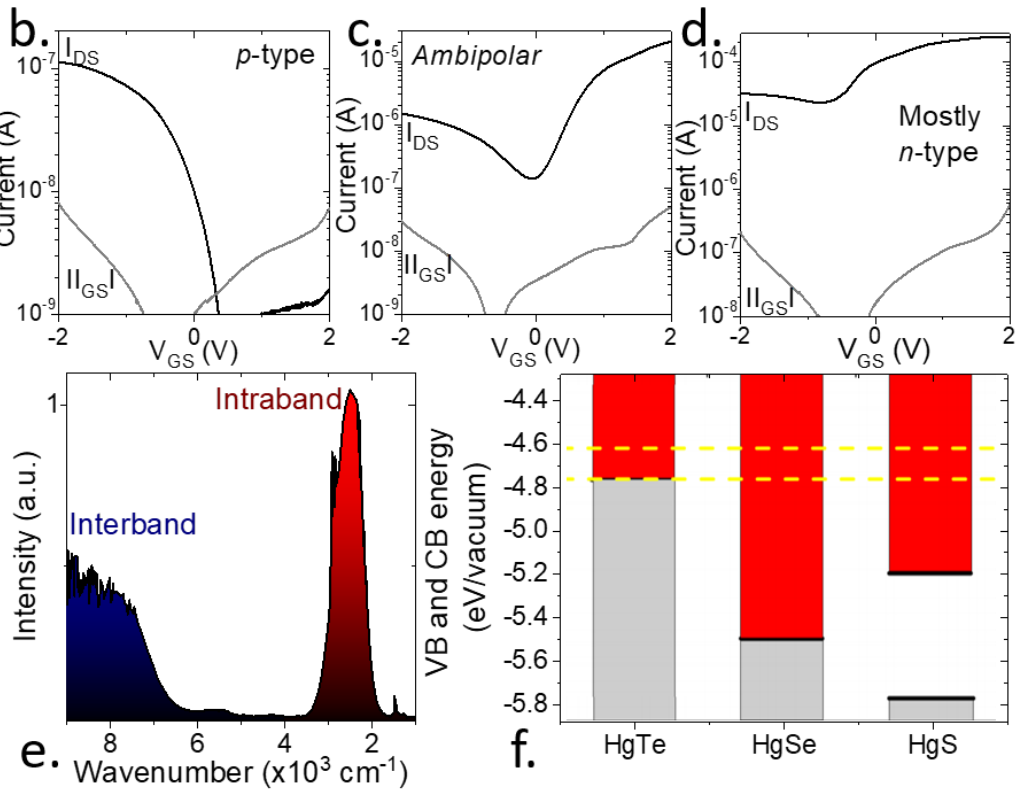
Figure 12 a. Ratio of the electron and hole mobilities $\left(\mu_{e}-\mu_{h_{+}}\right)$for thin films of $\mathrm{HgTe}$ with different band edges. When the material presents only p-type conduction, the ratio $\mu_{e-} / \mu_{h_{+}}$is set as $10^{-2}$. b., c. and d Transfer curves for electrolyte-gated thin films of $\mathrm{HgTe} C Q D s$ with band edges at $7000 \mathrm{~cm}^{-1}, 4000 \mathrm{~cm}$

1 and $700 \mathrm{~cm}^{-1}$, respectively. Parts a-d are adapted with permission from ref ${ }^{192}$. Copyright (2018) American Chemical Society. e Infrared spectrum of HgSe NCs. f. Energies of the valence band (gray volume) and conduction band (red volume) for $\mathrm{HgTe}, \mathrm{HgSe}$, and $\mathrm{HgS}$. The yellow dashed line shows the range of the Fermi level measured through electrochemical approaches. Part $f$ is reprinted with permission from ref ${ }^{68}$ Copyright (2017) American Chemical Society.

In addition, thermal activation originates from material nonstoichiometry. When using various synthetic procedures and precursors, ${ }^{30,96,192}$ small HgTe NCs present a p-type characteristic; see the transistor measurement exhibiting only hole conduction in Figure 12b. When the size is increased, a switch toward ambipolar conduction occurs, and finally, n-type characteristics occur for the largest particles (see Figure $12 \mathrm{c}$ and d). The threshold of this transition (see Figure 12a) depends on the growth process. ${ }^{96}$ The exact mechanism of this transition remains unclear; however, the mechanism can likely be correlated with the change in shape observed for HgTe with size. The difference in the exposed facets might induce a difference in the change in the amount of excess $\mathrm{Hg}$ cation surface with the particle size.

In HgX NCs, a second doping process occurs that leads to degenerate doping. This process is defined as self doping because high carrier densities (> 1 carrier per NC) can be achieved without introducing external impurities. Self doping is a specific behavior limited to narrow bandgap materials. Self doping plays a key role because it leads to intraband absorption (Figure 12e) in the steady state. ${ }^{65,66}$ This phenomenon allows the design of IR detectors for wavelengths larger than $3 \mu \mathrm{m} .{ }^{34,47,120}$ The intraband absorption signature is a relatively narrow peak, typically in the midinfrared region (see Figure 12e). This peak results from the optical transition within the conduction band between the $1 \mathrm{~S}_{\mathrm{e}}$ and $1 \mathrm{Pe}_{\mathrm{e}}$ states and was first observed for HgS NCs. ${ }^{33,193,194} \mathrm{This}$ intraband absorption, which is the signature of degenerate doping, was likely present in even older syntheses of HgS and HgSe NCs but was missed by researchers, who focused on visible and near IR absorption. HgSe exhibits a similar intraband absorption. ${ }^{29,34,118,124}$ Such intraband absorption was also later reported for $\mathrm{HgTe} ;^{30,61,63,96}$ however, fairly large HgTe NCs are required to observe this effect (> $10 \mathrm{~nm}$ typically). The band alignment of the bulk $\mathrm{HgX}$ material, observed through electrochemical approaches, ${ }^{68}$ as shown in Figure $12 \mathrm{f}$, allows the rationalization of these observations. $\mathrm{HgX}$ exhibits a fairly large work function (> $5 \mathrm{eV}$ for the bulk material), with a value of $4.6 \pm 0.1 \mathrm{eV}$, as determined through electrochemistry- and photoemission-based methods for short thiol-capped HgX NCs. ${ }^{49,122,192}$ Consequently, the bottom of the conduction band in this material is below the Fermi level. Thus, even in the presence of quantum confinement, the $1 \mathrm{~S}_{\mathrm{e}}$ state can be found below the Fermi level, and it becomes populated. The stable form of the QDs is negatively charged. In this sense, self doping can be considered to be a reduction of the NCs by their environment. ${ }^{186}$ The large band offset between HgSe and HgTe can explain the difference in behavior between the two materials. The conduction band of HgSe lies comparatively lower than the Fermi level, and only strongly confined HgSe NCs (i.e., size less than $3 \mathrm{~nm}$ ) exhibit the $1 \mathrm{~S}_{\mathrm{e}}$ state above the Fermi level (i.e., no intraband absorption feature exists). For HgTe, the conduction band is relatively close to the Fermi level, and thus, only the weakly confined particles can exhibit intraband absorption. HgS NCs exhibit an intermediate behavior because of the presence of a nonzero bulk bandgap.

It was clarified that this inherent doping could be used to control and tune the magnitude of the carrier density postsynthesis. Similar to what has been observed for PbS nanocrystals, ${ }^{195,196}$ the NC surface chemistry affects not only the internanoparticle coupling but also the electronic spectrum in terms of the dipole $\mu$ associated with the surface ligands, leading to a surface gating effect. The induced band bending is defined as $\Delta E_{v a c}=-N \frac{\mu_{\perp}(\text { ligand })}{\varepsilon_{0} \varepsilon_{\text {ligand }}}$, where $N$ is the surface density of the dipoles and $\varepsilon_{\text {ligand }}$ is the dielectric constant of the ligands. This induced shift is not related to the bandgap of the nanocrystal. Consequently, the induced relative ligand shift of the Fermi level with respect to the bands is considerably more dramatic for narrow bandgap materials. A series of ligands compatible with transport (i.e., typically short thiol based ligands that strongly bond to the surface $\mathrm{Hg}$ ) were investigated for HgTe and HgSe (see Figure 23c and Figure 13a-b, respectively). For small HgTe NCs (which have a band edge energy of approximately $720 \mathrm{meV}$ ), as depicted in Figure 23c, ligand exchange allows the tuning of the Fermi level energy within the bandgap of the material. Furthermore, this shift is sufficiently strong to induce a change in the majority carriers. Amine and thiocyanate lead to $n$-type NCs, while ethanedithiol or chloride lead to $p$-type NCs. For HgSe, as shown in Figure $13 a$, the effect is even more dramatic. While the absorption spectrum of $\mathrm{PbS}^{195}$ or $\mathrm{HgTe}^{49}$ is not considerably influenced by the ligand exchange process (generally, only a small redshift and band edge broadening are observed), the optical properties of $\mathrm{HgSe}$ are notably affected. ${ }^{186} \mathrm{HgSe} \mathrm{NC}$ absorption is strongly modulated as the Fermi level is displaced from within the conduction band to the bandgap. In this context, ligand exchange is a successful strategy to tune the doping level, typically over one order of magnitude, as shown in Figure 13b, by using only commercially available ligands that are compatible with charge transport. 
However, the dipole-induced tunability of the ligands is inadequate. To overcome this issue, Martinez et al. proposed using azobenzene (AZB) as a ligand. ${ }^{197}$ AZB is a small organic molecule with two conformations (cis and trans) of the aromatic group around the $\mathrm{N}=\mathrm{N}$ bond. ${ }^{198-200}$ Under UV and visible light illumination, the molecule can switch its conformation (from trans to cis, and vice versa, under UV light and visible light or heating, respectively). This change in conformation is commonly implemented to design molecular motors. ${ }^{201,202}$ Instead of the mechanical aspect, Martinez et al. exploited the change in dipole resulting from the conformation shift. Typically, the dipole of the cis configuration is larger than that of the trans conformation. ${ }^{203}$ To graft AZBs onto $\mathrm{HgX} \mathrm{NCs,} \mathrm{a} \mathrm{short} \mathrm{thiol} \mathrm{chain} \mathrm{was} \mathrm{added,} \mathrm{whose} \mathrm{role} \mathrm{was} \mathrm{to} \mathrm{bond} \mathrm{to} \mathrm{the} \mathrm{Hg} \mathrm{surface,} \mathrm{as} \mathrm{shown} \mathrm{in} \mathrm{the} \mathrm{inset} \mathrm{of} \mathrm{Figure} \mathrm{13c.} \mathrm{Using}$ this approach, the authors demonstrated that the dark conduction and photoresponse magnitude in the IR range can be controlled via UV and blue light stimulation (see Figure 13c). ${ }^{197}$ Note that despite the length of the thiol-functionalized AZBs, the HgX NC film functionalized by AZB is still conductive.

To further change the carrier density of the HgX NCs, Martinez et al. ${ }^{204}$ proposed grafting redox groups onto the surface. In practice, the authors used a highly oxidized form of tungsten stabilized in a polyoxometalate (POM). POMs are strong electron attractors, and by coupling these attractors to electron-rich NCs, a redox reaction occurs between the two groups. The authors used the same strategy as that for AZBs to graft the POMs onto the NCs, ${ }^{205}$ functionalizing the POMs with a short thiol chain used as a linker, ${ }^{204}$ as shown in Figure 13d. Electron transfer from the HgX NCs to the POMs was evidenced by the reduction of the intraband absorption of the NCs, and the reduced form of W (i.e., with an oxidation state lower than +VI) was detected through photoemission measurements. POMs are strong electron attractors, and up to 5 electrons per particle can be transferred with POM functionalization.

To ensure the completeness of the discussion on doping, although very few researchers have focused on the introduction of external impurities in the case of $\mathrm{HgX} \mathrm{NCs,} \mathrm{it} \mathrm{must} \mathrm{be} \mathrm{mentioned} \mathrm{that} \mathrm{Liu} \mathrm{et} \mathrm{al.} \mathrm{demonstrated} \mathrm{Mn} \mathrm{doping} \mathrm{of} \mathrm{HgS} \mathrm{nanoparticles.}{ }^{206}$ The authors introduced $\mathrm{Mn}$ (up to 15\%) into the $\mathrm{HgS}$ matrix and observed an enhanced magnetoresistance with a higher $\mathrm{Mn}$ content.
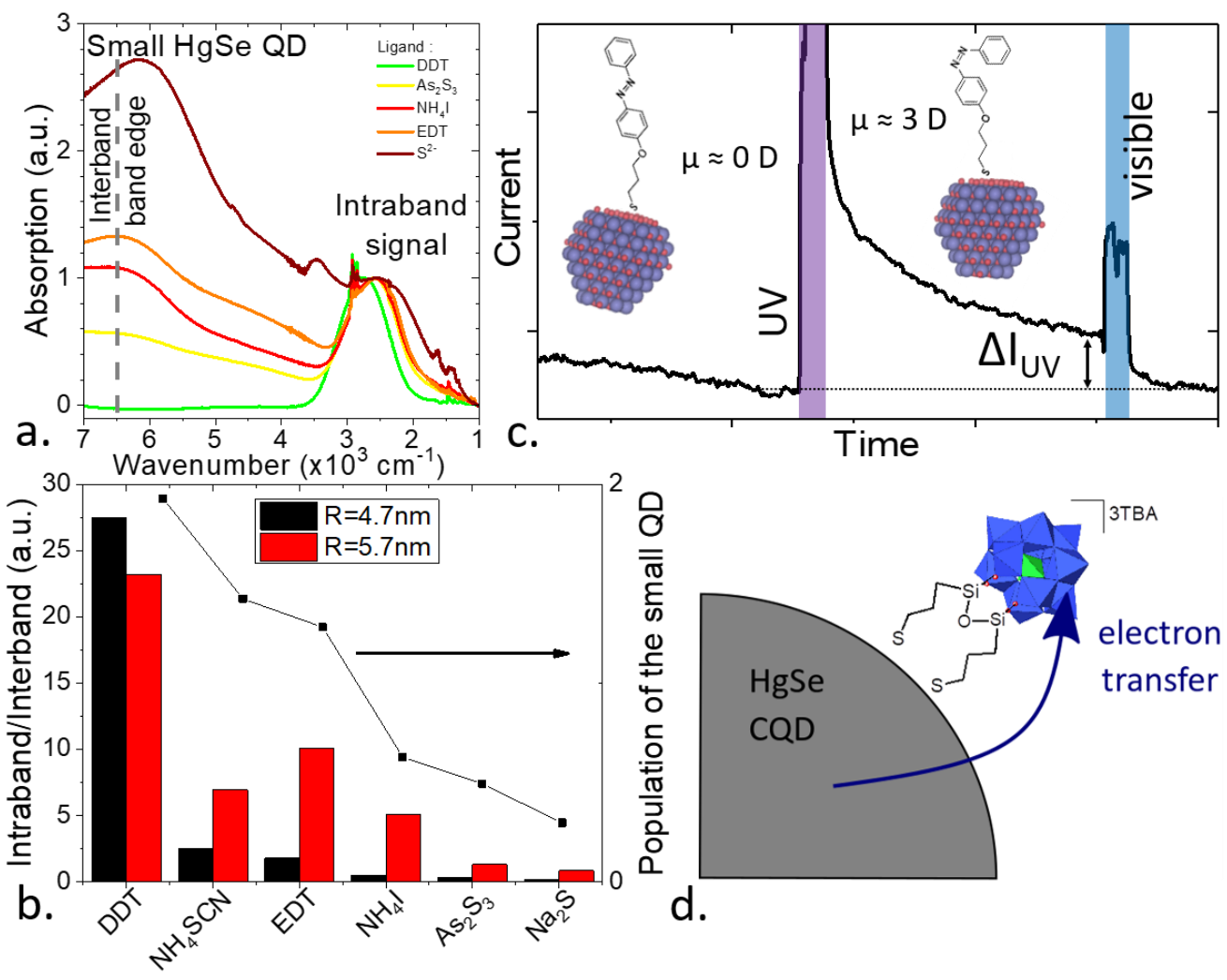

Figure 13. a. Absorption spectra of small $(4.7 \mathrm{~nm}) \mathrm{HgSe}$ nanocrystals capped with different ligands. $b$. Plot of the ratio of the intraband signal divided by the fitted magnitude of the interband signal for two sizes of HgSe nanocrystals. The related population of the CQDs is given on the right scale for small CQDs (diameter of $4.7 \mathrm{~nm}$ ). Graphs $a$ and $b$ are adapted with permission from ref ${ }^{186}$. Copyright (2016) American Chemical Society. c. Current in an azobenzene-capped HgTe film as a function of time. UV $(\lambda=365 \mathrm{~nm})$ or blue light $(\lambda=460 \mathrm{~nm})$ is projected on the film during the intervals highlighted with purple and blue rectangles, respectively. $\Delta l_{u v}$ is the change in the current as the azobenzene switches the configuration. Part $c$ is adapted with permission from ref ${ }^{197}$. Copyright (2018) American Chemical Society. d. Scheme of HgSe capped with POM-SH ligands. The arrows correspond to the electron 
transfer from doped $\mathrm{HgSe}$ QDs to the electron attractor POM-SH ligand. Part $d$ is adapted with permission from ref ${ }^{204}$ Copyright (2019), American Chemical Society.

Despite the notable progress that has been made to enhance the knowledge of doping and its tunability, the amount of work dedicated to doping in $\mathrm{HgX} \mathrm{NCs} \mathrm{remains} \mathrm{inadequate.} \mathrm{This} \mathrm{lack} \mathrm{of} \mathrm{information} \mathrm{is} \mathrm{detrimental} \mathrm{for} \mathrm{the} \mathrm{design} \mathrm{of} \mathrm{devices} \mathrm{with} \mathrm{a} \mathrm{vertical}$ geometry. Better control of the carrier density can facilitate the design of $p-n$ junctions with higher built-in electric fields and enhanced charge extraction. Diverse routes can be explored, including the introduction of impurities with $+\mathrm{I}$ or $+\mathrm{III}$ redox states within the HgX atomic lattice. The introduction of such impurities might be eased by the recent development of liquid $\mathrm{Hg}$-based synthesis ${ }^{96}$ from which many metals can easily be solubilized. In addition, doping at the NC array level through the introduction of donor and acceptor dopant nanoparticles is a promising approach and has already led to several successes ${ }^{207,208}$ in the case of lead chalcogenides. This pathway might be worth investigating in the case of HgX NCs. 


\section{ELECTRONIC STRUCTURE AND OPTICAL PROPERTIES}

The electronic structure of HgX NCs is unique and responsible for the many attractive properties of these materials. The spectral tunability of HgTe (Figure 2a), without a competing example, is directly connected to the semimetal nature of the bulk material. While this unique electronic structure leads to several opportunities, it is associated with several challenges. In the case of IV and III-V semiconductor groups, considerable research has been dedicated to the study of the electronic structure of the materials, and the associated results are highly accurate. This aspect is not true for II-VI semiconductors, for which, beyond the bandgap value, large discrepancies in the parameters describing the electronic structure can be observed in different studies. The state of $\mathrm{HgTe}$ is not the most inferior owing to the effort dedicated to its integration in IR sensors and topological insulator designs. Nevertheless, current knowledge on $\mathrm{HgX}$ materials is incomplete. This statement is more relevant when the material is in the nanocrystal form. In addition to the unique bulk properties, quantum confinement and surface chemistry affect the electrical spectrum as well. This aspect prevents the textbook design of colloidal heterostructures and devices, including films of HgX NCs. Despite these difficulties, substantial progress has been made over the past decades owing to the combination of various theoretical (k.p., DFT, tight binding) and experimental methods.

This chapter is organized as follows. We first discuss the electronic spectrum of bulk HgX materials and their associated characteristics. Next, we describe the confinement and systematically discuss the efforts dedicated to model the electronic structure of HgX NCs. A brief section is focused on the optical properties of HgX NCs. Subsequently, we describe the effects of temperature and pressure on the spectroscopic properties of HgX NCs. A section is also dedicated to the experimental determination of the absolute band alignment of $\mathrm{HgX} \mathrm{NCs,} \mathrm{considering} \mathrm{the} \mathrm{design} \mathrm{of} \mathrm{heterostructures} \mathrm{and} \mathrm{devices.} \mathrm{Finally,} \mathrm{the} \mathrm{last}$ section is dedicated to the dynamic aspects of the electronic structure, and we review the experimental methods used to examine carrier relaxation.

\subsection{Bulk material and confinement}

\subsubsection{From bulk HgX to NCs}

Conventional wide bandgap II-VI semiconductors, such as CdSe, exhibit a band gap in the visible region. The valence band states ( $\Gamma_{8}$ band) are mostly composed of the $p$ orbitals from the chalcogen. This band is strongly degenerate (eightfold) and split into light and heavy hole bands far from the $\Gamma$ point. The valence band exhibits weak dispersion, corresponding to a large hole effective mass. The conduction band ( $\Gamma_{6}$ band) is generally composed of the $s$ orbital of the metal. This band is twofold degenerate and is more strongly dispersive than the valence band. Specifically, the associated effective mass is lower. The spin orbit band is located in the valence band and is responsible for increasing the splitting between the light and heavy hole bands. A general trend is an increase in the spin-orbit coupling as the mass of the chalcogenides increases.

While switching from Cd-based to Hg-based II-VI materials, the band ordering is considerably affected (see Figure 14). HgTe is the most investigated material among the series of $\mathrm{HgX}$ compounds because of its use for IR sensing. This material exhibits an inverted band ordering, as observed for gray tin. ${ }^{209,210}$ The band with $\Gamma_{6}$ symmetry appears below the $\Gamma_{8}$ bands (see Figure $14 \mathrm{c}$ ). This inversion is generally formulated considering a negative bandgap value that describes the relative position of the $\Gamma_{6}$ and $\Gamma_{8}$ bands. This value corresponds to a $\Gamma_{6}$ value below the Fermi level. In HgTe, this negative bandgap is typically approximately -0.3 eV at 0 $\mathrm{K},{ }^{209}$ and it is reduced by approximately $-0.15 \mathrm{eV}$ at room temperature. ${ }^{72}$ Due to the band ordering inversion, the conduction band in HgTe corresponds to the band with light hole symmetry in cadmium chalcogenides. This band is strongly dispersive and associated with a weak electron effective mass (typically $0.01-0.03 \mathrm{mo}$ ). ${ }^{62,72}$ This low value of the effective mass leads to the large carrier mobility ( $>1000 \mathrm{~cm}^{2} \mathrm{~V}^{-1} \mathrm{~s}^{-1}$ and $>10^{5} \mathrm{~cm}^{2} \mathrm{~V}^{-1} \mathrm{~s}^{-1}$ at room temperature and low temperature ${ }^{211}$, respectively) observed in bulk HgTe. In contrast, the valence band is almost flat, and the heavy hole mass is larger than $0.3 \mathrm{~m}_{0} .{ }^{72}$ The Fermi level for the intrinsic HgTe lies between these two bands, which share the same energy at the $\Gamma$ point. This aspect is the second key characteristic of the bulk HgTe band structure: the degeneracy of the valence and conduction bands at the $\Gamma$ point causes HgTe to exhibit zero bandgap semiconductor characteristics, corresponding to a semimetal. This lack of a bulk bandgap is the reason for the ultrabroad tunability of the optical spectrum of HgTe NCs (see Figure 2a) compared to that of the other semiconductors, for which the spectral tunability is limited by the material bulk bandgap. In HgTe, the band edge energies can be designed to be arbitrarily narrow, thereby expanding the absorption of this material toward extremely large wavelengths in the infrared region. The optical absorption in HgTe occurs between the less dispersive valence band and conduction band. In this sense, the optical bandgap is always positive and should not be erroneously considered as the negative value mentioned previously, which only describes the band ordering. At the $\Gamma$ point, the two bands with $\Gamma_{8}$ symmetry are mostly composed of $p$ Te orbitals. Nevertheless, the strong band mixing in $\mathrm{HgTe}$ causes the wave functions to acquire the $s$ nature from the $\mathrm{Hg}$ atoms as soon as the wave vector is moved far (few $\%$ of $\pi / R$ ) from the $\Gamma$ point. 

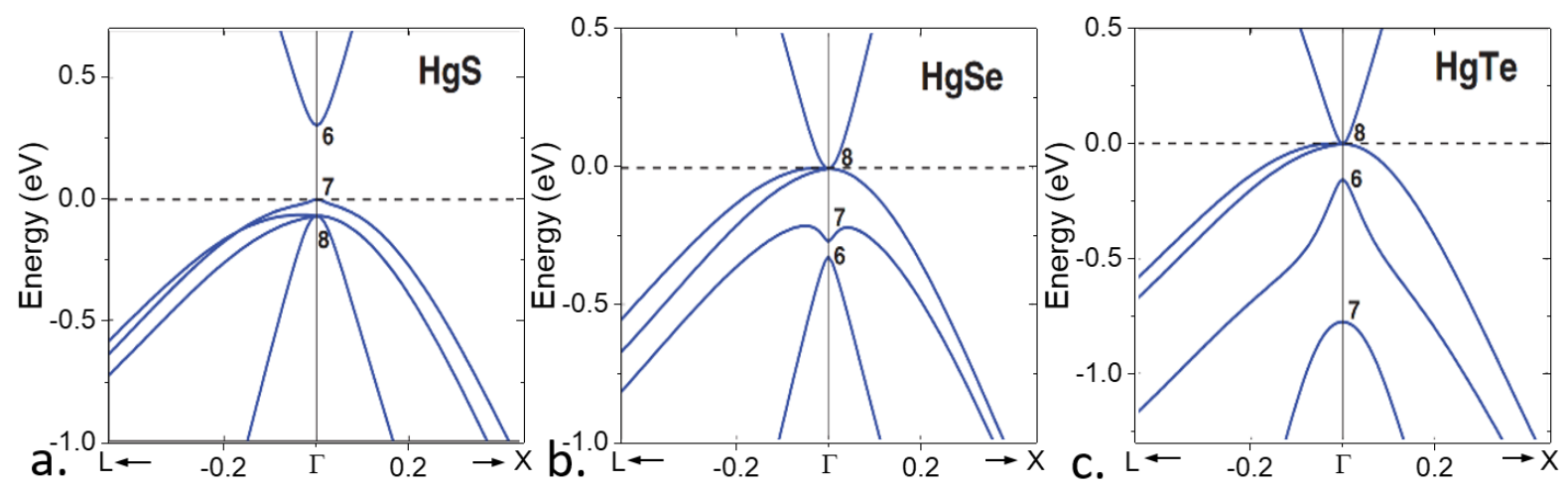

Figure 14 a. Band structure of $\mathrm{HgS}$ near the $\Gamma$ point from a hybrid QSGW approach. The unit along the $x$ axis is $2 \pi / a$, with $a=0.584 \mathrm{~nm}$. $b$. Band structure of HgSe near the $\Gamma$ point from a hybrid QSGW approach. The unit along the $x$ axis is $2 \pi / a$, with $a=0.608 \mathrm{~nm}$. c. Band structure of $\mathrm{HgTe}$ near the $\Gamma$ point from a hybrid QSGW approach. The unit along the $x$ axis is $2 \pi / a$, with $a=0.647 \mathrm{~nm}$. Reprinted with permission from ref ${ }^{70}$ Copyright (2011) by the American Physical Society.

$\mathrm{HgSe}$ presents a band structure similar to that of $\mathrm{HgTe}$, although, quantitatively, the negative bandgap value is lower. HgSe can also be described as a zero bandgap semiconductor with an inverted band ordering. Nevertheless, the lower mass of Se compared to Te reduces the spin-orbit coupling, and the $\Gamma_{7}$ band lies above the $\Gamma_{6}$ band (see Figure $14 \mathrm{~b}$ ). Among $\mathrm{HgX}$ materials, $\mathrm{HgS}$ appears to be an exception as a narrow bandgap semiconductor. ${ }^{212}$ The exact value of the bandgap is typically a few hundred meV: ${ }^{68,70,213}$ a value of approximately $0.65 \mathrm{eV}$ matches that reported in spectroscopy experiments with HgS NCs. ${ }^{68}$

Once HgX materials are grown in the NC form, quantum confinement occurs, leading to the following two phenomena. First, the NCs are OD objects, and the density of the states becomes discrete. Second, the finite size of the particles makes the center part of the Brillouin zone (i.e., close to the $\Gamma$ point) no longer accessible, and the band edge energy increases (i.e., a bandgap opens). In HgTe and HgSe, due to the lack of a bulk bandgap, the NC band edge energy is purely due to confinement, as shown in Figure 12f. Due to the strong asymmetry of the hole and electron effective masses $\left(\mathrm{m}_{\mathrm{h}}^{*} / \mathrm{me}_{\mathrm{e}}^{*}>10\right)$, confinement mostly occurs in the conduction band, owing to which the density of the states in the conduction band is lower than that of the states in the valence band.

\subsubsection{Effective mass approximation and multiband k.p model}

The effective mass approximation (or two band k.p model) is the simplest strategy to model the electronic structure of the NC and accounts for the confinement. As a primitive approach, confinement is considered through the evaluation of the wave vector $k=\pi / R$, where $R$ is the particle radius. Next, the energy of this wave vector is evaluated. In this simple model, two assumptions are considered. First, spherical particles are considered, which is often not the case for HgTe; see Figure 5a or Figure 6c. Particles with other shapes may present a different relation between $k$ and the particle size, which is likely not analytic. Second, infinite confinement is considered, which, a posteriori, is a reasonable approximation. Such models, have been used to estimate the sizing curve (energy of the optical transition vs. size), ${ }^{93,114}$ as shown in Figure 15 a.

Owing to the ease of parametrization, the effective mass approximation can easily be applied to heterostructures. The time independent Schrodinger equation in a spherical geometry can be expressed as $\left[-\frac{\hbar^{2}}{2 m^{*}}\left(\frac{2}{r} \frac{\partial}{\partial r}+\frac{\partial^{2}}{\partial r^{2}}\right)+V(r)\right] \psi(r)=E \psi(r)$, where $\hbar$ is the reduced Planck constant, $m^{*}(r)$ is the effective mass profile, $V(r)$ is the energy profile for the valence and conduction bands and $\psi(r)$ is the wave function. The material parameters are injected through the mass and energy profiles (bandgap and band alignment) of the bulk. This method is typically used to observe the wave function localization in heterostructures. Moreover, this approach has been applied to the spherical quantum well obtained from the CdS/HgS(/CdS) heterostructure, ${ }^{125}$ as shown in Figure $15 \mathrm{c}$ and $\mathrm{d}$, to observe the electron and hole localizations within HgS resulting from a type I band alignment between the two materials. A similar approach has been applied to HgSe/HgTe heterostructures, ${ }^{120}$ as shown in Figure $15 \mathrm{e}$ and $\mathrm{f}$. In this case, the two bulk materials exhibit a type II band alignment according to Figure $12 \mathrm{f}$. As a HgTe shell is grown around a HgSe core, the electron wave function remains localized in HgSe. Consequently, the intraband transition resulting from the doping of HgSe is not considerably affected by the presence of the HgTe shell, as shown in Figure 15e. In contrast, the hole wave function is promptly localized in the $\mathrm{HgTe}$ shell as it is grown, as shown in Figure $15 f$. 
However, the validity of the effective mass approximation is extremely limited. The 14-band k.p model indicates, as shown in Figure 15b, that the bands in HgTe are only parabolic in a small portion of the Brillouin zone, typically less than $1 \%$. Consequently, the sizing curves based on the effective mass approximation are only valid for a small range of sizes; see Figure 15a.
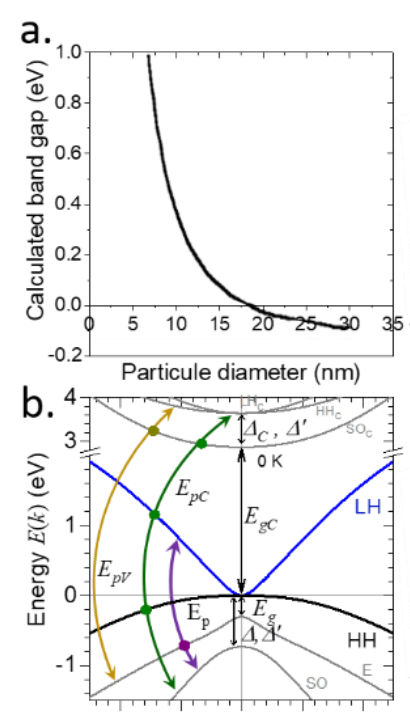

$\begin{array}{lllll}-0.2 & -0.1 & 0.0 & 0.1 & 0.2\end{array}$

Wave vector $k(2 \pi / a)$ along $\Gamma-\mathrm{X}$
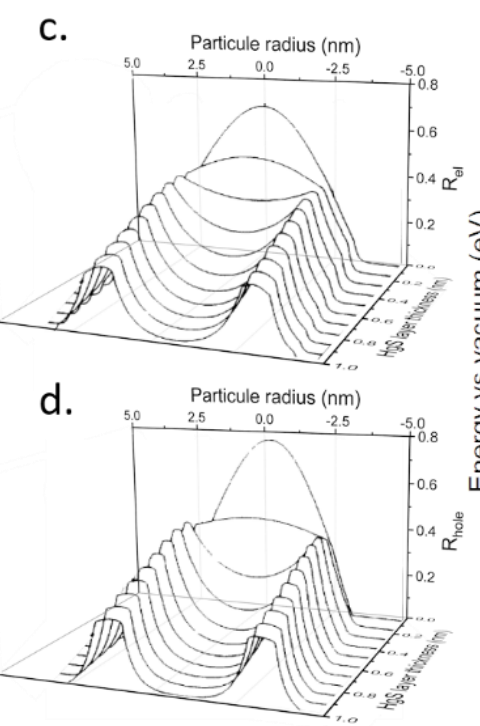

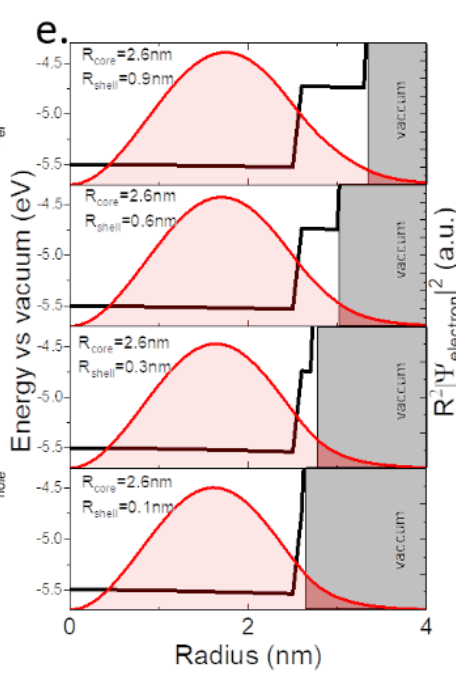

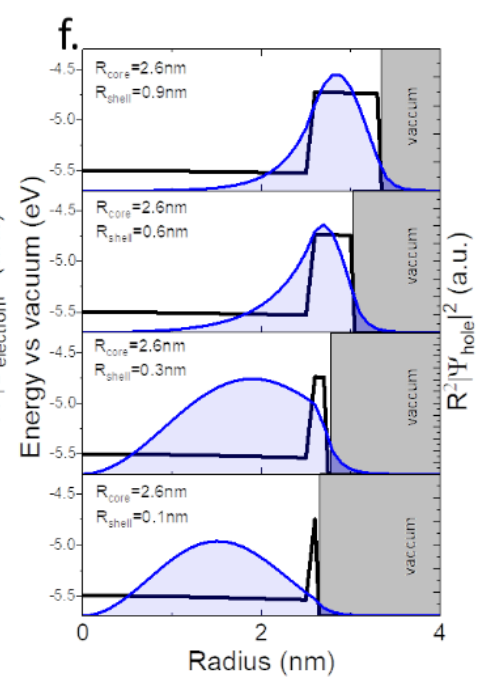

Figure 15 a. Prediction of the bandgap from the HgTe particle size using the effective mass approximation. Part a is used with permission from the Royal Society of Chemistry, from ref ${ }^{93}$; permission conveyed through the Copyright Clearance Center. b. Simulated dispersion $E_{i}(k)$ for the 14 bands of the k.p model. Part $b$ is adapted with permission from ref ${ }^{62}$ Copyright (2020) American Chemical Society. Radial wave functions of the electrons (c.) and holes (d.) in CdS/HgS/CdS heterostructures with various $\mathrm{HgS}$ thicknesses. Parts $c$ and $d$ are reprinted with permission from ref ${ }^{125}$ Copyright (1994) by the American Physical Society. Conduction band (e.) and valence band profiles (f) and electron/hole density distribution in $\mathrm{HgSe} / \mathrm{HgTe}$ heterostructures for various thicknesses of the HgTe shell. Parts e and $f$ are adapted with permission from ref ${ }^{120}$ Copyright (2018) American Chemical Society.

When the description of the electronic structure requires the consideration of a broader range of energy, more bands need to be included. A recommendation is to use a 2+1-band model, ${ }^{114,193}$ in which the light hole and conduction bands are described by a two-band k.p model and coupled through a Kane parameter, while the heavy hole band is treated as being uncoupled due to its large effective mass. The spin-orbit band, approximately $1 \mathrm{eV}$ below the heavy hole band, is neglected. When a detailed description of the valence band is required, an 8 band k.p model describing the $\Gamma_{6}, \Gamma_{7}$ and $\Gamma_{8}$ bands and their degeneracies is used. ${ }^{72,214}$ It has been estimated that the 8-band k.p model correctly describes the first $8 \%$ of the Brillouin zone. ${ }^{72}$ Although the effective mass approximation only describes the bottom parabolic part of the light hole $\left(\Gamma_{8}\right)$ band, the inclusion of an additional valence band is critical to capture the linear dispersion occurring at higher wave vectors.

When highly confined forms of HgTe are considered, as in the case for NPLs, it is necessary to account for higher conduction bands (Figure 15b) since the conduction band is responsible for most of the confinement due to the mass asymmetry. In this case, an accurate description of the bands close to the Fermi level but away from the $\Gamma$ point can be obtained using the 14 band k.p approach. ${ }^{62}$ The inclusion of 14 bands correctly describes $\approx 15 \%$ of the Brillouin zone. ${ }^{62}$ The additional upper conduction bands are responsible for the deviation from linearity of the light hole band observed at large wave vectors (Figure 15b), which, as described later in this chapter, is responsible for the inversion of the temperature induced band edge shift with the particle size (see Figure 18). 

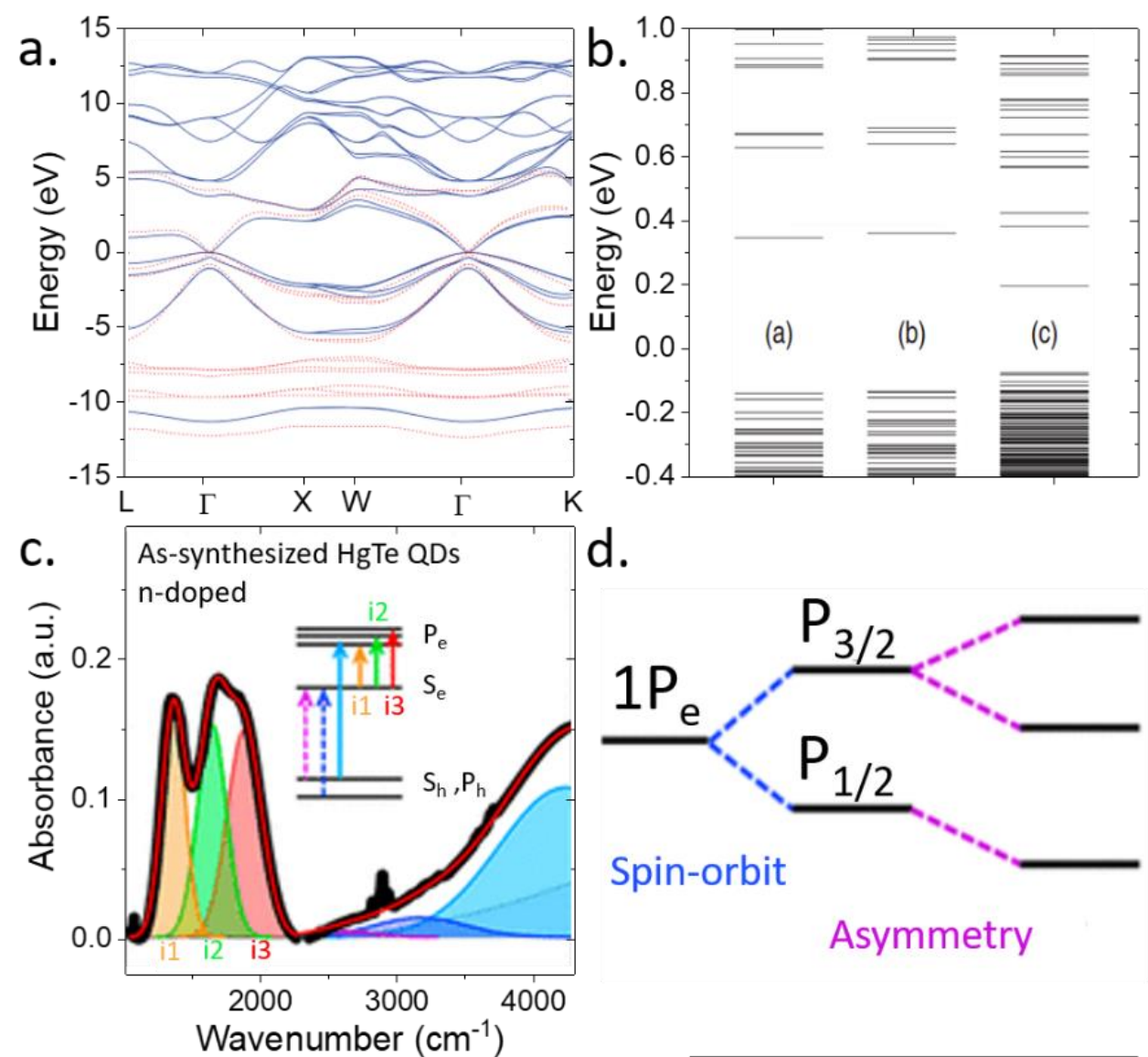

d.

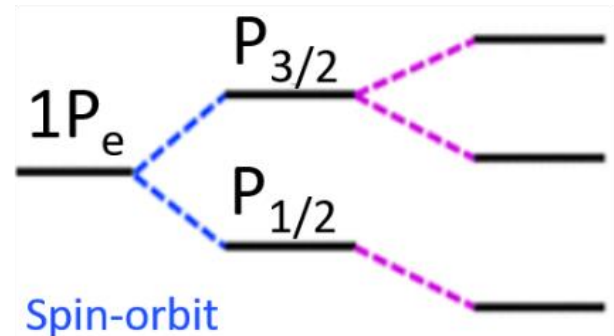

Spin-orbit

\section{Asymmetry}

e
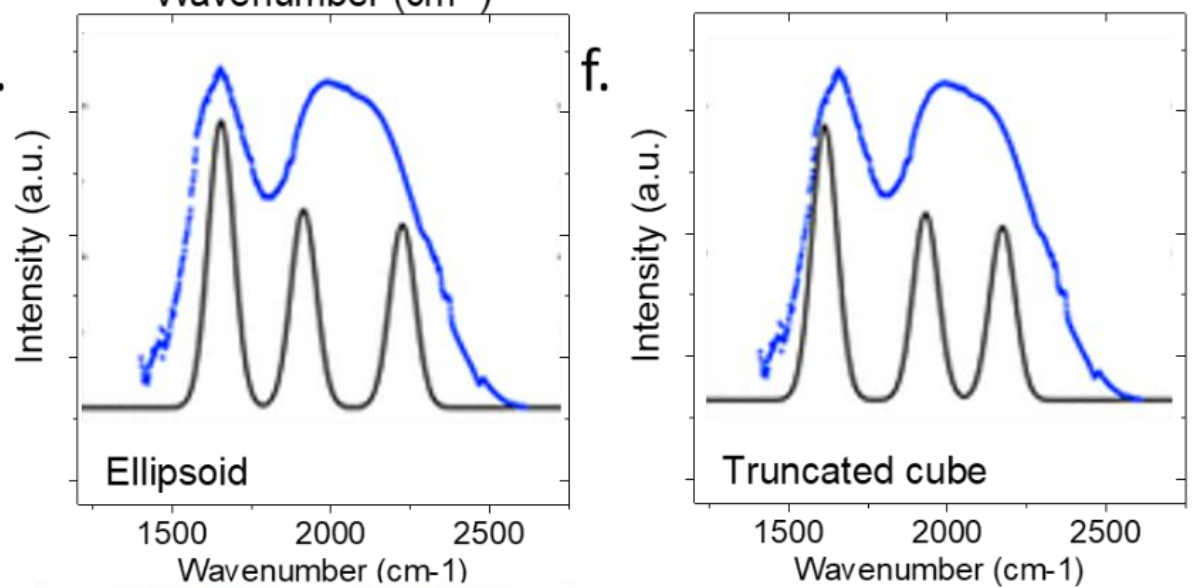

g.
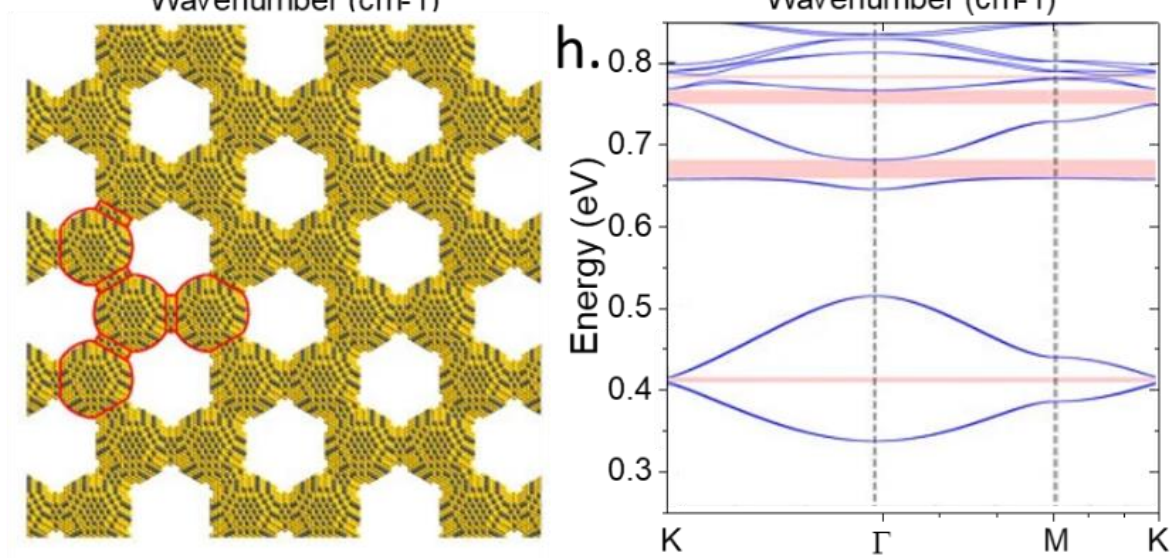

Figure 16 a. (Color online) Band structure of $\mathrm{HgTe}$ at $\mathrm{O} \mathrm{K}$ calculated in tight binding. The red dots indicate the values obtained using a hybrid GW approach. Zero energy corresponds to the top of the valence band. b. Calculated energy levels in HgTe QDs at $300 \mathrm{~K}$ [(a) sphere, $d=5.98 \mathrm{~nm}$; (b) octahedron, $d=5.83 \mathrm{~nm}$; (c) sphere, $d=8.99 \mathrm{~nm}$. Zero energy corresponds to the top of the bulk valence 
band. Parts $a$ and $b$ are reprinted figures with permission from ref ${ }^{216}$ Copyright (2012) by the American Physical Society. c. Absorption spectrum (black line=experiment) of large doped HgTe NCs. The red line is a fit obtained from a sum of Gaussians and indicates that doping suppresses the first two excitonic transitions and induces intraband absorbance $\left(i_{1}-i_{3}\right) . d$. Schematic demonstrating the splitting of the $P_{e}$ state by both spin-orbit coupling and structural asymmetry. e and $f$. Predicted intraband absorbance spectra (black lines) for several shapes of $\mathrm{HgTe} Q \mathrm{QD}$ with a spherical volume with a radius of $4 \mathrm{~nm}$, and comparison with experimental data for a solution of $7.6 \mathrm{~nm}$ diameter QDs (blue points). Parts $c-f$ are adapted with permission from ref ${ }^{61}$ Copyright (2018) American Chemical Society. g. Top view of an assembly of spheres connected by cylinders that form a honeycomb lattice of HgTe. The $\mathrm{Hg}$ and Te atoms are presented in yellow and gray, respectively. $h$. Conduction band dispersions resulting from the atomistic tight binding calculation. Nontrivial gaps are indicated by pink shaded regions. Parts $g$ and $h$ are adapted from ref ${ }^{215}$.

Tight binding methods are reasonably effective for describing HgTe NCs. The input parameters are less straightforward to determine compared to k.p modeling. However, the method is less demanding in terms of calculation than DFT, and it can be applied to larger NCs and even superlattices. ${ }^{215}$ Allan and Delerue used an $\mathrm{sp}^{3} \mathrm{~d}^{5} \mathrm{~s}$ ( basis to reproduce the electronic spectrum of bulk HgTe and nanocrystals (see Figure 16a and b). ${ }^{216}$ The authors observed that a deviation from the spherical form had a limited impact on the spectrum. Moreover, the authors concluded that coulombic corrections exerted only marginal contributions to the optical spectrum. Although the excitonic effects are weak at the band edge, HgTe remains an interesting platform to observe excitonic and multiexcitonic effects. The narrow bandgap nature of HgX NCs enables the realization of multiexcitonic effects at relatively low photon energies. Taking into account both energy and momentum conservation, the threshold voltage for the Auger multiplication effect ${ }^{217}$ to occur is $E_{t h}=\left(1+\frac{2+m_{c}^{*} / m_{h}^{*}}{1+m_{c}^{*} / m_{h}^{*}}\right) E_{G}$, where $E_{G}$ is the bandgap and $m_{c}^{*}$ and $m_{h}^{*}$ are the electron and hole effective masses, respectively. In HgTe, the strong effective mass asymmetry almost suppresses the excess energy due to the momentum conservation, leading to $E_{t h} \approx 2 E_{G}$. Experimentally, the threshold for multiexciton generation is slightly higher, approximately 3 times the bandgap, as obtained using both optical ${ }^{218}$ and transport measurements. ${ }^{219}$

Tight binding was later used to explain the linewidth of the intraband absorption of HgTe. ${ }^{61}$ In HgSe and HgS, the intraband transition appears as a single peak (Figure 12e); for $\mathrm{HgTe}$, the peak corresponds to more than one contributions (see Figure 16c). A naive interpretation may have been to attribute this second contribution to polydispersity. However, this effect is only observed with an enhancement of the monodispersity of $\mathrm{HgTe} \mathrm{NC}$ synthesis based on the $\mathrm{TMS}_{2}$ Te precursor. ${ }^{63,64}$ Tight binding simulations clarify that the large spin-orbit coupling in HgTe increases the degeneracy of the $1 \mathrm{P}_{\mathrm{e}}$ state (see Figure 16d). Because HgSe is lighter, the spin-orbit coupling is weaker, and thus, the $1 \mathrm{P}_{\mathrm{e}}$ state remains degenerate. A second lifting of the degeneracy also results from crystal field splitting (see Figure 16e and f). Contrary to the interband, which appears to be weakly influenced by the shape, 1Pe splitting depends on the exact nanoparticle shape. The power of tight binding modeling is more notable when more complex structures are considered. In 2013, Evers et al. reported connected superlattices of PbSe NCs, in which the NCs were necked and arranged into squares and honeycomb lattices. ${ }^{220-222}$ Such self assemblies provide an opportunity to merge the field of nanocrystal superlattices with that of superlattice transport. For the first time, it becomes possible to combine a high degree of organization with strong coupling, which may facilitate the design of high mobility films. ${ }^{223,224}$ Through development of this material, the growth of materials with a fractal dimensionality of 2-x can be examined, for which the electronic properties can be engineered by tuning both the composition and coupling. Beugeling et al. examined the electronic structure resulting from a similar ordered honeycomb lattice made of necked $\mathrm{HgTe} \mathrm{NCs}^{215}$ see Figure $16 \mathrm{~g}$. The direct synthesis of the structure is likely not be attributed to the HgTe NC solution since the necking process observed for PbSe benefits from the truncated cube shape due to the rock salt structure of PbSe. In addition, such a lattice may be obtained by cation exchange from PbTe preformed superlattices. This artificial graphene is especially promising since, contrary to its carbon based counterpart, it exhibits a large spin-orbit coupling, which is of considerable interest for spintronic applications. Beugeling et $a^{215}$ observed the formation of a Dirac cone and topological bandgaps as large as $30 \mathrm{meV}$ in such a superlattice (see Figure $16 \mathrm{~h}$ ). Although this work is theoretical, it likely paves the way toward more complex optoelectronic properties beyond the simple tunability of the bandgap with confinement. This work suggests that ordering NCs at the supracrystal level might be a promising strategy to achieve high carrier mobility in NC arrays.

\subsubsection{DFT calculations}

Density functional theory (DFT) remains marginally used to describe the electronic structure of HgX NCs. This lack of results mostly reflects the demanding computing power required for this method to be applied to large NCs. Geiregat et al. ${ }^{22}$ used DFT to clarify the origin of the low stimulated emission observed in small HgTe NCs; see the section dedicated to lasing and Figure 27a and b. 
The authors observed, in particular, long lived (several $\mu \mathrm{s}$ ) PL signals and correlated this observation to the surface trap states (Figure 17b) that can occur in undercoordinated HgTe NCs. DFT shows (Figure 17a) that the resulting gap states are close to the maximum valence band state. The presence of these states eases the population inversion in these NCs by forming a four level system (see Figure $27 \mathrm{~b}) .^{22}$

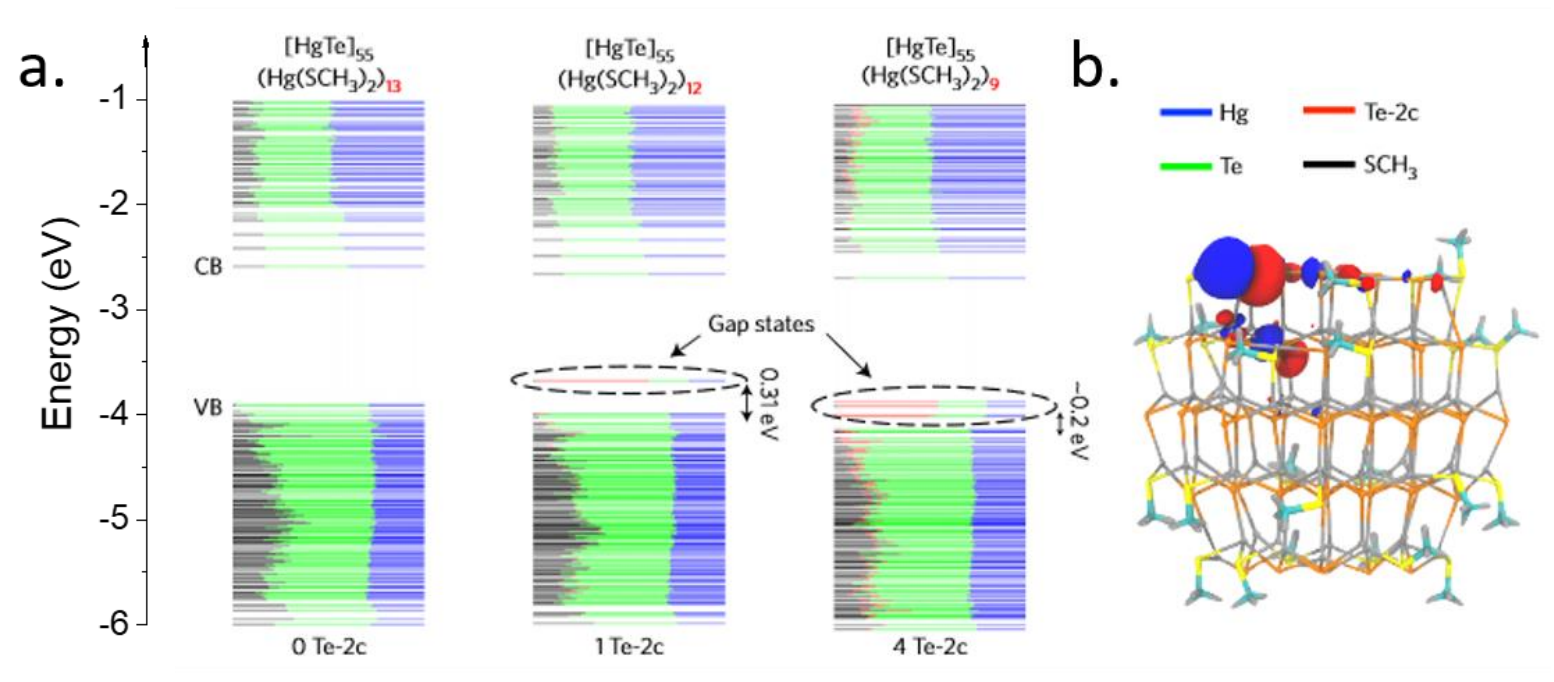

Figure 17 a. Energy level spectra of (left) a $[\mathrm{HgTe}]_{55}\left(\mathrm{Hg}\left(\mathrm{SCH}_{3}\right)_{2}\right)_{13}$ model nanocrystal; (center) the same nanocrystal after the removal of one $\mathrm{Hg}\left(\mathrm{SCH}_{3}\right)_{2}$ complex and (right) four $\mathrm{Hg}\left(\mathrm{SCH}_{3}\right)_{2}$ complexes, calculated using density functional theory. The colors indicate the projection of each state on (blue) $\mathrm{Hg}$ atoms, (green) Te atoms, (black) methanethiolate and (red) dicoordinated $\mathrm{Te}$ on different atom/fragment types. The successive removal of one $\mathrm{Hg}\left(\mathrm{SCH}_{3}\right)_{2}$ complex results in a band of gap states separated by approximately $100 \mathrm{meV}$ from the highest occupied molecular orbital (HOMO). $b$. Wave function of the gap state. This state largely corresponds to the $5 \mathrm{p}$ orbital of a two coordinated tellurium surface atom that is orthogonal to the neighboring $\mathrm{Hg} 6 \mathrm{~s}$ orbitals. Figure is reprinted with permission from Nature, Nature Materials, ref 22, [COPYRIGHT] (2018) 


\subsection{Optical properties}

One of the most striking properties of $\mathrm{HgX} \mathrm{NCs}$ is their widely tunable absorption (see Figure 2a). The first optical feature of HgTe NCs can be tuned from $11000 \mathrm{~cm}^{-1}(880 \mathrm{~nm}$ or $1.4 \mathrm{eV})$ to $150 \mathrm{~cm}^{-1}(60 \mu \mathrm{m}$ or $20 \mathrm{meV})$. The missing information is that the nature of the transition changes as well. The observed optical feature undergoes a change of origin, from interband (smallest NCs) to intraband ${ }^{29,33,65}$ and, finally, and possibly to plasmonic ${ }^{136}$ (for sizes larger than the Bohr radius) transitions. Note that this process is no longer limited to mercury chalcogenides and has been recently observed for PbS(e) NCs. ${ }^{225-227}$ Because the confinement drives absorption spectrum, the largest particles have the reddest absorption. The doping magnitude is also controlled by the confinement through the relative position of the conduction band with respect to the Fermi level, the highest doping is thus achieved for the largest particles. Following a Drude model, the free carrier density $n$ can be linked to the surface plasma frequency $\omega_{L S P R}$ through the simple expression $\omega_{L S P R} \alpha \sqrt{n}$. Thus, in conventionally doped semiconductors, higher doping leads to a blueshift of the plasmonic absorption. This phenomenon is typically observed for ITO nanocrystals (see bottom of Figure 2a) as the tin content increases in the $\ln _{2} \mathrm{O}_{3}$ matrix. In self-doped NCs, the opposite trend is observed and this stress the strong role of confinement in $\mathrm{HgX} \mathrm{NCs}$.

A key emerging direction is the control of light matter coupling within films of infrared NCs. As a film of NCs is coupled to a photonic structure, enhanced $\mathrm{PL}^{21}$ and absorption ${ }^{40,38}$ can be achieved. Using this approach, the spectral response can be shaped. The absorption spectrum is no longer driven by the material but can be strongly affected by its coupling to light resonators. More details regarding such devices with controlled light-matter coupling are presented in the chapter dedicated to light emission and photodetection. However, a common aspect in these approaches is the requirement for a high level of electromagnetic design. The inputs of these simulations are the geometrical parameters and complex optical indexes. To date, determination of the optical index in the infrared region has not been performed for $\mathrm{HgX} \mathrm{NC}$ films, which impedes the coupling of NC films to complex photonic structures. This lack of data relates to the low availability of infrared ellipsometers with operations above $1.7 \mu \mathrm{m}$. Bejaoui et al.

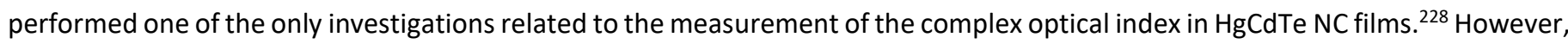
the NCs are small and include $\mathrm{Cd}$, and the band edge is not in the infrared region. Current simulations ${ }^{40}$ typically assume a constant real part for the NC optical index equal to 2. The real part of the dielectric constant of bulk HgTe at room temperature is approximately $\approx 7$, and this value increases to above 10 as cryogenic temperatures are reached. ${ }^{229,230} \mathrm{~A}$ value of 2 for the NC film is consequently lower than $\sqrt{\varepsilon}$, which accounts for the presence of remaining organics within the film. This value seems convenient for preliminary design; however, more accurate determination, including spectral dependence, is necessary.

In terms of absorption, the cross-section far from the band edge is a key tool to estimate the amount of crystalline material independent of the size and shape of the particles. For $\mathrm{HgTe}$, the cross-section at $415 \mathrm{~nm}$ is $\sigma_{415}(\mathrm{Hg})=2.6 \pm 0.4 \times 10^{-17} \mathrm{~cm}^{2} / \mathrm{Hg}$ atom. ${ }^{114}$ The absorption coefficient at the band edge is $3 \times 10^{3} \mathrm{~cm}^{-1}$ at $2.6 \mu \mathrm{m}\left(3900 \mathrm{~cm}^{-1}\right)$ and decreases as larger particles are grown. The value is estimated to be $1.5 \times 10^{3} \mathrm{~cm}^{-1}$ at $3.5 \mu \mathrm{m} .{ }^{114}$ This value is similar to the absorption observed for bulk $\mathrm{HgTe}{ }^{72} \mathrm{This}$ aspect is a major difference from epitaxially grown quantum dots. While epi-QD obtained considerable success in terms of quantum engineering ${ }^{231,232}$ and as single photon emitters, ${ }^{233,234}$ films of epitaxially grown quantum dots exhibit weak absorption due to their extremely low density ( $10^{11}$ QD. $\mathrm{cm}^{-2}$ per plane). Consequently, epitaxially grown quantum dots exhibit considerably weaker absorption than the bulk material. Using NCs, dense films can be fabricated (only limited by the NC packing), and thus, the absorption can be as large as that in bulk. Nevertheless, the absorption coefficient being below $10^{4} \mathrm{~cm}^{-1}$, several micrometers of film are required to absorb most of the incident light. At present, such thicknesses are one order of magnitude larger than the carrier diffusion length and remain difficult to achieve while maintaining high quality (i.e., smooth and crack free) films. This aspect is the reason for the criticality of the control of light-matter coupling; more details are presented in the section dedicated to this aspect in the last chapter.

In terms of the intraband transitions, the absorption coefficient is between 2 and $3 \times 10^{3} \mathrm{~cm}^{-1}$ for $5-6 \mathrm{~nm}$ large HgSe NCs, ${ }^{29}$ corresponding to intraband peaks in the 3-5 $\mu \mathrm{m}$ range. This large absorption is one of the benefits of intraband-based detectors compared to those relying on interband absorption.

\subsection{Temperature and pressure dependence}

The temperature and pressure dependence dramatically affect the spectral response of HgX NCs. The influence of temperature effects on the electronic spectrum is of interest since cryogenic operation is usually implemented for infrared devices. As the bandgap is affected by temperature, the cutoff wavelength can be modified. The effect of pressure can be considered as a nonconventional strategy to tune the NC spectrum. The motivation to examine the pressure dependence originates from the design of colloidal heterostructures, in which the growth of two nonmatched lattice semiconductors generates strain. An independent investigation of the pressure effect allows us to disentangle the changes in the electronic spectrum resulting from strain and that derived from charge delocalization. 


\subsubsection{Effect of temperature}

In conventional semiconductors ( $\mathrm{CdTe}, \mathrm{CdSe}$ ), the trend is to observe a redshift as the temperature increases. Bulk HgTe is an exception to this rule, exhibiting a positive value for $\mathrm{dE}_{\mathrm{G}} / \mathrm{dT} .{ }^{235-237} \mathrm{HgTe} \mathrm{NCs}$ present a similar trend, as reported by Lhuillier et al. ${ }^{114}$ However, HgTe NCs are not the only NCs that present such an inverted temperature dependence, as it has also been reported for lead chalcogenides ${ }^{238}(\mathrm{PbS})$ and, more recently, for lead halide perovskites ${ }^{239}\left(\mathrm{CsPbBr}_{3}\right)$. The first native effect of temperature is thermal dilatation. Upon heating, the lattice may expand to affect the particle size and thus the confinement. However, this effect appears to be quantitatively marginal. ${ }^{114,238}$

This temperature dependence depends on the size of the quantum dots. While HgTe NCs present a blueshift of the band edge upon heating (see Figure 18b), HgTe NPLs exhibit an inverted trend (see Figure 18a). Nevertheless, this sign inversion in $\mathrm{dE}_{\mathrm{G}} / \mathrm{dT}_{\mathrm{T}}$ is not driven by the shape of NPLs but by their confinement. In other words, NCs and NPLs exhibit similar $\mathrm{dE}_{\mathrm{G}} / \mathrm{dT}$ values for similar band edge energies, as shown in Figure $18 \mathrm{c}$ and $d$. As the confinement increases in $\mathrm{HgTe}$, the value of $d E_{G} / d T d e c r e a s e s$, and the sign changes as well (the threshold corresponds to a band edge energy of $1.1 \mathrm{eV}$ ). A similar trend is also identified for PbS NCs, for which $\mathrm{dE}_{\mathrm{G}} / \mathrm{dT}$ decreases and changes in size are observed. This effect is attributed to the size dependence of the electron-phonon coupling. 238
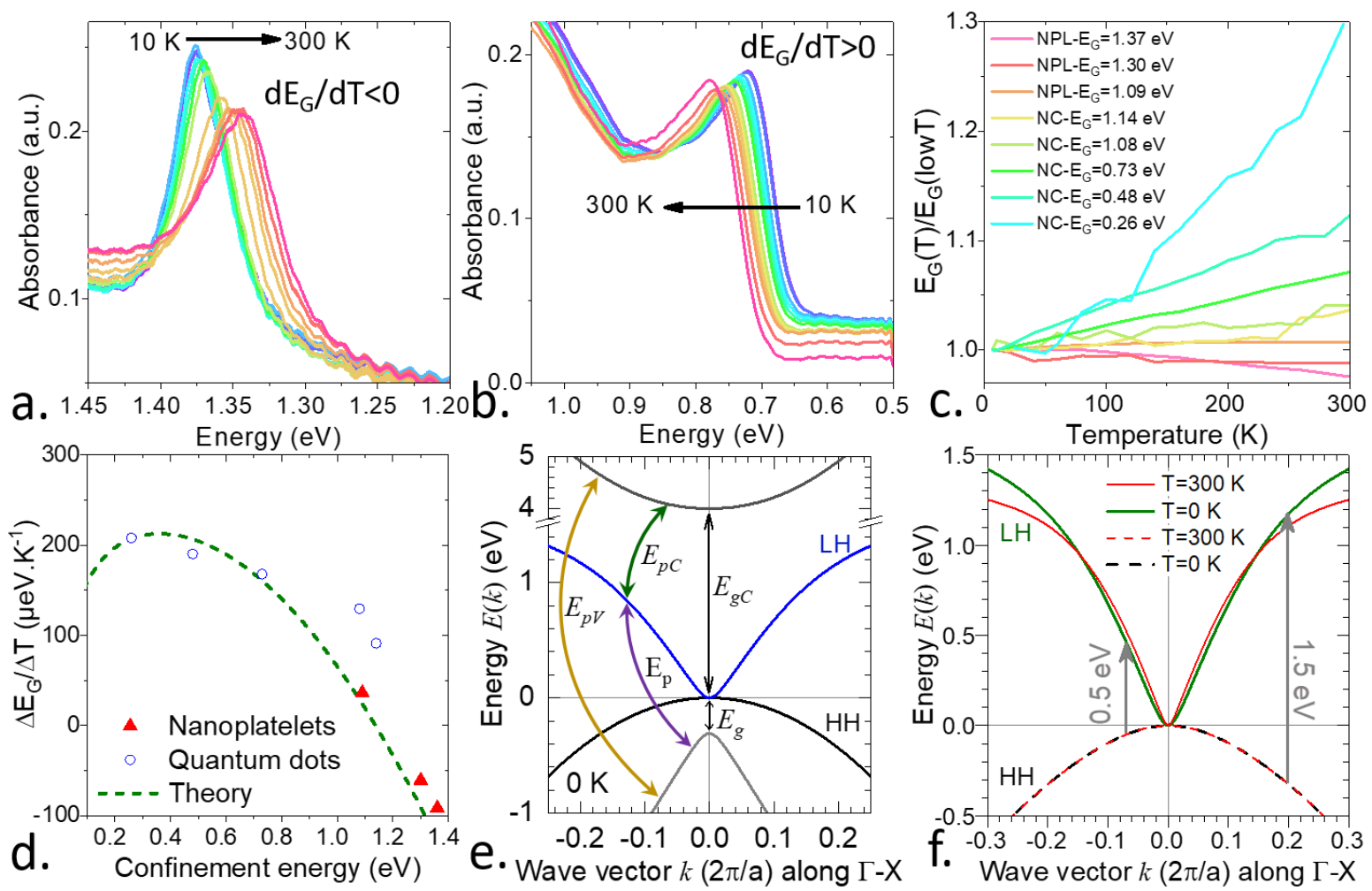

Figure 18 a. Infrared absorption spectra of HgTe NPLs at various temperatures between $10 \mathrm{~K}$ and 300 $K$ under zero pressure. The excitonic feature blueshifts as the temperature is lowered. $b$. Absorption spectra of $\mathrm{HgTe} N \mathrm{NCs}$ with a band edge at $6000 \mathrm{~cm}^{-1}$ for various temperatures between $10 \mathrm{~K}$ and 300 $K$ under zero pressure. $c$. Experimental shift of the bandgap as a function of temperature for different sizes of HgTe NCs and $3 \mathrm{ML} \mathrm{HgTe} N P L s$ with different surface chemistries. $d . d E_{G} / d T$ as a function of the confinement energy for the same set of samples. The simulated value of $d E_{G} / d T$ extracted from the pseudo k.p modeling is plotted as the green dashed line. e. Dispersion relation $E(k)$ of the conduction band of bulk HgTe extracted from the 3 band pseudo k.p model. $f$. Effect of temperature on $E(k)$ when the two bandgaps change with temperature. The gray arrows define the typical confinement energies. The dashed curves are the temperature and pressure independent valence bands assumed in the confinement model. The figures are adapted with permission from ref ${ }^{62}$ Copyright (2020) American Chemical Society.

To clarify the mechanism of the coupling of confinement and temperature dependence, Moghaddam et al. ${ }^{62}$ used a 14 band $k \cdot p$ model and a simplified 3+1-band model depicted in Figure 18e. The obtained band structure clearly demonstrates the nonparabolicity of the conduction band. The effective mass approximation only holds over $<1 \%$ of the Brillouin zone. However, for strongly confined NPLs ( $\approx 1.5 \mathrm{eV}$ of confinement energy), the dispersion relation must be correctly described for large wave vectors. In particular, this model includes the second conduction band, which is $>4 \mathrm{eV}$ above the heavy hole band. This band plays a key role. As the temperature is increased, the bandgap $E_{g c}$ shrinks, causing the second conduction band to approach the first 
one. To avoid an intersection, the first band bends downward. Because of this temperature dependent curvature, the effect of the temperature on the band edge is different for small and large wave vectors (i.e., for large and small NCs, respectively), as shown in Figure 18f. It can be concluded that the temperature dependence reflects the dispersion relation and that $\mathrm{dE}_{\mathrm{G}} / \mathrm{dT}$ is directly related to the measurement of the band curvature.
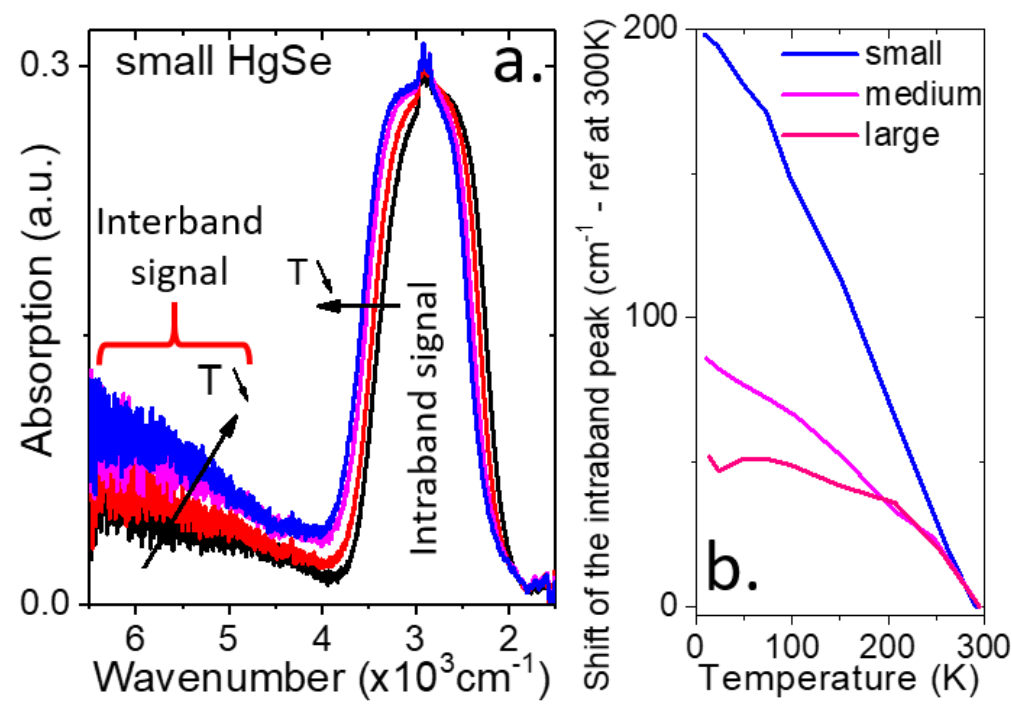

Figure 19 a. Absorption spectra of small CQDs at temperatures decreasing from 300 to $4 \mathrm{~K}$. b. Shift of the intraband/plasmonic peak as a function of temperature for three sizes of HgSe CQDs. The figure is adapted with permission from ref ${ }^{122}$ Copyright (2017), American Chemical Society.

To complete the discussion regarding the temperature dependence on the spectroscopic properties of HgX NCs, it is essential to mention that the intraband transition blueshifts upon cooling. ${ }^{34,122}$ This shift is also dependent on the NC size, as the shift is larger $\left(\approx 80 \mu \mathrm{eV} . \mathrm{K}^{-1}\right)$ for smaller NCs. This size dependence is related to the change in the nature of the transition as the size increases. In HgSe, small NCs are less doped and present a true intraband transition, ${ }^{29}$ while the largest NCs are more degenerately doped and the transition is more plasmonic. ${ }^{136}$

\subsubsection{Effect of pressure}
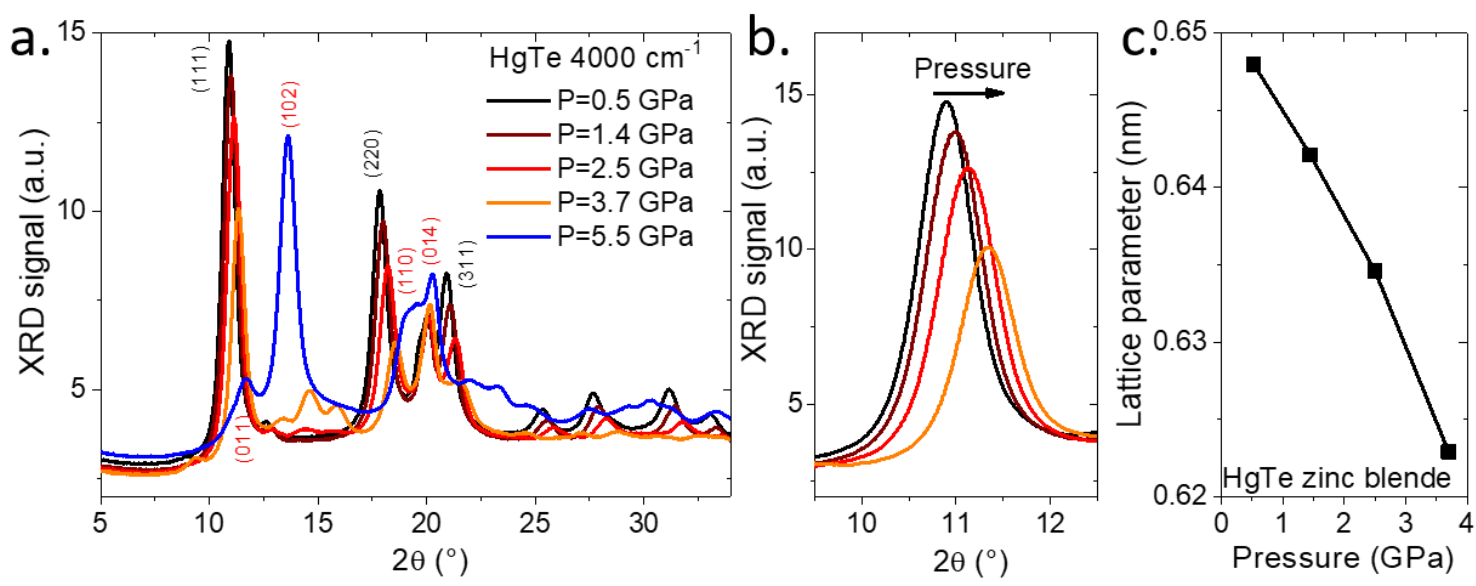

Figure 20 a. Diffraction pattern of HgTe CQDs (with the band edge at zero pressure being $4000 \mathrm{~cm}^{-1}$ ) under different pressures. b. Zoomed in view of the (111) Bragg reflection of HgTe CQDs (with the band edge at zero pressure being $4000 \mathrm{~cm}^{-1}$ ) under different pressures while the material remains in the zinc blende phase (i.e., $P<4$ GPa). c. Lattice parameter of the zinc blende phase as a function of pressure. The figure is adapted with permission from ref ${ }^{240}$ Copyright (2019), American Chemical Society. 
As stated above, the interest in pressure studies is driven by the growth of heterostructures. Over recent years, significant effort has been dedicated to the growth of $\mathrm{CdTe} / \mathrm{HgTe}$ quantum wells and superlattices to realize topological insulators. ${ }^{241,242}$ Pressure modulation has been identified as a strategy to finely tune the energy landscape of such heterostructures. ${ }^{243,244}$ Similar progress has been made for the growth of colloidal heterostructures based on HgX NCs; see the section dedicated to heterostructures. This aspect includes shelling with a CdX layer to design type $\mathrm{I}^{136,185,187}$ and type II band alignments to enhance the PL efficiency and to realize transport applications ${ }^{166}$ and enhanced charge dissociation in $\mathrm{HgSe} / \mathrm{HgTe}$ intraband detectors, respectively. ${ }^{120}$

The first question raised by the application of pressure is the existence of a domain of the zinc blende phase in which HgX NCs crystallize at room pressure. Bulk HgTe undergoes a transition from zinc blende to cinnabar ${ }^{245,246}$ phase at $1.4 \mathrm{GPa}$ and to the rock salt phase at approximately $8 \mathrm{GPa} .{ }^{247}$ In $\mathrm{HgSe}$, similar phase changes are observed, albeit with different pressure thresholds. ${ }^{248}$ The value of $1.4 \mathrm{GPa}$ typically falls in the range of pressures that are induced by the growth of a shell on a core if the two material lattices are not matched. ${ }^{249}$ Livache et al. ${ }^{240}$ estimated that the growth of a CdS shell on a HgTe core (10\% lattice mismatch) should lead to a pressure of $2.6 \mathrm{GPa}$ on the core, enough for the bulk to induce a structural phase transformation. Thus, by applying pressure on HgX NCs, it can be determined whether a change in the spectroscopic properties is the result of a phase change. Livache et al. investigated the pressure effect on a series of HgTe and HgSe NCs on both inter- and intraband transitions. ${ }^{240} \mathrm{X}$-ray diffraction under pressure indicated that the zinc blende phase exists at least up to $3.7 \mathrm{GPa}$, as shown in Figure 20a. This delay in the phase transition compared to the bulk is attributed to a nucleation barrier. ${ }^{250}$ Additionally, a similar behavior (with measurement resolution) has been observed for NCs and NPLs. ${ }^{62,240}$ At $4 \mathrm{GPa}$, the two phases coexist, although it is unclear whether this coexistence occurs within a single NC or if various NCs undergo phase changes at slightly different pressures. By fitting the shift of the diffraction peak in the zinc blende phase (Figure 20b and c), the change in the lattice parameter can be estimated to be $10 \mathrm{pm} / \mathrm{GPa}$, corresponding to a bulk modulus for HgTe NCs of $33 \mathrm{GPa}$. The same value holds for HgTe NPLs ${ }^{62}$ and corresponds to the bulk value, ${ }^{251}$ suggesting that the ligands do not considerably influence the mechanical properties of the NC film.

To apply pressure, the sample is loaded in a diamond anvil cell in a pressure transmission medium (alcohol or infrared transparent salt for diffraction and infrared spectroscopy, respectively), whose role is to ensure pressure transmission to the sample. In addition, a ruby is inserted within the cell, whose luminescence is used as a pressure gauge; see the scheme of the setup in Figure 21a. As pressure is applied, the infrared spectrum of HgTe NCs is dramatically affected (see Figure $21 \mathrm{~b}$ and d). A shift as large as $300 \mathrm{meV}$ can be observed under $4 \mathrm{GPa}$ of pressure. In this sense, the application of pressure is more dramatic than the temperature effect. For the sake of a comparison, it can be considered that cooling a HgTe sample by $300 \mathrm{~K}$ shifts the band edge by less than $60 \mathrm{meV}$ (see Figure 18b and d). Applying pressure induces a blueshift of the interband transition (see Figure 21b and c). This shift toward high energy occurs for any confinement value, although the magnitude of the shift is slightly dependent on the confinement energy: $100 \mathrm{meV} / \mathrm{GPa}$ for NPLs as opposed to $60 \mathrm{meV} / \mathrm{GPa}$ for $8 \mathrm{~nm}$ NCs. Using the same (3+1) band k.p model used for temperature, Moghaddam et al. ${ }^{62}$ demonstrated that the pressure effect is mostly driven by the bulk $\Gamma_{8}-\Gamma_{6}$ bandgap ( $E_{G}$ in Figure 18e), which starts from a negative value at zero pressure and finally opens up for pressures above $2.6 \mathrm{GPa}$ as the band ordering recovers the normal order (i.e., $\Gamma_{6}$ above the $\Gamma_{8}$ band). Additionally, note that above $4 \mathrm{GPa}$ (in the cinnabar phase), the excitonic transition fully disappears. However, this process is reversible with a strong hysteresis for pressures up to $10 \mathrm{GPa}$.

Moreover, the intraband transition is influenced by the pressure application; see Figure $21 \mathrm{~d}-\mathrm{e}$. The shift is the opposite to that observed for the interband transition (i.e., a redshift upon pressure application). The absolute magnitude of this shift is smaller (< $10 \mathrm{meV} / \mathrm{GPa}$ ). This shift can be attributed to the increased effective mass in the conduction band. ${ }^{240}$

Distinct behaviors such as the pressure effect, decrease in the magnitude of the transition, and disappearance of the transition induced by phase transformation must be considered for the future design of $\mathrm{HgX}$ based heterostructures. 

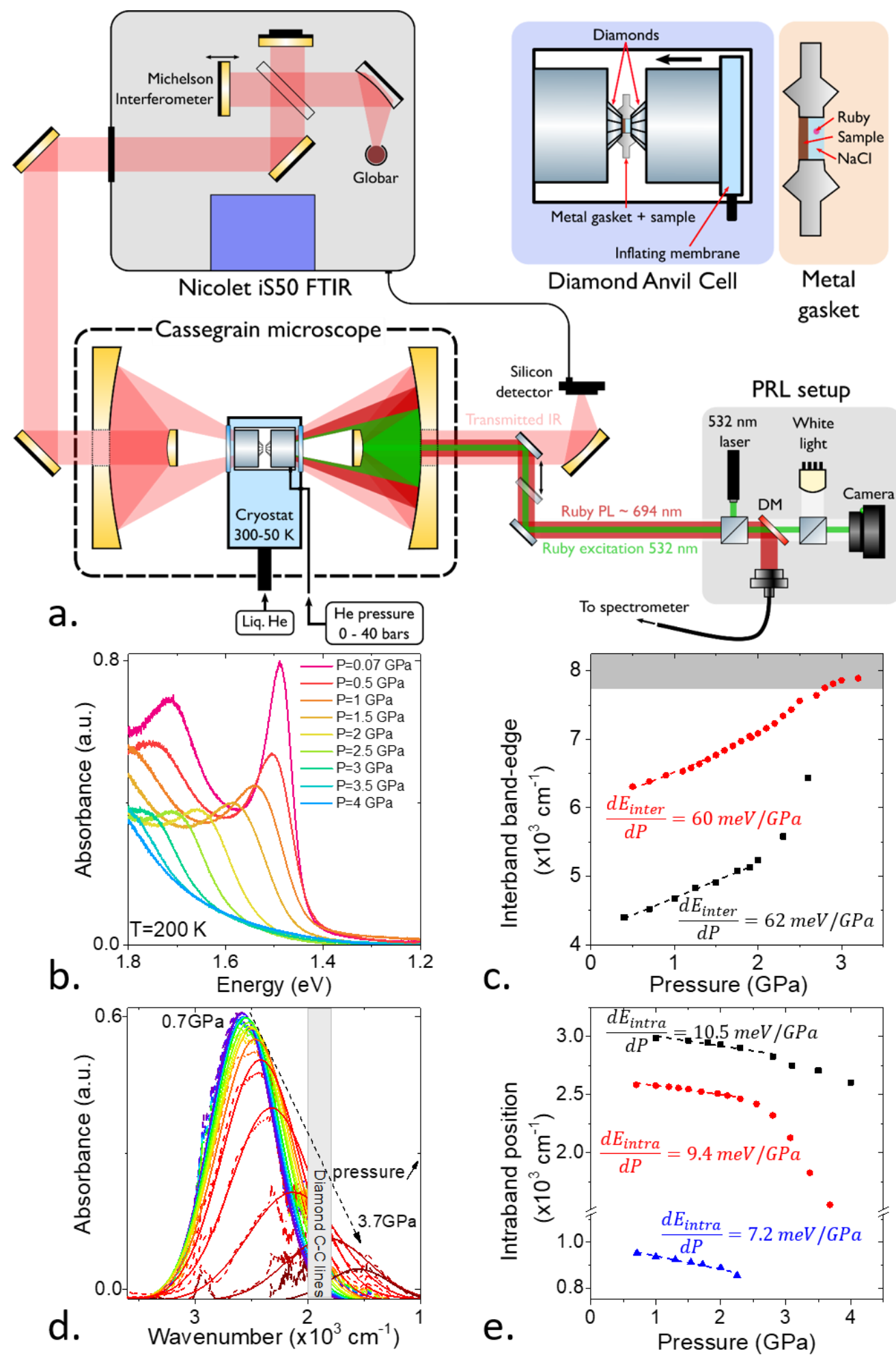

Figure 21a. Scheme of the experimental setup used to examine the infrared spectrum of HgTe NPLs under pressure. PRL: pressure according to ruby luminescence. DM: dichroic mirror. $b$. Infrared spectrum of HgTe NPLs at $200 \mathrm{~K}$ under various pressures ranging from 0 to $4 \mathrm{GPa}$. Parts a and $b$ are adapted from ref ${ }^{62}$ Copyright (2020), American Chemical Society. C. Interband band edge energy as a function of the applied pressure for two populations of HgTe CQDs with band edges of approximately $4000 \mathrm{~cm}^{-1}$ (in black) and $6000 \mathrm{~cm}^{-1}$ (in red). d. Infrared spectrum of HgSe CQDs with a zero pressure intraband feature at $2500 \mathrm{~cm}^{-1}$ under various pressures. e. Intraband band edge energy as a function of the applied pressure for three populations of $\mathrm{HgSe} C Q D s\left(3000 \mathrm{~cm}^{-1}\right.$ in black, $2500 \mathrm{~cm}^{-1}$ in red and $1000 \mathrm{~cm}^{-1}$ in blue). Parts $\mathrm{c}-\mathrm{e}$ are adapted with permission from ref ${ }^{240}$ Copyright (2019), American Chemical Society. 


\subsection{Experimental determination of the band alignment}

Infrared optical spectroscopy is the most convenient strategy to measure the band edge energy and possibly higher transition energies when sufficiently monodisperse syntheses are employed (see Figure $5 b$ ). However, such techniques are differential methods and do not provide insights into the density of the states of each band or absolute band alignment (i.e., relative position of the band vs. the vacuum or Fermi level). The latter information is critical to understand the charge delocalization in heterostructures and to design vertical geometry devices. For example, reducing the Schottky barrier at the metal-semiconductor interface is critical to avoid losses. Another example is the measurement of the dipole-induced shift resulting from ligand exchange, which is typically used for the design of $p-n$ junctions. Consequently, additional experimental methods must be developed. Tunnel spectroscopy $y^{252-255}$ is one such approach, which can be used to correlate spectroscopic data with structural information. The method has been applied to a variety of nanocrystals, including lead chalcogenides, ${ }^{256} \mathrm{II}_{\mathrm{V}} \mathrm{VI}^{257}$ and III-V semiconductors. ${ }^{258}$ However, to date, tunnel spectroscopy remains mostly unused in the case of HgX NCs. The only report related to on-chip tunnel spectroscopy is discussed in terms of a single particle field effect transistor in the chapter dedicated to transport, ${ }^{259}$ as shown in Figure 33.

Two methods appear to be of key interest to measure the band alignment of NC materials: photoemission and electrochemistry (see Figure 22). Photoemission is the conventional approach to measure the band alignment of bulk semiconductors. ${ }^{260}$ Electrochemistry is a more chemistry-oriented method ${ }^{261}$ that exploits the large porosity of NC films. Both methods have been applied to NCs and, in particular, to HgX NCs. ${ }^{68,192,122}$ However, both techniques exhibit certain limitations. The ultrahigh vacuum used for photoemission is not representative of the operating conditions of the device, even though this aspect is more notable for solar cells than for a HgTe NC-based IR sensor that is likely operated under secondary vacuum in a cryostat. Similarly, in the case of electrochemistry, the use of an electrolyte is not representative of the final NC environment in the device. A systematic comparison of the two methods might be useful but has not been reported yet.

\subsubsection{Photoemission}

To conduct photoemission measurements, a UV or X-ray photon is projected on the sample, with a typical energy of more than the material work function (see Figure 22b). Consequently, a photoelectron is extracted from the sample, and an electromagnetic lens later injects this electron into an analyzer used to determine its kinetic energy $\left(E_{K}\right)$. Owing to the short escape depth of the electrons in the material (a few $\mathrm{nm}$ ), this experiment must be conducted under ultrahigh vacuum. A scheme of the setup is given in Figure 22a. According to the energy conservation law, $h v=E_{B E}+E_{K}+W F_{\text {analyzer }}$, where $h v$ is the photon energy and $W F_{\text {analyzer }}$ is the work function of the analyzer. This equation is used to convert the experimental scale $E_{K}$ into a material scale at the binding energy $E_{B E}$.

Large photon energies (1486 eV from the Al $\mathrm{K}_{\alpha}$ line for the laboratory setup) are employed to analyze the core levels. X-ray photoemission spectroscopy (XPS) was used to analyze the chemical composition of the samples and determine the oxidation states of each atom. In the case of $\mathrm{HgX} \mathrm{NCs,} \mathrm{the} \mathrm{XPS} \mathrm{analysis} \mathrm{consistently} \mathrm{clarified} \mathrm{two} \mathrm{contributions} \mathrm{for} \mathrm{the} \mathrm{Hg} 4 \mathrm{f}$ state, resulting from the core and surface $\mathrm{Hg}$ excess. ${ }^{122,262}$ Thiol is generally used for the surface chemistry technique, and $\mathrm{S}$ is more electronegative than other chalcogenides. The surface contribution appears smaller and occurs at a higher energy. The XPS signal associated with the chalcogenides generally appears as a single contribution. ${ }^{122,262}$ By tuning the photon energy using synchrotron radiation, the contributions of the bulk and surface can be tuned.

To analyze the valence band, a lower photon energy is generally used, as obtained from a He lamp ( $h v=21.2 \mathrm{eV}$ ) in a laboratory or from low photon energy in a synchrotron ( $h v<100 \mathrm{eV}$ typically). This condition is typically employed to determine the work function and position of the valence band with respect to the Fermi level. In photoemission, the Fermi level is assigned as the zero binding energy. In practice, a clean metallic substrate is used to calibrate the energy scale. The low binding energy part of the photoemission spectra is fitted with a Fermi Dirac distribution. If necessary, a correction is introduced to set the Fermi energy to zero, and this correction is later applied to the whole XPS spectra. Alternative calibration methods may also involve a core level of a well-defined binding energy ( $\mathrm{C} 1 \mathrm{~s}$ or $\mathrm{Au} 4 \mathrm{f}$ are typically used). In a semiconductor such as $\mathrm{HgTe}$, the signal associated with the valence band appears to shift from the Fermi level (see Figure 22d). One can determine $V_{B}-E_{F}$ (i.e., the energy of the valence band with respect to the Fermi level) from a linear extrapolation of the photoemission signal to the baseline. It is critical to conduct this extrapolation while the photoemission signal is on a log scale; otherwise, the value of $V_{B}-E_{F}$ will be systematically overestimated, which makes the semiconductor appear more $n$-type than it is. The other valuable information that is extracted from this spectrum is the work function. The work function is associated with the electrons with the lowest kinetic energies, owing to which an additional external bias must be applied to allow these low kinetic energy electrons to move up to the analyzer. The kinetic energy associated with the cutoff of the secondary electrons is measured (see Figure 22c), and this value is related to the material work function. For HgTe and HgSe NCs, all the reported data ${ }^{49,122,192,262}$ match $W F=4.6 \pm 0.1 \mathrm{eV}$ for EDT-capped NCs. 


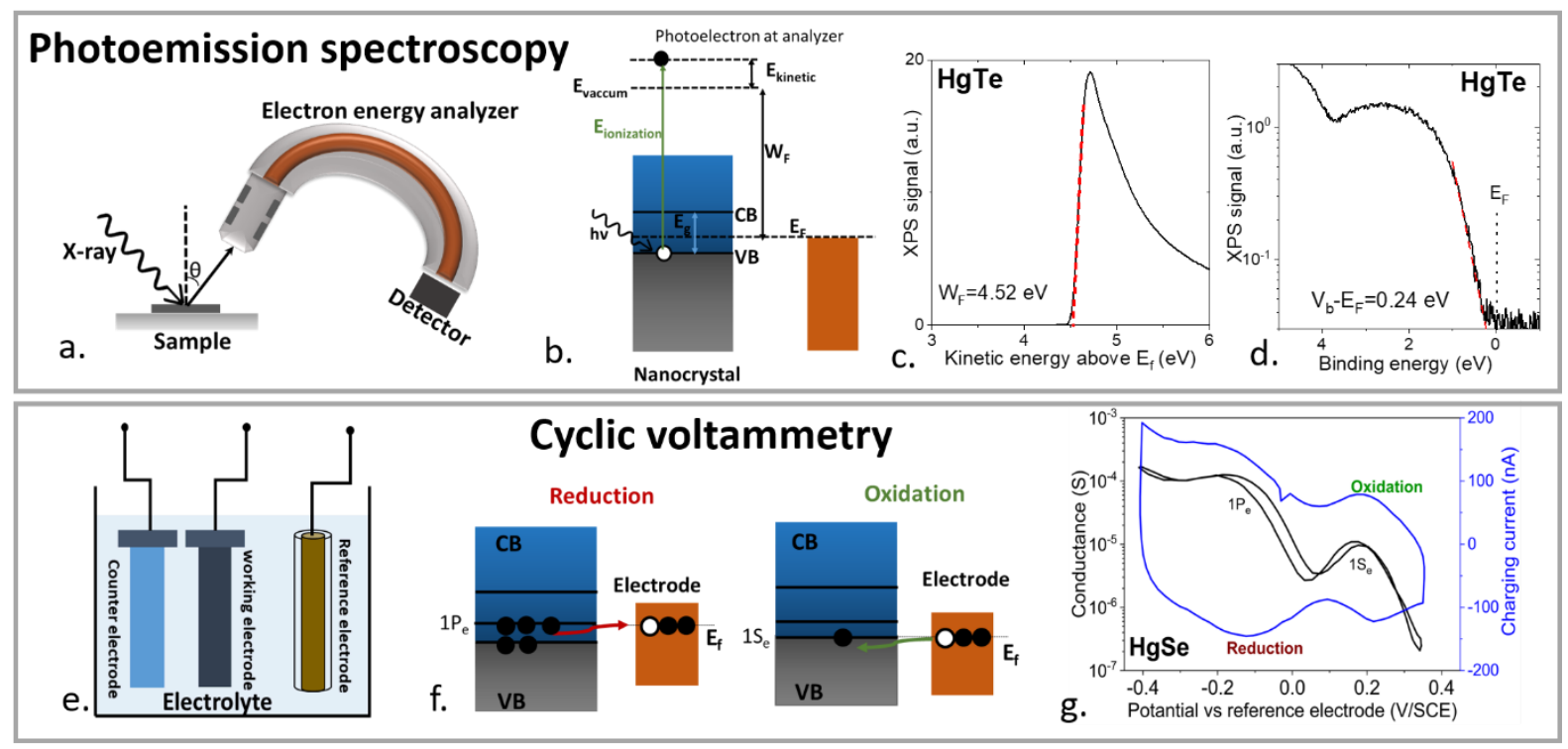

Figure 22 a. Schematic of the X-ray photoemission experimental technique. $b$. Representation of the energy level diagram demonstrating the principle of $X$-ray photoemission. $X$-ray photoemission spectrum relative to the cutoff of secondary electrons (c) and those from the valence band (d) for a $\mathrm{HgTe}$ nanocrystal film. The band energies are determined from the intersection of an extrapolation from the signal to the baseline. e. Schematic of the cyclic voltammetry experimental setup. $f$. Scheme of the energy level diagram demonstrating the reduction and oxidation mechanism employing cyclic voltammetry $\mathrm{g}$. Cyclic voltammetry of a HgSe nanocrystal film. Part $\mathrm{g}$ is adapted with permission from ref ${ }^{68}$ Copyright (2017), American Chemical Society.

A critical aspect to obtain reliable measurements is to avoid the charging of the material. As photoelectrons escape from the sample, they leave positive charges behind, inducing an electrostatic shift. To allow rapid charge compensation and avoid this charging effect, NC films are generally deposited on a conductive metallic substrate (typically, gold). Moreover, the thickness of the film is a critical aspect: if the film is not sufficiently thick, then it will be discontinuous and a signal from the substrate will be observed. This aspect is generally not detrimental for core levels but can be problematic when determining the valence band. However, if the film is excessively thick and not sufficiently conductive, then the top part will not be connected to the conductive substrate and will be susceptible to charging. This aspect may lead to the observation of two contributions for each core level: a shifted one from the charged parts of the sample and a nonshifted one for the parts in which the sample is more electrically connected. The sample resistance thus appears to be a major concern. It is critical to process the nanocrystals as if they were used for transport (i.e., to conduct ligand exchange). In this approach, not only is the measurement representative of the actual environment within the device but the charging effect is also strongly reduced. Nevertheless, these concerns must be mitigated for HgX NCs, for which the film resistance is considerably smaller than that for wider bandgap materials and for which charging is not systematic even with weakly electrically coupled NCs.

Once the $V_{B}-E_{F}$ and $W F$ values have been determined and the interband band edge energy $E_{G}$ is known, the energy landscape of an NC population (see Figure $23 \mathrm{~b}, \mathrm{c}$ and d) can be reconstructed. The valence band and vacuum level are localized with respect to the Fermi level, while the conduction band is localized with respect to the valence band by using the measured optical bandgap. In this case, it is assumed that the Coulombic correction of the optical spectrum is small and negligible, as verified by tight binding simulations. ${ }^{216}$

\subsubsection{Electrochemistry}

Electrochemistry has long been used as a tool to determine the quantum states of NCs ${ }^{263-265}$ and was first applied to HgTe NCs by Liu et al., ${ }^{266}$ who demonstrated the ambipolar nature of MWIR (5 $\mu \mathrm{m}$ cutoff) HgTe NCs. To conduct electrochemistry, the film of NCs is deposited on a conductive substrate and dipped into an electrolyte, while two additional electrodes control the current and applied potential (see Figure 22e). As the current flows, the NCs become reduced or oxidized, thereby tuning the filling of the surface and quantum states of the NCs (see Figure 22f). A typical cyclic voltammetry diagram is plotted in Figure $22 \mathrm{~g}$. The bumps in the cyclic voltammetry curve are associated with the filling/unfilling of the quantum states. In this measurement, the energy reference is the reference electrode, whose potential must be constant and is later rescaled vs. vacuum. In this experiment, the 
Fermi level is the rest potential of the film in the open circuit condition. If two electrodes are present on the substrate of the sample, then it is possible to measure the conductance of the sample and carrier mobility, thereby providing additional information compared to what can be obtained from photoemission. A typical refinement of the cyclic voltammetry experiment is to couple the experiment with spectroscopy to conduct spectroelectrochemistry. In this case, it is possible to observe the bleach of the interband as the intraband peak appears ${ }^{193,266}$ and obtain a more accurate estimation of the quantum state filling as opposed to any other states that might be electrochemically filled and contribute to the electrochemical current.

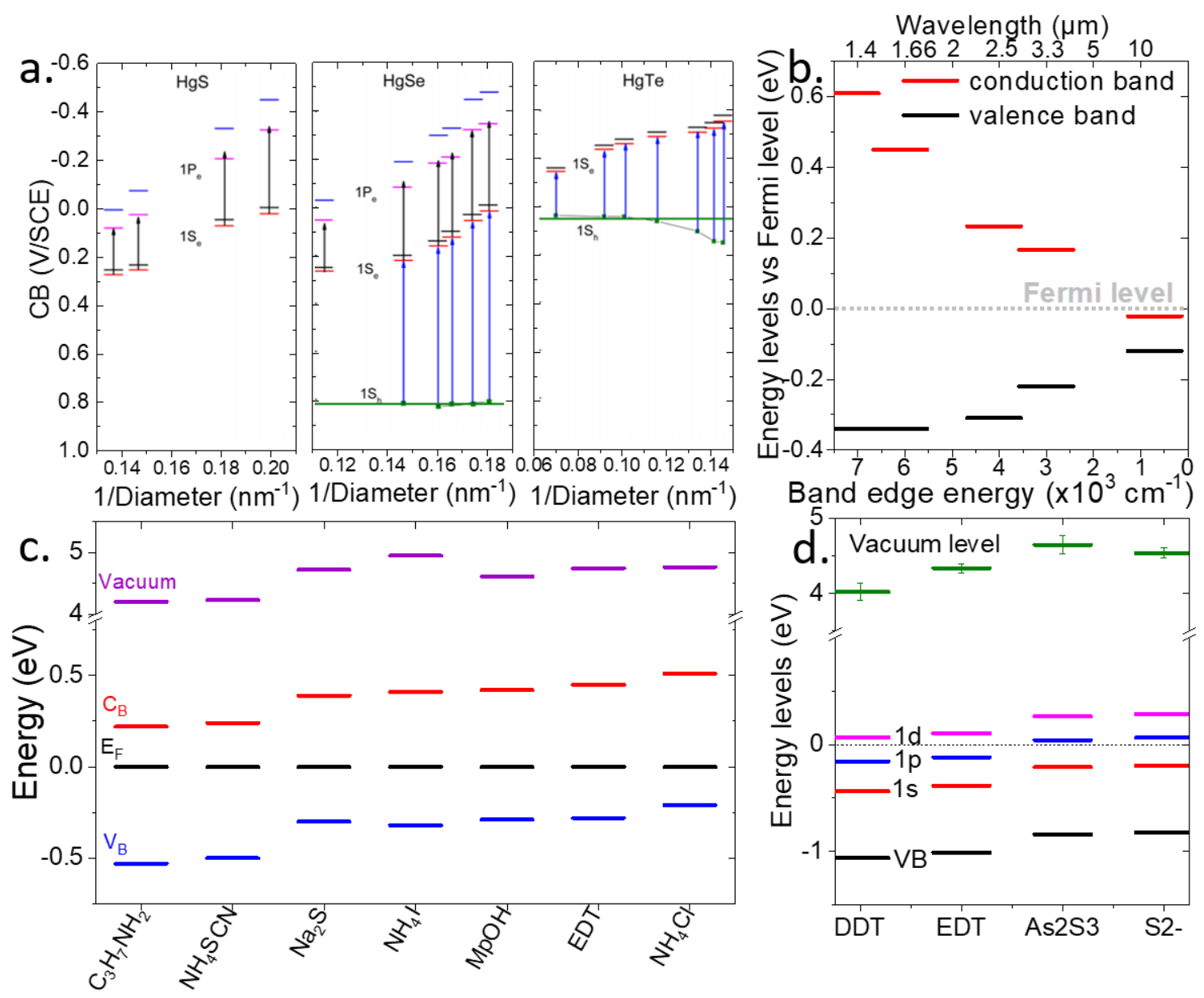

Figure 23 a. Electronic spectra of the HgX quantum states measured by electrochemistry before (black and blue represent $1 S_{e}$ and $1 P_{e}$, respectively) and after correction by the charging energy (red and pink represent $1 S_{e}$ and $1 P_{e}$, respectively. Intraband $1 S_{e}-1 P_{e}$ (the length of the black arrow) and interband $1 S_{h}-1 S_{e}$ (the length of the blue arrow) transitions measured through spectroscopy and corrected for the exciton binding energies are also indicated. Part a is adapted with permission from ref ${ }^{68}$ Copyright (2017), American Chemical Society. b. Band alignment of HgTe nanocrystals with different band edge energies. Part $b$ is adapted with permission from ref ${ }^{192}$ Copyright (2018), American Chemical Society. c. Energy of the valence band, conduction band and vacuum level for $\mathrm{HgTe}$ nanocrystals with a $1.7 \mu \mathrm{m}$ cutoff wavelength and different capping ligands. The Fermi level is set as $0 \mathrm{eV}$. Part $\mathrm{c}$ is adapted with permission from ref ${ }^{49}$ Copyright (2019), American Chemical Society. $d$. Electronic spectrum (black, red, blue, pink, and green correspond to the valence band, $1 S_{e}$ state, $1 P_{e}$ state, $1 D_{e}$ state, and vacuum level, respectively) for medium HgSe CQDs capped with four different ligands. Zero on the energy scale corresponds to the Fermi level. Part $c$ is adapted with permission from ref ${ }^{122}$ Copyright (2017), American Chemical Society.

In regard to $\mathrm{HgX}$ NCs, systematic studies have been conducted as a function of the material composition, ${ }^{68}$ size ${ }^{68,192,122}$, and surface chemistry, $^{122,49}$ as shown in Figure 23 . In the event of marginal quantitative disagreements between the electrochemistry and photoemission techniques, the following quantitative trends can be considered for future discussion:

- Band alignment: As chalcogenides become heavier (atomic mass), the maximum valence moves toward the vacuum level. Using the electrochemistry approach, ${ }^{68}$ the energies of the top of the valence band are determined to be 5.85 ( $\mathrm{HgS}$ ), 5.5 (HgSe) and $4.77 \mathrm{eV}(\mathrm{HgTe})$ below the vacuum level. The values obtained from photoemission are $5.3 \mathrm{eV}$ and $4.85 \mathrm{eV}$ for 
$\mathrm{HgSe}^{122}$ and $\mathrm{HgTe},{ }^{192}$ respectively, corresponding to a slightly reduced band offset. The trend of the conduction band is slightly more complicated due to the presence of a bandgap in $\mathrm{HgS}$, leading to the bottom of the conduction band energy being 5.2 (HgS), 5.5 (HgSe) and $4.77 \mathrm{eV}(\mathrm{HgTe})$ below the vacuum level. ${ }^{68}$ As mentioned previously, there is reasonable agreement regarding the work function values, with values of $4.7 \mathrm{eV}$ and 4.4-4.6 eV obtained using electrochemistry ${ }^{68}$ and photoemission for both $\mathrm{HgSe}$ and $\mathrm{HgTe}$ for the same capping ligands (EDT), respectively.

- Size effect: Size, through confinement, influences the relative position of the Fermi level and bands. In $\mathrm{HgS}$ and $\mathrm{HgSe}, \mathrm{for}$ sizes above $3 \mathrm{~nm}$, the Fermi level is in the conduction band. This phenomenon corresponds to degenerate doping, which is consistent with the observation of an intraband feature in the infrared spectra of these materials (see Figure 23d). Nevertheless, the Fermi level can be found to be lower in this band depending on the size, barely filling the $1 S_{e}$ level for the smallest particles, and it is above the $1 \mathrm{D}_{\mathrm{e}}$ state for the largest particles, ${ }^{122}$ as shown in Figure $29 \mathrm{~b}$. This shift is responsible for a metal-semiconductor transition, as discussed in the transport part of this review. In the case of HgTe, the Fermi level is almost resonant with the conduction band, and the size effect can be even more dramatic (see Figure 23b). For small confined particles, the Fermi level is within the bandgap, closer to the valence band (i.e., p-type). As the particle size increases, the Fermi level reaches the upper part of the bandgap (n-type) and finally reaches the conduction band as the cutoff wavelength exceeds 5-10 $\mu \mathrm{m}(12-20 \mathrm{~nm}$ ) (see Figure 23b).

- Surface chemistry effect: As observed for PbS NCs, the band alignment is affected by the change in the capping ligands; see the section dedicated to doping. The library of ligands that has been tested remains smaller than that reported for $\mathrm{PbS}^{195,196,267}$ and is mostly focused on short thiols or short surface ligands compatible with transport (see Figure 29c and d). The shift of the bands induced by the change in the surface chemistry can reach $0.3 \mathrm{eV}$, which is, given the narrow bandgap nature of the material, of the order of $E_{g} / 2$. A value of $0.3 \mathrm{eV}$ is sufficient to switch $\mathrm{HgTe}$ from the $n$ - to $p$-type (see Figure 29c) or to switch from degenerate doping to a Fermi level within the bandgap in the case of HgSe (see Figure $13 a$ and $b)$.

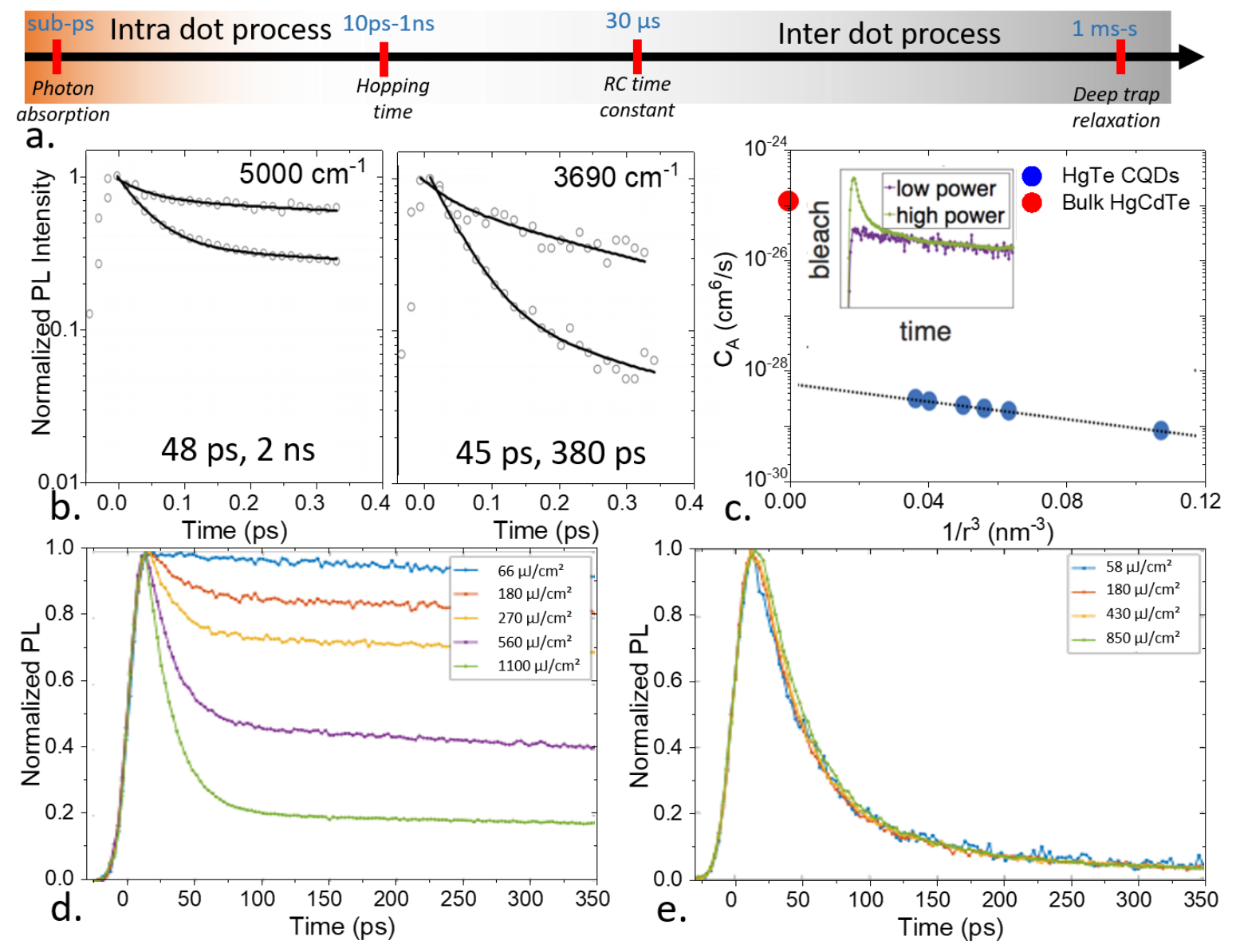

Figure 24 a. Time scale of the different carrier relaxation times in a film of HgTe NPLs b. PL decays for HgTe CQDs emitting at $2 \mu \mathrm{m}$ (left) and $2.7 \mu \mathrm{m}$ (right). The open circles denote the measured intensity; the solid lines represent the biexponential fits with the time constants presented as labels. The samples were excited with $1.2 \mathrm{~mW}$ (high power) and $0.027 \mathrm{~mW}$ (low power). Part b is adapted with permission from ref ${ }^{168}$ Copyright (2014), American Chemical Society. c. Magnitude of the Auger coefficient as a function of the inverse of the particle volume for HgTe NCs. Part $c$ is adapted with 
permission from ref ${ }^{268}$ Copyright (2018), American Chemical Society. d. Interband fluorescence decay at $5600 \mathrm{~cm}^{-1}$ for self-doped HgSe NCs. e. Intraband fluorescence decay at $2100 \mathrm{~cm}^{-1}$ for self-doped $\mathrm{HgSe}$ NCs. Parts $d$ and e are adapted with permission from ref ${ }^{269}$ Copyright (2019), American Chemical Society. 


\subsection{Carrier dynamics}

The highly specific size-dependent electronic structures of mercury chalcogenide NCs as well as the semimetal nature of the bulk material are expected to impact carrier relaxation, especially compared to that of wider bandgap nanocrystals. The stronger density of states is expected to allow efficient phonon-assisted relaxation, and the higher doping level may accelerate Auger relaxation. Carrier relaxation is of interest for both light emission and detection efficiency, and consequently, this aspect is key to examine the carrier relaxations in HgX NCs. Time-resolved methods require a setup in which both the source and detection are designed to operate in the infrared region. Time-resolved optical methods, such as time-resolved photoluminescence (TRPL), appear to be well suited for examining processes that occur at short time scales (a few ps to 100 ns), corresponding mostly to intra-NC relaxation (see Figure 24a). Note that time-resolved transient absorption (TA) is mostly unexplored for HgX NCs. ${ }^{270}$ This aspect is paradoxical from a material viewpoint since the absorption signal does not degrade with increasing wavelength, as opposed to PL. To achieve larger time scales ( $\mu$ s to $s$ ), typically to understand the relaxation at the NC array level, alternative methods must be used. In the case of HgX NCs, a transient photocurrent and pump probe photoemission have led to interesting results.

\subsubsection{Time-resolved photoluminescence}

TRPL is the most common strategy to measure carrier relaxation in NCs. The success of this approach strongly relies on the ability to achieve single particle measurements. Such time-resolved measurements with a limited number of photons are enabled by fast single photon detectors, such as avalanche photodetectors. However, pushing this experiment in the infrared domain and, in particular, above $1.7 \mu \mathrm{m}$ is highlight challenging, and only a few researchers have attempted this expansion. ${ }^{21,168}$ Consequently, there is a lack of consistent data, with PL lifetimes spanning from $50 \mathrm{ps}^{168}$ up to several tens of $\mu \mathrm{s}^{22}$ The limited amount of available data may lead to unfair comparison of the carrier relaxation times. Moreover, the experimental conditions differ from one study to another with different particle sizes (by at least a factor of two based on the diameter) or different excitation pulse durations ( $p s^{168}$ vs. $\mathrm{ns}^{22}$ pulses). At short time scales, a fast component (50 ps) exists, as shown in Figure $24 \mathrm{~b}$, whose magnitude strongly depends on the excitation power. This decay time also correlates to the fast component observed in TA (Figure 24d and e) and is attributable to the biexciton lifetime. ${ }^{218}$ At long time scales, an extremely long decay constant has been observed and attributed to trap states, according to Geiregat et al. ${ }^{22}$ The authors used extremely small HgTe NCs, which increased the relative contribution of the surface. Moreover, the authors proposed using these trap states to design a three-level system leading to a low excitation power stimulated emission. ${ }^{22}$ In the intermediate range (100 ps to $100 \mathrm{~ns}$ ), the contribution from the exciton was identified. However, this contribution decreased promptly with NC size, from $500 \mathrm{~ns}$ for a band edge at $1.2 \mu \mathrm{m},{ }^{22}$ to $10-20 \mathrm{~ns}$ for a band edge at $1.7 \mu \mathrm{m},{ }^{21}$ to $2 \mathrm{~ns}$ at $2 \mu \mathrm{m}$ and to $400 \mathrm{ps}$ for emission at $2.7 \mu \mathrm{m} .{ }^{168}$ This rapid drop in the exciton lifetime strongly correlates with the decrease in the PL efficiency with increasing emission wavelengths. This reduction in the exciton lifetime is the result of a higher coupling to the ligands, especially as the PL signal starts to overlap with the absorption of the $\mathrm{C}-\mathrm{H}$ bonds, enabling efficient near-field energy transfer. ${ }^{21,168,271}$

\subsubsection{Transient $P L$ to examine the Auger relaxation}

The Auger relaxation of the interband transition in $\mathrm{HgTe}^{268}$ and $\mathrm{HgSe}^{269}$ presents a decay time of approximately 20-30 ps (see Figure $24 d$ ). This lifetime follows the volume scaling observed in nanocrystals. ${ }^{272,273}$ However, the relative magnitude of the Auger relaxation quantized using the expression $C_{A}=\frac{V^{2}}{8 \tau_{B}}$, where $\mathrm{V}$ is the particle volume and $\tau_{B}$ is the biexciton lifetime, is strongly reduced compared to that in the bulk. ${ }^{268}$ This aspects can help reducing the number of nonradiative decay paths, which may lead to brighter HgTe NCs and a reduction in the noise magnitude in IR detectors. ${ }^{58} \mathrm{~A}$ key feature is related to the relaxation of the intraband in HgSe. While the interband decay appears to be similar for HgTe (undoped: $<<1$ carrier per NC) and HgSe (doped: $\approx 2$ carriers per NC), despite their different carrier densities, the intraband feature displays no or extremely slow Auger relaxation. In addition, the decay constant is independent of the change in the fluence; see Figure $24 \mathrm{e} .{ }^{269}$ This result is counterintuitive, as the Auger value is favored by high carrier densities; however, in this case, the dynamics are retarded for a transition that is induced by the high carrier density of the material. This slowing of the Auger decay is interpreted as the consequence of the density of states in the material. Future challenges in this direction are related to the investigation of the extremely early time scale ( $<50 \mathrm{ps})$, which remains unaddressed for HgX NCs to date.

\subsubsection{Pump probe photoemission}

Time-resolved photoemission is a pump-probed technique in which optical photons from a pulsed source are used to excite NCs, and X-ray photons, typically from a synchrotron source, are used as probes. ${ }^{274} \mathrm{~A}$ typical scheme of the experimental setup is shown 
in Figure 25a. In practice, the probe is used to acquire a core level photoemission spectrum at different times of the pump. The obtained core level spectra are then fitted with Gaussians, and the peak binding energy is plotted as a function of time. Due to this fitting procedure, a super-resolution of $\approx 5 \mathrm{meV}$ is obtained, which is an enhanced resolution compared to that ( $30-50 \mathrm{meV}$ ) obtained by static X-ray photoemission measurements. This method can be seen as a time-resolved transient photovoltage experiment that examines the surface band bending at the interface. ${ }^{275}$ This approach represents a powerful in situ method to probe the band alignment in optoelectronic devices. ${ }^{276,277}$ Under dark conditions, a nonstoichiometric semiconductor presents surface band bending. Under illumination, electron-hole pairs are generated, and consequently, minority carriers flow toward the surface due to band bending. The band bending is continuously reduced, and under a sufficiently large irradiance, flat band conditions can be obtained. Once the semiconductor returns to dark conditions, the majority carriers from the bulk flow toward the surface and recombine with the minority carriers accumulated on the surface, thereby restoring the initial band bending. This method provides two key pieces of information. (i) First, the nature of the majority carriers can be confirmed. The sign of the photoinduced shift is related to the nature of the majority carriers. An increase in the binding energy under illumination, as observed in Figure 25b, corresponds to the electrons acting as majority carriers. ${ }^{262}$ In this sense, the method is correlated with field effect transistor (FET) measurements without requiring any electrodes. The second (ii) key piece of information involves the carrier dynamics. The turn on and turn off time is related to the diffusion time of the minority and majority carriers, respectively. ${ }^{165}$

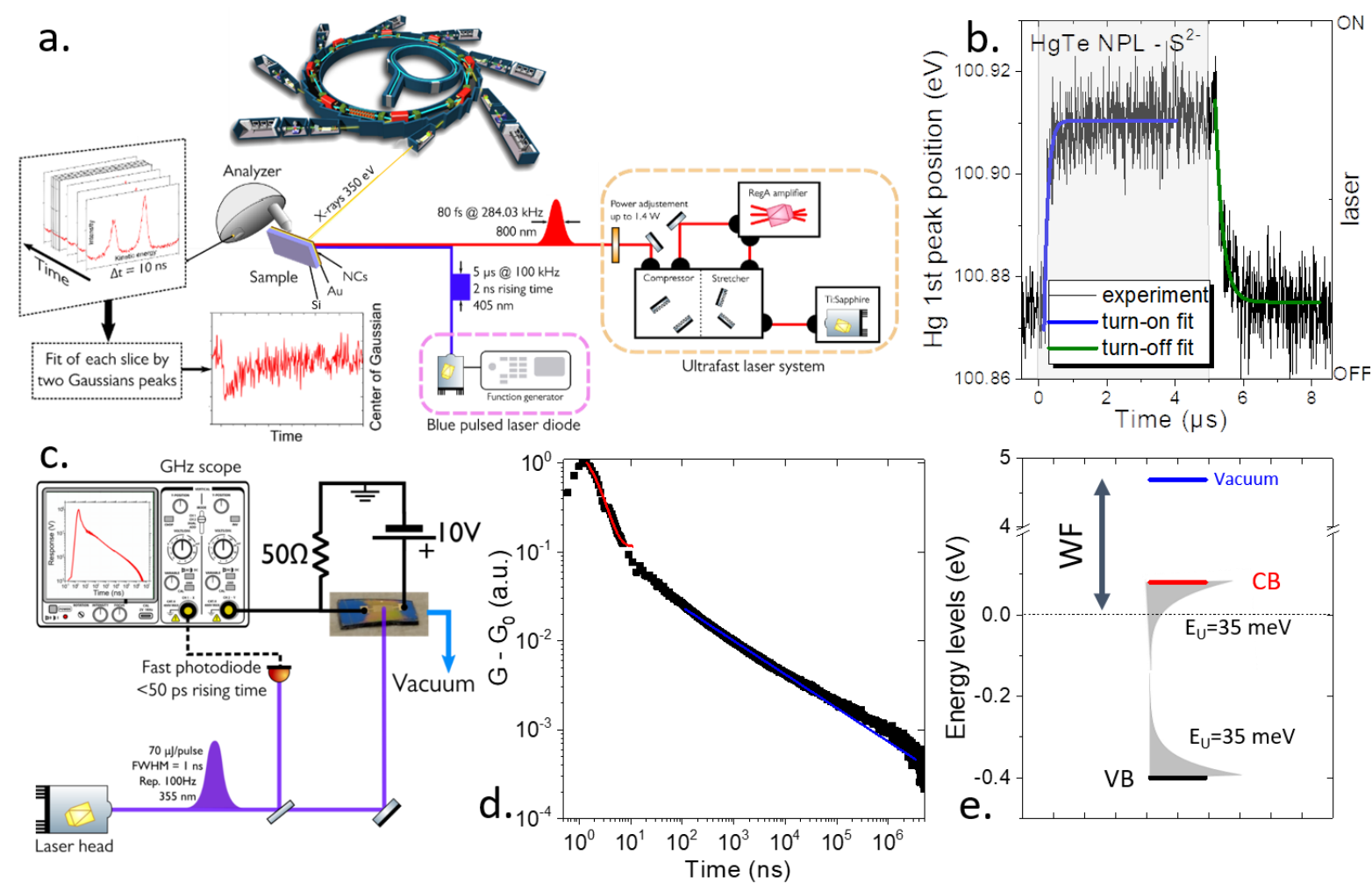

Figure 25 a. Scheme of the pump probe photoemission setup. Two laser sources can be used: an 80 fs amplified Ti sapphire laser operated at $800 \mathrm{~nm}$ or a $2 \mathrm{~ns}$ rise time blue $(405 \mathrm{~nm})$ laser diode. Light is projected on the sample under ultrahigh vacuum. X-rays at $350 \mathrm{eV}$ sourced from the Soleil synchrotron also illuminate the sample on the same spot. Every $10 \mathrm{~ns}$, a photoemission spectrum is acquired. The time resolution of the experiment is $30 \mathrm{~ns}$, limited by the analyzer. These spectra are fitted by two Gaussians, and the center of the low binding energy Gaussian is displayed as a function of time. $b$. The energy of the $\mathrm{Hg} 4 f_{7 / 2}$ core level fitted position as a function of time while the light (blue - $405 \mathrm{~nm}$ laser) is turned on and off for a HgTe NPL film capped with sulfide. Parts a and $b$ are adapted with permission from ref ${ }^{262}$ Copyright (2017), American Chemical Society. c. Transient photocurrent setup. (d) Evolution of conductance as a function of time after a $1 \mathrm{~ns}$ long pulse of light at $355 \mathrm{~nm}$. (e) Reconstructed energy spectrum including the trap distribution. Parts $c-e$ are adapted with permission from ref ${ }^{278}$ Copyright (2017), American Chemical Society.

Pump probe photoemission has been mostly applied to HgTe NPLs. Livache et al. used this approach as a strategy to clarify whether the response time of their device was limited by the material or device geometry. ${ }^{262}$ Gréboval et al. demonstrated that HgTe NCs and NPLs exhibited similar diffusion times for the majority carriers and concluded that the transport was mostly driven by confinement instead of the particle shape. ${ }^{165}$ 


\subsubsection{Transient photocurrent to examine the trap distribution}

As NCs exhibit low luminescence in the infrared region and on targeting time scales longer than a few ns, alternatives to TRPL and TA must be developed. The use of the transient photocurrent eliminates the constraints regarding the material luminescence and exploits the photoconductivity of $\mathrm{HgX} \mathrm{NC}$ arrays. Investigation of the transient photocurrent was driven by the determination of the response time for photodetectors. ${ }^{279}$ Most HgTe NC arrays exhibit a reasonably small response time. In general, responses of microseconds or less are commonly obtained for both photoconductive and photovoltaic devices based on HgTe (see Table 6 and Table 7, respectively). Intraband materials, however, present a slow response (ms-s range), which likely reflects the bolometric origin of the response for arrays of pristine HgSe NCs.

However, most short-pulsed sources are not available in the infrared region, and excitation occurs far above the band edge energy, ${ }^{279}$ which may result in a hot electron effect. ${ }^{280,281}$ Livache et al. demonstrated that multiexciton generation (MEG) occurs in HgTe illuminated by high photon energies. ${ }^{219}$ Typically, the threshold for MEG is between 3 and 5 times the band edge energy. To correctly examine the dynamics at the band edge, the authors used a pulsed quantum cascade laser, whose emission was resonant with the HgTe NC absorption. ${ }^{219}$ The authors demonstrated that a response time of as small as $20 \mathrm{~ns}$ (limited by the QCL) can be obtained from a conductive array of HgTe NCs.

Martinez et al. designed a specific setup to examine the transient photocurrent, as depicted in Figure 25c. ${ }^{278}$ Excitation was realized using a high power source enabling measurement of the current over several orders of magnitude. The excitation pulse was short to access fast dynamics; however, the repetition rate was maintained at a small value to examine the long time scale relaxation. This setup combined both broad temporal and signal dynamics. At short time scales, the authors observed a fast exponential decay of conductance followed by a long tail, ${ }^{282}$ see Figure $25 \mathrm{~d}$. In most setups dedicated to the detector response time, owing to the low signal resolution ( 1 or two orders of magnitude), only the early decay could be accessed before the photocurrent reached its baseline. Clarifying the slow component of the photocurrent required expansion of the signal dynamics by combining a strong excitation with sensitive detection. The hopping mechanism in HgTe NC arrays is estimated to be approximately a few nanoseconds. As a result, no internanoparticle transport can occur, and the current observed is the displacement current. ${ }^{280,281}$ The drop in current reflects electron-hole pair recombination and trapping. At long time scales, the observed power law decay is the signature of multitrapping transport, as previously observed in the case of amorphous materials. ${ }^{283,284}$ As transport starts, the carriers are first trapped and then thermally released. The release time reflects the depth of the trap. Consequently, the power exponent $(b)$ of the decay directly reflects the trap distribution though the relation $b=-1+\frac{k_{b} T}{E_{U}}$, where $k_{b} T$ is the thermal energy and $E_{U}$ is the Urbach energy describing the exponential decay of the trap states within the bandgap. ${ }^{285}$ The transient photocurrent is an alternative to spectroscopic methods to investigate subbandgap states. ${ }^{286}$ In HgTe, the Urbach energy ranges from $35 \mathrm{meV}$ to $50 \mathrm{meV}$ depending on the capping ligands. ${ }^{278} \mathrm{~A} \mathrm{low}$ value for the Urbach energy indicates high surface passivation and can minimize the open circuit losses for photovoltaic devices. ${ }^{287}$ Combining this determination of the trap state distribution and photoemission measurements, Martinez et al. proposed a reconstructed spectrum for HgTe NCs, which included the trap state distribution (see Figure 25e). 


\section{LIGHT EMISSION}

Due to their high luminescence in the UV and visible regions, $\mathrm{Cd}$ - and $\mathrm{Zn}$-based chalcogenides were the first and most studied materials under NCs. Mercury chalcogenides were introduced later as promising II-VI narrow bandgap materials for infrared light emission. ${ }^{170}$ The first findings were reported by Rogach et al., who grew HgTe NCs for the first time, ${ }^{20}$ as shown in Figure $26 a$. The authors achieved small HgTe NCs with a tail of absorption in the infrared region. This material was photoluminescent in the nearinfrared region and up to the first telecom region $(1.3 \mu \mathrm{m})$. The interest in such near-infrared emitters was, at first, motivated by bioimaging, ${ }^{116,129}$ since the near-infrared window overlaps with the low absorption spectral range of water (and consequently of biological tissues). Light emission was not extensively investigated at the device level for mercury chalcogenides. ${ }^{23,288,289}$ This drawback was not the result of a material limitation but rather due to the difficulty of introducing $\mathrm{Hg}$-based materials for lighting due to their toxicity. A significant part of the work related to the HgX NCs' PL relates to its use as a tool to examine nonradiative channels. This aspect is used to understand the fundamental limits of NCs occurring through Auger relaxation or coupling to the organic ligands.

This chapter is divided in two main parts. The first part is related to the photoluminescence in HgX NCs. Intraband luminescence and reports for stimulated emission are discussed in this section. The second part is dedicated to electroluminescence in HgX based devices.

\subsection{Photoluminescence}

\subsubsection{Interband luminescence}

Following the work of Rogach et al. ${ }^{20,115,290}$ many researchers attempted to increase the emission wavelength. This effort was strongly correlated with synthetic developments. Among the key developments, Kovalenko et al. reported an emission up to $\approx 4$ $\mu \mathrm{m}$ (see Figure 26b) that was extended to $5 \mu \mathrm{m}$ (Figure 26c) and $8 \mu \mathrm{m}$ by adding a regrowth step. ${ }^{28}$ While further progress was made to strongly redshift the absorption ${ }^{29,30}, 8 \mu \mathrm{m}$ remains the reddest value ever reported for the PL of NCs.

The difficulty of measuring redder PL signals is twofold: the lack of an easily available commercial setup and high cost of detectors in the MWIR/LWIR ranges and the intrinsic issue limiting the PLQY of infrared NCs. As depicted in Figure 26d and reported in Table 4, the PLQY rapidly decreases with wavelength. For example, a reasonable PLQY can still be obtained at $2 \mu \mathrm{m}$ when performing aqueous synthesis. Rogach and coworkers pushed the PLQY up to $17 \%$ for an emission of approximately $2 \mu \mathrm{m} .{ }^{101}$ However, at 5 $\mu \mathrm{m}$, the PL efficiency is only a few $0.01 \%$. This drop is caused by the combination of two effects. If we neglect the nonemitting (dark) NCs, ${ }^{291}$ then the PLQY can be expressed as $Q Y=\frac{\Gamma_{r}}{\Gamma_{r}+\Gamma_{n r}}$, where $\Gamma_{r}$ is the radiative emission rate and $\Gamma_{n r}$ is the nonradiative decay rate, accounting for all the possible loss mechanisms (Auger, phonon, energy transfer, etc.). The radiative lifetime can be expressed $\mathrm{as}^{292} \Gamma_{r}=\frac{\omega^{3}}{3 \hbar \mathrm{c}^{3}} \mu^{2}$, where $\omega$ is the frequency of emission, $\hbar$ is the reduced Planck constant, $c$ is the light velocity and $\mu$ is the dipole associated with the transition. Van Driel et al. reported a different scaling for the radiative decay rate, with the emission frequency presenting a linear dependence. ${ }^{293}$ Nevertheless, both the modeling approaches for the radiative decay rate indicated a lower rate as the emission shifted toward longer wavelengths. Thus, if the nonradiative decay rate is considered to be a result of the surface chemistry, then the slowing of the radiative component in the infrared region is primarily responsible for the observed QY loss. Specifically, this phenomenon is responsible for one and a half orders of magnitude decay (in the $\omega^{3}$ scaling hypothesis) out of the two and a half orders of magnitude decay of the PLQY observed as the emission wavelength increases from 2 to $5 \mu \mathrm{m}$.

This intrinsic decay cannot be overcome by loss reduction (i.e., decrease in the nonradiative decay rate). To design faster radiative IR emitters, the dipole remains the only degree of freedom. Quantum engineering can facilitate the development of heterostructures in which the wave functions are designed to increase the dipolar moment of the transition. At present, this strategy remains unexplored for mercury chalcogenides. This approach can be implemented "artificially" by tuning the light matter coupling: recently, Sergeev et al. reported the coupling of a HgTe NC film to an array of nanobumps, ${ }^{21}$ as shown in Figure 26 e. This array is used to introduce plasmonic resonance and increase the effective electromagnetic field absorbed by the NCs. The obtained $\mathrm{PL}$ is later enhanced by a factor of 5; see Figure $26 \mathrm{f}$.

Table 4 Figures of merit relative to the PL from $\mathrm{HgX}$ NCs

\begin{tabular}{lllllll}
\hline Material & PL peak $(\mu \mathrm{m})$ & Line width & PLQY (\%) & Life time & Comment & Year/Reference \\
\hline \hline
\end{tabular}




\begin{tabular}{|c|c|c|c|c|c|c|}
\hline HgTe & 1.3 & $\approx 200 \mathrm{~nm}$ & 48 & - & $\begin{array}{l}\text { Synthesis } \\
\text { based on thiol } \\
\text { capping in } \\
\text { aqueous } \\
\text { medium }\end{array}$ & $1999 / 20$ \\
\hline $\mathrm{CdHg}_{1-\mathrm{x}} \mathrm{Te}_{\mathrm{x}}$ & $0.8-1.1$ & $\approx 150-350$ & 40 & - & Size tunability & $2001 / 32$ \\
\hline HgTe & $0.9-2.0$ & $\approx 200-400$ & 50 & & $\begin{array}{l}\text { for NIR } \\
\text { luminescence }\end{array}$ & \\
\hline HgTe & $\begin{array}{l}1.2-3.5 \\
@ 3.5\end{array}$ & $\approx 800$ & $\begin{array}{l}40 \\
0.5-2\end{array}$ & - & $\begin{array}{l}\text { Wide } \\
\text { emission from } \\
\text { the near to } \\
\text { the mid-IR }\end{array}$ & $2006 / 27$ \\
\hline HgTe & $\begin{array}{l}1.3{ }^{5} \\
@ 5\end{array}$ & $\approx 1000 \mathrm{~nm}$ & - & - & $\begin{array}{l}\text { Narrow } \\
\text { luminescence } \\
\text { from near and } \\
\text { mid-IR }\end{array}$ & $2011 / /^{95}$ \\
\hline HgTe & $\begin{array}{l}1.5-5.7 \\
@ 2\end{array}$ & $\approx 300 \mathrm{~nm}$ & $\begin{array}{l}10-10^{-2} \\
2\end{array}$ & $\begin{array}{l}48 \text { ps (Auger) } \\
2 \text { ns (exciton) }\end{array}$ & $\begin{array}{l}\text { PLQY of mid } \\
\text { IR emission }\end{array}$ & $2014 / /^{168}$ \\
\hline HgTe & $\begin{array}{l}1.3-2.3 \\
@ 2.07\end{array}$ & $\approx 700 \mathrm{~nm}$ & 17 & - & $\begin{array}{l}\text { Aprotic based } \\
\text { synthesis }\end{array}$ & $2017 / 101$ \\
\hline HgTe & $1.5-2.7$ & $200-700 \mathrm{~nm}$ & - & - & $\begin{array}{l}\text { Two step } \\
\text { synthesis } \\
\text { using a } \mathrm{H}_{2} \mathrm{Te} \\
\text { gas precursor }\end{array}$ & $2020 / 92$ \\
\hline $\mathrm{HgS}$ & 5 & $\approx 1150 \mathrm{~nm}$ & $10^{-1}-10^{-2}$ & - & Intraband PL & $2014 / /^{33}$ \\
\hline $\mathrm{HgSe} / \mathrm{CdS}$ & 5.08 & $\approx 1150 \mathrm{~nm}$ & $10^{-1}-10^{-2}$ & - & Intraband PL & $2016 /{ }^{169}$ \\
\hline
\end{tabular}

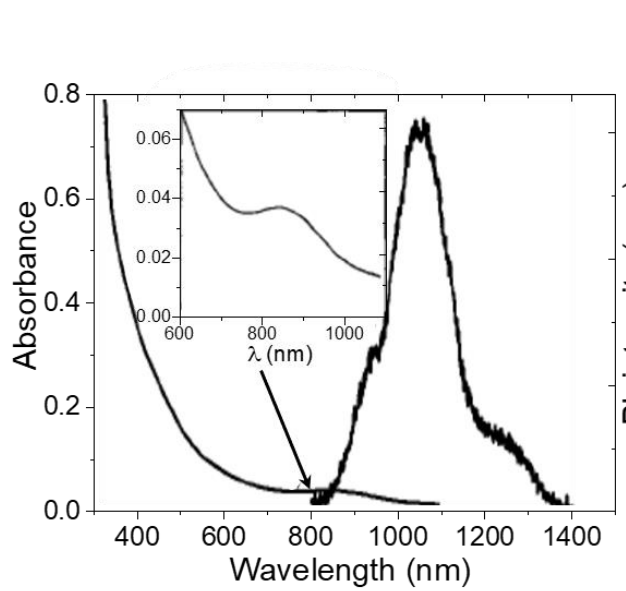

d. Wavelength $(\mathrm{nm})$

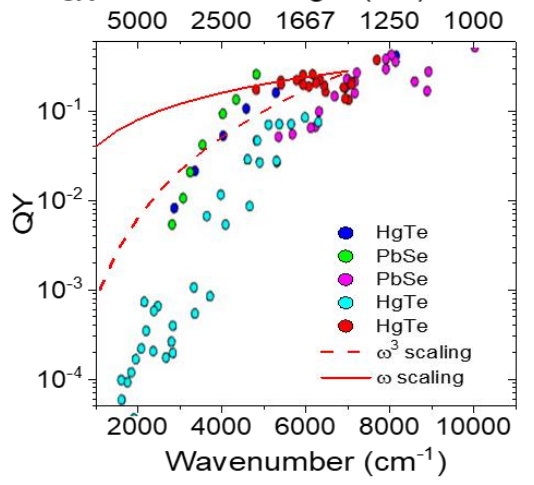

e.

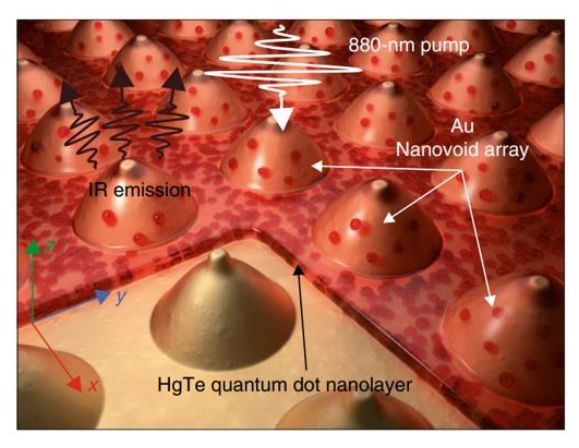

Wavelength $(\mu \mathrm{m})$

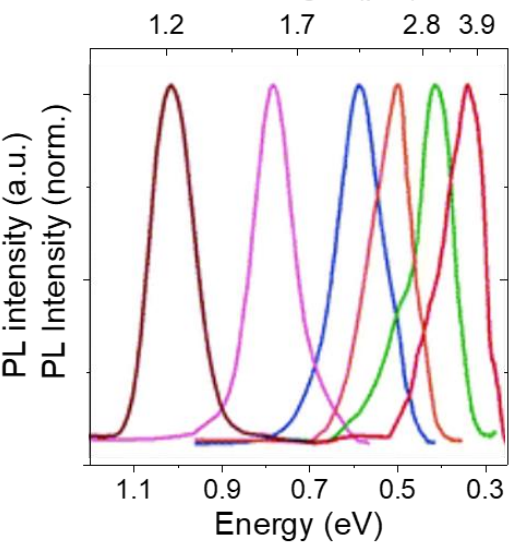

Energy $(\mathrm{eV})$

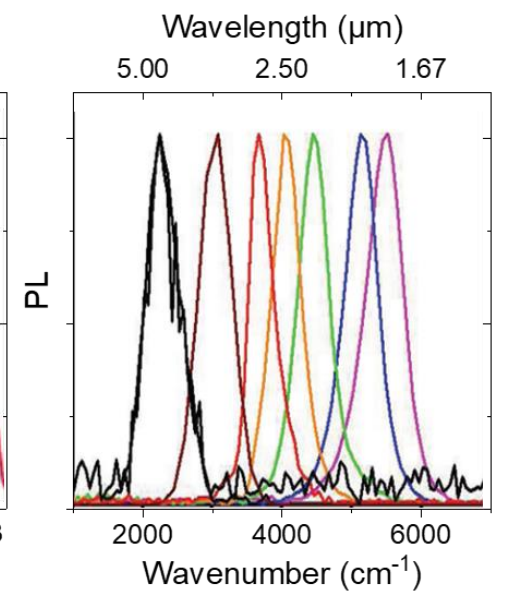

f.

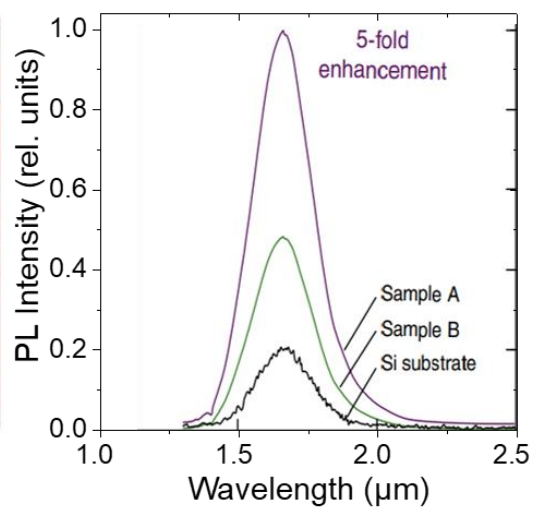

Figure 26 a. Absorption and photoluminescence spectra of HgTe NCs reported by Rogach et al. Adapted from ref ${ }^{32}$. b. Room temperature PL spectra of HgTe NCs reported by Kovalenko et al. Part 
$b$ is adapted with permission from ref ${ }^{27}$. Copyright (2006), American Chemical Society. c. PL spectra of HgTe NCs reported by Keuleyan et al. Part c is adapted with permission from ref ${ }^{95}$. Copyright (2011), American Chemical Society. d. Comparison of the PLQY between HgTe and PbSe with different growth methods: Part $d$ is adapted with permission from ref ${ }^{101}$. Copyright (2017), American Chemical Society. e. Scheme of a HgTe film coated on a laser printed Au nanobump array. $f$. Emission spectra of the HgTe NC layer on a Si substrate or on a laser printed Au nanobump array (part e.). Samples $A$ (purple) and $B$ (green) have different lattice resonances (1480 \pm 30 for $A$ and $1600 \pm 30 \mathrm{~nm}$ for $B$ ). Parts $e$ and $f$ are adapted from ref ${ }^{21}$.

The second limitation to obtain bright IR emitters is also specific to infrared. IR is often seen as part of the electromagnetic spectrum in which chemical bonds present a fingerprint signal: infrared spectroscopy is commonly used as an analytical technique to clarify the chemical composition of a sample. The surface chemistry of NCs leads to a dramatic limitation in regard to the IR emission of NCs because of the strong IR absorption of ligands. At the end of synthesis, NCs are generally capped with ligands that include long alkyl chains. The ligands present a strong IR absorption at $\approx 2900 \mathrm{~cm}^{-1}$ due to the $\mathrm{C}-\mathrm{H}$ bond resonance. The magnitude of this absorption is often useful to determine whether ligand exchange is successful. Due to the proximity of ligands to the inorganic core, a highly efficient energy transfer occurs from the exciton to the organic shell. This process introduces nonradiative losses in the PL and can be attributed to a one order of magnitude drop in the PL efficiency below the radiative limit (see Figure 26d). A possible strategy to overcome this limitation is to use purely inorganic surface chemistry. On $\mathrm{HgX} \mathrm{NCs,} \mathrm{As} \mathrm{S}_{3}$ is, to date, the only fully inorganic ligand that has been grafted onto NC surfaces. ${ }^{35}$ However, no report exists on the potentially beneficial effects of these inorganic ligands for $\mathrm{PL}$, as these ligands were introduced for electron transport enhancement. The introduction of a core/shell with a type I band alignment is also a viable strategy to enhance the PLQY by preventing the wave functions from experiencing surface traps. ${ }^{9,16}$ As discussed in the section dedicated to heterostructure growth presented earlier in this review, the growth of $\mathrm{HgX}$ based heterostructures is highly challenging because $\mathrm{HgX}$ cores are mostly incompatible with the high temperature growth methods required to obtain $\mathrm{CdX}$ shells. Among the few reports dedicated to the shelling on $\mathrm{HgX}$ cores, mostly toward CdX shells, ${ }^{33,136,185,187}$ the introduction of the shells does not lead to a significant PLQY enhancement.

\subsubsection{Intraband luminescence}

While previous discussions have mostly focused on undoped HgX NCs, self doped HgX NCs can also present intraband PL, ${ }^{33,169}$ as shown in Figure 27c. In this case, the PL signal spectrally overlaps with the intraband absorption without the Stokes shift. Depending on the exact filling of the $1 \mathrm{~S}_{\mathrm{e}}$ state, which can be modulated with surface chemistry, dual color luminescence attributable to both the inter and intraband absorption can be obtained. ${ }^{169}$ The PLQY of the intraband transition is similar to that from the interband transition at the same wavelength.
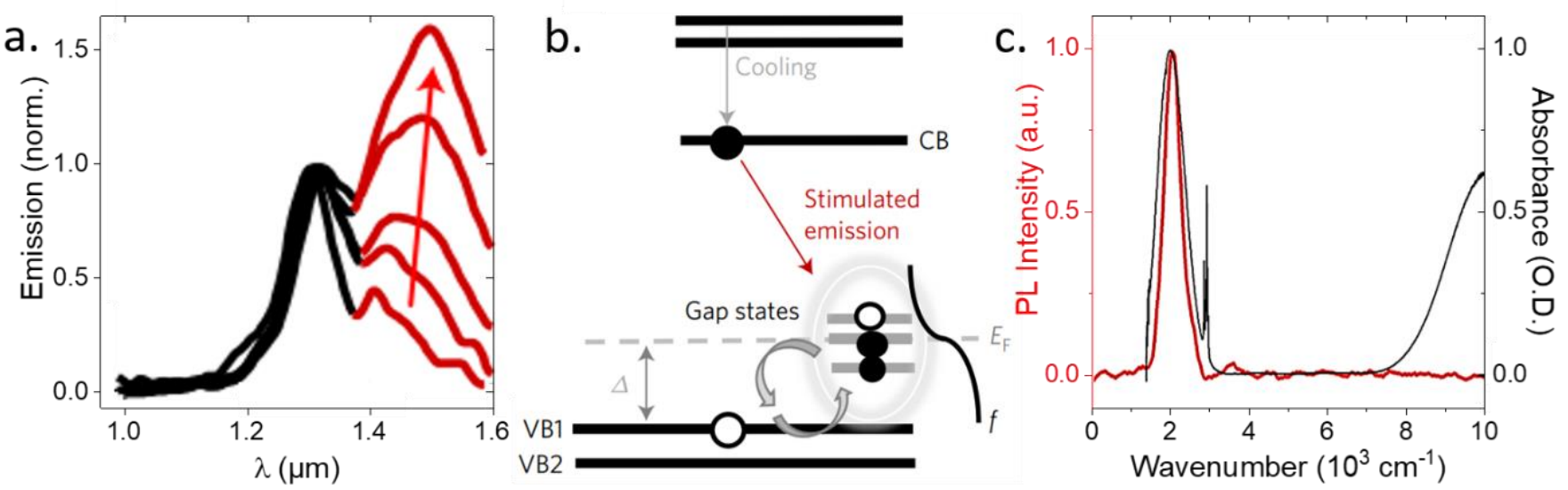

Figure 27 a. Amplified spontaneous emission spectra of a free-standing $\mathrm{HgTe}$ thin film with increasing stripe length (red arrow). b. A 4-level scheme in which the stimulated emission involving an electron from the $C B$ to the empty surface defect levels close to the valence bands (VB $\left.B_{1 / 2}\right)$ does not have any counterpart in absorption, leading to a thresholdless gain. Parts a and $b$ are reprinted by permission from Nature, Nature Materials, ref ${ }^{22}$, [COPYRIGHT] (2018) c. Intraband PL emission spectrum (red) from the $1 S_{e}-1 P_{e}$ transition of ambient $n$-type $\mathrm{HgSNCs}$ after photoexcitation at $808 \mathrm{~nm}$. The absorption spectrum (in black) overlaps with the PL emission spectrum. Part $c$ is adapted with permission from ref 33. Copyright (2014), American Chemical Society. 


\subsubsection{Lasing}

In addition to the $\mathrm{PL}$, lasing of $\mathrm{NCs}^{294}$ has been developed in recent years. ${ }^{295-298}$ To design a cavity, Shopova et al. used $\mathrm{Si}$ microspheres that couple to $\mathrm{Hg}(\mathrm{Cd}) \mathrm{Te} \mathrm{NCs}{ }^{299}$ The authors obtained a lasing signal from 1240 to $1780 \mathrm{~nm}$ with a low pump threshold. ${ }^{300}$ In these early reports, short ( $f s$ and ps) pulsed excitations were considered to generate a transient gain at times smaller than the Auger lifetime. Nevertheless, the long term goal is to demonstrate the steady state or quasi (ns) steady state gain to enable broad band light amplification at a telecommunication bandwidth ( $\mathrm{GHz}$ and more). A step in this direction was reported recently by Geiregat et al. ${ }^{22}$ The authors realized stimulated emission with a low threshold $\left(40 \mathrm{~mW}^{\mathrm{cm}}{ }^{-2}\right)$ in small $\mathrm{HgTe} \mathrm{NCs}(\mathrm{see}$ Figure 27a). Such a low level of excitation is an asset since it is compatible with sunlight excitation or low current density operation of LEDs $\left(20 \mathrm{~mA} \cdot \mathrm{cm}^{-2}\right)$. The authors succeeded in demonstrating a gain using a nanosecond pulsed laser, which is a critical step toward developing electrically driven laser diodes. The authors attributed the origin of the long life gain to the presence of surface traps in the vicinity of the valence band (see Figure 27b). Such traps likely contribute to the formation of a four-level system enabling a long-lived population inversion. These results must be correlated with future developments of HgTe NC-based LEDs. 

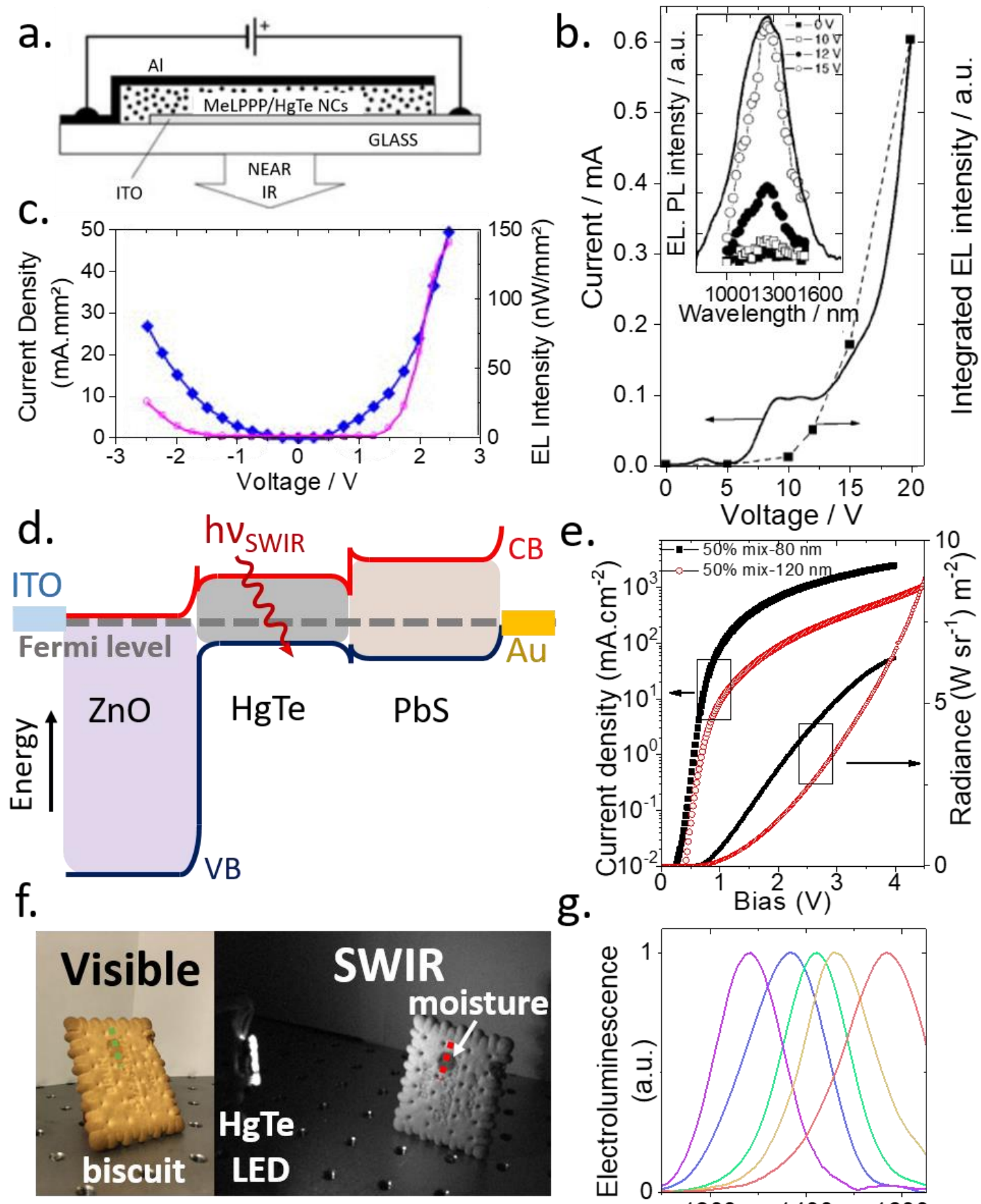

e.

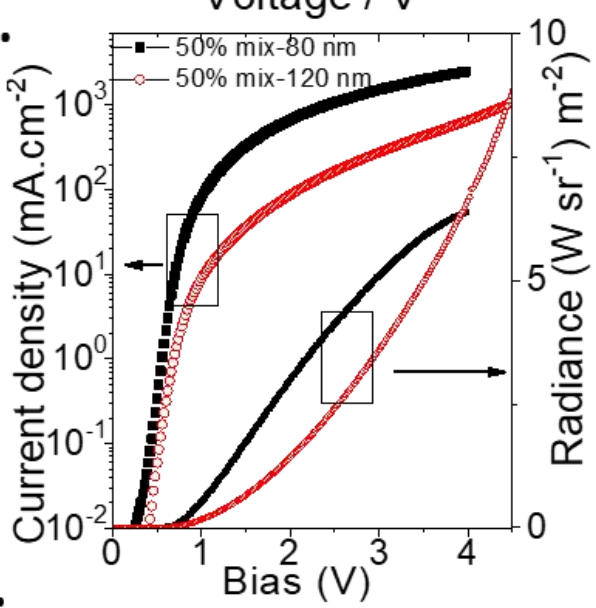

g.

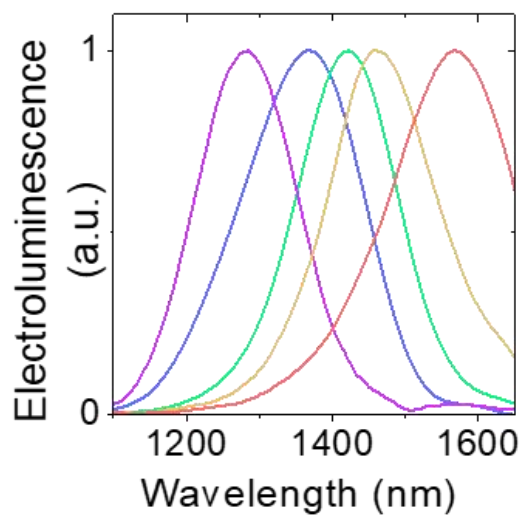

Figure 28 a. Representation of the MeLPPP/HgTe NC device; ITO: indium tin oxide, NIR light: near infrared light. b. Current-voltage (-) and integrated electroluminescence (EL) intensity-voltage (squares connected by a dashed line) traces of the MeLPPP/HgTe NC device. The inset shows the electroluminescence spectra of the MeLPPP/HgTe NC device obtained at different voltages in comparison with the photoluminescence spectrum of $\mathrm{HgTe}$ NCs in a composite MeLPPP/HgTe film. Parts $a$ and $b$ are reprinted with permission from ref ${ }^{288}$. Copyright (C) 2004 WILEY-VCH Verlag GmbH \& Co. KGaA, Weinheim. c. Typical I/V/L characteristics of an NIR emissive HgTe quantum dot device (ITO/PEDOT/HgTe/Al). Blue solid diamonds: current; pink open circles: NIR luminance. Part $c$ is reprinted from ref ${ }^{289}$, with permission from AIP Publishing. $d$. Energy band profile for a diode made of ITO/ZnO/HgTe/PbS/Au. e. Current and radiance as a function of the applied bias for two thicknesses of the emitting layer and with a 50:50 volume ratio of $\mathrm{HgTe}$ and the $\mathrm{ZnO}$ solution. $\mathrm{f}$. Visible (a) and 

obtained from HgTe NCs. Parts $d-g$ are adapted from ref ${ }^{23}$.

The efforts dedicated to achieving electroluminescence (EL) using mercury chalcogenides are extremely few compared to those for PbS NCs, which is likely correlated to the lower amount of work dedicated to the development of vertical geometry photovoltaic devices based on HgTe ${ }^{46,48,146,192,301-304}$ compared with PbS. In addition, HgS and HgSe are self doped and present midinfrared intraband transitions (see the previous section dedicated to intraband luminescence) owing to which the device integration is relatively challenging due to the low PL efficiency. ${ }^{33,169}$ A first structure was proposed by Kotysh et al., ${ }^{288}$ which involved HgTe NCs mixed into a conjugated polymer to form a blend structure. The latter was surrounded by Al and ITO contacts (see Figure 28a). EL at $1300 \mathrm{~nm}$ was observed; however, the turn on voltage was high, at approximately $10 \mathrm{~V}$ (i.e., 10 times the bandgap energy, see Figure 28b), suggesting an unoptimized structure. Later, O'Connor et al. ${ }^{289}$ proposed a new structure based on an ITO/PEDOT:PSS/HgTe/Al stack. This ITO:PEDOT combination is extensively used as a hole injection layer for OLEDs and visible quantum dot-based LEDs. Compared to wider bandgap materials, the narrow bandgap of HgTe reduces the need for a second (or even third) hole transport layer to make the HOMO potential resonant with the emitting layer valence band. The operating bias is significantly reduced, and the turn on voltage is below $2 \mathrm{~V}$ when using this structure (see Figure 28c). The later remains far above the bandgap. Since this research, the electronic structure and doping of the materials have undergone considerable progress. HgTe NCs with emission in the near-IR region exhibit a strong $p$-type nature. The aluminum top contact is easily oxidized into insulating alumina, which leads to an insufficient electron injection. After this demonstration, there was a 15 y period without any report dedicated to EL based on HgTe NCs. Recently, Qu et al. ${ }^{23}$ proposed a structure inspired by PbS NC solar cells. ${ }^{305,306}$ The authors used a ZnO layer and an EDT capped layer of PbS for the electron and hole transport layers, respectively (see Figure 28d). The emitting layer was made of a $\mathrm{ZnO}: \mathrm{HgTe}$ mixture. The authors found that the optimal $\mathrm{ZnO}$ to $\mathrm{HgTe}$ ratio was approximately 1:1. This ratio facilitates the formation of an ambipolar bulk heterojunction while controlling the charge injection by modulating the amount of $\mathrm{ZnO}$ as a hole blocking layer. With this structure, a subband gap (0.6 V, see Figure $28 \mathrm{e})$ turn on voltage is achieved. The authors demonstrated the development of a bright LED with a maximum EQE of $0.7 \%$, stable emission over $30 \mathrm{~h}$ of continuous operation in air without any LED encapsulation, and spectral tunability from 1250 and $1600 \mathrm{~nm}$ (Figure 28g). The authors finally used the bright LED as an SWIR light source for active imaging applications (see Figure 28f). The authors also indicated that moisture detection could be easily realized using such near IR emitting LEDs. One unaddressed question regarding HgX NC LEDs is light extraction. Due to an optical index mismatch, approximately $80 \%$ of the generated photons are waveguided into the substrate. Various strategies have been reported to obtain direct emission from HgTe NCs, including the use of Bragg mirrors ${ }^{307}$ or coupling HgTe NCs to a photonic crystal. ${ }^{308}$ 


\section{ELECTRONIC TRANSPORT}

This section addresses the electronic transport in $\mathrm{HgX} \mathrm{NCs,} \mathrm{mostly} \mathrm{in} \mathrm{the} \mathrm{array} \mathrm{form.} \mathrm{This} \mathrm{review} \mathrm{is} \mathrm{focused} \mathrm{on} \mathrm{dark} \mathrm{conduction}$ since the next section is dedicated to photoconduction and detection applications of HgX NCs. The chapter is organized as follows. We first discuss the specificity of the electronic conduction in a range of carrier densities $\left(10^{-3}-1\right.$ carriers per NC), which is considerably higher than that typically observed for wide bandgap semiconductors but considerably lower than that observed for metals ( 1 electron per atom). Next, we discuss the effect of the surface chemistry on the transport and stability of the material's electrical properties under atmospheric conditions. Finally, a large part of the discussion is dedicated to the design of field effect transistors and their use in examining electronic transport.
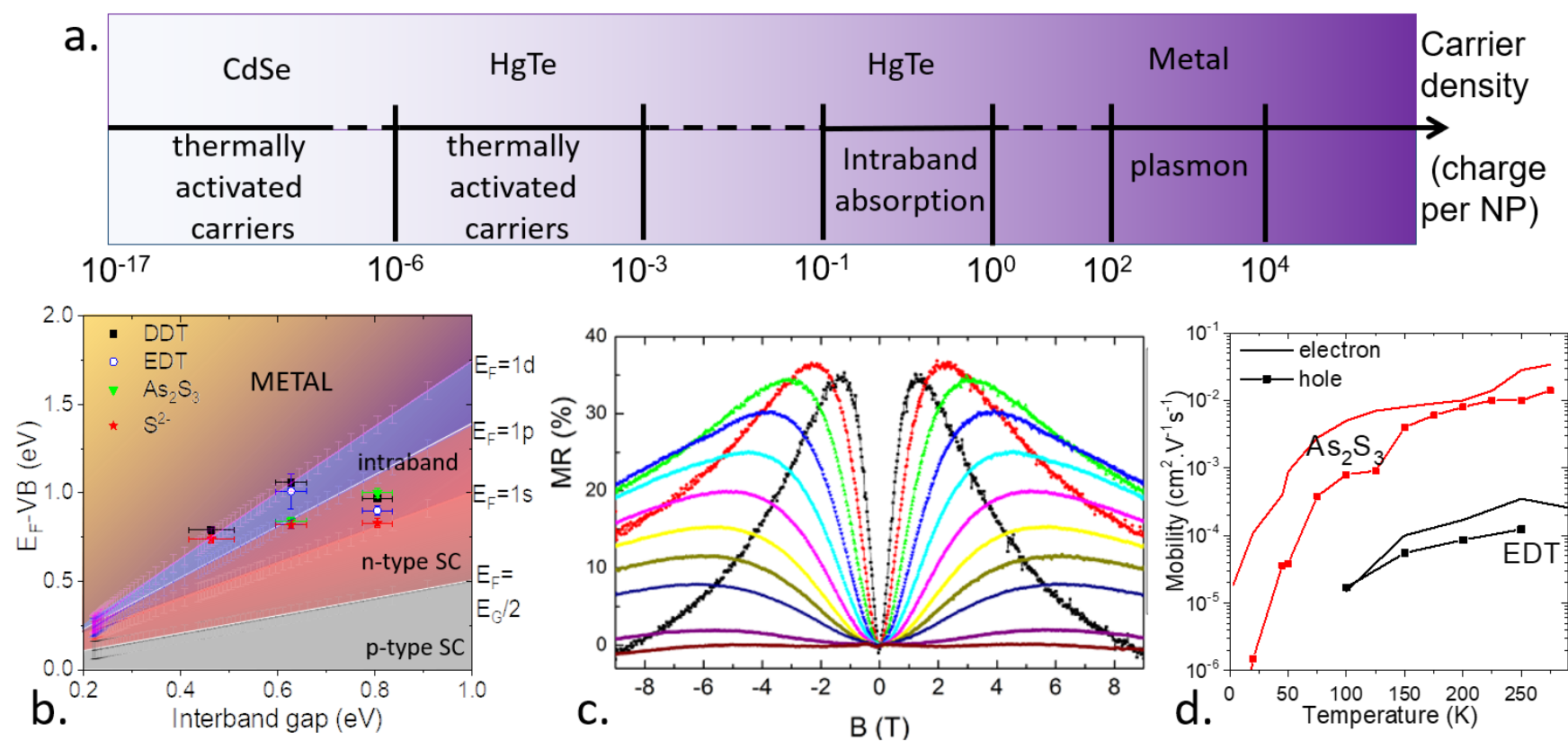

Figure 29 a. Scale illustrating doping in various NCs. b. Fermi level energy with respect to the valence band as a function of the interband gap for HgSe NCs. The solid lines correspond to the mid-interband gap and the $1 S_{e}, 1 P_{e}$ and $1 D_{e}$ states from the 3-band $k \cdot p$ simulation. Part $b$ is adapted with permission from ref ${ }^{122}$ Copyright (2017), American Chemical Society. c. Magnetoresistance as a function of the applied magnetic field for heavily Mn-doped HgS (15\%) NCs. Part c is adapted with permission from ref ${ }^{206}$ Copyright (2015), American Chemical Society. d. Electron and hole mobilities of a HgTe CQD film processed with $A s_{2} S_{3}$ and EDT as a function of temperature. Part $d$ is reprinted with permission from ref ${ }^{35}$. Copyright (C) 2013 WILEY-VCH Verlag GmbH \& Co. KGaA, Weinheim.

As discussed earlier, a key specificity of HgX NCs with respect to other NCs is their large carrier density. Figure 29a summarizes the main results discussed in the section dedicated to doping. The figure provides a sense of scale for the expected carrier densities in various NCs. The large carrier density originates from a combination of the low carrier activation energy of a narrow bandgap semiconductor and self doping that can lead to high doping levels of up to several carriers per NC. In a simple hydrodynamic model, the current density scales as the product of the carrier density and film mobility. For a given surface chemistry (i.e., for a given mobility), this aspect can lead to a significantly dark conductance for a film of HgTe with approximately 0.01 carriers per NC, especially compared to a CdSe film that has virtually no thermally activated carriers. This increased conductance provides the opportunity to examine the transport in an FET configuration, although this increase impedes IR detection. The large dark carrier density reduces the photocurrent-induced modulation and requires cryogenic conditions to achieve optimal performance. Martinez et al. exploited the doped nature of HgSe NCs to investigate the effect of confinement on the metal-insulator transition. ${ }^{122}$ Doping is an intuitive key to examine the metal-insulator transition. ${ }^{309,310}$ According to the Mott criterion, metallic behavior should occur if $a_{0} n^{1 / 3}>0.25,{ }^{311}$ where $n$ is the carrier density and $a_{0}=\frac{h^{2} \varepsilon_{0} \varepsilon_{r}}{\pi m^{*} e^{2}}$ is the Bohr radius with $h$ being the Planck constant, $\varepsilon_{0}$ being the vacuum permittivity, $\varepsilon_{r}$ being the material dielectric constant, $m^{*}$ being the effective mass and $e$ being the elementary charge. This approach has been used to induce a metallic nature in doped Si NCs. ${ }^{309}$ In HgSe, because of self doping, confinement influences the relative position of the bands with respect to the Fermi level, as shown in the phase diagram in Figure $29 \mathrm{~b}$. The authors indicated that by increasing the size (i.e., reducing confinement), the 1S, $1 \mathrm{P}$, and finally $1 \mathrm{D}$ states can be filled 
and, consequently, the carrier density is increased to 2, 8 and 18 electrons per NC, respectively. The authors, through temperaturedependent conductance measurements, demonstrated that the transport law driving the current in HgSe arrays is affected by the state filling at the NC level. At low carrier densities, the results obtained using Mott's law $\left(\sigma \propto \exp \left(-\left(T_{M} / T\right)^{1 / 4}\right)\right.$, where $\sigma$ is the conductance and $\mathrm{T}$ is the temperature, match well with the experimental results. This aspect reflects that the density of states drives the current magnitude at low carrier densities. At high carrier densities, typically when the HgSe 1D state is filled, the number of electrons increases and a more metallic behavior is expected. The conductance temperature dependence in this range can be defined using Efros-Shklovskii's law $\left(\sigma \propto \exp \left(-\left(T_{E S} / T\right)^{1 / 2}\right)\right.$ ). This law is the conventional law observed in an array of metallic nanoparticles. ${ }^{312}$ When the $D$ states are filled, the density of the states increases and the availability of the empty states no longer drives the current magnitude. At this point, the current is determined by the Coulombic cost of the nanoparticle charging. This aspect shows that at the nanoparticle level, the NC switches from a semiconductor behavior to a metallic behavior. ${ }^{122}$ However, owing to the weak coupling between the individual nanoparticles at the film level, the collective behavior (i.e., the film behavior) is similar to that of a semiconductor, with an overall increase in the electrical resistance upon cooling.

\subsection{Surface chemistries for transport in the $\mathrm{HgX}$ array}

In NC arrays, transport is driven by the surface chemistry, which determines the carrier mobility. ${ }^{24,313}$ However, in early studies involving transport in arrays of $\mathrm{HgX} \mathrm{NCs,} \mathrm{ligand} \mathrm{exchange} \mathrm{procedures} \mathrm{were} \mathrm{not} \mathrm{as} \mathrm{developed} \mathrm{as} \mathrm{they} \mathrm{are} \mathrm{today,} \mathrm{and} \mathrm{sintering} \mathrm{was}$ the classic strategy to enhance the transport properties. The latter processes can be implemented through the aggregation of particles during synthesis ${ }^{3}$ or induced by mild temperature annealing $\left(150{ }^{\circ} \mathrm{C}\right) .{ }^{194,314}$ Annealing at temperatures above the growth temperature leads to a strong particle sintering, which favors the nanoparticle coupling associated with a higher carrier mobility. However, this strategy is not viable to design IR sensors since aggregation considerably broadens the optical features of the NCs, leading to an inferiorly defined cutoff wavelength and delocalized oscillator strength. The material obtained from this approach presents $p$-type conduction with hole mobility of the order of $1 \mathrm{~cm}^{2} \mathrm{~V}^{-1} \mathrm{~s}^{-1}$. This $\mathrm{p}$-type nature may be the result of material oxidation occurring during annealing or may result from the expected $p$-type nature of small HgTe NCs even without annealing. ${ }^{314,315}$ Moreover, a low film quality was achieved from aggregated NCs. ${ }^{3}$ Although electronic coupling within a grain can be large, it remains weak among aggregates. Moreover, the surface of the film obtained from the aggregated NCs is extremely rough, which explains why all the early reports related to $\mathrm{HgX}$ for IR detection were based on planar geometry devices for which high quality films are not required.

To achieve milder conditions to obtain conductive films of $\mathrm{HgX} \mathrm{NCs,} \mathrm{solid} \mathrm{state} \mathrm{ligand} \mathrm{exchange} \mathrm{with} \mathrm{various} \mathrm{short} \mathrm{organic} \mathrm{ligands}$ was examined. The library of reported ligands includes short amines ${ }^{266}$ and thiols ${ }^{278}$ due to the strong affinity of mercury atoms for sulfide ions. During this procedure, the film is exposed to a dilute solution of short ligands in a nonsolvent, commonly an alcohol. The concentration of ligands used during this procedure differs from group to group but is typically approximately $1 \%$ in mass. This concentration is critical to obtain sufficient ligand exchange: when the value is extremely low, the exchange is not complete. When the ligand concentration is extremely high, the film tends to peel off from the substrate. As ligand exchange occurs, the volume of the particle is reduced. For example, on a $5 \mathrm{~nm}$ particle initially capped with $C_{12}$ chains ( $\approx 1.5 \mathrm{~nm}$ ) exchanged for ethanedithiol (EDT), the volume reduction reaches a factor of 2.4. This phenomenon leads to a strong volume contraction of the film, which induces crack formation at the mesoscopic scale. Consequently, multilayer deposition must be implemented for the following two reasons:

(i) due to diffusion, ligand exchange only occurs on a thin slab (i.e., ligand exchange on thick films is not complete) and (ii) the cracks formed during the previous steps must be filled. When implementing solid state ligand exchange, the band edge feature is more preserved than that in the case of particle sintering; however, the carrier mobility is considerably reduced, ranging from $10^{-}$ ${ }^{4}$ to $10^{-3} \mathrm{~cm}^{2} \mathrm{~V}^{-1} \mathrm{~s}^{-1}$. However, this approach is limited in terms of the achievable film thicknesses, as the overall film quality degrades when too many layers are deposited. The procedure was later developed by Guyot-Sionnest and coworkers, who reported EDT ligand exchange under acidic conditions. ${ }^{301,303}$ The mixture used for ligand exchange was composed of EDT and $\mathrm{HCl}$ diluted in ethanol. The acid protonates the long thiolate grafted at the surface, which eases its removal from the NC surface, while chloride ions likely contribute to electronic surface passivation. More recently, the combination of thiol and chloride was further updated for a combination of $\mathrm{HgCl}_{2}$ and mercaptoethanol. Using this approach, the carrier mobility could be expanded to more than $1 \mathrm{~cm}^{2}$ $\mathrm{V}^{-1} \mathrm{~s}^{-1}$ for both $\mathrm{HgTe}^{36,316}$ and HgSe. ${ }^{317}$ This increased carrier mobility was also observed by other groups, ${ }^{146,318}$ although values above $1 \mathrm{~cm}^{2} \mathrm{~V}^{-1} \mathrm{~s}^{-1}$ were not achieved. The discrepancy could likely be attributed to the different shapes of HgTe nanoparticles used by the different teams (spheres vs. branched particles), which may impact the particle packing and thus the interparticle coupling. This approach is compatible with liquid phase ligand exchange, allowing the formation of concentrated solutions of $\mathrm{HgTe}$ nanoparticles. During this procedure, the NCs initially dispersed in a nonpolar solvent are mixed with a polar phase containing short ionic ligands. ${ }^{24}$ Native long ligands (typically, DDTs) are replaced by short ligands. Owing to this ionic surface, the NCs become soluble in the polar phase. These inks (NCs capped with short ligands compatible with transport and dispersed in a polar solvent) 
can be used for the deposition of thick (> $500 \mathrm{~nm}$ ) layers, which considerably increases the absorption of the film compared with that in the previous generation of ligand exchange procedures. In addition, this dual ligand method leads to increased operation stability in air, with a weak increase in the dark current and monotonic I-T curves.

For infrared nanocrystals, energy transfer from the exciton to ligand absorption is often seen as a key limitation. Consequently, fully inorganic ligands have been investigated. The $\mathrm{As}_{2} \mathrm{~S}_{3}$ material has attracted the most interest, despite its toxicity. Initially, the $\mathrm{As}_{2} \mathrm{~S}_{3}$-based surface chemistry was introduced by Talapin and coworkers as an inorganic strategy to obtain bright near-infrared PbS NCs. ${ }^{319}$ This strategy was later used to build PbS NC arrays with strong photoconduction. ${ }^{320}$ The method can also be applied for $\mathrm{HgX} \mathrm{NCs:} \mathrm{in} \mathrm{this} \mathrm{case,} \mathrm{As}_{2} \mathrm{~S}_{3}$ powder is first dissolved in a short amine, such as propylamine or butylamine. If a $\mathrm{HgX} \mathrm{NC}$ film is directly exposed to this solution, then the film dissolves, and thus, this ligand solution must be diluted in ethanol before conducting a typical ligand exchange. This method has been applied to $\mathrm{HgTe}^{35,42,45}$ and $\mathrm{HgSe} .{ }^{29,122}$ Compared to conventional ligand exchange with short organic thiols, $\mathrm{As}_{2} \mathrm{~S}_{3}$-based ligands lead to increased mobility, as shown in Figure $29 \mathrm{~d}$, typically by two orders of magnitude. The enhanced stability leads to monotonic I-T curves, even for air processing.

Furthermore, atomic layer deposition (ALD) has been explored to realize inorganic infilling among $\mathrm{NCs}^{321,322}$ and develop conducting films. ${ }^{323,324}$ Alumina ${ }^{315}$ and $\mathrm{CdS}^{325}$ deposition are performed on HgTe NC films. A clear reduction in film resistance is observed compared to that before ALD. However, this method suffers from two key limitations. First, the approach is generally conducted at a high temperature (i.e., at approximately or above the NC growth temperature), which leads to sintering, and thus, the drop in resistance is presumably a convolution of the mobility increase and bandgap reduction. Second, the photoresponse is reduced after ALD deposition. ${ }^{325}$ More work is required to clarify the potential of ALD as a strategy to passivate HgX NC films.

\subsection{Magnetotransport}

Transport measurement is relatively straightforward in HgX NC arrays, and the potential of these materials for spintronics has also been investigated. Liu et al. ${ }^{266}$ compared the magnetotransport in $\mathrm{n}$ - and p-type doped HgTe NCs. The field-resolved conductance $(M R)$ presented the first positive magnetoresistance $(M R)$ at a low field $(B<0.2 T)$, resulting from the spin blockade. When a larger magnetic field was applied, the wave function was compressed, leading to a negative MR signal. This second effect strongly depends on the carrier mass. In HgTe, the hole band is barely dispersed (i.e., the hole mass is similar to the free electron mass), and the wave function compression is limited. However, the conduction band has a low mass, owing to which the MR signal is considerably larger than the hole band, reaching an MR of $-10 \%$ under $4 \mathrm{~T}$. To enhance the MR signal, the same team proposed doping NCs (HgS) with a Mn dopant. ${ }^{206}$ For doping levels below $1 \%$, the authors observed a behavior similar to that previously reported for pristine HgTe CQD films; see the bottom lines of Figure 29c. At higher doping, the authors observe a nonmonotonic magnetoresistance for HgS CQDs. At a low field, a strong positive magnetoresistance of up to $35 \%$ is observed, ten times higher than the spin blockade induced positive MR signal observed in undoped NCs. This strong positive MR signal can be attributed to an electron magnetic polaron. At higher fields, the polaron binding energy is reduced, leading to a reduced hopping activation energy and a decrease in resistance. Nevertheless, the observed effect remains small. In comparison, it can be considered that the $\mathrm{Fe} / \mathrm{Cr}$ layers ${ }^{326}$ lead to a modulation of more than $50 \%$ under a similar magnetic field, and tunnel magnetoresistance can lead to a similar resistance modulation with a 100 times lower field. In this sense, the magnetoresistance in an array of HgX NCs is a promising technique to examine wave function localization.

Jeong et al. ${ }^{118}$ studied the transition from diamagnetic to superparamagnetic characteristics in self-doped HgSe NCs as the carrier density increased and filled the 1 Se state. The authors estimated the g-factor to be slightly above 2, and the EPR measurement in a solid state sample yielded a value of 2.09 .

\subsection{Stability and air operation}

A key concern for detector integration relates to the stability of the transport properties, especially when air operation is targeted. As stated previously, transport represents a sensitive approach to identify material modifications. For example, transport properties are usually considerably affected by the formation of an oxide shell or grafting of molecules on the NC surface.

The solid state ligand exchange conducted on the HgTe NC array enhances not only the material conductivity but also the air sensitivity. The latter phenomenon manifests as two distinct effects. First, a continuous rise in dark conductance is observed over time; $^{302}$ see Figure $30 \mathrm{a}$. This increase in dark conductance can reach a factor of 100 after $10 \mathrm{~d}$. The second effect is the nonmonotonic behavior of the temperature dependence of the dark current; ${ }^{35}$ see Figure $30 \mathrm{~b}$. These two effects are particularly problematic for detection applications. The slow rise of the dark current requires regular updates of the correction table used in cameras, and the signal-to-noise ratio of the detector is degraded. Owing to the second effect, cooling, which is the most common strategy to enhance the performance of IR sensors, is rendered ineffective. The origin of this increased dark current remains 
unexplained. A simple strategy to avoid this dark current increase involves air-free processing of the sample (see Figure 30b). Alternatively, Cryer et al. exploited material oxidation. ${ }^{327}$ The authors observed that after a certain time, the I-V curves strongly deviated from ohmic behavior and exhibited hysteretic shapes (see Figure 30d). The authors considered this nonlinear I-V curve to design a memistor. To enable air operation while maintaining the initial transport properties, film encapsulation has been considered. However, this aspect remains one of the main challenges to be addressed by HgX NCs in the coming years. Most encapsulation methods, such as those developed for organic electronics, require high temperature annealing, which leads to detrimental sintering in $\mathrm{HgX}$ arrays. Jagtap et al. showed that any annealing step above $60{ }^{\circ} \mathrm{C}$ leads to a clear redshift of the absorption spectrum and increase in dark conductance. ${ }^{302}$ In addition, the growth of core-shell heterostructures is not a viable alternative to enhance the temperature stability. To overcome these difficulties, the authors used a stack of polymer layers deposited at room temperature (see the inset in Figure 30a). The authors combined water repelling layers (PMMA, PVDF) with an oxygen repelling layer (PVA). The thickness of the stack was approximately $1 \mu \mathrm{m}$, sufficiently large to avoid water and oxygen diffusion but sufficiently small to avoid excessive shortwave infrared absorption. Using this approach, air operation with a quasiconstant dark current was obtained over a three-month period. Later, Talapin and coworkers demonstrated that all these layers were not required and that stable detection properties could be realized using only the PMMA capping layer (see Figure 30c), which simplifies the encapsulation process. ${ }^{36}$

In addition to device encapsulation, significant progress has been made in building more stable films owing to the enhanced film passivation. The use of $\mathrm{As}_{2} \mathrm{~S}_{3}$-based surface chemistry was initially motivated by the identification of a capping ligand enabling air processing of the film without observing nonmonotonic I-T curves. ${ }^{35} \mathrm{As}_{2} \mathrm{~S}_{3}$ leads to improved air stability (i.e., monotonic I-T curve even when using air processing); however, the optimal condition is obtained under air free conditions. More recently, the introduction of the dual ligand strategy (thiol + chloride) has facilitated the limitation of postfabrication air oxidation. ${ }^{146}$
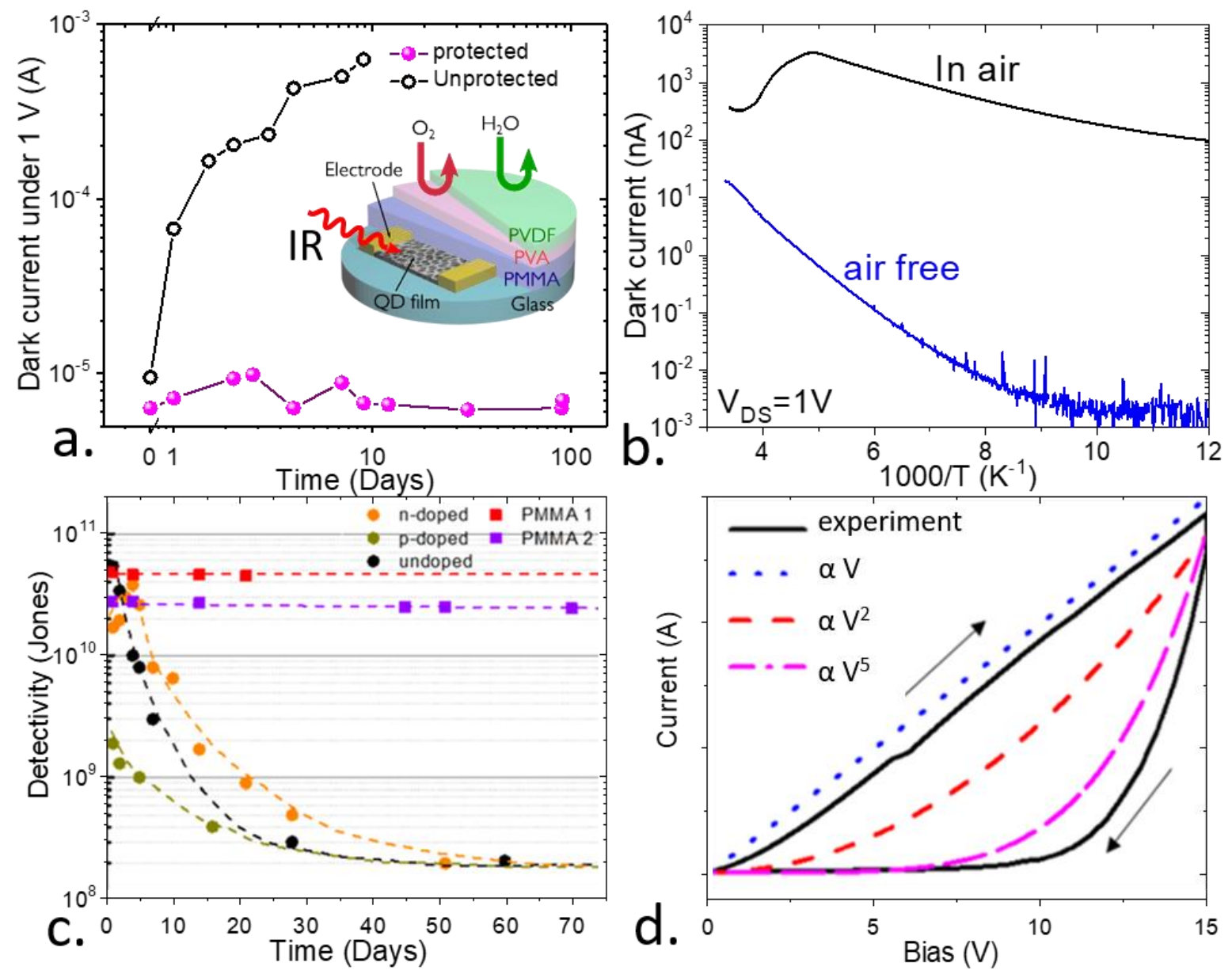

Figure 30 a. Dark current as a function of time spent in air for a HgTe CQD film with and without protective layers. Time zero corresponds to the sample in the air free glove box. The inset is a scheme of the HgTe CQD film device with polymer protective layers. Part a is adapted with permission from ref 302 Copyright (2018), American Chemical Society. b. Current as a function of temperature for a film of $\mathrm{HgTe} C Q D$ s processed with ethanedithiol under air (black) and air free (blue) conditions. Part $b$ is reprinted with permission from ref ${ }^{35}$. Copyright (C) 2013 WILEY-VCH Verlag GmbH \& Co. KGaA, Weinheim c. Detectivity of photoconductive devices using a $\mathrm{HgTe} / \mathrm{hyb}$ rid with and without 
encapsulation. Part $c$ is adapted with permission from ref ${ }^{36}$ Copyright (2019), American Chemical Society. d. I-V under illumination $(2.5 \mathrm{~mW})$ at $300 \mathrm{~K}$ hysteresis; the forward sweep exhibits linear and high carrier density; the backward sweep corresponds to diffusion. Part $d$ is adapted with permission from ref ${ }^{327}$ Copyright (2018), American Chemical Society.

\subsection{HgX Field effect transistors}

Transport is one of the most sensitive measurements for material investigation: while techniques such as EDX, photoemission, or ICP-MS have resolutions of $0.1 \%$ at best, transport is highly sensitive to even small variations (ppm) in composition. In solid state physics, several experimental reports have been presented on transport to estimate the carrier density and mobility. The Hall effect is the most common strategy for evaluating these quantities in bulk materials. In an NC array, Hall measurements are historically not as popular because of the low mobility of the films. Only recently, with the emergence of high mobility films $(\mu>$ $1 \mathrm{~cm}^{2} \mathrm{~V}^{-1} \mathrm{~s}^{-1}$ ), have Hall measurements with reasonable signal-to-noise ratios been made possible;, ${ }^{25,328}$ in 2020 Hall measurements were reported for $\mathrm{HgTe}^{316}$ and $\mathrm{HgSe}^{317}$ as the authors used this characterization to extract the film mobilities and confirm the temperature dependence of the field effect mobility.

To date, field effect transistors (FETs) remain the most commonly used strategy to examine transport in HgX NC arrays while extracting information regarding the nature of the carriers. The large inherent doping of HgX NCs affects the FET properties more than that for other NC materials (CdSe, PbS(e)). For wide bandgap materials, the off state is usually limited by FET leakage. This aspect is generally not the case for HgX NCs, for which the off current is limited by thermally activated carriers. ${ }^{35,316}$ This aspect reduces the current modulation in $\mathrm{HgX} \mathrm{NC}$-based FETs, typically below $10^{3}$ at room temperature, and the value is even lower for self-doped NCs.

Different gating strategies have been used to design $\mathrm{HgX} \mathrm{NC}$-based FETs (see Table 5). $\mathrm{Si}_{\mathrm{S}} \mathrm{SiO}_{2}$ wafers, in which the silica layer is used as a dielectric, represent the easiest strategy to design a back gate FET from a film of $\mathrm{HgSS}^{194} \mathrm{HgSe},{ }^{29,317}$ and $\mathrm{HgTe} e^{35,36,314} \mathrm{NCs}$ due to their commercial availability. As a bias is applied across this layer, surface charges are generated. These charges are screened by the charges injected by the electrodes into the NCs. Dry oxide is beneficial to limit the breakdown and allows for a high gate bias operation. ${ }^{36,316}$ Silica gating can lead to large current modulations $\left(10^{7}\right.$ at $50 \mathrm{~K}$ has been reported $\left.{ }^{35}\right)$ but requires a high gate bias operation ( $50 \mathrm{~V}$ and more) due to its low dielectric constant (3.9). Consequently, insulators with a higher dielectric constant, such as alumina, have been used for the design of $\mathrm{HgX} \mathrm{NC}$-based FETs, ${ }^{314,315}$ reducing the gate bias range to below $10 \mathrm{~V}$ for the same current modulation. Using this approach, Yun et al. combined p-type HgTe NCs with n-type HgSe NCs to design NOR and NAND logic gates. ${ }^{329}$

Table 5 Figures of merit for field effect transistors based on $\mathrm{HgX}$ nanocrystal arrays

\begin{tabular}{|c|c|c|c|c|c|c|c|}
\hline $\mathrm{HgX}$ & $\begin{array}{c}\text { Surface } \\
\text { Chemistry }\end{array}$ & $\begin{array}{c}\text { On/Off } \\
\text { ratio }\end{array}$ & $\begin{array}{c}\text { Temperature } \\
\text { (K) }\end{array}$ & $\begin{array}{c}\mu_{\mathrm{e}} / \mu_{\mathrm{h}} \\
\left(\mathrm{cm}^{2} \cdot(\mathrm{V} \cdot \mathrm{s})^{-1}\right)\end{array}$ & $\begin{array}{l}\text { Gating } \\
\text { method }\end{array}$ & Comments & Year/Ref \\
\hline HgTe & $\begin{array}{c}\text { Sintering at } \\
150^{\circ} \mathrm{C}\end{array}$ & $10^{3}$ & RT & $-/ \approx 2$ & $\begin{array}{c}\mathrm{SiO}_{2} / \mathrm{Al}_{2} \mathrm{O} \\
3\end{array}$ & Dual gate & $2006 / 314$ \\
\hline HgTe & $\begin{array}{c}\text { Likely } \mathrm{OH}^{-} \\
+\mathrm{Al}_{2} \mathrm{O}_{3} \text { by } \\
\mathrm{ALD}\end{array}$ & $10^{3}$ & RT & $-/ 1$ & $\mathrm{Al}_{2} \mathrm{O}_{3}$ & Flexible & $2008 /{ }^{315}$ \\
\hline HgTe & $\begin{array}{c}\mathrm{MPOH} / \mathrm{HgC} \\
\mathrm{I}_{2}\end{array}$ & $10^{3}$ & 220 & $410^{-2} / 1.510^{-2}$ & $\mathrm{LaF}_{3}$ & Ionic glass & $2019 /{ }^{318}$ \\
\hline HgTe & $\begin{array}{c}\mathrm{MPOH} / \mathrm{HgC} \\
\mathrm{I}_{2}\end{array}$ & $10^{3}$ & 80 & $4.5 / 0.3$ & $\mathrm{SiO}_{2}$ & $\begin{array}{l}\text { High } \\
\text { mobility }\end{array}$ & $2019 /{ }^{36}$ \\
\hline $\mathrm{HgS}$ & $\mathrm{SCN}$ & 200 & RT & $1.5 / \mathrm{NR}$ & $\mathrm{SiO}_{2}$ & Annealed & $2017 / /^{194}$ \\
\hline HgTe & $\begin{array}{c}1, \text { Thioglyce } \\
\text { rol }\end{array}$ & 20 & RT & $\mathrm{NR} / 10^{-3}$ & $\mathrm{SiO}_{2}$ & $\begin{array}{c}\text { Spray } \\
\text { coating }\end{array}$ & $2017 / 330$ \\
\hline HgTe & $\begin{array}{c}1,7 \\
\text { heptanedit } \\
\text { hiol }\end{array}$ & 200 & RT & $0.1 / 0.1$ & $\begin{array}{l}\text { Electroch } \\
\text { emical }\end{array}$ & & $2012 / 266$ \\
\hline HgTe & $\begin{array}{c}\mathrm{MPOH} / \mathrm{HgC} \\
\mathrm{I}_{2}\end{array}$ & $310^{3}$ & 75 & $8 / 1.5$ & $\mathrm{SiO}_{2}$ & $\begin{array}{c}\text { High } \\
\text { mobility }\end{array}$ & $2020 / 316$ \\
\hline HgTe & $\mathrm{As}_{2} \mathrm{~S}_{3}$ & $\begin{array}{l}<10 \\
@ R T\end{array}$ & & $310^{-2} / 110^{-2}$ & $\mathrm{SiO}_{2}$ & & $2013 /{ }^{35}$ \\
\hline
\end{tabular}


$10^{7}$ at

$45 \mathrm{~K}$

\begin{tabular}{cccccccc}
\hline HgSe & $\mathrm{As}_{2} \mathrm{~S}_{3}$ & 100 & $\mathrm{RT}$ & $90 / \mathrm{NR}$ & $\begin{array}{c}\mathrm{SiO}_{2} / \text { elect } \\
\text { rolyte }\end{array}$ & Intraband & $2016 /{ }^{29}$ \\
\hline HgSe & $\begin{array}{c}\mathrm{MPOH} / \mathrm{HgC} \\
\mathrm{I}_{2}\end{array}$ & 100 & 80 & $0.7 / 0.3$ & $\mathrm{SiO}_{2}$ & $2020 /{ }^{317}$ \\
\hline \hline
\end{tabular}

Alternatives to dielectrics have also been explored to realize the gating of $\mathrm{HgX} \mathrm{NC}$ arrays. The quest for such alternatives was first motivated by the design of higher capacitance gates to control the carrier density in self-doped NCs. Electrolytes can be a strategy to induce gate effect in wide bandgap NCs, such as $\mathrm{CdSe}^{331,332}$ or ZnO. ${ }^{333}$ Electrolyte gating of $\mathrm{HgTe}^{30,96,262,266,278}$ and $\mathrm{HgSe}^{29,120,122}$ NCs has also been well developed. An electrolyte is a dispersion of ions within a liquid or a polymer matrix; ${ }^{158}$ see Figure $31 \mathrm{c}$. As a gate bias is applied, the ions can percolate within the NC array, and the electrodes inject carriers to screen these charges. Moreover, intercalation of the ions within the NC atomic lattice has been reported. ${ }^{334}$ In the electrolyte, gate capacitance is driven by the ratio of the dielectric constants on each side of the ionic double layer formed at the interface of the NCs. This double layer is extremely thin $(1 \mathrm{~nm})$, and the gate capacitance is consequently much larger $\left(\approx 1 \mu \mathrm{F} . \mathrm{cm}^{-2}\right)$ than that for dielectric materials $(\approx 10$ $\mathrm{nF} . \mathrm{cm}^{-2}$ for $\left.400 \mathrm{~nm} \mathrm{SiO}\right)_{2}$ ). Because of the percolation of ions within the NC array, it is possible to obtain a gate effect in NC films, which is of key interest to design phototransistors in which both large absorption and gate tunability are required. The large gate capacitance enables the realization of a low bias operation ( $<3 \mathrm{~V}$, as shown in Figure 31d). Nevertheless, the gate effect is based on the displacement of ions; this process is intrinsically slow and thus limits the sweep rate (Figure 31f) and requires an operation of the device above the freezing point of the matrix (Figure 31e). The last point allows the preparation of a device in a given charging configuration at room temperature before cooling it below the electrolyte freezing point; however, this approach cannot be used to realize the low temperature tuning of the carrier density.
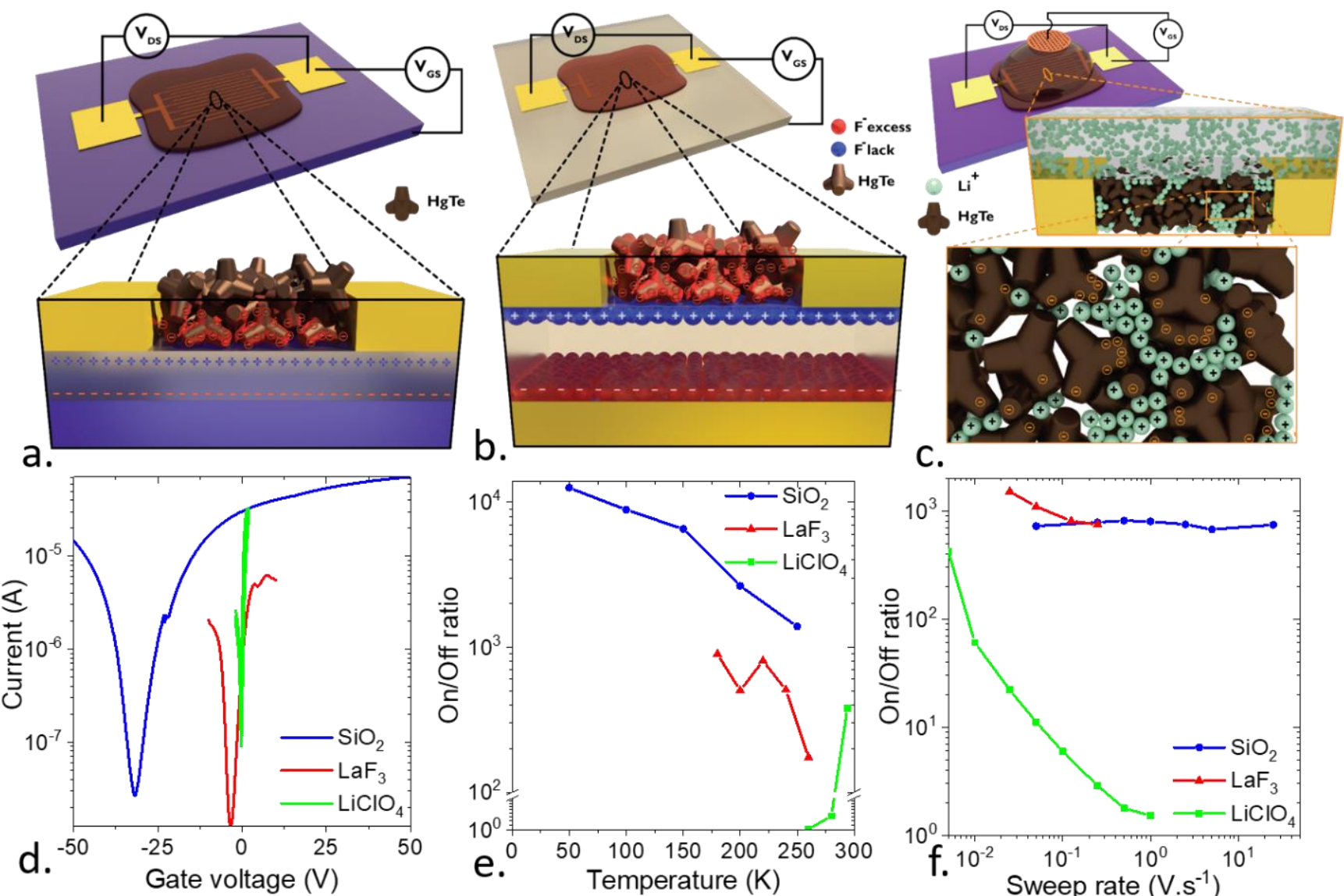

C.
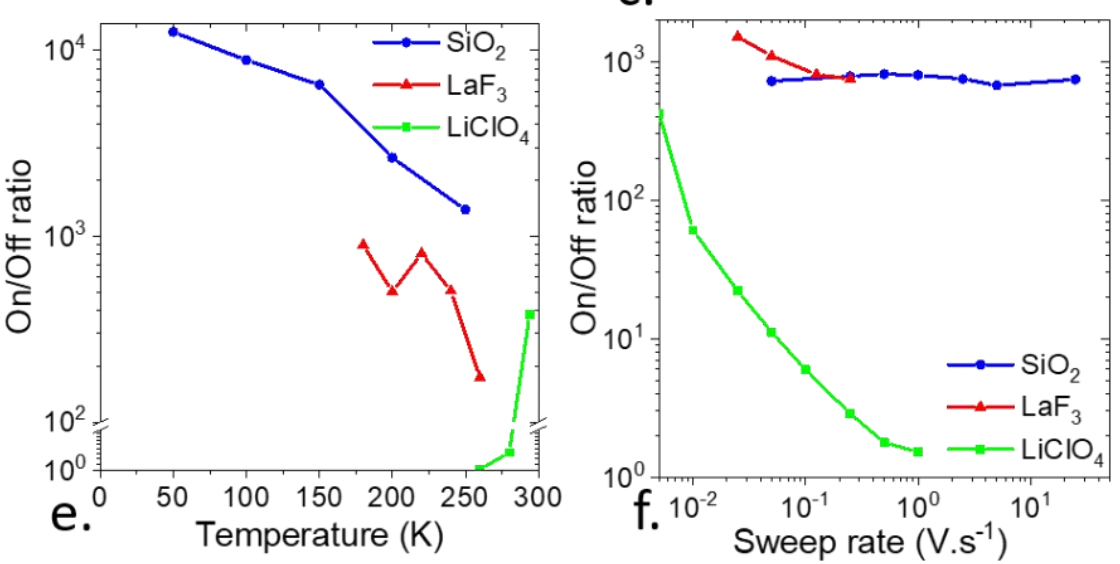

Figure 31 Schemes of HgTe back gate FETs on (a) $\mathrm{SiO}_{2}$ and (b) $\mathrm{LaF}_{3}$ when $\mathrm{V}_{g s}>0$. c. Scheme of a $\mathrm{HgTe}$ top gate FET with an electrolyte ( $\mathrm{LiClO}_{4}$ in a PEG matrix). d. Typical transfer curves obtained for $\mathrm{HgTe}$ nanocrystals with different gating techniques. $\mathrm{SiO}_{2}$ and $\mathrm{LaF}_{3}$ are measured at $200 \mathrm{~K}$, and the electrolyte is measured at $300 \mathrm{~K}$. e. On/off ratios of FET curves measured at different temperatures for different gating configurations. The ratios are normalized according to the ratio at the highest temperature for each gating configuration. $f$. On/off ratios normalized according to the ratio at the lowest sweep rate for each gating configuration measured at different sweep rates of $V_{G S}$ for each 
To overcome this difficulty, Gréboval et al. proposed the use of ionic glass ${ }^{335}$ to gate HgTe NC films. ${ }^{108,318}$ The authors used a LaF $_{3}$ substrate, which is also an IR transparent material. Such glass types include fluorine vacancies ( $F^{-}$vacancies) that can move to the surface to induce polarization under an applied bias; ${ }^{336}$ see Figure $31 \mathrm{~b}$. Ionic glass also has a large capacitance, allowing low bias operation (see Figure 31d). Vacancy displacement is more efficient than ion diffusion in the electrolyte matrix, which enables a higher sweep rate and operation down to $150 \mathrm{~K}$. The latter temperature of the electrolyte is determined by the vacancy freezing point (Figure 31f). In this sense, ionic glass gating combines the advantages of electrolytes with solid state handling, ${ }^{337,338}$ which releases the constraint from manipulating liquids/polymers.

More recently, the same group explored a complementary approach for the gating of HgX NC films. High capacitance methods (electrolytes or ionic glasses) rely on ion displacement, which corresponds to two intrinsic limitations: (i) slow operation limiting the sweep rate and (ii) a relatively high operating temperature (above the freezing point, which is always above $200 \mathrm{~K}$ ). The latter point is detrimental for the design of phototransistors dedicated to IR detection since cryogenic conditions are required, especially for the detection of long IR wavelengths. For example, the operating temperature of a $5 \mu \mathrm{m}$ cutoff wavelength camera based on epitaxially grown semiconductors remains below $150 \mathrm{~K}$, dropping by approximately $90 \mathrm{~K}$ for $10 \mu \mathrm{m}$ detection and as low as $4 \mathrm{~K}$ in the $\mathrm{THz}$ range. Consequently, a gating method to accommodate low temperature operations while still offering a large capacitance and possibly without the limitation of $\mathrm{SiO}_{2}$ (breakdown) is of considerable interest. Gréboval et al. explored a ferroelectric gating strategy. ${ }^{339}$ At the Curie temperature of a ferroelectric crystal $\left(T_{c}\right)$, the material transitions from a paraelectric to a ferroelectric phase, which induces a divergence in the dielectric constant, as depicted in Figure 32a. To obtain low temperature gating, the authors used $\mathrm{SrTiO}_{3}$, a frustrated ferroelectric (i.e., with a negative $\mathrm{T}_{c}$ ), also known as a quantum paraelectric. The dielectric constant strongly rises below $100 \mathrm{~K}$ and can reach 30000 below $40 \mathrm{~K}$. At this temperature, the dielectric constant saturates owing to quantum fluctuation. ${ }^{340}$ Such gating has been explored previously for a $2 \mathrm{D}$ superconducting electron gas obtained at the $\mathrm{LaAlO}_{3} / \mathrm{SrTiO}_{3}$ interface. ${ }^{341,342}$ Gréboval et al. applied this gating to $\mathrm{HgTe} \mathrm{NCs}$ with various band gaps from 720 to $250 \mathrm{meV}$ and obtained a large current modulation $\left(>10^{3}\right)^{339}$ owing to the use of the $\mathrm{SrTiO}_{3}$ gate. The reported capacitance is equivalent to that of an $80 \mathrm{~nm}$ thick $\mathrm{SiO}_{2}$ film. Compared with thin silica films, quantum paraelectric gating is a breakdown free/low leakage gating method. A bias of as large as $200 \mathrm{~V}$ can be applied without any risk of device destruction (i.e., without electrical breakdown of the gate). This method is especially promising owing to the recent progress in thin film deposition of oxide and ferroelectric materials.
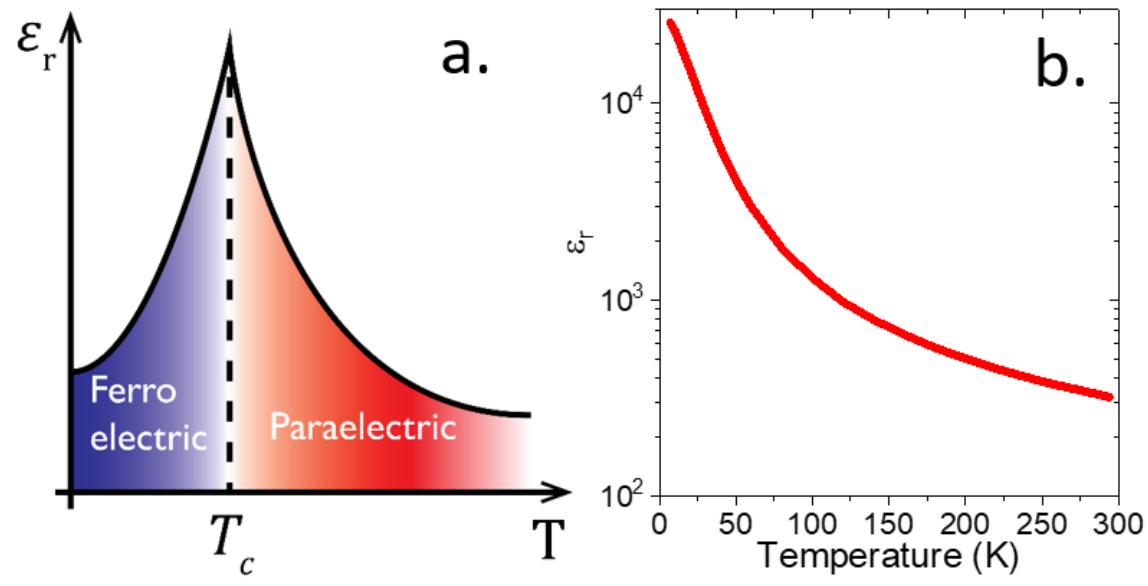

Figure 32 a. Scheme of the divergence of the dielectric constant around the Curie temperature as a paraelectric switch toward its ferroelectric phase. b. Dielectric constant dependence on the temperature of the STO substrate used as the gate. Parts $b$ is adapted with permission from ref ${ }^{339}$ Copyright (2021), American Chemical Society.

While it is unlikely that $\mathrm{HgX} \mathrm{NC-based} \mathrm{FETs} \mathrm{can} \mathrm{compete} \mathrm{with} \mathrm{Si-based} \mathrm{technologies} \mathrm{for} \mathrm{electronics,} \mathrm{FET} \mathrm{appears} \mathrm{to} \mathrm{be} \mathrm{a} \mathrm{promising}$ spectroscopic tool to investigate the confined forms of $\mathrm{HgX}$. In addition to the determination of the majority carriers and internanoparticle coupling through mobility, observation of a Pauli blockade can be used to determine and finely tune the state filling of NC quantum states. The concept was initially introduced for CdSe NCs, while Guyot-Sionnest and coworkers observed nonmonotonic mobility behavior as the $1 \mathrm{~S}_{\mathrm{e}}$ state is filled using electrolytic gating. ${ }^{343}$ The $1 \mathrm{~S}_{\mathrm{e}}$ state presents a twofold degeneracy; thus, when the Fermi level is brought in resonance with the $1 \mathrm{~S}_{\mathrm{e}}$ state, the mobility is maximized for a state filling of 1 carrier per 
NC. For higher fillings, the film conductance drops until the $1 \mathrm{P}_{\mathrm{e}}$ state starts to be filled. This drop in mobility is a result of the fact that in the regime between 1 and 2 carriers per NC on average, certain NCs exhibit a fully filled ( 2 carriers) 1Se state. Consequently, these NCs cannot contribute to transport, as the only way for carriers to move is to pass through the $1 \mathrm{P}_{\mathrm{e}}$ state, which requires overcoming the $1 \mathrm{P}_{\mathrm{e}}-1 \mathrm{~S}_{\mathrm{e}}$ energy barrier. Using a dual gate configuration (bottom $\mathrm{SiO}_{2}$ gate + top electrolytic gate), Lhuillier et al. demonstrated that a similar behavior can be observed in an array of monodisperse HgSe NCs, ${ }^{29}$ as shown in Figure 33 a. This observation was later confirmed by Chen et al. by using only a bottom dielectric gate, discussed as state resolved transport. ${ }^{317}$ The observation of a Pauli blockade in HgTe occurred only later. This delay is related to the fact that the observation of a Pauli blockade requires more monodisperse syntheses, which, until recently, was not the case for HgTe NCs. The introduction of new synthetic procedures by Shen et al. ${ }^{63}$ enabled the growth of spherical and monodisperse HgTe, leading to the observation of these stateresolved transfer curves. ${ }^{316}$

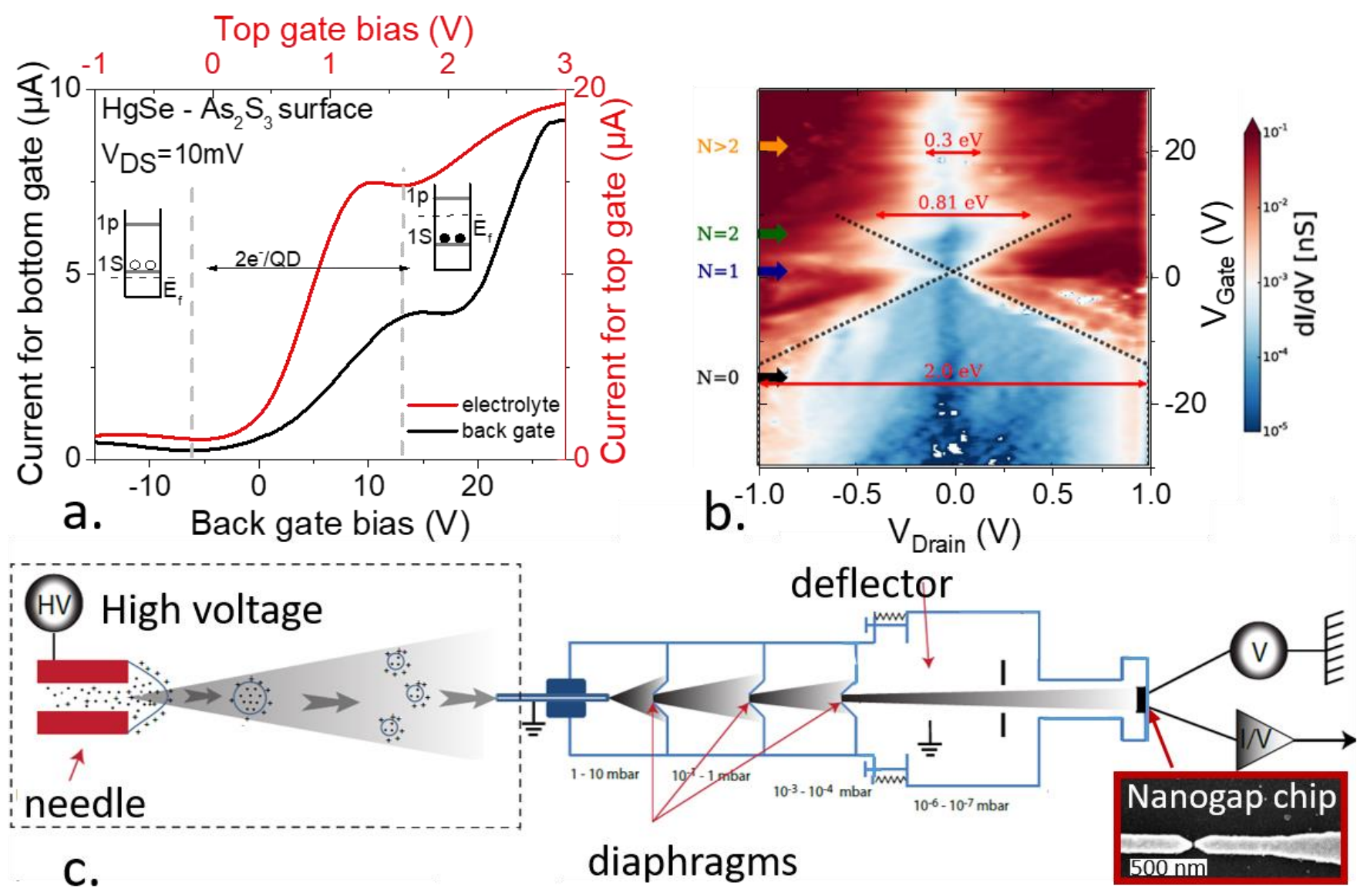

Figure 33 a. Transfer curves (drain current vs. gate voltage) for a thin HgSe CQD film using back and electrolytic gating. In both cases, the drain source bias is $10 \mathrm{mV}$. Part a is adapted with permission from ref ${ }^{29}$ Copyright (2016), American Chemical Society. b. Log scale color map of the differential conductance $\mathrm{dl} / \mathrm{dV}$ as a function of the drain and gate voltages for a HgSe single NC. The black dashed lines are a visual guide for the closing of the gap as the 1 Se level crosses the Fermi level energy. $C$. Sketch of the electrospray setup utilized to fabricate the single QD junctions. Parts $b$ and $c$ are adapted with permission from ref ${ }^{259}$ Copyright (2017), American Chemical Society.

Aubin and coworkers further investigated state-resolved transport in $\mathrm{HgSe} \mathrm{NCs}^{259}$ by conducting single particle measurements. The strategy for a single NC-based experiment usually relies on the measurement of a single NC PL signal. However, single particle measurements become especially difficult when using infrared materials due to their low PL efficiency and the lack of efficient single photodetectors in the infrared region. The strategy proposed by Aubin and coworkers is based on chip tunnel spectroscopy. Compared to conventional scanning tunneling spectroscopy, this approach offers higher temporal stability and enables gating. The abovementioned method is based on two electrodes spaced by 5-10 $\mathrm{nm}$ (i.e., nanogap), prepared by e-beam lithography. A solution of nanoparticles is sprayed under vacuum on a chip including 32 nanogaps; see the scheme of the setup in Figure $33 \mathrm{c}$. After each spray, the conductance through the nanogap electrodes is measured, and a sudden increase in conductance indicates particle trapping. The method has been applied to a variety of nanoparticles, including gold, ${ }^{344} \mathrm{Fe}_{3} \mathrm{O}_{4},{ }^{345} \mathrm{PbS}^{346}$ and $\mathrm{HgSe}^{259} \mathrm{NCs}^{3}$ Once the particle is trapped, on-chip electron tunnel spectroscopy can be conducted at a low temperature. A typical tunnel 
spectroscopy map is shown in Figure 33b. As a function of the filling with the gate, two bandgaps are observed. The large gap, under hole injection (i.e., negative gate bias), corresponds to the interband gap, while the smaller gap, under electron injection, corresponds to the intraband gap. The obtained values can be fully correlated with optical measurements from IR spectroscopy. This result indicates that self doping is present at the single particle level and that intraband absorption is not the consequence of collective behavior. Finally, the authors also reported on photoconduction from a single HgSe NC. 


\section{PHOTOCONDUCTION AND IR DETECTION}

The last section of this review is dedicated to infrared sensing based on $\mathrm{HgX} \mathrm{NCs}$. Bulk HgCdTe alloy is the most mature material for infrared detection because of its high performance and wide tunability from the near-infrared region to the long-wave infrared region. In this material, spectral tunability is obtained by alloying and controlling the Cd fraction. In HgTe NCs, the same magnitude of tunability is enabled through confinement control, which renders HgTe NCs a natural replacement for HgCdTe in the future design of IR sensors. However, IR detection applications only appeared 10 years after the introduction of mercury chalcogenide NCs, whose first application was focused on light emission. This low interest in HgX NC-based IR detection in the early 2000 s was likely due to the inferior transport properties of the NCs available at that time. During the 2000-2010 period, substantial progress in ligand exchange procedures was made, allowing the design of high mobility films. This aspect demonstrated that NCs could be used for IR detector design, even though the approaches were not immediately applied to films of HgX NCs.

This chapter is organized as follows. We first redefine the main figures of merit relative to infrared detection, including noise, since this quantity is often wrongly evaluated. In this discussion, we also mention the experimental setups used to measure these figures of merit. Next, we discuss different strategies for the devices that have been developed. First, we consider photoconductors and later discuss the refinements of the planar geometry, including phototransistors, flexible devices, intraband detection, and coupling to 2D materials. The following section is focused on photodiodes, mostly relying on vertical geometry devices. This section is valuable since all of the highest performing devices are based on this geometry. The final sections of the chapter are dedicated to three key recent developments that enhance the performance and maturity of IR detection based on NCS. These developments include (i) multicolor sensing, (ii) detectors with enhanced light-matter coupling and (iii) multipixel devices and focal plane arrays.

\subsection{Figures of merit and their experimental determination}

Figures of merit are used to compare the performance of different types of photodetectors (e.g., different working principles, channel materials, and geometries). In the following discussion, we briefly introduce the general figures of merit and their experimental measurements.

\subsubsection{Spectral response}

The term "infrared" describes a broad part of the optical spectrum at longer wavelengths than those of the visible ranges. The infrared region is commonly subdivided into three main domains: the near-infrared region (1-3 $\mu \mathrm{m})$, midinfrared region (3-20 $\mu \mathrm{m})$, which corresponds to the range of blackbody emission close to room temperature and in which most chemical bonds present resonances, and far-infrared region, which strongly overlaps with the THz range. In regard to IR sensing and imaging, the subdivisions are even more defined, especially to account for the transparency ranges of the atmosphere and the region in which it is possible to conduct long distance imaging. The following definitions can be considered:

Near infrared (NIR): NIR is close to the visible spectrum and spans from $700 \mathrm{~nm}$ to $900 \mathrm{~nm}$. This range overlaps with the absorption of silicon. Target applications in the NIR range are divided into biological imaging and fluorescence detection.

Shortwave Infrared (SWIR): SWIR is defined as the wavelength range from $1 \mu \mathrm{m}$ to $1.7 \mu \mathrm{m}$. The latter value is arbitrary since it corresponds to the bandgap of InGaAs detectors, which are the most mature detectors in this range of wavelengths. In this range, several applications are possible, including active imaging, night glow-assisted imaging, and tissue imaging. It is also common to define SWIR in the range between $1.7 \mu \mathrm{m}$ and $2.5 \mu \mathrm{m}$. In SWIR, the contrast in an image is, as in the visible region, the result of the scattered light from an external source.

Midwave Infrared (MWIR): MWIR extends from 3 to $5 \mu \mathrm{m}$ and corresponds to an atmospheric transparency window. Above $3 \mu \mathrm{m}$, blackbody emission starts to prevail over reflection from external sources, enabling thermal imaging. In MWIR, hot objects are typically targeted. Moreover, thermal imaging can be conducted at room temperature with a greater contrast than in LWIR, albeit at the cost of a smaller number of photons. In this range, narrow bandgap semiconductors, such as InSb and $\mathrm{HgCdTe}$ and other type II superlattices based on InAs/GaSb, are conventional materials used for photodetection and in applications such as thermal imaging and gas sensing.

Longwave infrared (LWIR): LWIR ranges from 8 to $12 \mu \mathrm{m}$. This range is well matched to the maximum emission of a blackbody at room temperature $(10 \mu \mathrm{m})$. This range is thus a useful range to perform passive thermal imaging. Ternary alloy materials, such as $\mathrm{HgCdTe}$ and type II superlattices, are the traditional materials used for LWIR detection. This range is also the operating range of bolometers, energy detectors used in most mass market thermal detectors and cameras. Detectors in LWIR are applied in industrial inspection, defense, and astronomy. 
Terahertz (THz): Terahertz ranges from 30 to $200 \mu \mathrm{m}$. Bolometers that operate at low temperature are typically used for THz detection. This range of wavelengths is used in astronomy and to conduct nonionizing surveillance (mostly for safety applications). In the $\mathrm{THz}$ range, various materials that are opaque in the visible region become transparent.

The classical techniques for detector characterization typically employ a monochromator, which is an integrating sphere, and rely on measuring the signal on a calibrated detector. In the infrared range, such techniques are difficult to set up. One convenient way to measure the spectral response is to perform Fourier transform infrared spectroscopy (FTIR) in the continuous scan mode. The spectral response is measured by placing the detector in a FTIR instrument and amplifying the current with a low noise amplifier before sending the signal back to the FTIR electronics to perform a Fourier transform. In this case, the collected signal typically comes from the spectrum of the source multiplied by the transmission of the optics and by the spectral response of the detector. The latter is the signal of interest. A reference is thus required to extract the spectral response of the detector from this product. This background is generally attained by using the signal collected through the internal DTGS detector of the FTIR, which has a flat spectral response. Consequently, the signal measured on the DTGS is simply the product of the source spectrum by the transmission of the optics. Dividing the signal acquired on the unknown detector by that from the DTGS leads to the spectral response of the detector.

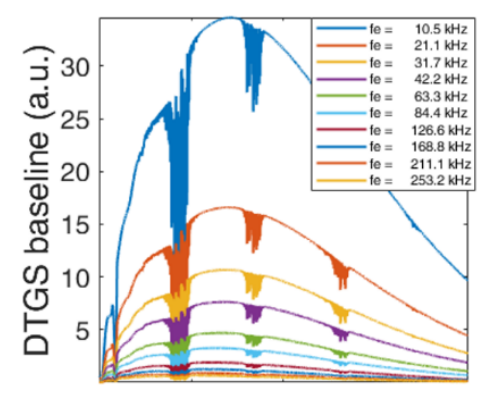

a. $2000400060008000 \quad 10000$ Wavenumber $\left(\mathrm{cm}^{-1}\right)$

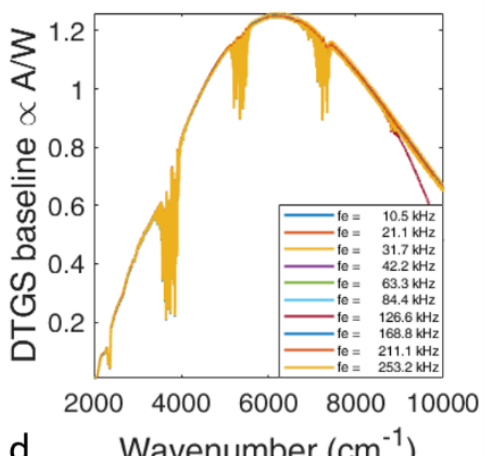

d. Wavenumber $\left(\mathrm{cm}^{-1}\right)$
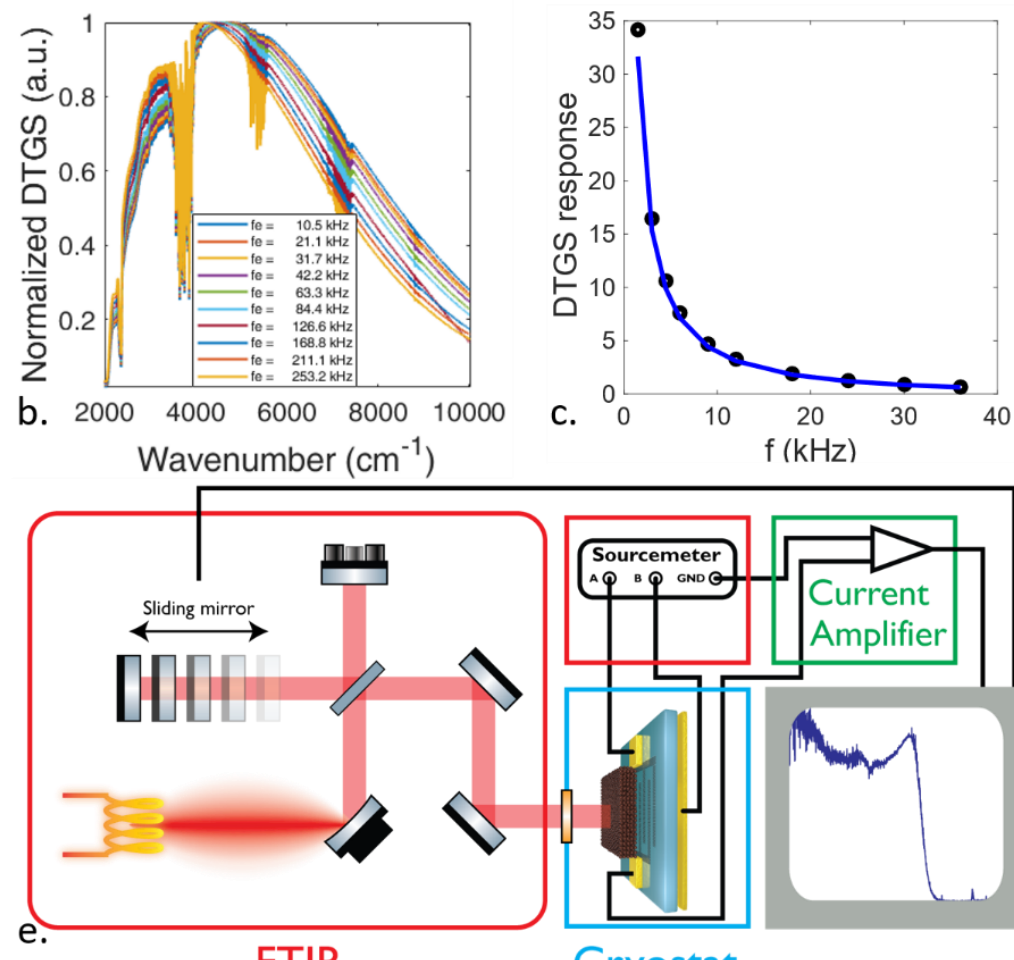

FTIR

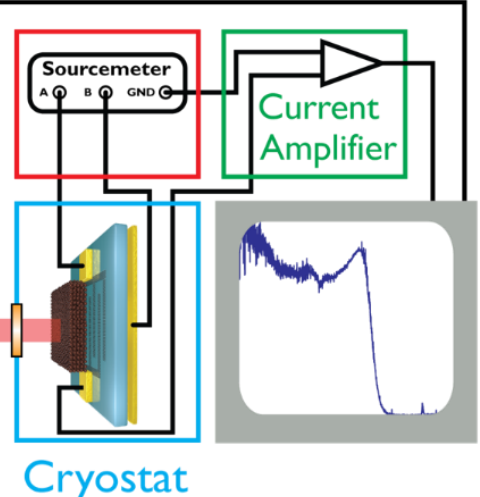

Cryostat

Figure 34 a. DTGS signal of an FTIR source measured at different sampling frequencies. b. Normalized DTGS signal at different sampling frequencies. c. Estimated transfer function at $4500 \mathrm{~cm}^{-1}$ and its fit by $H(f)=\left(a+b f+c f^{2}+d f^{3}\right)^{-1}$. $d$. DTGS source spectra at different sampling frequencies corrected by the transfer function. e. Scheme of a typical setup used to measure the spectral response. FTIR illuminates the sample that is biased by a source meter and can eventually be placed in a cryostat. The current is later amplified by a transimpedance amplifier and sent back to the FTIR electronics to compute the Fourier transform.

However, as explained by Gravrand et al., ${ }^{347}$ this spectrum should be corrected by the transfer function of the instrument, taking into account the low response time of the DTGS detector; see Figure 34a and b. The measured signal (after normalization by the DTGS background) is the result of the product of this transfer function by the detector's real spectral response. The transfer function can be estimated by measuring the IR source spectra at different sampling frequencies with the DTGS detector. The transfer function can be estimated as $H(f)=\frac{1}{a+b f+c f^{2}+d f^{3}}$, where $f$ is the temporal frequency associated with the wavenumber after the Fourier transform; $=R R \times f_{e} \times \sigma$, where RR is the retardation rate of the laser, $f_{e}$ is the sampling frequency and $\sigma$ is the wavenumber; see Figure 34c. Once the fitting parameters $a, b, c$, and $d$ are identified, the source spectrum is divided by the transfer function to ensure that the response of the detector becomes independent of the acquisition speed (see Figure 34d). The 
obtained spectrum is at this stage is specified in relative units and must be calibrated in $A$. $W^{-1}$, which requires precise knowledge of the incident photon flux. Another way of rescaling the spectral response in $A . W^{-1}$ is to measure the exact device responsivity at a given wavelength with a known incident flux (e.g., with a laser diode).

\subsubsection{Operation temperature range}

To reduce the dark current in the device and its associated noise, low temperature operation is required to achieve high signalto-noise ratios in the infrared domain. A larger targeted wavelength corresponds to a lower operating temperature. Cryogenic temperature operation is associated with a high cost, weight, and constraints regarding the lifetime. Consequently, most research on IR detection is focused on increasing the operating temperature. In the SWIR, room temperature operation is possible, but it is common to use a Peltier stage, which keeps the operating temperature as constant as possible. Depending on the targeted performance, cooling can also be implemented in SWIR, generally over a range of temperatures that is compatible with the Peltier stage. In MWIR, cryogenic operation is usually performed. In the blue part of MWIR (i.e., below the $\mathrm{CO}_{2}$ absorption at $4.2 \mu \mathrm{m}$ ), state-of-the-art operating conditions can attain temperatures of up to $150 \mathrm{~K}$, while for the red part of MWIR, the usual operating temperature is approximately $120 \mathrm{~K}$. In LWIR, operating conditions immediately above the temperature of liquid nitrogen can be achieved. In THz, liquid He operating conditions are usually employed. These constraints must be considered to highlight the level of performance required for the NC-based IR sensors. In particular, in SWIR, low cost sensing requires room temperature operation.

\subsubsection{Responsivity $\left(R, A . W^{-1}\right)$}

Responsivity $(R)$ is the ability of a material to convert incident light into an electrical signal and is a common indicator to evaluate the performance of photoconductive devices. Responsivity is the ratio of the detector's photocurrent ( $\left.I_{\mathrm{ph}}\right)$ to the total incident optical power $\left(P_{\text {in }}\right)$ that it receives. This value can be simply described as $R=I_{\mathrm{ph}} / P_{\text {in }}$.

In nanocrystal devices, $R$ is strongly dependent on the incident flux. In general, a higher photon flux corresponds to a weaker response. Because of this behavior, the $R$ values reported using a blackbody (low photon flux source) are generally higher than those measured using laser sources. Moreover, it is important to investigate the frequency dependence of $R$ to determine the range of operating frequencies of the device.

Responsivity is usually measured at a given frequency, indicating that the sample is illuminated by electrically or mechanically chopped light. This light can either be a laser or a filtered blackbody. In the case of laser illumination, the power of the laser is directly measured using a calibrated detector. For blackbody radiation, the power can be calculated using the following formula:

$$
P(W)=A_{d} \pi \cdot \cos (\theta) \cdot \sin ^{2}(\alpha) \int_{\lambda_{\min }}^{\lambda_{\max }} \frac{2 h c^{2}}{\lambda^{5}} \frac{1}{e^{\frac{h c}{\lambda k T}}-1} d \lambda
$$

where $A_{d}$ is the area of the device, $\theta$ is the incidence angle, $\alpha$ is the half angle of the view under which the blackbody is detected by the device, $\lambda_{\min }$ and $\lambda_{\max }$ are the border wavelengths of the range of interest (taken as the cutoff wavelength of the filter and measured detector, respectively), $h$ is Planck's constant, $c$ is the speed of light, $k$ is the Boltzmann constant and $T$ is the blackbody temperature.

Once the power is determined, the photocurrent can be measured either with an oscilloscope or, if the chopping signal is a sine curve, with a lock in amplifier, as is shown in Figure 35a and b. The ratio between the photocurrent and incident power yields the responsivity in $A^{-1} W^{-1}$. 

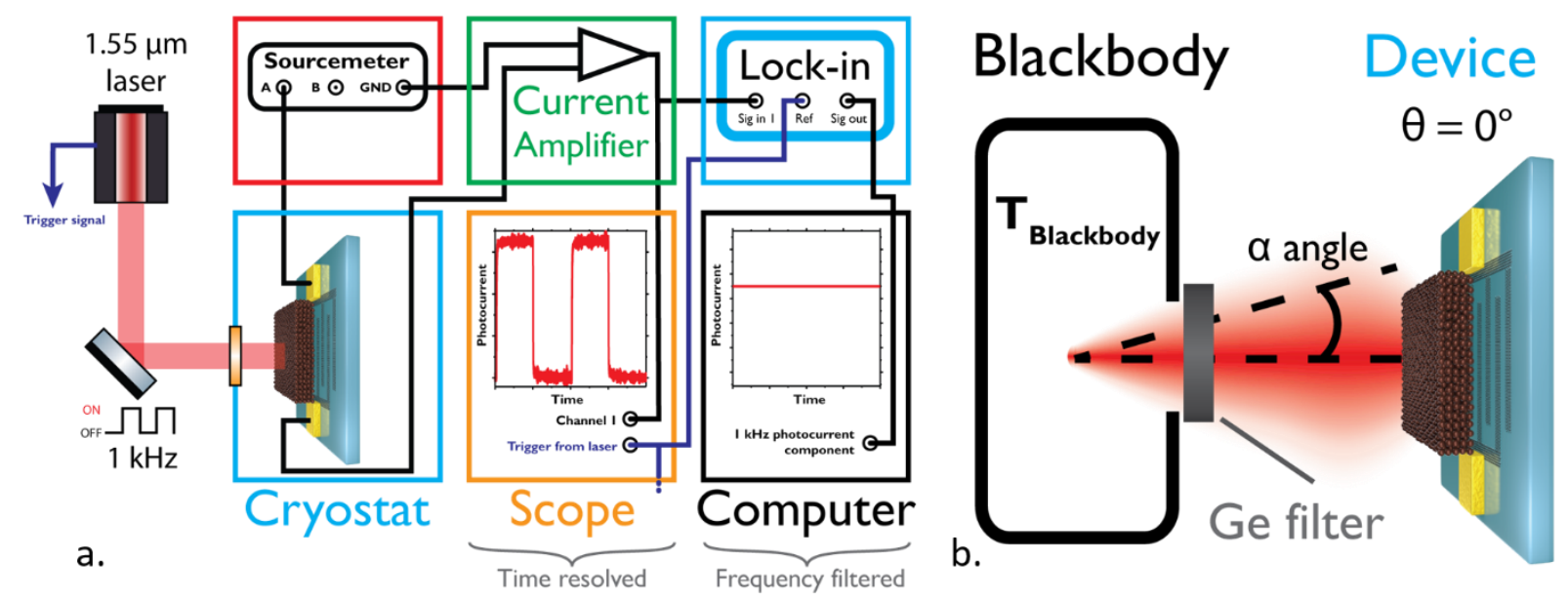

Figure 35 a. Scheme of an experimental setup to measure the photocurrent with an electrically chopped laser at $1.55 \mu \mathrm{m}$. The signal is biased with a sourcemeter, and the current is amplified through a transimpedance amplifier (TIA). The photocurrent can either be measured by a scope in a timeresolved setup or, for low SNR samples, a lock-in amplifier can be used to filter all the other frequencies of the signal. $b$. Scheme of a response setup with blackbody illumination. The device is at a normal incidence $\left(\theta=0^{\circ}\right)$, $\alpha$ is the half viewing angle of the device by the blackbody, $T_{\text {Blackbody }}$ is the temperature of the blackbody and the germanium filter is a high pass filter with a $1.8 \mu \mathrm{m}$ cutoff.

\subsubsection{Current noise spectral density $\left(\delta / \mathrm{n}\right.$ in $\mathrm{A}$. $\left.\mathrm{Hz}^{-1 / 2}\right)$}

The current noise spectral density is highly specific to the infrared region. Because of the narrow bandgaps of the materials used for IR detection, optical excitation is always in competition with thermal excitation. Consequently, the signal-to-noise ratio is weak compared to that for visible and UV detectors. The noise in the photodetector mainly consists of (i) Johnson noise, (ii) shot noise, and (iii) flicker noise $(1 / f)$. (i) The thermal noise $\delta /$ th is related to the fluctuation of the carrier density in any object, which is a common behavior that is not specific to conductive materials. The corresponding value can be easily estimated as $\delta \mathrm{thn}^{2}=4 \mathrm{kT} \Delta \mathrm{f} / \mathrm{Res}$, where $\mathrm{kT}$ is the thermal energy, $\Delta f$ is the frequency bandwidth of the measurement, and Res is the device resistance. This noise is white noise (i.e., independent relationship with frequency). (ii) The shot noise relates to the charge fluctuation resulting from the current flow in the device and can be defined as $\delta I_{G R}{ }^{2}=2$ egl, where e is the elementary charge, $I$ is the current flowing in the structure and $g$ is the photoconduction gain. As a form of thermal noise, this noise is white noise.

In nanocrystals, these two white noise sources rarely dominate. The main contribution originates from (iii) $1 / \mathrm{f}$ noise; see Figure $36 \mathrm{~b} .{ }^{348,349}$ Contrary to the previous sources of noise, there is no analytical expression to evaluate $1 / \mathrm{f}$ noise from easily measurable parameters $(R, I)$. Hooge's law provides an empirical expression ${ }^{350}$ for $1 / f$ noise $\delta I_{1 / f}^{2}=\frac{\alpha I^{2}}{\mathrm{Nf}}$, where $N$ is the total number of carriers involved in the transport, $l$ is the current, $f$ is the frequency and $\alpha$ is Hooge's constant over a large range of carrier concentrations. However, for arrays of NCs, Hooge's constant does not appear to be consistent ${ }^{279,348}$ and is larger ${ }^{279}$ (approximately 1) than the value reported by Hooge in metallic thin films $\left(\alpha<10^{-3}\right)$. In NC films, $\alpha$ appears to be independent of the material nature (such as metal, wide bandgap semiconductor or narrow bandgap semiconductor) and particle size. ${ }^{348}$ However, the quality of the film and, particularly, the internanoparticle coupling dramatically drive the actual magnitude of $\alpha$. A larger coupling corresponds to a smaller $\alpha$. Overall, in NC-based detectors, frequency-resolved measurements must be performed to determine the actual value of noise as it cannot be measured analytically. The usual assumption of using white noise as the limiting noise systematically underestimates noise, often by several orders of magnitude, which dramatically overestimates the signal-to-noise ratio (and the detectivity) of the device.

In practice, noise is usually measured by biasing the device with a battery. The current is amplified through a low noise amplifier and sent to a spectrum analyzer; see the scheme of the experimental setup in Figure 36a. The typically targeted range of frequency is $1 / t_{i}$, where $t_{i}$ is the targeted integration time. Generally, noise is acquired between $1 \mathrm{~Hz}$ and a few $\mathrm{kHz}$. A current amplifier whose bandwidth matches the experiment must be used, especially for highly resistive samples that require large gains. The reported noise needs to be sample limited and not amplifier limited. Additionally, for uncooled amplifiers, the lowest measurable noise is of the order of a few $\mathrm{fAHz}^{-1 / 2}$. This aspect can be a general limitation to measure noise in resistive samples (especially for wide bandgap samples). A key parameter for this measurement is the noise level of the room in which the measurement is performed. The sample and electronics must be isolated from other noise sources in the room. The first source of noise to be suppressed is the $50 \mathrm{~Hz}$ (or $60 \mathrm{~Hz}$, depending on the location) noise from the AC line. For highly conductive samples (low 
resistance), noise measurement may be more difficult to perform, and a Wheatstone bridge can be used to suppress the DC component of the signal, ${ }^{348}$ allowing the use of a higher amplification gain and resolution of the sample noise spectral density.
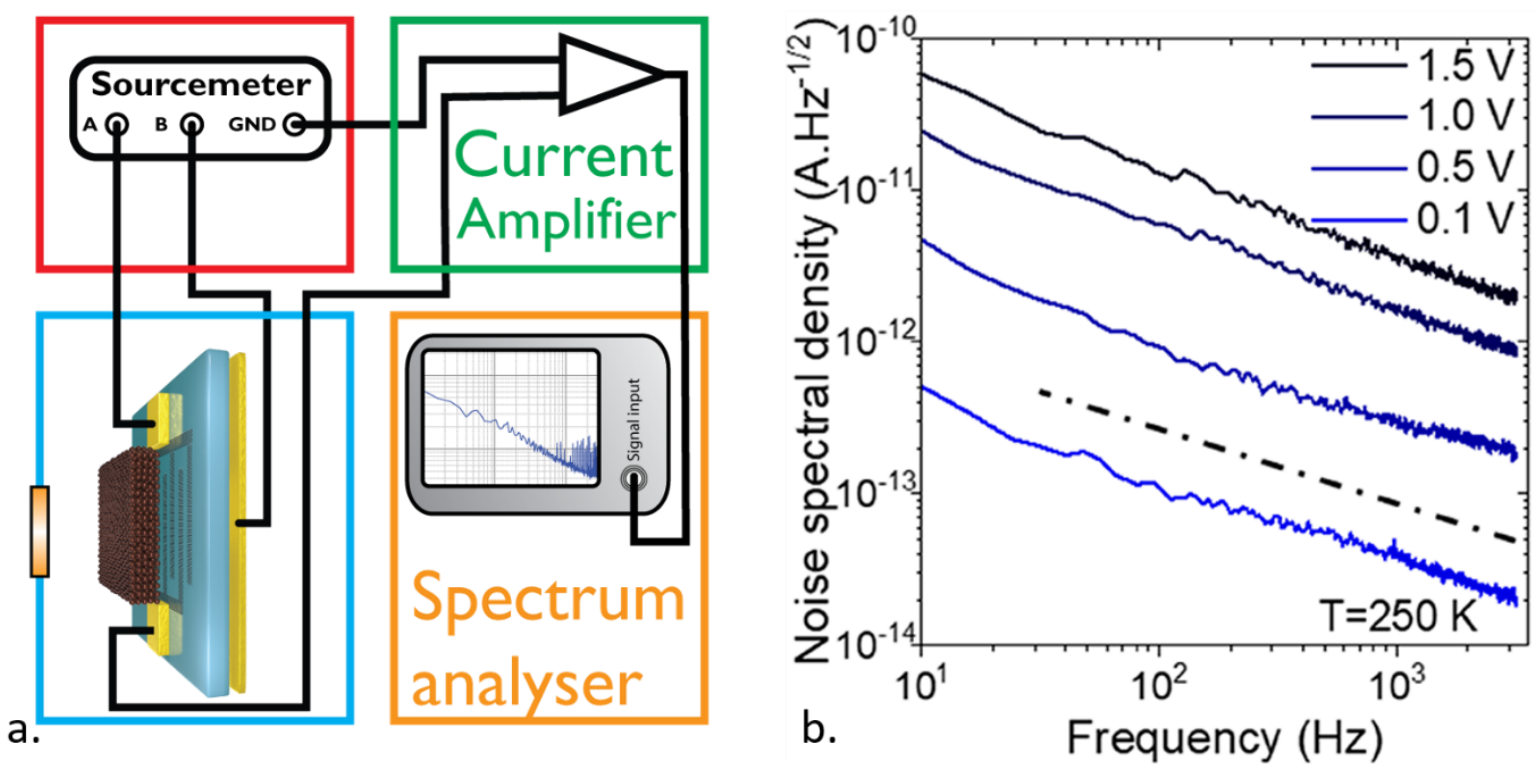

Figure 36 a. Scheme of a typical experimental setup for making a noise spectral density measurement. The sample is biased by a source meter (or a battery), and the current is amplified through a low noise transimpedance amplifier. The amplified signal is sent to a spectrum analyzer. $b$. Typical noise spectral density curves for a HgTe film under various biases at $250 \mathrm{~K}$. The dashed line represents the 1/f slope.

\subsubsection{Noise equivalent power (NEP, W/Hz $\mathrm{z}^{1 / 2}$ )}

NEP is the minimum optical power for a detector to output a signal-to-noise ratio of 1 with a $1 \mathrm{~Hz}$ bandwidth. This value can be expressed as NEP $=\delta / n / R$, where $\delta / n$ is the current noise spectral density and $R$ is the responsivity. This value is an indicator of the availability to detect the minimum illumination power; a low NEP is desirable for a photodetector.

\subsubsection{Specific Detectivity ( $\left.D^{*}, \mathrm{~cm} \mathrm{~Hz}^{1 / 2} \mathrm{~W}^{-1}\right)$}

The specific detectivity is a signal-to-noise ratio derived from the NEP and normalized by the optical area of the device to allow fair comparisons from detector to detector. Generally, this ratio is normalized by the optical area of the device to account for the variation from device to device. In this case, we consider the specific detectivity $\left(D^{*}\right)$, which can be expressed as $D^{*}=R A^{-1 / 2} / \delta / n$, where $A$ is the detector optical area, $R$ is the responsivity and $\delta / n$ is the current noise spectral density. The corresponding unit is $\mathrm{cm} \mathrm{Hz} z^{1 / 2} W^{-1}$, also known as Jones. The $D^{*}$ value depends on an applied bias and signal frequency. Because the main noise source is $1 / f$ noise, it may appear attractive to report detectivity at a high frequency. However, the responsivity measurement needs to be conducted at the same frequency. Typically, the detector needs to be characterized at frequencies lower than the $3 \mathrm{~dB}$ cutoff of its responsivity. A detectivity value should always be reported with the frequency used for its calculation, as well as the cutoff wavelength of the detector, operating temperature and bias.

\subsection{Response time}

As opposed to solar cells, for which the response time is not a relevant figure of merit, the response time of an IR sensor is critical. Sensors dedicated to imaging must be compatible with the video frame rate (i.e., exhibit a smaller response time than $1 / 25 \mathrm{~Hz}$ ). Other applications (missile detection, LIDAR detection) require a considerably higher response time $(<1 \mu \mathrm{s})$.

\subsubsection{Noise equivalent temperature difference (NETD, $m K$ )}

The NETD is a useful parameter to evaluate the performance of focal plane arrays (FPAs) influenced by the signal-to-noise ratio of IR detectors. This value quantizes the minimum temperature difference that can be imaged using an infrared sensor. This quantity is only relevant for thermal sensing (i.e., mostly for MWIR and LWIR applications) and can be expressed as ${ }^{351} \mathrm{NETD}=\delta \mathrm{n}_{\mathrm{n}} h V P_{\mathrm{in}} / k_{\mathrm{B}} \mathrm{T}_{\mathrm{B}}{ }^{2}$, where $\delta I_{\mathrm{n}}$ is the current noise spectral density, $\mathrm{P}_{\text {in }}$ is the incident optical power, $\mathrm{h}$ is Planck's constant, $v$ is the frequency of light, 
$k_{\mathrm{B}}$ is the Boltzmann constant, and $\mathrm{T}_{\mathrm{B}}$ is the blackbody radiation temperature. High performance quantum sensors reach NETDs of as low as $10 \mathrm{mK}$, while thermal sensors, such as bolometers, have typical NETD values in the 50 to $100 \mathrm{mK}$ range.

\subsection{Photoconductive devices}

\subsubsection{Conventional geometries}

HgTe is the most investigated material among mercury chalcogenide NCs for the following two reasons. The first reason is primarily cultural. In the field of IR detection, $\mathrm{HgCdTe}$ is the most investigated material, exhibiting a high performance and strong tunability from NIR to LWIR. As we switch from a bulk material to NCs, confinement plays the role of Cd alloying in the spectral response tuning. The second reason is related to the fact that among $\mathrm{HgX} \mathrm{NCs,} \mathrm{this} \mathrm{material} \mathrm{has} \mathrm{the} \mathrm{lowest} \mathrm{carrier} \mathrm{density,} \mathrm{which}$ considerably reduces the dark current, leading to higher performance. Progress on the device side is generally strongly correlated with the material development. Heiss and coworkers first reported an absorption of up to $3 \mu \mathrm{m},{ }^{27}$ and they promptly considered the potential of this material for photoconduction. ${ }^{1}$ The authors reported an elegant device based on an inkjet printed HgTe NC film. Using this approach, the authors drastically limited the amount of material required per device ( $2 \mu \mathrm{g}$ per device). The authors reported a high response (up to $4.4 \mathrm{~A} . \mathrm{W}^{-1}$ ) under extremely low power, suggesting the presence of electrical gain in the film, while the detectivity reached $3.2 \times 10^{10}$ Jones for $1.4 \mu \mathrm{m}$ peak detection at room temperature. Around the same period, Seong et al. ${ }^{2}$ also reported a NIR device based on small HgTe NCs. The authors designed a photoconductive device with a film of HgTe NCs connected by ITO electrodes. The material was designed to present absorption at $1.3 \mu \mathrm{m}$ corresponding to the current target for solar cell applications. At such wavelengths, HgTe NCs compete with lead chalcogenide NCs, which are more mature from both the synthesis and device perspectives. This fact is likely the reason why later, devices with longer wavelengths in the infrared region were considered to address a spectral range exempt from NC competitors. In particular, wavelengths above $2 \mu \mathrm{m}$ are challenging to address the use of lead chalcogenides because significantly large NCs with low colloidal stability are required. However, HgTe NCs remain small and exhibit their highest performance at such wavelengths. A critical breakthrough was obtained by Keuleyan et al., who realized the synthesis of HgTe NCs with sizes up to $12 \mathrm{~nm}$ and an absorption cutoff wavelength reaching 5 $\mu \mathrm{m}$ at room temperature. ${ }^{3}$ The authors designed a device in which a thin film of such NCs was deposited onto interdigitated electrodes and demonstrated the first midinfrared photoconduction in NC arrays. With an absorption edge above $3 \mu \mathrm{m}$, the proposed device potentially enabled thermal detection. Many advancements have been made to improve this basic photoconductive device, including the combination of several cutoff wavelengths in a device to design multicolor devices ${ }^{41,42,45}$ or the introduction of light resonators to enhance the light absorption. $37,39,40$ These devices are discussed later in this chapter. Because of its simplicity, the first progress related to photoconductive geometry correlated strongly with the material development. An interesting approach was proposed by Chen et al. ${ }^{36}$ The authors used surface chemistry based on $\mathrm{HgCl}_{2}$ and mercaptoethanol to achieve relatively high mobility films $\left(\approx 1 \mathrm{~cm}^{2} . \mathrm{V}^{-1} \mathrm{~s}^{-1}\right)$ and correlated this enhancement in mobility to the increased detectivity. This result is not notably important for the reported performance; however, the result highlights the requirement of a high film quality, as shown in Figure $37 \mathrm{~b}$.

Table 6 Figures of merit for photoconductive devices based on HgTe NCs. PC = Photoconductor, PT $=$ Phototransistor

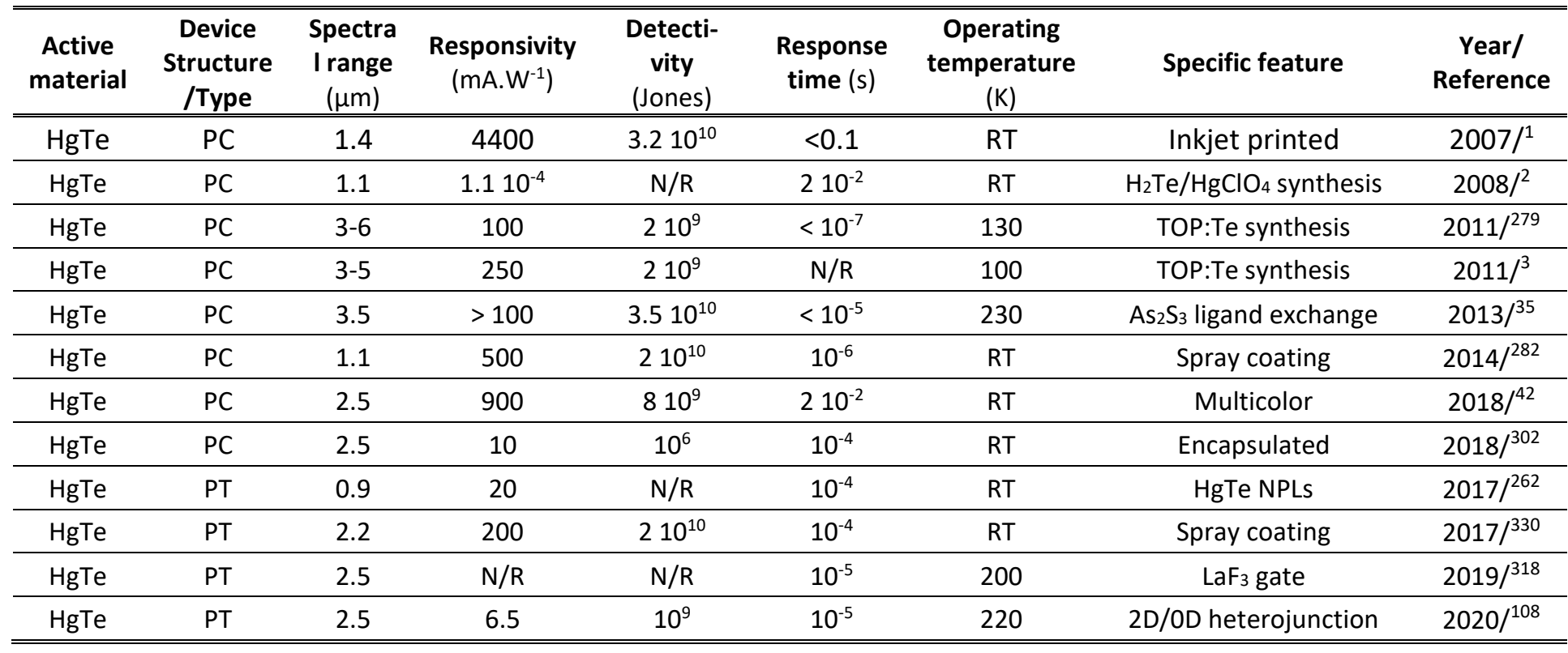




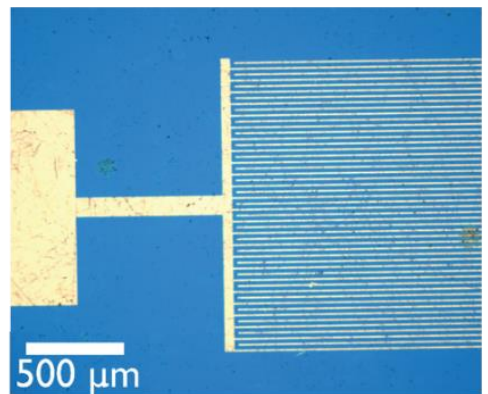

a.
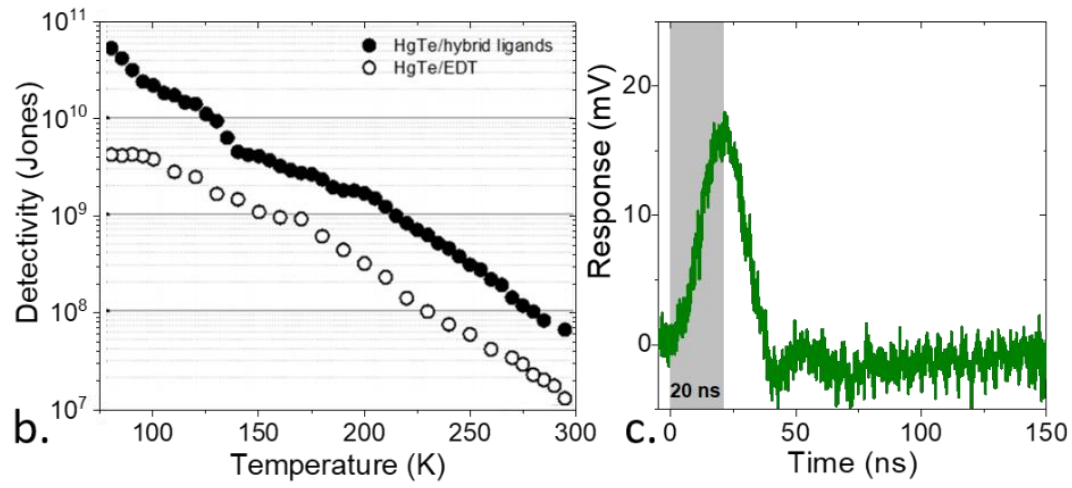

Figure 37 a. Microscopy image of interdigitated electrodes. b. Detectivity as a function of temperature for $\mathrm{HgTe}\left(\mathrm{HgCl}_{2} /\right.$ mercaptoethanol surface chemistry) and $\mathrm{HgTe} / \mathrm{EDT}$ films. Part $b$ is adapted with permission from ref ${ }^{36}$ Copyright (2019), American Chemical Society. c. Typical response of a $5 \mu \mathrm{m}$ cutoff wavelength film made of HgTe NCs to a $4.4 \mu \mathrm{m}$ pulsed quantum cascade laser. Part c is adapted with permission from ref ${ }^{219}$ Copyright (2018), American Chemical Society.

Interdigitated electrodes represent the most popular geometry for photoconductive devices; ${ }^{42,279,302}$ see Figure 37 a. This planar geometry is robust and can accommodate low quality films resulting from thickness fluctuations and pinholes. Films with this geometry are achieved using common deposition techniques, such as drop casting, ${ }^{35,279}$ spin coating, ${ }^{302}$ and spray coating. ${ }^{42,282,330}$ The interdigitated digits of the device act as a current amplifier. An advantage of photoconductive devices over their photovoltaic counterparts is the possibility of electrical gain. ${ }^{159,259,352,353}$ This process is often observed when one carrier has higher mobility than the other carrier. In this case, one carrier reaches the electrode faster than the other and is reinjected in the device to preserve the electrical neutrality of the film. Thus, a carrier (generated by a single photon) may contribute several times to the photocurrent. This aspect appears with a tradeoff of a reduced detector bandwidth (defined by the longest carrier lifetime). This phenomenon is commonly referred to as the gain/bandwidth product tradeoff in the electrical engineering community. This process is more efficient in small devices ( $<1 \mu \mathrm{m}$ and less), which is challenging to achieve with interdigitated geometry.

Nevertheless, photoconductive devices based on $\mathrm{HgTe}$ have been used to design photoconductive detectors covering the 1-5 $\mu \mathrm{m}$ range with a stronger focus on the SWIR/extended SWIR $(1.5-2.5 \mu \mathrm{m})$ and MWIR $(3-5 \mu \mathrm{m})$ ranges. Table 6 reviews the figures of merit associated with these devices. A responsivity of as high as a few A/W has been reported. However, responsivity on its own is an inferior figure of merit since it can be made arbitrarily high by increasing the bias and tuning the device geometry to generate a higher gain. The detectivity range is $10^{9}-10^{10}$ Jones at RT in SWIR, and an identical detectivity is obtained in MWIR under cryogenic conditions $(80-100 \mathrm{~K}$ ). Interestingly, most studies indicate that HgTe photoconductive devices present a fast time (ms and less) response. This aspect is in contrast with PbS NC-based devices, for which longer decay times are commonly observed in the transient photocurrent. Livache et al. demonstrated in MWIR (i.e., $5 \mu \mathrm{m}$ cutoff wavelength) HgTe NC device response times of as small as 20 ns under resonant excitation, ${ }^{219}$ as shown in Figure $37 \mathrm{c}$. 

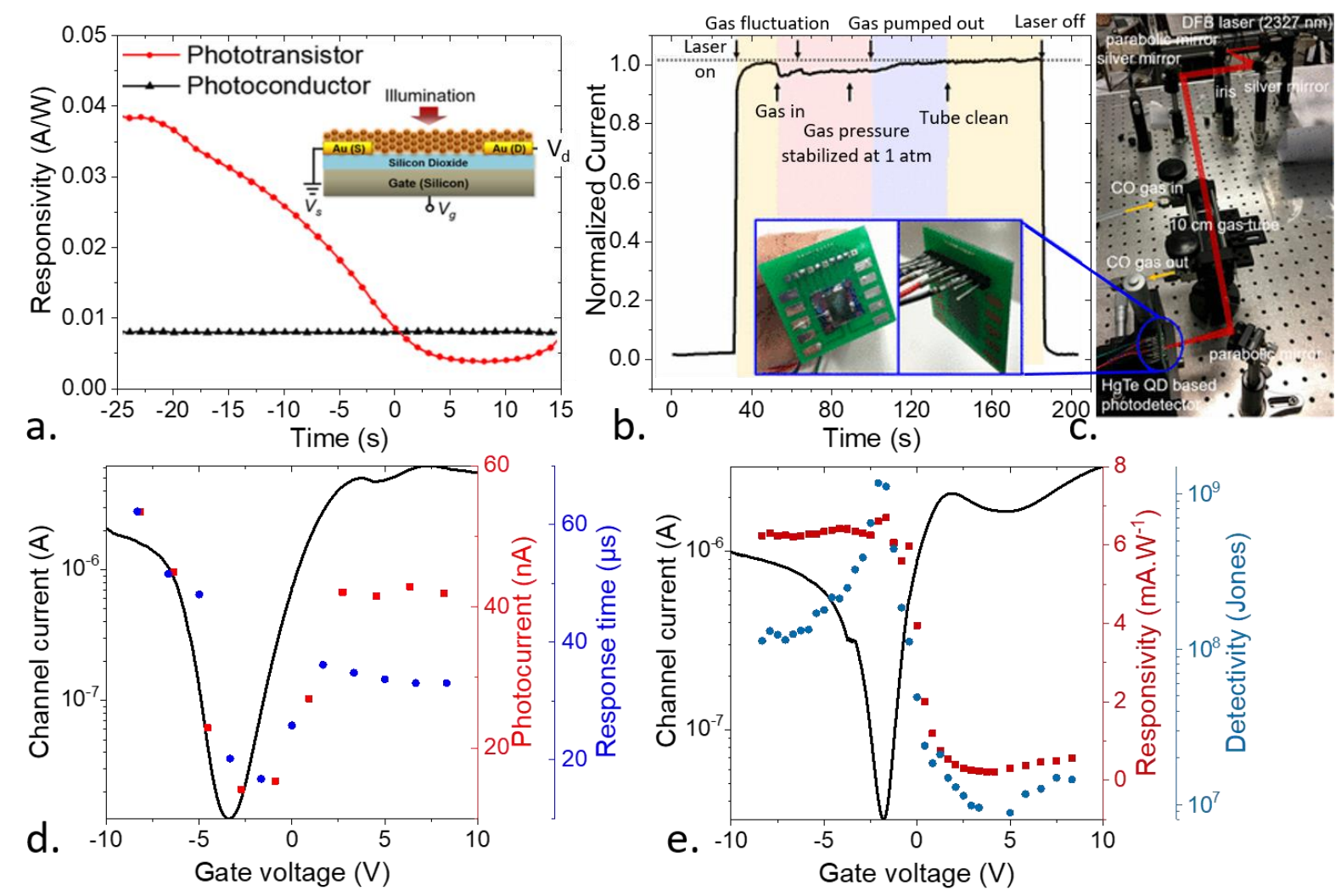

Figure 38 a. Gate voltage dependent responsivity of a phototransistor in comparison to its responsivity at floating gate operation. The inset shows a scheme of the device. $b$. Normalized room temperature photocurrent response of a $\mathrm{HgTe} Q \mathrm{QD}$-based phototransistor during the CO gas sensing experiment. $\mathrm{C}$. Picture of the experimental setup of the gas sensing system. Inset in b: Photos of the packaged phototransistor. Parts $a-c$ are adapted with permission from ref 330 Copyright (2017), American Chemical Society. d. Dark current, photocurrent and response time as a function of the applied gate bias for a phototransistor made of a HgTe NC film, whose gate is made of $\mathrm{LaF}_{3}$ ionic glass. Part d is adapted with permission from ref ${ }^{318}$ Copyright (2019), American Chemical Society. e. Dark current, photocurrent, and detectivity as a function of the applied gate bias for a phototransistor made of a $\mathrm{HgTe} \mathrm{NC}$ film with graphene electrodes with a gate made of $\mathrm{LaF}_{3}$ ionic glass. Part e is adapted with permission from ref ${ }^{108}$ Copyright (2020), American Chemical Society.

\subsubsection{Phototransistor}

A phototransistor is the successful combination of a photoconductive film and an FET. Applying a gate bias leads to additional tunability compared to bare photoconductors. A basic motivation for the phototransistor is the tunability of the photoresponse with the gate, as depicted in Figure 38a. Such devices have been applied mostly to HgTe NCs ${ }^{108,318,330}$ and NPLs. ${ }^{262}$ Chen et al. used such phototransistors for gas detection. The authors used the infrared range as a chemical fingerprint to detect $\mathrm{CO}$ (see Figure $38 \mathrm{~b}-\mathrm{c}$ ). Such applications are particularly interesting for NC-based IR sensors since the use of powerful laser sources alleviates the requirement for a low dark current. Nevertheless, the drift resulting from $1 / f$ noise is a major limitation.

Phototransistors made of NCs exhibit two main limitations. While the phototransitior geometry can enhance the device capability to tune the photoresponse, the dark current is usually tuned in a similar manner, and the two currents generally follow one another, ${ }^{262,318,330,354}$ as shown in Figure $38 \mathrm{~d}$. Consequently, applying a gate to increase the photoresponse increases the dark current. Alternatively, minimizing the dark current reduces the photoresponse of the device. In this case, the gate is not used to examine the nature of the majority carrier but rather as a key to prepare the material in a given carrier density state. In particular, the gate can be used to balance an initial doping of the film and reduce the dark current by exploiting the small offset of the dark current and photoresponse as a function of the applied gate bias. Consequently, this device is used as a patch to compensate for the limited material control at the synthesis level. The second limitation is from the response time; see Figure 38d. As the photoresponse is increased, the response is slowed. In other words, phototransistors operate at a quasiconstant gain bandwidth product. It may be interesting to slightly concede on the response time to generate an increased response, especially with $\mathrm{HgTe}$, which presents a fast response. The only exception to this behavior was reported by Numbe et al., who used graphene electrodes instead of ordinary metallic electrodes. ${ }^{108}$ Using this concept, the authors operated the transistor in a nonconventional mode, in 
which a large drain source bias was used to generate a p-n junction instead of uniform doping of the film. This concept has been extensively used to generate light from an FET, ${ }^{355-361}$ although its application to detection using NCs has been rare. By forming such gate controlled $p$ - $n$ junctions, the authors could identify the operating conditions under which the minimal dark conductance corresponded to the maximal photoresponse (see Figure 38e), thereby enhancing the overall detectivity of the device.

A current limitation of phototransistors is the film thickness. FETs based on surface gating methods can only tune the conductance of the film in the vicinity (typically over $\approx 50 \mathrm{~nm}$ ) of the gate interface. Such thin films cannot effectively absorb incident light, which limits the photoresponse of the device. Coupling the phototransistor to the resonators can likely help overcome the absorption tradeoff, this strategy has just been reported by coupling an STO gate with a metaling grating. ${ }^{339}$ The alternative approach is the use of electrolyte gating, which enables the gating of thick films. The method has been applied to the design of HgTe NPL-based phototransistors. ${ }^{262}$

\subsubsection{Flexible devices}

Among potential additional functions for infrared sensors based on $\mathrm{HgX} \mathrm{NCs,} \mathrm{the} \mathrm{concept} \mathrm{of} \mathrm{flexible}{ }^{362}$ substrates was introduced early for both FETs ${ }^{315,363}$ and detectors; ${ }^{42}$ see Figure 39a-b for an example of such a device. This effort mostly concerns $\mathrm{HgTe}^{304,364}$ but also works for $\mathrm{HgSe} \mathrm{NCs}^{186}$. In most reports, performances is tested as a function of the bending radius and number of cycles. However, the motivation for such flexible devices remains mostly unclear. Current developments for detection involve imaging applications, which require a readout circuit that is unlikely to be flexible. This aspect is even more unlikely if the device requires cooling with a Peltier or cryogenic machine. Nevertheless, one reports for a device based on a flexible substrate which was properly motivated. Tang et al. proposed the design of an eyelike detector; ${ }^{304}$ see Figure $39 c-e$ for a scheme of the concept. This work was initially motivated by the authors' strategy to conduct imaging using a single pixel detector with a scanning a lens in front of the detector to form an image. Nevertheless, despite the clear success of this approach, distortion occurs, and a curved detector is usually introduced to counterbalance the distortion introduced by the scanning method.

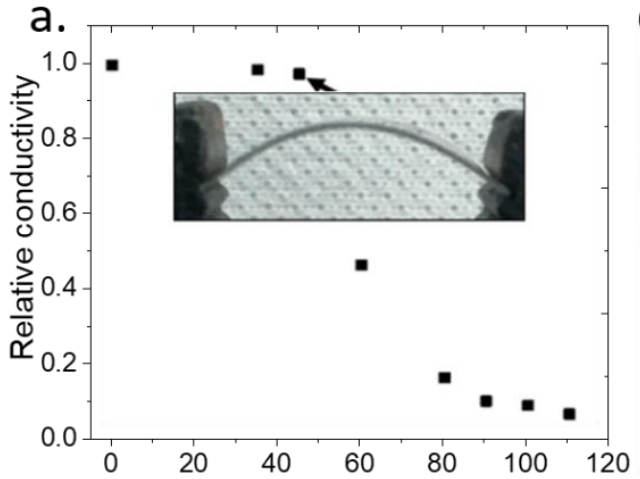

Device bend angle $\left({ }^{\circ}\right.$ from the horizontal) $\mathrm{d}$

b.

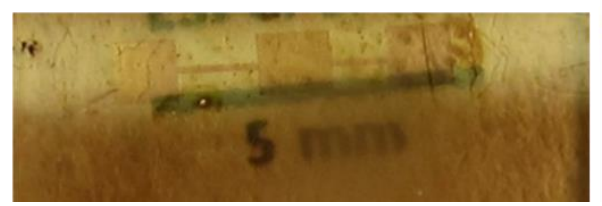

c.

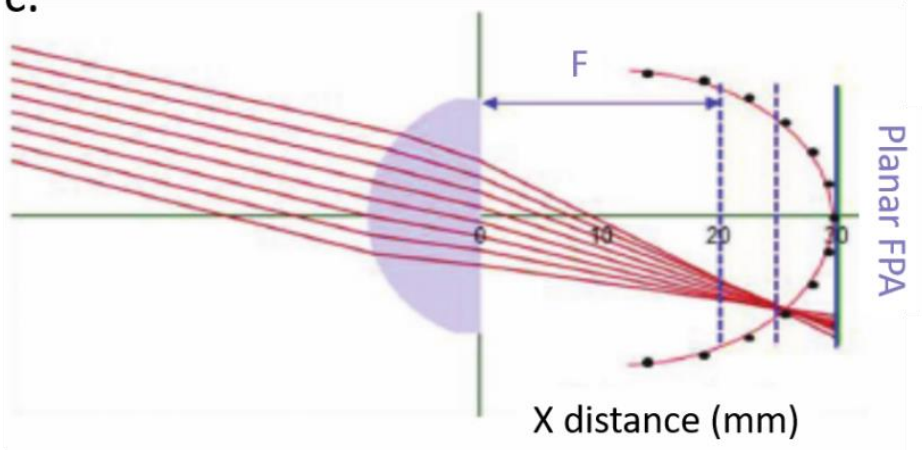

e.

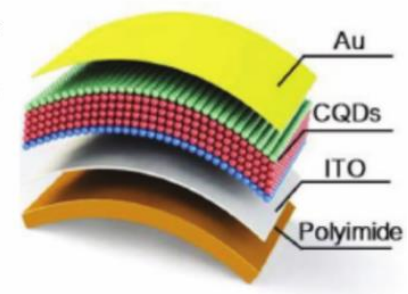

Figure 39 a. Plot showing the relative (dark) conductivity of the device after it was bent to the angle indicated. The inset is an image of the substrate bent at $45^{\circ}$. Part a is adapted with permission from ref ${ }^{42}$ Copyright (2018), American Chemical Society. b. Image of a flexible intraband detector based on $\mathrm{HgSe}$ NCs. Part b is adapted with permission from ref ${ }^{186}$ Copyright (2020), American Chemical Society. c. Ray tracing analysis of a single lens. The black dots and red curve denote the focal surface of the lens. d. Schematic of the ocular structure of humans. e. Illustration of the device architecture of flexible HgTe CQD photovoltaic detectors. Parts c-e are extracted from ref ${ }^{304}$. () 2019 WILEY-VCH Verlag GmbH \& Co. KGaA, Weinheim.

\subsection{Intraband detection}

While intraband transitions have been observed since the 1990s, the procedures used to induce such transitions (saturation of the interband transition ${ }^{365,366}$ or electrochemical gating ${ }^{332}$ ) were incompatible with practical device integration. Observation of 
the intraband in the steady state, ${ }^{65,66}$ because of self-doping in HgS, has been a breakthrough. ${ }^{33}$ Promptly after this observation, intraband photoconduction was investigated in $\mathrm{HgSe}^{29,34}$ and later in $\mathrm{HgTe}^{30,63,96}$ or $\mathrm{PbS}^{227}$ In these cases, photoexcitation occurs between the $1 \mathrm{~S}_{\mathrm{e}}$ and $1 \mathrm{P}_{\mathrm{e}}$ states in the conduction band. This transition is the $0 \mathrm{D}$ equivalent of the intersubband transition in doped quantum wells. In HgSe with particle sizes of approximately $5 \mathrm{~nm}$, this transition overlaps with the 3-5 $\mathrm{mm}$ range (MWIR); see Figure 40a.

This transition yields opportunities to develop an effective strategy for IR detection while reducing toxicity. Current narrow bandgap interband transitions are mostly observed using $\mathrm{Pb}$ - and $\mathrm{Hg}$-based materials and heavy atom containing materials. Narrow bandgaps can be more easily obtained from materials with a large number of electrons than from those with light atoms for which the density of the states is smaller. Thus, achieving doping in moderately doped nanocrystals may clarify intraband transitions in materials with reduced toxicity. Another key advantage of intraband transitions, compared with interband transitions in a semimetal, is the reduced sensitivity of the spectral response to sintering. In HgTe, owing to the lack of a bandgap and the extremely large Bohr radius $(40 \mathrm{~nm})$, annealing leads to a dramatic redshift of the optical bandgap. This aspect not only affects the detection cutoff wavelength but also increases the dark current. In this sense, the intraband transition appears to be more robust to mild annealing. However, it has been demonstrated that the carrier mobility is more sensitive to the size distribution in the case of intraband HgSe than that for interband HgTe NCs. ${ }^{121}$

However, many researchers were skeptical regarding the possibility of achieving high performance with intraband detection. Similar concerns were raised for intersubband transitions in doped quantum wells. The intraband transition is facilitated by doping, which also modifies the dark current. In doped particles, the photoinduced change in the carrier density is reduced compared to that in interband materials. This aspect, as well as the large dark current associated with these materials, explains why, to date, the detectivity of intraband detectors remains below $10^{9}$ Jones. ${ }^{57}$ The large dark current is not the only issue observed for an array of intraband nanocrystals. The photoresponse in HgSe arrays appears to be slow, with a response time of the order of seconds. This aspect raises concerns that the photoresponse may actually result from a bolometric effect rather than actual photoconduction. It has been claimed that a fast response time can be achieved when the doping magnitude corresponds to full filling of the $1 \mathrm{~S}$ state. ${ }^{34}$ This aspect appears to be practically difficult to achieve because postsynthesis ligand exchange also dramatically affects film doping. ${ }^{186}$ Finally, the dark current activation energy was found to be extremely small (20-40 meV), suggesting that sample cooling may not be able to reduce the dark current in the HgSe based detectors.

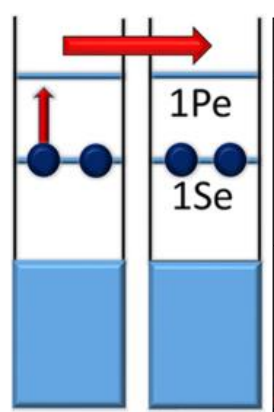

a.

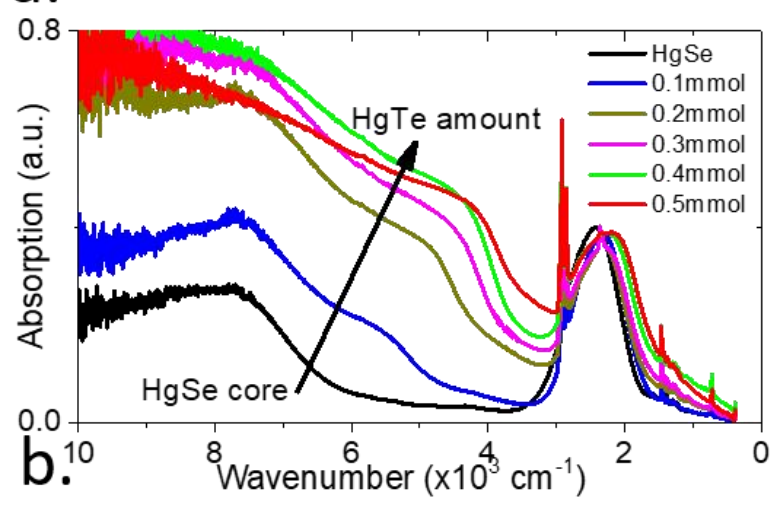

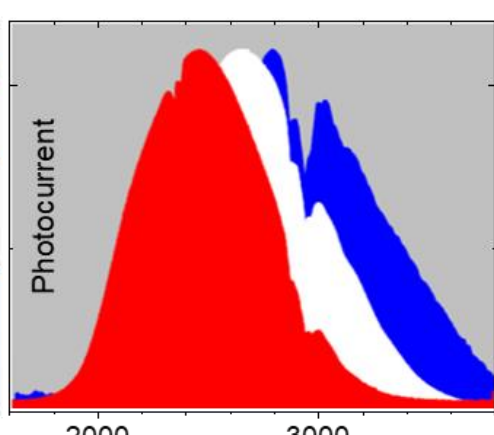

2000 3000

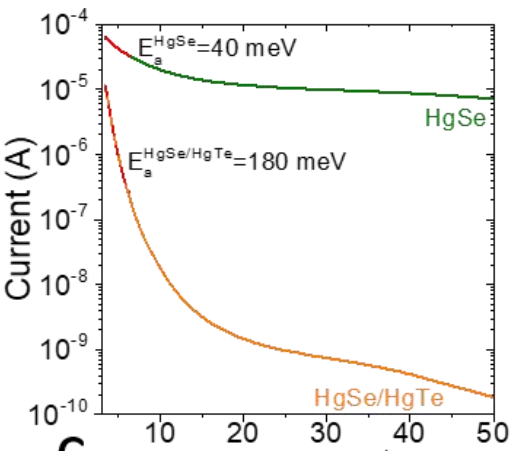

N C

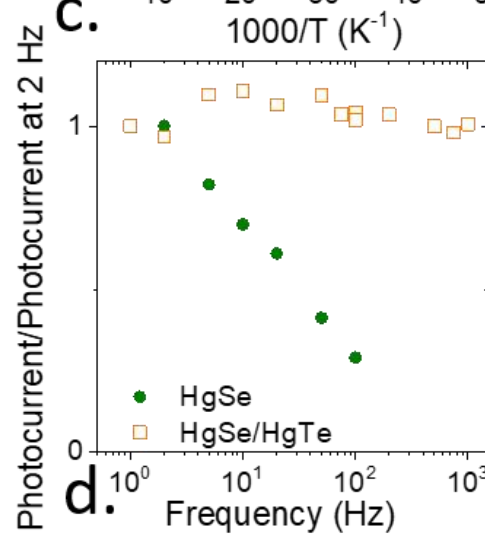

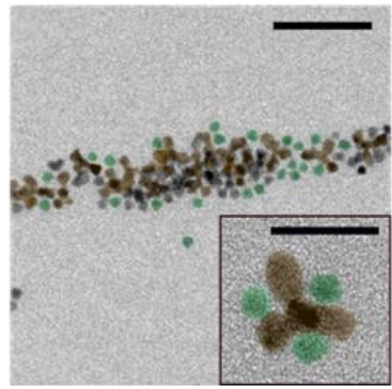

e.

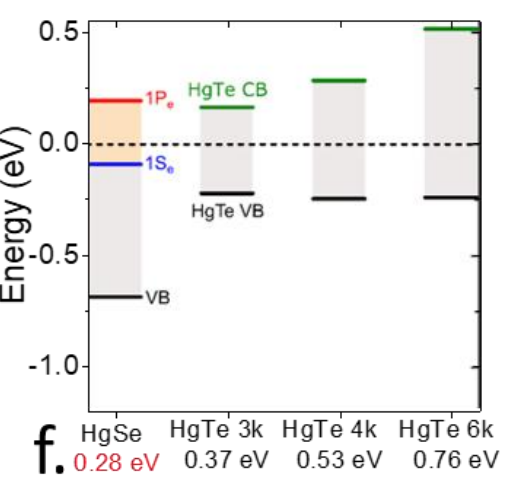

Figure 40 a. Principle of phototransport based on intraband photoconduction (left) and intraband photoconduction in an array of HgSe NCs with various sizes (right). Part a is adapted with permission from ref ${ }^{34}$ Copyright (2014), American Chemical Society. b. Absorption spectra of HgSe cores and $\mathrm{HgSe} / \mathrm{HgTe}$ core-shell structures for different amounts of HgTe. The intraband signal is barely affected by the addition of $\mathrm{HgTe}$, while the interband signal is amplified and redshifted. c. Current as a function of temperature for thin films made of $\mathrm{HgSe}$ cores and $\mathrm{HgSe} / \mathrm{HgTe}$ heterostructures. The Arrhenius fit 
close to the room temperature range is highlighted in red. $d$. Bode diagram (normalized signal magnitude at $2 \mathrm{~Hz}$ ) as a function of the signal frequency for thin films made of $\mathrm{HgSe}$ cores and a $\mathrm{HgSe} / \mathrm{HgTe}$ heterostructure. Parts $b-d$ are adapted with permission from ref ${ }^{120}$ Copyright (2014), American Chemical Society. e. TEM image of mixed HgSe and HgTe quantum dots for random colloidal QWIP fabrication. Certain HgSe (resp. HgTe) QDs are indicated in green (resp. brown). The scale bar is $50 \mathrm{~nm}$. Inset: higher resolution TEM image showing a HgTe tetrapod along with three HgSe spheres. The scale bar is $20 \mathrm{~nm}$. f. Electronic spectra of the four materials used for this study determined using a combination of XPS measurements and optical spectroscopy. The bandgap of each material is given on the $x$ axis (the intraband gap is given for HgSe). The black dashed line represents the Fermi level. Parts $e-f$ are taken from ref ${ }^{47}$.

To overcome these difficulties, the most recent strategies focus on heterostructures based on narrow bandgap materials, such as $\mathrm{HgSe}$ and HgTe. The driving concept was to couple the intraband absorption derived from HgSe with the interband (i.e., low dark current) transport properties from HgTe. The concept was inspired by III-V heterostructures, in which absorption and transport are spatially uncoupled. ${ }^{367-369}$ The first approach was proposed by Goubet et al. ${ }^{120}$ On a HgSe core, a coating of HgTe is colloidally grown (see Figure 11i-I). The addition of the HgTe layer does not considerably influence the energy of the intraband transition, while the interband absorption exhibits a strong redshift (see Figure 40b). Due to degenerate doping, the interband transition between the $1 \mathrm{~S}_{\mathrm{h}}$ and $1 \mathrm{~S}_{\mathrm{e}}$ states of $\mathrm{HgSe}$ is bleached due to the lack of an arrival state. However, the interband absorption coupling the valence band and the $1 \mathrm{Pe}$ state is still possible. The HgTe valence band is closer to vacuum than $\mathrm{HgSe}$, and its addition leads to a second path for interband absorption coupling of the valence band of $\mathrm{HgTe}$ with the $1 \mathrm{Pe}$ state of $\mathrm{HgSe}$. Owing to the relative position of the HgTe and HgSe valence bands, this absorption occurs at a lower energy, explaining the observed redshift. The introduction of HgTe increases the resistance of the sample, and the dark current activation energy reaches values higher than $150 \mathrm{meV}$ (Figure 40c), leading to a higher benefit of sample cooling. The intraband photoresponse was evaluated using a quantum cascade laser as a selective excitation source of the intraband transition. The authors observed a significant acceleration of the intraband response (Figure 40d). Using an alternative approach, the same group demonstrated that similar results could be obtained while eliminating the constraint of epitaxial growth between $\mathrm{HgSe}$ and $\mathrm{HgTe} .{ }^{47}$ The mixing of two solutions of HgSe NCs with intraband absorption and HgTe without intraband absorption (Figure 40e) yields similar results (higher resistance, higher activation energy, and faster response) while allowing more freedom for the metamaterial design. The bandgap of HgTe is thus a free parameter that can be optimized to favor charge transfer without generating dark current (see Figure 40f). Finally, the authors reported on the first colloidal intraband diode. The latter device is composed of a sapphire substrate, on which an Al grid is deposited and used as a partial midinfrared transparent electrode. A layer of a $\mathrm{HgSe} / \mathrm{HgTe}$ mixture with intraband absorption is coupled to a unipolar barrier made of HgTe NCs with a higher bandgap; see the scheme of the device in Figure 45h. The second electrode is made of gold. Interestingly, the diode exhibits a complex spectral photoresponse, in which the relative contribution of interband and intraband signals can be strongly modulated with the applied bias; see Figure 45i. Using this approach, the performance of intraband detectors has been enhanced to be similar to that of interband materials. This approach is promising, especially for longer wavelengths.

\subsection{Coupling to 2D Materials}

The concept of coupling nanocrystals with 2D materials was first proposed by Konstantatos et al. ${ }^{370}$ The motivation was to enhance the low mobility of nanocrystal arrays by coupling nanocrystals with graphene: the large carrier mobility of graphene $\left(\approx 1000 \mathrm{~cm}^{2}\right.$. $\mathrm{V}^{-1} \mathrm{~s}^{-1}$ at room temperature, while the NC arrays achieved values $\ll<1 \mathrm{~cm}^{2} . \mathrm{V}^{-1} \mathrm{~s}^{-1}$ ) was combined to the large spectral tunability of NC absorption. The design was first used to couple graphene with NIR PbS nanocrystals, typically for the design of NC-based solar cells. In such devices, absorption in the visible- and near-IR regions occurs mostly in the nanocrystals. Depending on the exact band alignment with graphene, one type of carrier is preferentially transferred to graphene, while the second carrier is trapped in the NC array. To ensure the neutrality of the whole structure, the first type of carrier is recirculated in graphene as long as the trapped carriers are not recombined, generating gain. The latter process is mostly driven by the recombination of these trapped charges with external molecules, which makes such devices sensitive to air and moisture. The recirculation process, also known as photoreduction gain, ${ }^{352}$ has been applied in the domain of nanocrystal arrays ${ }^{159,353}$ and was optimized due to the high mobility of graphene, which accelerated the recirculation process. Consequently, extremely large responsivities $\left(>10^{6} \mathrm{~A}\right.$. W-1 $\mathrm{W}^{-1} \mathrm{could}^{\mathrm{l}}$ be achieved. ${ }^{370,371}$ Following this result, the field of 0D/2D mixed dimensionality van der Waals heterostructures ${ }^{372}$ rapidly expanded ${ }^{373}$ and led to the use of other types of nanocrystals. ${ }^{374}$ The coupling of graphene with $\mathrm{HgTe}^{375}$ and $\mathrm{HgSe}^{376}$ nanocrystals was demonstrated by Tang et al., who considered the shift of the spectral response toward the midinfrared region; the scheme of this device is shown in Figure 41a. 

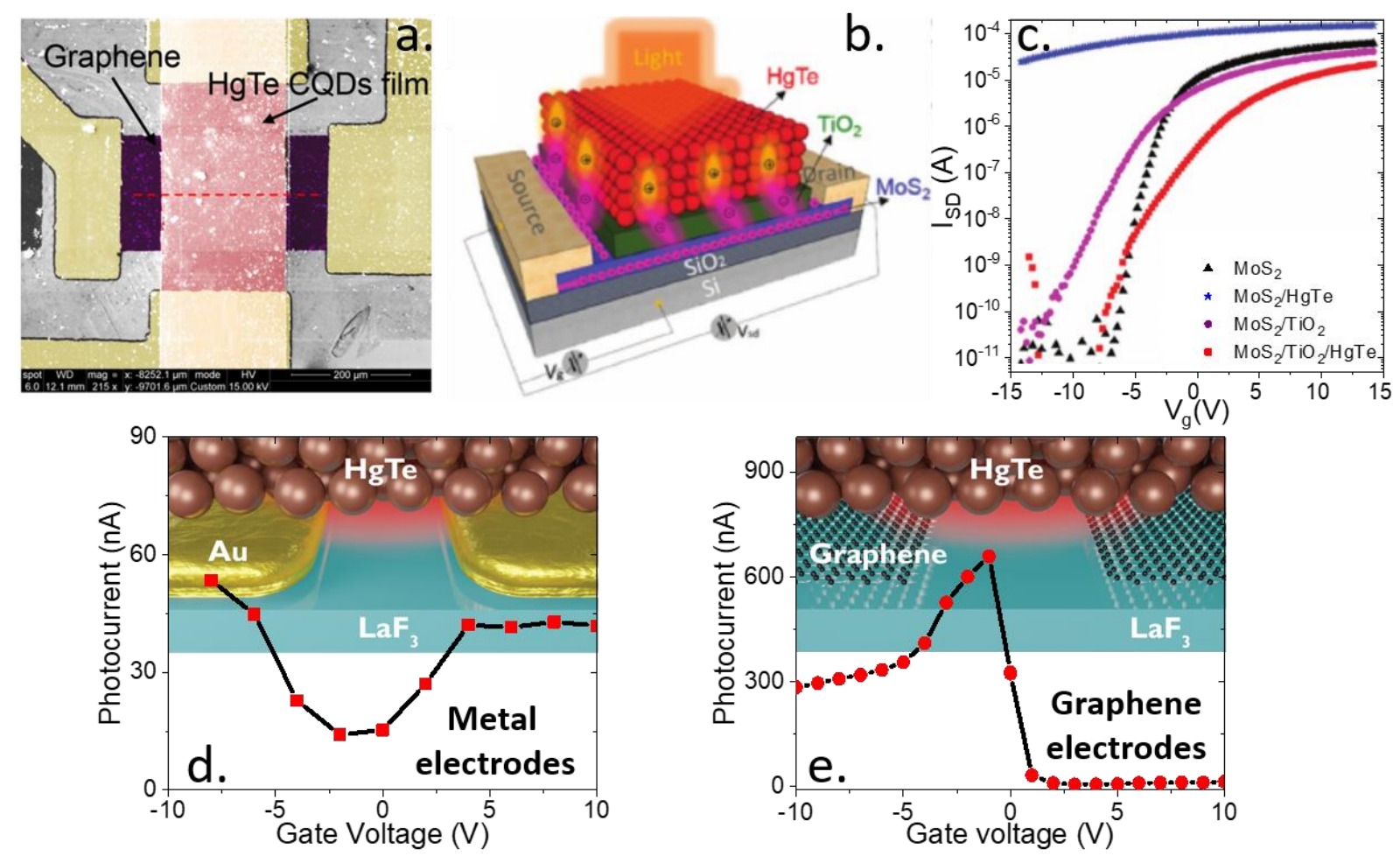

Figure 41 a. Faux color of a graphene/HgTe CQD junction. Part a is adapted with permission from ref ${ }^{375}$ Copyright (2019), American Chemical Society. b. Scheme of hybrid device under light illumination and photogenerated carried separation and transport within those photodetectors. $c$. Transfer curves (drain source current as a function of the fate current) of pristine $\mathrm{MoS}_{2}$ and $\mathrm{MoS}_{2} / \mathrm{HgTe}$ with and without the $\mathrm{TiO}_{2}$ from the photodetector devices presented in $b$. Parts $b$ and $c$ are reprinted with permission from ref ${ }^{377}$, (c) 2017 WILEY-VCH Verlag GmbH \& Co. KGaA, Weinheim. d. (resp. e.) Photocurrent as a function of the gate bias for gold/HgTe/gold (resp. graphene/HgTe/graphene) junction. The top part shows the scheme of the device. Parts $d$ and e are adapted with permission from ref ${ }^{108}$ Copyright (2020), American Chemical Society.

Despite this large response, graphene/QD heterostructures also suffer from certain key limitations. First, the high mobility of graphene is dramatically balanced with the lack of a bandgap. Indeed, the carrier density in graphene, despite being easily gate tunable, is larger than the thermally activated carrier density in a wide bandgap semiconductor. Although this claim is probably less true in regard to infrared materials, it results in a dark current and its associated $1 / f$ noise, ${ }^{378}$ which is large in graphene QD heterostructures. In addition, the response time of these heterostructures generally presents a slow component (of the order of seconds and sometimes, even hours), which is incompatible with the practical use of such devices. Last, the device presents an extremely low saturation with respect to the photon flux, and a high responsivity is only achieved for an extremely low irradiance. To reduce the carrier density, graphene was replaced by other 2D materials corresponding to a reasonable bandgap. For instance, graphene can be replaced by $\mathrm{MoS}_{2 .}{ }^{377}$ This transition metal dichalcogenide material has a slightly lower mobility, but the 2 eV bandgap allows it to overcome the dark current issues. As depicted in Figure 41c, the current tunability obtained with this gate is several orders of magnitude, compared to the factor of 2 to 5 obtained using graphene. Charge transfer between the conducting and absorbing layers can be further realized by the introduction of a $\mathrm{TiO}_{2}$ spacer (see Figure $41 \mathrm{~b}$ ).

The most recent ideas for $2 \mathrm{D} / \mathrm{HgX}$ devices do not focus on using 2D materials as high mobility transport layers but rather on other parts of the device, such as transparent conductive contacts to replace ITO or their gate tunable work function. Thus, Tang et al. proposed a dual color visible/SWIR detector combining a Si-based diode with a HgTe nanocrystal layer, in which the intermediate contact is graphene. ${ }^{43}$ Numbe et al. proposed the use of graphene as an infrared contact to design planar $p-n$ junctions, whose channel is made of a HgTe layer; see Figure $41 \mathrm{~d}$ and e. The authors attempted to exploit the large quantum capacitance of graphene. The authors demonstrated that a phototransistor made of a high capacitance ionic glass gate coupled to a HgTe absorbing layer exhibits an extremely different behavior when using metallic contact or graphene as electrodes. Because graphene is extremely thin, it enables the gate-induced electric field to propagate in the nanocrystal film, while the gating effect is completely screened in the case of gold electrodes. Consequently, the authors succeeded in generating a planar $p$ - $n$ junction that enhances the charge dissociation and boosts the detectivity by two orders of magnitude. ${ }^{108}$ 


\subsection{Photovoltaic devices}

Photovoltaic (PV) devices, typically based on vertical stacks, present the key advantage of a built in electric field, allowing for zero volt operation, leading to a large reduction in the dark current (and associated noise). ${ }^{301}$ Despite this key motivation, only a limited amount of work has been dedicated to this aspect. Such devices exhibit performances that are competitive compared to commercial devices, especially in the SWIR and extended SWIR ranges. ${ }^{48}$

Vertical geometry based infrared devices involve concerns regarding the design of transparent conductive electrodes and substrates. For visible and NIR ranges, the use of transparent conductive oxides (TCOs) is the most commonly implemented strategy. Tin-doped indium oxide (ITO) and fluorine-doped tin oxide (FTO) are the most commonly used TCOs. In the case of HgTe (work function $=4.6 \pm 0.1 \mathrm{eV}),{ }^{49,122,192} \mathrm{FTO}$ (work function $=4.4 \mathrm{eV}$ ) is better suited than ITO (work function $=4.7 \mathrm{eV}$ ) for use as an electron contact. However, FTO is typically deposited by chemical vapor deposition (CVD), which cannot be realized in most laboratories. Consequently, FTO is used as a bottom contact in a limited manner. Another limitation is the corresponding absorption in the infrared range, especially above $1 \mu \mathrm{m}$. A possible strategy to overcome this limitation is to reduce the TCO thickness ${ }^{302}$ at the price of a higher contact resistance. Above 2-2.5 $\mu \mathrm{m}$, glass absorption also becomes problematic. In this case, glass must be replaced with IR transparent materials, such as $\mathrm{CaF}_{2}{ }^{301}$ or sapphire ${ }^{303}\left(\mathrm{Al}_{2} \mathrm{O}_{3}\right)$. Among these materials, sapphire is an optimal candidate for low cost sensors, at least as long as the targeted wavelength remains below $5 \mu \mathrm{m}$. In the MWIR range, the use of a thin $(5 \mathrm{~nm})$ metallic layer was proposed as a transparent contact. However, thin metallic layers are not continuous, leading to high contact resistances. Thus, Tang et al. proposed the use of a thin gold layer coupled to a thin ITO layer to achieve both high transparency and low contact resistance. ${ }^{38}$ Livache et al. proposed another approach for the design of MWIR semitransparent (70\% transparency) conductive contacts with a metallic grid on top of a sapphire substrate. ${ }^{47}$ The contact design is expected to become an increasingly active field in the coming years, especially with the intense integration of resonators into devices to enhance light absorption. In this regard, resonators may reduce the constraint of transparency but raise the concern of plasmon losses.

The development of HgTe NC-based PV devices was initially motivated by the design of near-IR absorbers for solar cells. ${ }^{2,91,379,380}$ Extremely small HgTe NCs with the band edge at approximately the optimal value for a single junction solar cell have been grown and studied, and the design of vertical devices is clearly inspired by organic PV devices. Nam et al. ${ }^{379}$ proposed the use of HgTe NCs blended with a narrow bandgap electron donor polymer ${ }^{381}$ : poly[(4,4'-bis(2-ethylhexyl)dithieno[3,2-b:2',3'-d]silole)-2,6-diylalt-(2,1,3-benzothiadiazole)-4,7-diyl] (PSBTBT) to obtain a bulk heterojunction. A posteriori, the p-type nature of small HgTe NCs does not favor the creation of a bulk heterojunction by mixing the materials with an electron-donating polymer. Im et al. ${ }^{380}$ proposed a HgTe NC-based PV device inspired by solar cells, using HgTe NCs as absorbers for a dye-sensitized solar cell. ${ }^{382,383}$ The $\mathrm{HgTe}$ layer is sandwiched between a $\mathrm{TiO}_{2}$ layer and $\mathrm{P} 3 \mathrm{HT}$ layer used as an electron extractor and hole extractor, respectively. ${ }^{91}$ The presence of a polymer layer hinders the extension of the photoresponse of the device toward longer wavelengths due to the organic absorption. Consequently, Jagtap et al. later proposed a similar structure in which larger HgTe NCs were used to absorb light in the extended SWIR ${ }^{302}$, replacing the polymer layer with a thin inorganic transport layer of $\mathrm{MoO}_{3} .{ }^{192} \mathrm{However}$ the responsivity of the diode based on $\mathrm{FTO} / \mathrm{TiO}_{2} / \mathrm{HgTe} / \mathrm{MoO}_{3} / \mathrm{Au}$ remains weak. This aspect was attributed to the $\mathrm{TiO}_{2}$ layer, which can help induce a rectifying behavior to the $\mathrm{I}-\mathrm{V}$ curve $\mathrm{e}^{192,302}$ but also strongly filters the photogenerated electrons, leading to low responsivity $\left(<<m A . W^{-1}\right)$. 

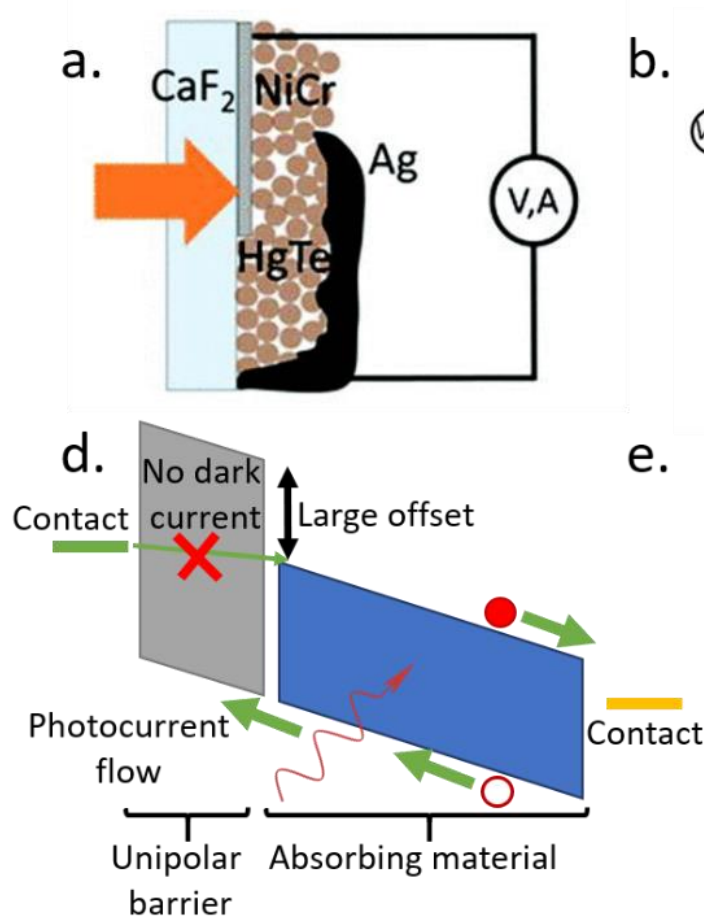

b.
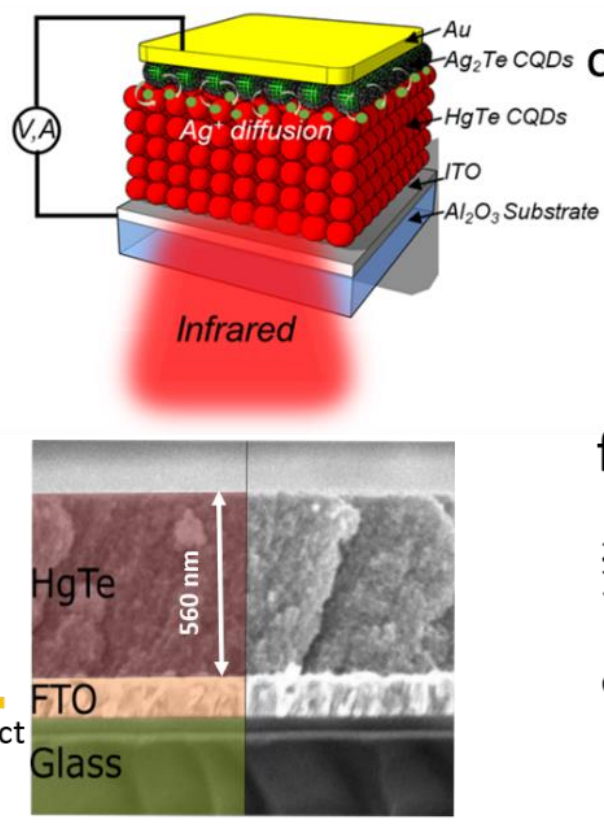
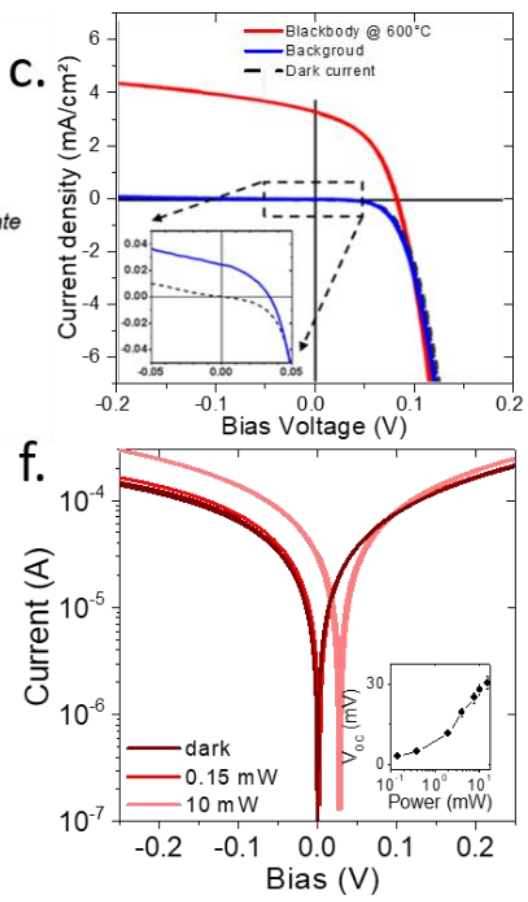

Figure 42 a. Schematic of a MWIR photovoltaic structure based on the $\mathrm{CaF}_{2} / \mathrm{NiCr} / \mathrm{HgTe} / \mathrm{Ag}$ structure. Reprinted from ref. ${ }^{301}$, with the permission of AIP Publishing. b. Schematic of a MWIR photovoltaic structure based on the $\mathrm{Al}_{2} \mathrm{O}_{3} / \mathrm{ITO} / \mathrm{HgTe} / \mathrm{Ag}_{2} \mathrm{Te} / \mathrm{Au}$ structure. c. Current density as a function of the bias voltage under dark conditions, background radiation, and $600{ }^{\circ} \mathrm{C}$ blackbody radiation at an operation temperature of $85 \mathrm{~K}$ of the diode presented in part $b$. The inset figure provides a zoomed in view of the dark current (closed shield) and background current (open shield). Parts $b$ and $c$ are adapted with permission from ref ${ }^{303}$ Copyright (2018), American Chemical Society. d. Band alignment associated with a unipolar barrier used to prevent dark current injection while allowing the photocurrent flow. e. SEM image of an extended SWIR photovoltaic structure based on the glass/FTO/HgTe $4 \mathrm{k} / \mathrm{HgTe}$ $6 \mathrm{k} / \mathrm{Au}$ structure. $\mathrm{f}$. I-V curves in the dark and under illumination by a $1.55 \mu \mathrm{m}$ laser at different powers for the diode shown in part e. Inset: evolution of open circuit voltage $\left(V_{O C}\right)$ as a function of increasing power. Parts e and $f$ are reprinted with permission from ref ${ }^{146}$. (C) 2019 WILEY-VCH Verlag GmbH \& Co. KGaA, Weinheim

Later, the use of wide bandgap layers for hole and electron extraction was avoided. HgTe and wide bandgap materials presented a large band alignment mismatch. Consequently, the wide bandgap led to barriers in both the valence and conduction bands. The layers filtered not only the dark current but also the photocurrent. Guyot Sionnest et al. ${ }^{301}$ proposed a Schottky diode in which the HgTe NCs were connected to contacts made of NiCr and Ag, with the latter contacts made of silver; see Figure 42a. Despite this simplistic fabrication, no electrical shortcomings were reported. This diode is the first reported to achieve background limited operation (In a background limited infrared photodetector (BLIP), the noise is limited by the fluctuation of the arriving photons).

Later, Ackerman et al. ${ }^{303}$ reported several key advancements in the design of HgTe NC-based PV devices; see Figure $42 \mathrm{~b}$. The most critical advancement is the introduction of a layer made of $\mathrm{Ag}_{2} \mathrm{Te} \mathrm{NCs}$ used as a hole extractor. This layer is exposed to the Hg precursor, which leads to a cation exchange process to form a strongly $p$-doped layer of $\mathrm{HgTe} / \mathrm{Ag}_{2} \mathrm{Te}$. The advantage of this structure is that it presents a clear diodelike transport behavior (see Figure $42 \mathrm{c}$ ) without using wide bandgap materials, which may filter the photocarriers. Later, the success of this structure was expanded to a bicolor diode ${ }^{46}$ and SWIR operation. ${ }^{48}$ To date, this structure leads to the highest performances (see Table 7). For electron extraction, the same group proposed the use of $\mathrm{Bi}_{2} \mathrm{Se}_{3}$ as a strongly $n$-type layer with a small bandgap, ${ }^{48,304}$ although this layer was eliminated in their recent work. ${ }^{48}$ This elimination was likely a result of the low colloidal stability of the large $\mathrm{Bi}_{2} \mathrm{Se}_{3}$ plates, which makes the fabrication of smooth films challenging. In parallel with the structure design, significant progress has been made in the surface chemistry of nanocrystals, switching from conventional solid state ligand exchange mostly conducted using ethanedithiol to liquid ligand exchange using a mixture of $\mathrm{HgCl}_{2}$ and short thiols (EDT or mercaptoethanol). ${ }^{36,146}$ In particular, Martinez et al. proposed the use of an ink in which NCs are dispersed in a polar solvent to realize the deposition of nanocrystals. In this case, the HgTe NCs capped with this mixture of ligands are phase transferred ${ }^{24}$ toward a polar solvent (DMF). Because of the high boiling point of DMF, it becomes critical to use highly concentrated solutions of NCs (> $250 \mathrm{mg} \cdot \mathrm{mL}^{-1}$ ) to facilitate the film drying. The key interest for this ink is to reduce the highly repetitive process 
of deposition/ligand exchange required by solid state ligand exchange, which often leads to the generation of holes or cracks in the film. In addition, inks with high concentrations enable the deposition of thick films (>300 $\mathrm{nm}$ ) in a single step; the cross-section of a device with a film thickness of approximately $600 \mathrm{~nm}$ is shown in Figure 42e. ${ }^{146}$ The increase in the film thickness over recent years has been a key aspect to increase the device absorption. Pushing the film thickness from $200 \mathrm{~nm}$ to $560 \mathrm{~nm}$ increases the absorption from $12 \%$ to $40 \%$ assuming a single pass of light in the absorbing layer.

To complete the discussion, a complementary approach for the design of HgTe NC-based PV devices relying on unipolar barriers must be mentioned. This concept was first developed for III-V heterostructures 384,385 and is depicted in Figure $42 \mathrm{~d}$. The absorbing layer is coupled to a barrier that is used to prevent dark current injection while allowing the photocurrent to flow. To achieve this goal, a specific band alignment is required: the photocarrier conducting band of the active material must be resonant with that of the barrier, while the other band must feature a large offset, preventing the dark current. Such a design requires an accurate understanding of the band offset between the two materials, which is possible for III-V semiconductors but challenging for II-VI semiconductors. The band alignment results from the inverted band structure, quantum confinement, and surface chemistry. To this end, Jagtap et al. conducted a systematic study of the band alignment in HgTe NCs as a function of the size and surface chemistry (see Figure 23b and c). The authors observed that the valence band offset for various sizes of HgTe is small and most of the confinement energy is carried by the shift of the conduction band. This observation reflects the higher effective mass of the valence band compared with that of the conduction band; see the dispersion diagram in Figure 14c or Figure 15b. Consequently, a thin layer made of smaller HgTe NCs (i.e., higher bandgap) behaves as a unipolar barrier (no offset for valence band, large offset for conduction band) for larger NCs. Jagtap et al. ${ }^{192}$ introduced a thin layer of HgTe with a $6000 \mathrm{~cm}^{-1}(\approx 720 \mathrm{meV}$ or $\approx 1.8 \mu \mathrm{m})$ band edge energy in a device in which the absorbing layer is made of HgTe with a $4000 \mathrm{~cm}^{-1}$ bandgap $(\approx 480 \mathrm{meV}$ or $\approx 2.5 \mu \mathrm{m}$ ). The introduction of this thin layer leads to diode behavior for the I-V curve, with the presence of an open circuit voltage under illumination ${ }^{47,146}$ at room temperature (see Figure 42f). This barrier also increases the detectivity by a factor of $10 .{ }^{192}$

To date, PV devices have exhibited the highest detection performance. Advancements have been made by combining the developments in surface chemistry and PV device design. In the next section, we discuss how the introduction of resonators has enhanced the performance due to better light-matter coupling and enhancement of device absorption. Currently, the design of energy band profiles suffers from a lack of rationality. Thus, optimal electrical properties might be realized in the future. The other key challenge relative to PV devices is the transfer of the diode structure toward FPAs. For example, gold is used as a contact in most devices, even though this metal is not CMOS compatible. A systematic investigation of the effect of the contact work function must be conducted in the future.

Table 7 Figures of merit associated with photovoltaic infrared devices based on HgX NCs. RT=Room temperature

\begin{tabular}{|c|c|c|c|c|c|c|c|c|}
\hline $\begin{array}{l}\text { Active } \\
\text { material }\end{array}$ & $\begin{array}{l}\text { Device } \\
\text { Structure } \\
\text { /Type }\end{array}$ & $\begin{array}{l}\text { Spectral } \\
\text { range } \\
(\mu \mathrm{m})\end{array}$ & $\begin{array}{l}\text { Respon } \\
\text { sivity } \\
\text { or EQE } \\
\text { (mA.W } \\
{ }^{1} \text { or } \% \text { ) }\end{array}$ & $\begin{array}{l}\text { Detec } \\
\text { tivity } \\
\text { (Jone } \\
\text { s) }\end{array}$ & $\begin{array}{l}\text { Respon } \\
\text { se time } \\
\text { (s) }\end{array}$ & $\begin{array}{l}\text { Operati } \\
\text { ng } \\
\text { temper } \\
\text { ature } \\
\text { (K) }\end{array}$ & Specific feature & $\begin{array}{l}\text { Year/ } \\
\text { Reference }\end{array}$ \\
\hline HgTe & $\begin{array}{l}\mathrm{FTO} / \mathrm{TiO}_{2} / \mathrm{HgTe} / \mathrm{P} 3 \mathrm{HT} / \\
\mathrm{Au}\end{array}$ & $\begin{array}{l}\mathrm{NiR} \\
0.8-1.4 \\
\end{array}$ & $1.3 \%$ & - & $\approx \mathrm{ms}$ & RT & $\begin{array}{l}\text { Depleted } \\
\text { heterojunction }\end{array}$ & $2012 /^{380}$ \\
\hline HgTe & $\begin{array}{l}\text { ITO/PEDOT:PSS/HgTe: } \\
\text { PSBTBT/AI }\end{array}$ & $\begin{array}{l}\text { Vis-NiR } \\
0.4-1.1\end{array}$ & $1.4 \%$ & - & - & RT & Bulk heterojunction & $2014 /{ }^{379}$ \\
\hline $\mathrm{HgTe}$ & $\mathrm{ITO} / \mathrm{TiO}_{2} / \mathrm{HgTe} / \mathrm{Au}$ & E-SWIR & $5 \times 10^{-3}$ & $\approx 10^{7}$ & $\approx \mu \mathrm{S}$ & RT & $\begin{array}{l}\text { Air stable via device } \\
\text { encapsulation }\end{array}$ & $2018 /{ }^{302}$ \\
\hline HgTe & $\begin{array}{l}\mathrm{FTO} / \mathrm{TiO}_{2} / \mathrm{HgTe} / \mathrm{HgTe}- \\
\text { p type } / \mathrm{MoO}_{3} / \mathrm{Au}\end{array}$ & E-SWIR & 0.2 & $3 \times 10^{8}$ & $\approx \mu \mathrm{s}$ & RT & Unipolar barrier & $2018 /{ }^{192}$ \\
\hline HgTe & $\begin{array}{l}\text { FTO/HgTe/HgTe p- } \\
\text { type/Au }\end{array}$ & E-SWIR & 20 & $3 \times 10^{9}$ & $370 \mathrm{~ns}$ & RT & $\begin{array}{l}\text { Thick film made from } \\
\text { ink }\end{array}$ & $2019 /{ }^{146}$ \\
\hline HgTe & $\begin{array}{l}\text { Polyimide } / \mathrm{ITO} / \mathrm{Bi}_{2} \mathrm{Se} \\
{ }_{3} / \mathrm{HgTe} / \mathrm{Ag}_{2} \mathrm{Te} / \mathrm{HgTe} / \\
\mathrm{Au}\end{array}$ & SWIR & 220 & $\begin{array}{l}7.5 \times \\
10^{10}\end{array}$ & $260 \mathrm{~ns}$ & RT & Flexible substrate & $2019 / 304$ \\
\hline HgTe & $\begin{array}{l}\mathrm{Al}_{2} \mathrm{O}_{3} / \mathrm{ITO} / \mathrm{HgTe} / \mathrm{Ag}_{2} \mathrm{Te} \\
/ \mathrm{Au}\end{array}$ & SWIR & $\approx 1000$ & $\approx 10^{11}$ & $1.4 \mu \mathrm{s}$ & RT & $\begin{array}{l}\text { High detectivity at } \mathrm{RT} \text {, } \\
\text { crosslinking } \\
\mathrm{EdT} / \mathrm{HCl}\end{array}$ & $2020 / 48$ \\
\hline
\end{tabular}




\begin{tabular}{|c|c|c|c|c|c|c|c|c|}
\hline HgTe & $\begin{array}{l}\mathrm{LaF}_{3} / \text { Graphene gate } \\
\text { (FET) }\end{array}$ & E-SWIR & 1 & $10^{9}$ & $<10 \mu \mathrm{s}$ & 220 & $\begin{array}{l}\text { Gate induced } p-n \\
\text { junction }\end{array}$ & $2020 /{ }^{108}$ \\
\hline HgTe & $\mathrm{CaF}_{2} / \mathrm{NiCr} / \mathrm{HgTe} / \mathrm{Ag}$ & MWIR & 38 & $10^{10}$ & $0.7 \mu \mathrm{s}$ & 140 & BLIP detection & $2015 / 301$ \\
\hline HgTe & $\begin{array}{l}\mathrm{Al}_{2} \mathrm{O}_{3} / \mathrm{ITO} / \mathrm{HgTe} / \mathrm{Ag}_{2} \\
\mathrm{Te} / \mathrm{Au}\end{array}$ & MWIR & $\begin{array}{r}\approx 100 \\
380\end{array}$ & $\begin{array}{l}3 \times 10^{9} \\
\approx 10^{11}\end{array}$ & $\begin{array}{l}20 \mu \mathrm{s} \\
\approx 1 \mu \mathrm{s}\end{array}$ & $\begin{array}{l}\text { @RT } \\
@ 85\end{array}$ & $\begin{array}{l}\text { Doping by contact } \\
\text { layer, high detectivity }\end{array}$ & $2018 /{ }^{303}$ \\
\hline HgTe & $\begin{array}{l}\mathrm{Al}_{2} \mathrm{O}_{3} / \mathrm{ITO} / \mathrm{Bi}_{2} \mathrm{Se}_{3} / \mathrm{Hg} \\
\mathrm{Te} / \mathrm{Ag}_{2} \mathrm{Te} / \mathrm{HgTe} / \mathrm{Au}\end{array}$ & $\begin{array}{l}\text { SWIR } \\
\text { MWIR }\end{array}$ & 400 & $\begin{array}{l}3 \times 10^{1} \\
0 \\
\approx 10^{11}\end{array}$ & $2.5 \mu \mathrm{s}$ & 85 & bicolor SWIR/MWIR & $2019 /{ }^{46}$ \\
\hline $\begin{array}{l}\text { HgTe:Hg } \\
\text { Se }\end{array}$ & $\begin{array}{l}\mathrm{Al}_{2} \mathrm{O}_{3} / \mathrm{Al} / \mathrm{HgTe} / \mathrm{HgSe} \\
: \mathrm{HgTe} / \mathrm{Au}\end{array}$ & $\begin{array}{l}\text { MWIR- } \\
\text { SWIR }\end{array}$ & $\approx 5$ & $\approx 10^{9}$ & $200 \mathrm{~ns}$ & 80 & $\begin{array}{l}\text { Quantum well like } \\
\text { structure with hybrid } \\
\text { CQD. } \\
\text { Intraband detection }\end{array}$ & $2019 /{ }^{47}$ \\
\hline
\end{tabular}

\subsection{Enhanced light matter coupling}

In NC arrays, achieving decent photoconduction comes at a cost. Due to hopping transport, the mobility in NC arrays is limited. Although significant progress $s^{29,35,194,317}$ has been made in the direction of increased mobility values ${ }^{4,24,386}$ from below $10^{-4}$ to approximately $1 \mathrm{~cm}^{2} \mathrm{~V}^{-1} \mathrm{~S}^{-1}$ using ink-based films with hybrid passivation (ions and organics) to passivate different facets, the value of mobility remains two to three orders of magnitude below the bulk values. Consequently, the charge diffusion length extends over several NCs (10-100 nm) but remains shorter than the absorption depth (few $\mu \mathrm{m})$ in such films. As discussed earlier in this review, films with thicknesses of a few micrometers are required to absorb most of the incident light. For instance, a $200 \mathrm{~nm}$ thick film, which is the typical thickness of films obtained from solid state ligand exchange, with a band edge at $2.5 \mu \mathrm{m}(0.5 \mathrm{eV}$ or 4000 $\mathrm{cm}^{-1}$ ) absorbs only $12 \%$ of the incident light for a single pass of the light. The tradeoff is a result of the fact that building thicker films is not useful since the charges cannot be collected.

Two approaches can be used to overcome these severe limitations. The first approach is to work on the material side to fabricate higher quality (i.e., mirror like, with a film roughness of the order of the NC size) thick (> $500 \mathrm{~nm}$ ) films with improved internanocrystal coupling (i.e., higher $\mu$ ). Such efforts have been conducted considering the design of photodiodes in vertical geometry, ${ }^{303}$ and $\mathrm{HgTe}$-based inks have been used to facilitate thick film deposition with a reduced number of steps. ${ }^{36,146}$ The second method to overcome the diffusion length/absorption depth tradeoff is to control the light-matter coupling within the NC film. The driving concept is to modify the electromagnetic field to concentrate it into the absorbing layer. Resonators can be introduced, enabling an increase in the magnitude of the incident field in a thin slab of the absorbing semiconductor, resulting in an increase in absorption. The field enhancement can be considered to be a reduction of the effective absorption depth to eliminate the previously mentioned tradeoff. To date, most efforts have been focused in this direction. Another aspect that has not been extensively examined for NCs is the planar focusing of the light to uncouple the optically absorbing area of the device with its electrical area. The concept is to collect photons over an area that is as large as possible while reducing the electrical area as much as possible, thereby proportionately decreasing the dark current. ${ }^{387}$ Zhu et al. recently demonstrated ${ }^{388}$ a device based on a silicon photonic waveguide with a small volume of HgTe used as an IR absorbing material. This structure shows an enhanced detectivity compared to the bare HgTe film.

The coupling of resonators with $\mathrm{HgX} \mathrm{NC}$ films is, for most devices, based on the introduction of plasmonic resonators. The first demonstration was made by Zhao and coworkers. The authors coupled gold nanorods to absorbing films of HgTe NCs, ${ }^{389}$ as shown in Figure 43a. Gold nanorods present two resonances, and the resonance associated with the long direction of the nanorods falls in the near-infrared region (see Figure 43b). Small HgTe NCs were synthesized to match this wavelength. To avoid exciton quenching in the gold, a $\mathrm{ZnO}$ spacer was deposited on top of the gold nanorods. By using this approach, the photocurrent is enhanced by a factor of 2. Following this work, various approaches to couple HgX NC films with plasmonic resonators have been demonstrated using a top-down approach for the fabrication.

Yifat et al. ${ }^{37}$ used a photoconductive device consisting of interdigitated electrodes coupled to HgTe absorbing films in the MWIR range. Using e-beam lithography, the authors functionalized the substrate with an array of gold nanorods. The authors matched the resonance with the HgTe band edge and observed an increase in the photocurrent up to a factor of 3 . Because the authors used gold rods as a pattern, the photocurrent was polarized and observed only for light polarization along the rods. The same geometry was later applied by Tang et al. to $\mathrm{HgSe}^{39}$, for which the resonance matched the intraband absorption in $\mathrm{HgSe}$. To avoid the polarization effect, the authors used disks instead of rods as a pattern. Chu et al. proposed another version of this device. . $^{40,337}$ In previous devices, transport was supported by interdigitated electrodes and was completely uncoupled from the array of resonators. Chu et al. combined these electrical contacts and optical resonance in the same set of electrodes; see Figure $43 \mathrm{j}$. In 
other words, the period and size of the interdigitated electrodes were used to induce resonance. A second improvement is the introduction of a backside mirror to induce a second pass of the light through the absorbing layer. This resonator is known as the guided mode resonator (GMR) because it reshapes the incident plane wave into a steady wave propagating along the substrate in the NC film. Because of the interdigitated electrode pattern, the field enhancement was polarized, and the two resonances were used to broaden the spectral response (see Figure $43 \mathrm{~h}$ ). The design obtained $\approx 100 \%$ absorption along one polarization ( $70 \%$ of the total signal). The other key advantage of merging plasmonic resonators with transport electrodes was the generation of gain. Because the electrode spacing was reduced, the transit time in the structure was also reduced, which generated electrical gain. Consequently, by using these GMR electrodes, the photocurrent enhancement was considerably higher than the absorption enhancement, reaching a factor of approximately 1000 (see Figure 43i). Finally, the authors demonstrated that a clear tunability of the spectral response can be attained by tuning the period of the grating. In this sense, this geometrical tunability, through the period, offers a new postsynthesis degree of freedom for the design of the photodetector in addition to that due to the quantum confinement in the material. Tang et al. proposed this method for vertical geometry devices. ${ }^{38}$ In this case, the authors added an array of gold nanodisks on top of the ITO electrode (see Figure 43d) in a diode structure reported previously. ${ }^{303}$ The photocurrent enhancement reached a factor of 3-4 (see Figure 43c).

Plasmonic resonators have been successful; however, these devices introduce several losses in the metal and ITO electrodes. For example, in the GMR electrode proposed by Chu et al., $\approx 25 \%$ of the absorption occurs in the electrodes and does not generate a photocurrent. Other types of resonators have been proposed, including Fabry-Perot resonators; ${ }^{303,304}$ see Figure $43 \mathrm{f}$. In this case, a dielectric spacing layer is required, which leads to several resonances due to the harmonic behavior; see Figure 43g. A distributed feedback mirror cavity has also been proposed by the same group. In this case, rather than the enhancement of the photocurrent, the spectral shaping of the photoresponse is targeted; ${ }^{44}$ see Figure $45 \mathrm{~g}$.

In all these reports, fabrication of the resonators was performed before the deposition of the HgX nanocrystal film. Such an approach accounts for the fragility of $\mathrm{HgX}$ nanocrystals, which easily sinter when annealed or exposed to nonsolvents. Thus, most of the groups choose a strategy in which all the lithography steps are performed at the early stage of fabrication and $\mathrm{HgX} \mathrm{NCs} \mathrm{are}$ deposited as a top layer or require only minimal postdeposition processing (top metal contact). An exception was reported by Tang et al. last year. ${ }^{390}$ The grating generating the optical features was directly implemented by stamping the HgTe film.

The coupling of $\mathrm{HgX}$ films to resonators has been comparatively successful over recent years, and this direction must be pursued. The losses within the metallic parts of the resonator must be addressed, likely through the introduction of dielectric resonators. Another promising direction is to supplement the introduction of a single resonance. In solar cells, up to 9 resonances can be implemented to achieve broad band absorption. ${ }^{391}$ 

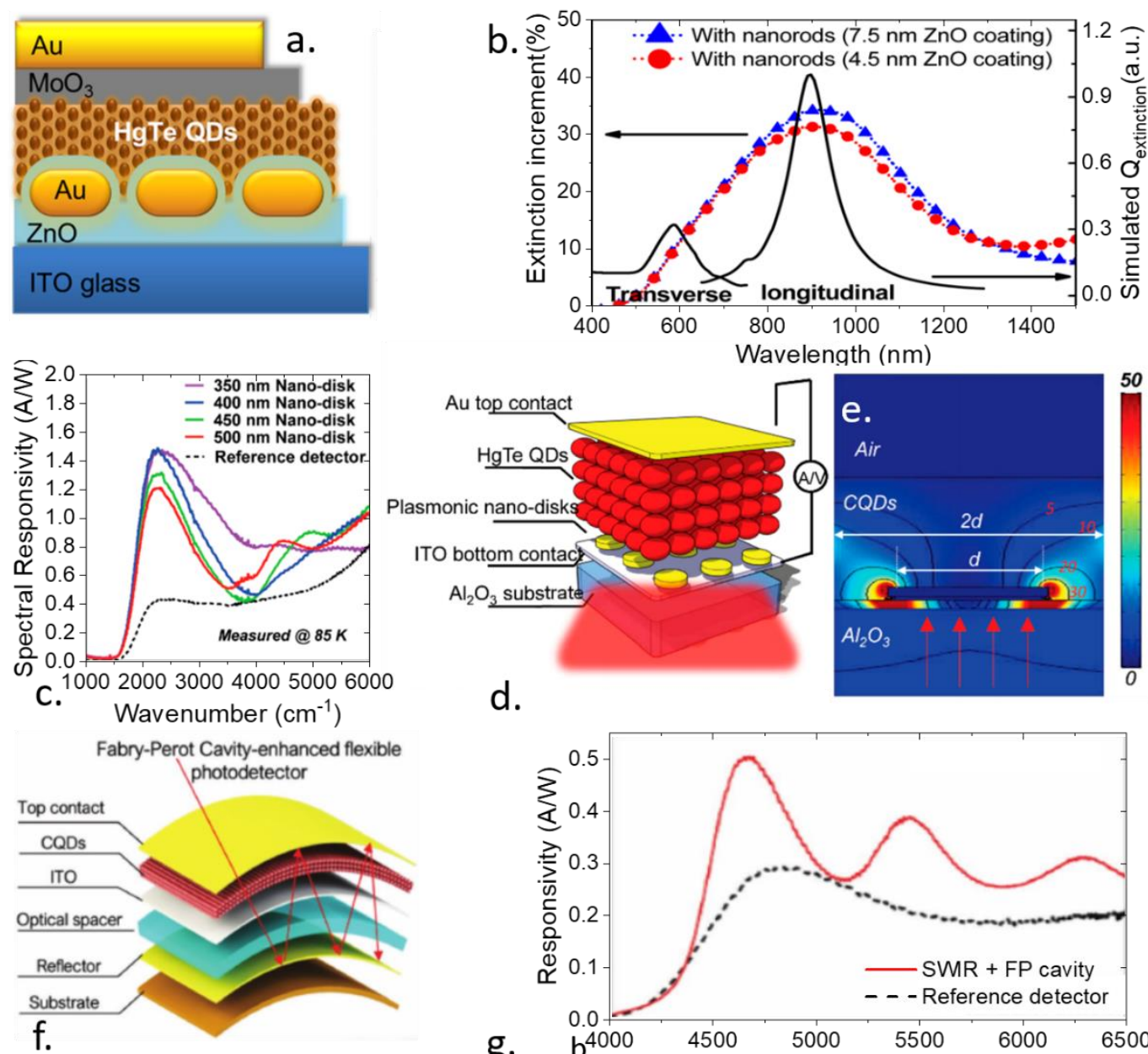

d.
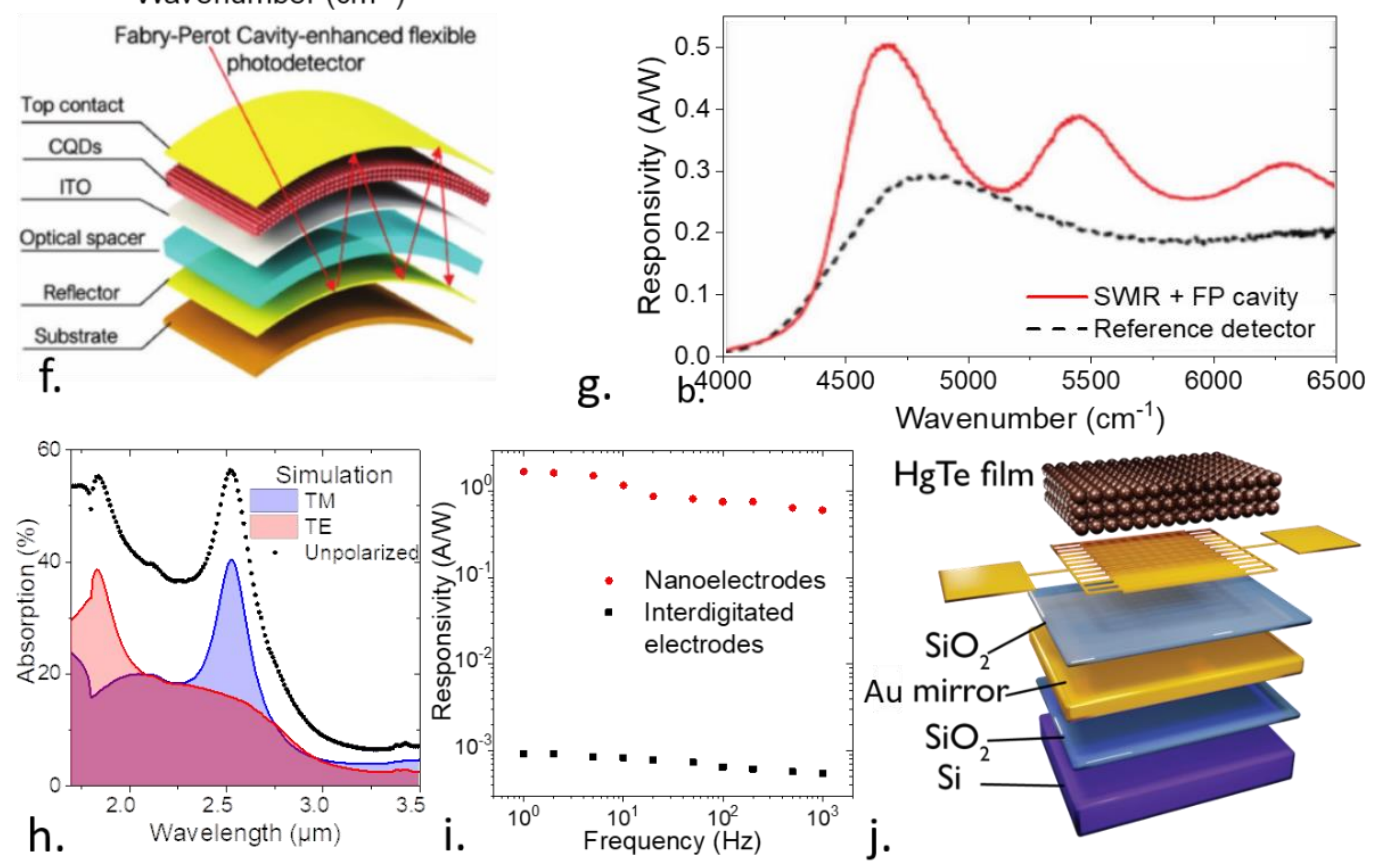

Figure 43 a. HgTe QD/ZnO heterojunction photodiode with Au nanorods embedded within the $\mathrm{ZnO}$ layer. b. Extinction increments of $\mathrm{HgTe} Q \mathrm{QD} / \mathrm{ZnO}$ films with $\mathrm{ZnO}$-coated Au nanorods normalized by the extinction spectrum of the control sample. The normalized FDTD simulated extinction cross-section spectra with the longitudinal and transverse LSPRs of Au nanorods coated with $7.5 \mathrm{~nm} \mathrm{ZnO}$ in the HgTe QD layer are also shown. Parts a and b are adapted with permission from ref ${ }^{389}$ Copyright (2014), American Chemical Society. c. Measured spectral responsivity of HgTe CQD MWIR detectors with and without plasmonic disks. d. Illustration of the HgTe CQD midwave infrared detector with plasmonic disks and a $30 \mathrm{~nm}$ Au contact. e. Mapping of the light intensity enhancement ratio. The wavelength of incident light is $\approx 4 \mu \mathrm{m}$. Parts $c-e$ are adapted with permission from ref ${ }^{38}$ Copyright (2018), American Chemical Society. f. Illustration of Fabry-Perot cavity-enhanced HgTe CQD detectors. g. Spectral responsivity of the SWIR HgTe detector with and without the Fabry-Perot cavity. Parts $f$ and $g$ are reprinted with permission from ref ${ }^{304}$, (c) 2019 WILEY-VCH Verlag GmbH \& Co. KGaA, Weinheim. $h$. Simulated absorption spectra relative to the TE mode, TM mode and total absorption for a $\mathrm{Hg} T \mathrm{NC}$ based device with a $1.8 \mu \mathrm{m}$ period. $i$. Responsivity as a function of the light signal frequency for devices based on HgTe nanocrystals using interdigitated electrodes made of gold contact deposited on a $\mathrm{Si} / \mathrm{SiO}_{2}$ substrate and interdigitated electrodes on a $\mathrm{Au} / \mathrm{SiO}_{2}$ substrate designed to induce GMR with a $1.8 \mu \mathrm{m}$ period. $j$. Schematics of the cross-section of the conventional interdigitated electrodes 
deposited on a Si/SiO 2 substrate. Parts $h-j$ are adapted with permission from ref ${ }^{40}$ Copyright (2019), American Chemical Society.

\subsection{Multicolor detection}

Multicolor detectors can be used to combine the specificities of each spectral band to clarify the exact nature of a scene. Visible sensors have the key advantage of being broadly available and being low cost. Shortwave infrared is commonly used for active imaging or moisture detection. The midinfrared domain corresponds to thermal imaging, which can be used for temperature determination or to conduct fully passive imaging. The combination of detectors to design bi- or multicolor detectors has already been well established. ${ }^{392}$ This high level of functionality was only recently developed for NC arrays. The developments of such multicolor detectors can only be achieved owing to the progress of the single color material detectors discussed earlier.

The first category of multicolor sensors includes a combination of several single color sensors. The first bicolor sensor based on nanocrystals was proposed by Lhuillier et al. ${ }^{41}$ The device is a visible/SWIR detector; see the scheme of the device in Figure $44 a$. The SWIR detection part is made of HgTe NCs, while the visible part is made of CdSe/CdS 2D nanoplatelets. ${ }^{67,183}$ The films of the two materials are connected in parallel, while the switch from a SWIR to visible response is enabled by the gating of the CdSe/CdS NPLs. When no gate bias is applied, the HgTe film, due to its narrow bandgap nature, is more conductive, and the photoresponse is driven by this material: both visible and SWIR present photoconduction (see Figure 44b). When a gate bias is applied, the conductance of the NPL film increases and overcomes that of the HgTe film owing to which only a visible response is observed (see Figure 44c). Tang et al. ${ }^{45}$ proposed a three color IR sensor at $5 \mu \mathrm{m}, 6 \mu \mathrm{m}$ (MWIR), and $9.5 \mu \mathrm{m}$ (LWIR). Fabrication of the device was based on stamping. Each active material was selectively deposited on interdigitated electrodes; see Figure 44d and e. Cryer et $a l .{ }^{42}$ proposed a similar device based on 4 colors in the SWIR and MWIR. The selective deposition of the material was realized by combining spray coating and shadow masking on a flexible PET substrate.
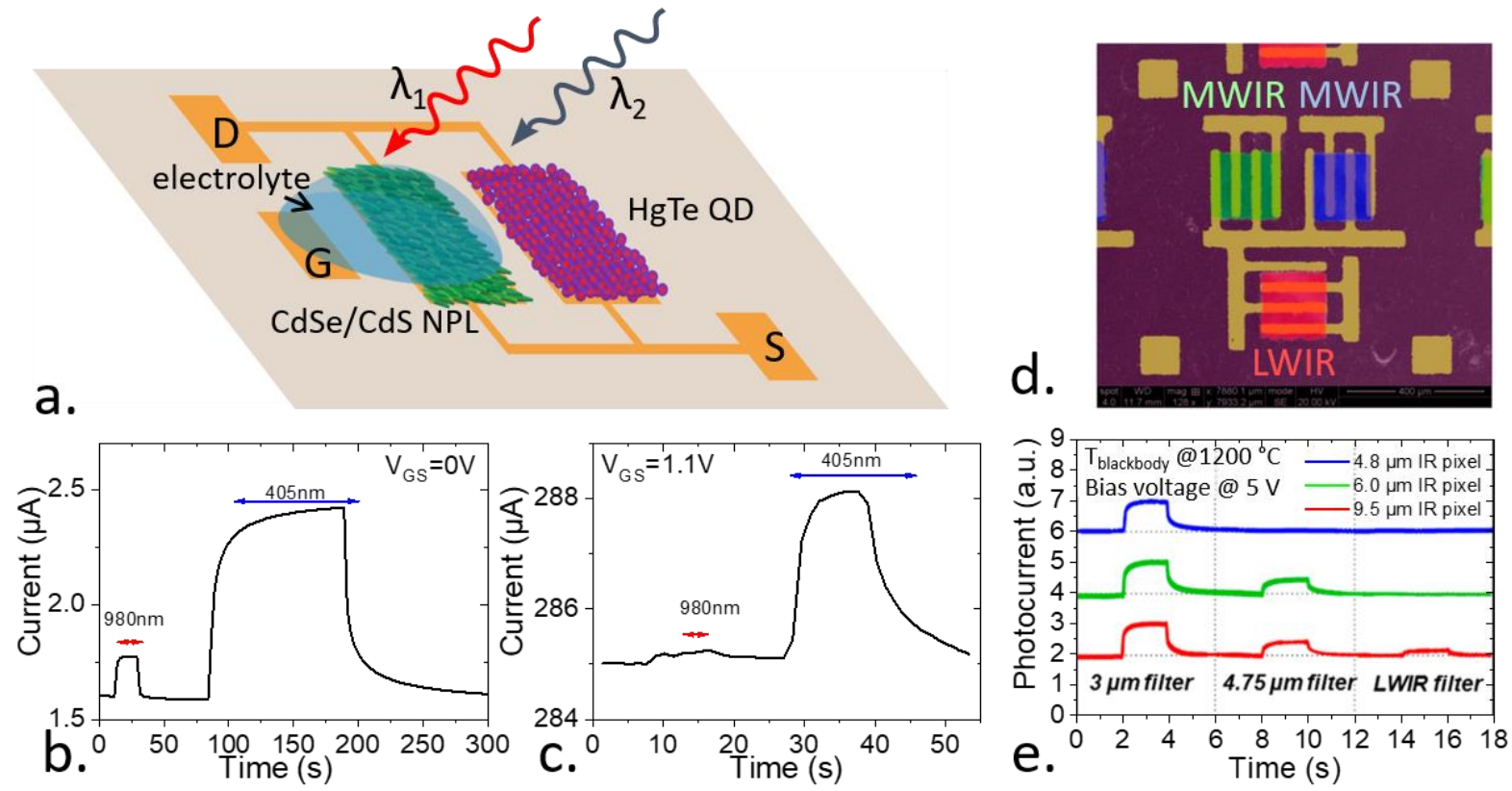

Figure 44 a. Scheme of a dual color device. b. (resp. c.) Current as a function of time under illumination at 405 and $980 \mathrm{~nm}$ at an applied gate bias $=0 \mathrm{~V}$ (resp. $1.1 \mathrm{~V}$ ). Parts a-c are adapted with permission from ref ${ }^{41}$ Copyright (2014), American Chemical Society d. Faux color SEM image of a multispectral photodetector. e. Photocurrent as a function of time of all 3 pixels under pulses of illumination from a blackbody with different filters. Parts $d$ and e are adapted with permission from ref ${ }^{45}$ Copyright (2016), American Chemical Society.

A second concept to design bi- and multicolor NC based sensors is to design a single device in which the spectral response can typically be tuned by applying a bias. Tang et al. proposed a SWIR/MWIR photodiode ${ }^{46}$ consisting of a stack of an MWIR ${ }^{303}$ diode and a SWIR ${ }^{304}$ diode; see Figure $45 \mathrm{a}$. The bias was used to switch the main contribution of the photoresponse from the SWIR to the MWIR diode; see Figure 45b. By coupling this diode to a mechanical scanning method, the authors realized bicolor imaging (see Figure $45 c-e)$. The same group recently proposed a visible/SWIR bicolor detector. ${ }^{43}$ The visible response was obtained from 
a Si wafer, while the SWIR response was collected from a HgTe NC film. A graphene layer was used as the intermediate transparent electrode. This device is especially promising because it can be easily transposed to a complementary metal oxide semiconductor (CMOS) readout circuit. Finally, a SWIR/MWIR sensor was proposed by Livache et al. by coupling the interband and intraband absorption from $\mathrm{HgTe}$ and $\mathrm{HgSe}$, respectively; see Figure $45 \mathrm{~h}$ and i. ${ }^{47}$

Although the existing devices allow the switching from one spectral range to another, Tang et al. proposed a hyperspectral sensor. In this case, the multicolor detection was no longer driven by the material but by the optical filtering of the photoresponse. To build such a device, a HgTe layer is coupled to an array of Bragg mirrors; see Figure 45f. This resonator allowed extremely narrow band selection (approximately $30 \mathrm{~cm}^{-1}$ ), enabling the selection of 64 bands in the SWIR and MWIR (see Figure $45 \mathrm{~g}$ ).
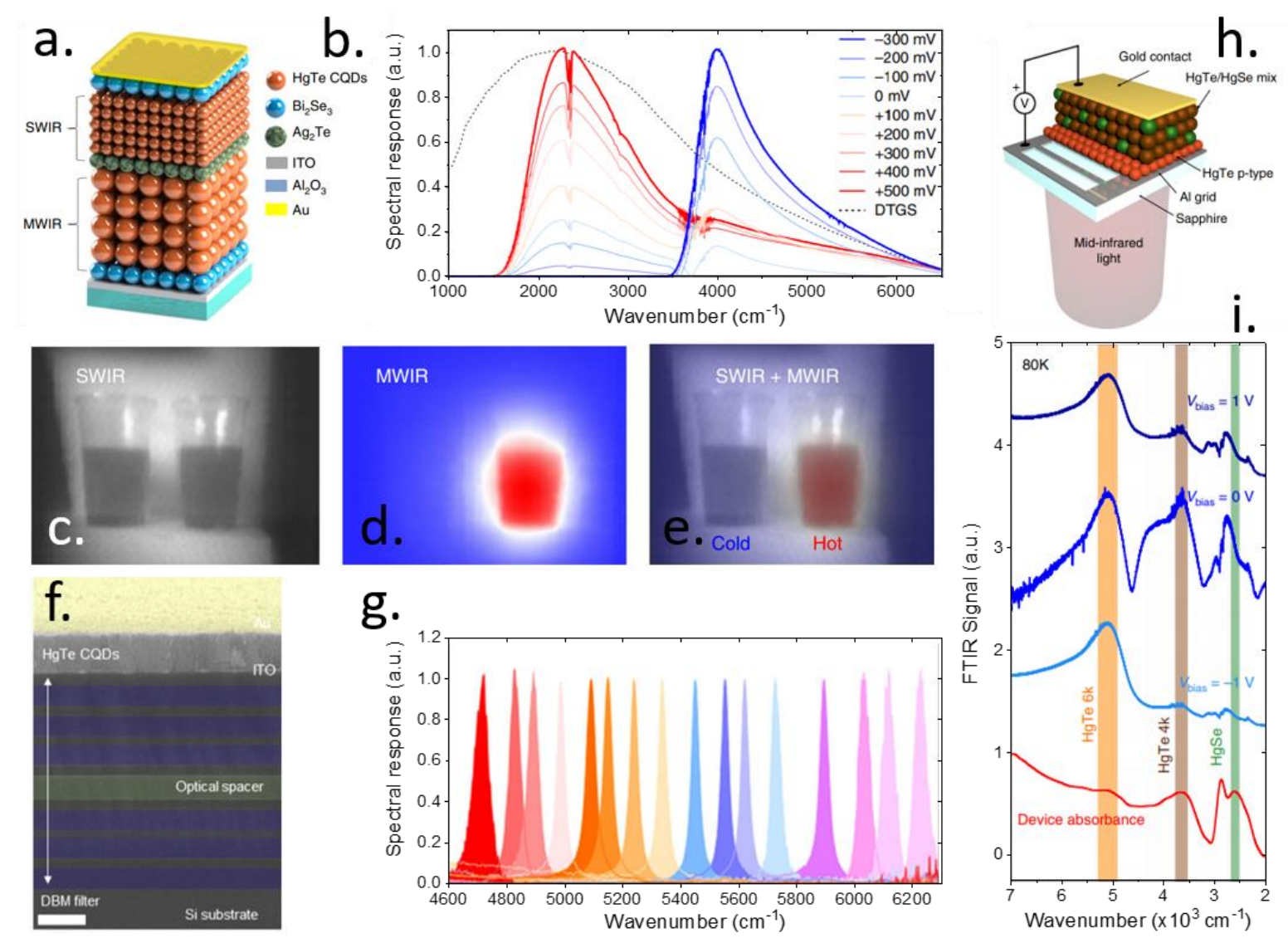

Wavenumber $\left(\times 10^{3} \mathrm{~cm}^{-1}\right)$

Figure 45 a. Illustration of the structure of a dual band CQD imaging device. A bias voltage is applied between indium tin oxide (ITO) and the Au contact (grounded). b. Spectral response of the dual band detector given in part a. under different bias voltages from positive $(+500 \mathrm{mV})$ to negative $(-300 \mathrm{mV})$ voltages at $85 \mathrm{~K}$. The dashed black line is the response of the internal deuterated triglycine sulfate (DTGS) detector in the Fourier transform infrared (FTIR) spectrometer, which reflects the spectral irradiance of the internal FTIR blackbody. The threshold bias voltages for the MWIR photodiode dominated and SWIR photodiode dominated photocurrents were $+300 \mathrm{mV}$ and $-200 \mathrm{mV}$, respectively. SWIR (c.), MWIR (d.) and merged (e.) dual band images of hot and cold water. The SWIR and MWIR images are obtained at room temperature and $85 \mathrm{~K}$, respectively. Parts a-e are reprinted with permission from Nature, Nature Photonics, ref ${ }^{46}$, [COPYRIGHT] (2019)f. False color cross-sectional scanning electron microscopy image of the CQD hyperspectral detector. The overlapping region between ITO and Au defines the active sensing area. Scale bar: $500 \mathrm{~nm}$. g. Measured spectral responsivity of fabricated CQD hyperspectral detectors. Parts $f$ and $g$ are reprinted with permission from ref ${ }^{44}$, (C) 2019 WILEY-VCH Verlag GmbH \& Co. KGaA, Weinheim. h. Scheme of the HgTe:HgSe diode. Illumination is provided from the backside through a sapphire substrate and patterned aluminum electrode, allowing $70 \%$ of light transmission in the MWIR. i. Photocurrent spectra and device absorbance measured at $80 \mathrm{~K}$ for several diode biases. The spectral contributions (maximum absorbance) of $\mathrm{HgSe}$ (green), $\mathrm{HgTe} 4 \mathrm{k}$ (bandgap at $4000 \mathrm{~cm}^{-1}$, brown) and $\mathrm{HgTe} 6 \mathrm{k}$ (bandgap at 6000 $\mathrm{cm}^{-1}$, orange) are highlighted. Figures $h$ and $i$ are adapted from ref ${ }^{47}$. 


\subsection{Integration and imaging}

Infrared nanocrystals have been discussed extensively and emerged as a possible alternative for low cost infrared imaging. The demonstration of imaging with infrared nanocrystals is relatively recent and enabled by their higher material maturity. Device integration, as opposed to single particle measurements, requires concentrated syntheses with a production of at least $100 \mathrm{mg}(1$ $\mathrm{g}$ is preferable even at the research level). ${ }^{29,96}$ Moreover, the material must present a certain degree of stability (toward air and aggregation). The demonstration of single pixel devices is a second mandatory step that allows one to understand the potential of the material compared to the existing alternatives. Alternatives to III-V and II-VI semiconductors for IR detection have been extensively studied, including colloidal materials; however, only a few recent reports presenting credible competitors with historical materials have been published. Infrared absorption is not sufficient to achieve high performance devices. Imaging devices must combine large photon-to-current conversion, fast response time (compatible with video frame rate), low noise, and material processability to obtain high quality thick films that strongly and homogeneously absorb over at least $1 \mathrm{~cm}^{2}$. All these reasons have delayed the demonstration of NC-based imaging setups. ${ }^{86} \mathrm{HgX}$, particularly $\mathrm{HgTe}$, has reached this level of maturity and emerged as a versatile platform for infrared optoelectronics. Consequently, the coupling of HgTe films to CMOS electronics has become a recent trend in the field, stimulated by the demonstration of focal plane arrays.

The design of nanocrystal-based devices relies on nonfunctionalized substrates, such as glass or silicon wafers. The substrate is mostly used as a mechanical sample holder, with possible constraints regarding the transparency. FETs are among the few early devices in which electrical functionalities from the substrate were expected. As the material maturity increased, coupling to Si electronics became possible. In the field of solar cells, it is unlikely that NCs can substitute for Si technology. The trend is generally to design tandem cells in which NCs lead to additional absorption in the silicon layer. ${ }^{393} \mathrm{~A}$ similar concept was proposed by Guyot Sionnest and coworkers to design a bicolor detector in which a Si layer absorbing in the visible and near IR was coupled to HgTe NCs to achieve SWIR photoconduction. ${ }^{43}$ Rogach and coworkers also proposed a device architecture that extended the advantage to Si coupling. The authors proposed the integration of HgTe NCs as IR absorbers in a silicon waveguide. ${ }^{388}$ This waveguide enhanced the incident electromagnetic field and thus obtained high absorption in a small volume. A reduced volume was expected to reduce the dark current of the device. A second benefit from this volume reduction is the reduction in the amount of $\mathrm{HgTe} \mathrm{NCs}$, which is a promising approach to obtain greener devices. By using silicon-based resonators, the advancements of Si photonics can be exploited, particularly for the design of high quality factor resonators.
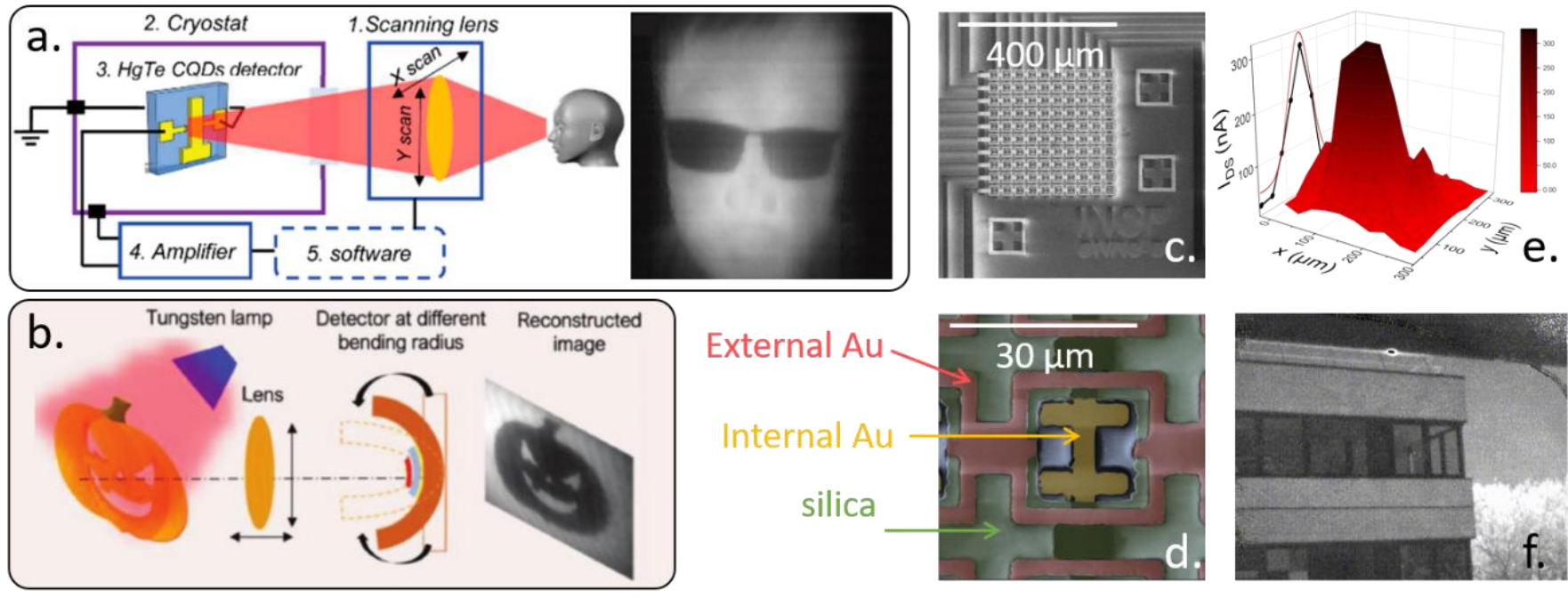

Figure 46 a. (left) Illustration of the HgTe CQD detector thermal imaging system. (right) Thermal images captured by HgTe CQD MWIR detectors. The detector was operated at $90 \mathrm{~K}$. Part a is adapted with permission from ref ${ }^{38}$ Copyright (2018), American Chemical Society. b. Simplified setup of infrared imaging with the flexible detector. A tungsten lamp is used to provide active infrared illumination. Part $b$ is reprinted with permission from ref ${ }^{304}$ (C) 2019 WILEY-VCH Verlag GmbH \& Co. KGaA, Weinheim. c. SEM image of the 100 pixel matrix (magnified 200x). d. Zoomed SEM image of a pixel with faux colors corresponding to different layers: yellow, green, and red represent the internal gold contact (columns), insulating silica and external gold contact (lines) (magnified 3000x), respectively. Blue is the glass substrate. e. Intensity of the 97 working pixels with the laser spot focused on the matrix. The Gaussian shape of the beam has a full width at half maximum (FWMH) of $75 \mu \mathrm{m}$. Parts $c-e$ are reprinted with permission from ref ${ }^{146}$. (c) 2019 WILEY-VCH Verlag GmbH \& Co. KGaA, Weinheim. f. Image from a HgTe nanocrystal film connected to CMOS ROIC, with no other lighting except for natural light (i.e., 

spectrum. Part $f$. is adapted from ref ${ }^{50}$.

Before discussing the design of multipixel sensors and focal plane arrays, it is worth noting that imaging can be obtained from a single pixel or a line of pixels coupled to a scanning system. This method has been extensively investigated by Tang et al. ${ }^{38,304}$, as illustrated in Figure $46 a$ and b, with application to dual color detection, ${ }^{46}$ as shown in Figure $45 c-e$. Generally, the device operates under cryogenic conditions (i.e., inside a cryostat), which makes displacement of the detector difficult. Therefore, the authors scanned the position of the optical lens to reconstruct an image. This method is slow due to mechanical displacement and is more suitable for picture acquisition than live imaging. Another issue is the presence of aberrations from the lens. The authors overcame this difficulty by designing a device with enhanced light-matter coupling on a flexible substrate to obtain an eyelike sensor. ${ }^{304}$

Currently, the design of FPAs is a topic that attracts the most interest relative to the coupling of HgX layers to Si-based electronics. This step is critical to realize the design of low cost sensors. The cost of an IR sensor is split over three main contributions: (i) growth of the active layer (which can be significant for liquid phase epitaxy of HgCdTe epitaxially matched on a CdZnTe substrate); (ii) hybridization, in which the active layer is coupled to the readout circuit (ROIC); and (iii) the packaging (including the cooling system if needed and optics, among other aspects). To obtain a notable cost reduction for the final FPA, it is mandatory to optimize all the aforementioned aspects in addition to the simple cost difference of the colloidal and epitaxial growth. In this scenario, it is unlikely for low cost sensors to have a cryogenic operating temperature, the cost of cryocoolers remaining prohibitive. If near room temperature is required, then it would be more promising to employ SWIR materials compared to MWIR materials because their larger bandgap corresponds to a reasonably low thermally activated carrier density. NCs represent the most promising materials to reduce the hybridization cost. At present, the active layer of III-V or II-VI semiconductors is coupled to the CMOS readout circuit through indium bumps (see Figure 47a). This process presents two severe drawbacks. First, this process is an expensive step with a limited yield, which further increases the cost. Second, this process is challenging for small pixel pitches. Existing cameras have a $15 \mu \mathrm{m}$ pitch, and the trend is to reduce this size by approximately $10 \mu \mathrm{m} .{ }^{394}$ This value is considerably larger than that achieved for CMOS visible sensors, for which pixel pitches of $2 \mu \mathrm{m}$ are commonly used. Thus, the quality of the SWIR image is not diffraction limited. Using NCs, it is possible to directly spread the NC solution onto the surface of the ROIC, which eases the fabrication and provides opportunities for smaller pixel design.

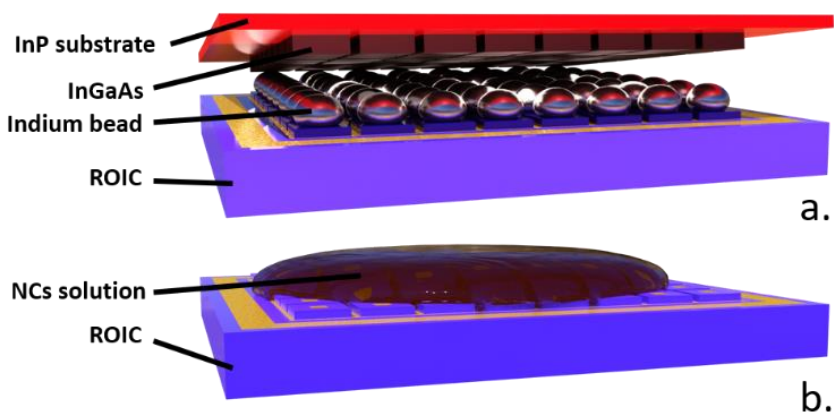

Figure 47 a. Scheme of an InGaAs layer grown on its InP substrate and coupled to a readout circuit (ROIC) through indium bumps. b. Scheme of an NC thin film deposited on a readout circuit (ROIC).

Ironically, the functionalization of ROICs with HgTe NCs was first reported in the MWIR range. ${ }^{51,52} \mathrm{HgTe}$ NCs with a cutoff of approximately $5 \mu \mathrm{m}$ were spin coated on top of the ROIC (see Figure 47b), and this step was followed by a solid state ligand exchange procedure. Images with clear evidence of thermal contrast and NETD below $100 \mathrm{mK}$ were obtained. In the SWIR, the first multipixel devices were based on small arrays of 10x10 pixels, as shown in Figure $46 \mathrm{c}$ and d. This matrix size was extremely small to achieve high quality images but could allow the function as an infrared beam profiler, ${ }^{146}$ as shown in Figure $46 e$. This application is particularly well suited for nanocrystal-based FPAs, for which imaging scenes presenting a large number of photons occur frequently since the amount of dark current is higher than that in the case of InGaAs detectors. Later, Chu et al. deposited an ink of HgTe NCs on a VGA ROIC and used this FPA as an infrared beam profiler, albeit with a significantly improved resolution. ${ }^{49}$ Finally, by enhancing the quality of ink deposition, Greboval et al. demonstrated passive imaging with these VGA FPAs; see the image in Figure 46f. No memory effect was observed in these devices, and framerates compatible with live imaging could be achieved. ${ }^{50}$ 


\section{CONCLUSIONS}

Over the past few decades, significant progress has been achieved for HgX NCs and their integration in devices. For material growth, a key aspect relates to size control, which has allowed the absorption of $\mathrm{HgX}$ materials to increase from $3.7 \mu \mathrm{m}^{27}$ in 2006 to $60 \mu \mathrm{m}$ at present. ${ }^{30}$ The other key development from NC growth is the discovery of self-doped NCs presenting intraband absorption features. ${ }^{33,65,66}$ The use of such transitions has been facilitated by the development of optimal experimental methods to clarify the doping mechanism and strategies to tune the corresponding magnitude. At this stage, intraband devices remain less effective than their interband competitors. However, intraband NCs are particularly interesting for the design of new devices operating at longer wavelengths and for the development of low toxicity alternatives.

The global knowledge and understanding of the material have also been considerably improved. Ten years ago, the modeling of $\mathrm{HgX}$ compounds was more or less limited to an effective mass approximation to determine the energy vs. size dependence. Significant theoretical efforts based on multiband k.p, ${ }^{62}$ tight binding, ${ }^{28,61,216}$ and DFT calculations have been dedicated to the understanding of properties such as the conduction band fine structure, temperature dependence of the spectroscopic properties, or the emergence of 4 level systems for stimulated emission.

In terms of light emissions, which represented the most mature aspect $10 \mathrm{y}$ ago, the initial trend was to extend PL emissions toward redder wavelengths. A value of $5 \mu \mathrm{m}$ at room temperature appears to be the current limit owing to the low PL efficiency. ${ }^{95}$ PL magnitude reduction is intrinsic to infrared wavelengths, and the room for enhancement (e.g., with heterostructures) is typically one order of magnitude. Stimulated emission ${ }^{22}$ and electroluminescence ${ }^{23}$ have been demonstrated over recent years, although a laser has not been developed yet.

On the detection side, tremendous progress has been made, including mid-IR photoconduction, high mobility films, near unity absorption devices, ${ }^{40}$ multicolor detection, demonstration of stability up to a three month time scale, and demonstration of focal plane arrays in the SWIR and MWIR ranges. It was believed that from the detection perspective, interest in HgTe would be driven by thermal imaging in the MWIR. This early belief was actually driven by the material and academic motivations to use HgTe NCs in a range of wavelengths beyond those achieved by lead chalcogenide materials. In the MWIR, the applications mostly relate to defense and astronomy, for which cost is not a major constraint and extremely high performance and reliability are desired. The SWIR domain is presently exhibiting an interest in low cost detection. This range of wavelengths suffers from the vicinity of the visible range, for which extremely high performance imaging is available with inexpensive applications. A VGA format Si-based FPA in the visible region based on CMOS technology costs less than $\$ 1$ and achieves excellent performances up to $1 \mu \mathrm{m}$, while the InGaAs equivalent operating at up to $1.7 \mu \mathrm{m}$ is considerably more expensive (more than $\$ 100)$. This notable difference in the cost for a marginal shift of the cutoff wavelength is typically not well understood by customers outside the detection community. Infrared NC-based FPAs exhibit considerably lower performances than those of FPAs based on InGaAs; consequently, applications for which a dark current is not a limitation should be targeted. A clear application is infrared beam profiling. Because of the large number of photons available in this application, this scenario is compatible with moderate responsivity devices. Active imaging is another application in which a dark current is not a limiting factor since the scene is illuminated. Various applications relative to industrial vision fall in this type of application, including waste sorting (SWIR is used to distinguish metal from plastic or paper), moisture detection (water strongly absorbs in the SWIR range), and food damage detection (broken eggs, fruit damage, etc.). Detection for LIDAR may also be considered owing to the short response time of HgTe devices, although this aspect is expected to be challenging because of the reduced photon flux. 


\section{FUTURE DEVELOPMENTS}

\subsection{Need for better materials}

\subsubsection{Stability}

While the performance of $\mathrm{HgX} \mathrm{NC}$-based devices has been enhanced, transfer toward the mass market requires a demonstration of the viability of long term operations. Stability is the main issue due to the corresponding performance enhancement. If this issue can be effectively addressed, then $\mathrm{HgX} \mathrm{NCs}$ will enter a new phase of development. The objective is for the material to be operated without irreversible damage up to $60^{\circ} \mathrm{C}$ while being processable with limited damage up to $150{ }^{\circ} \mathrm{C}$. Hybrid passivation combining halides and organic ligands can enhance film stability, ${ }^{36}$ and multilayer encapsulation based on water and oxygen repelling layers can ensure room temperature stability. ${ }^{302}$ These objectives have not been attained yet. Low temperature damage prevents the use of various encapsulation methods. Material developments must be facilitated to enable such encapsulations. The solutions will likely require the development of new core-shell growths, making large scale syntheses more complicated. However, this aspect can be realized since display applications have demonstrated that complex core-shell nanoparticles can be grown at a large scale. However, even if the scale up of $\mathrm{HgX} \mathrm{NC}$ can benefit from the developments of CdSe and InP, the change in material will require a certain update of the procedure to account for the specificity of the $\mathrm{HgX}$ compound, such as low $\mathrm{T}$ growth.

\subsubsection{Toxicity}

Although this review is focused on $\mathrm{HgX}$ materials, the quest to achieve low toxicity must be considered. The current situation regarding toxicity is not as complex as that for solar cells. When CdTe-based solar cells were developed for third generation solar cells, in addition to the undeniable toxicity of $\mathrm{Cd}$, the supply of a large amount of Te was under debate. There is a fundamental size difference between IR sensors and solar cell devices. A VGA format focal plane array is a $1 \mathrm{~cm}^{2}$ square chip, while a solar cell requires $\mathrm{m}^{2}$ devices. Livache et al. estimated that introducing a $\mathrm{HgX} \mathrm{NC}$-based focal plane array in every car sold in Europe (20 million unit/year) will "only" require a few tens of kg of HgX NCs. ${ }^{395}$

Another focus is on alternatives materials. The current industry dedicated to IR sensing is already used to managing II-VI compounds. While this industry has demonstrated interest in the NC form, the presence of Hg still retards the industrial transfer of $\mathrm{HgX} \mathrm{NCs}$ despite their reported promising performance. A heavy metal free alternative with a similar performance to that achievable using HgTe may be a breakthrough technique. Various materials have been developed to achieve IR photoconduction, including graphene, black phosphorus, ${ }^{396-400}$ platinum $^{401,402}$ and paladium ${ }^{403,404}$ dichalcogenides, silver chalcogenides, ${ }^{77-79,405,406}$ and doped oxides (ITOs). ${ }^{76}$ For example, $\mathrm{Ag}_{2} \mathrm{Se}$ can easily be grown in NC form. The infrared spectrum is surprisingly similar to that of $\mathrm{HgSe}$. In addition, $\mathrm{Ag}_{2} \mathrm{Se}$ is a chalcogenide $\mathrm{NC}$, and it is natural to assume that the knowledge transfer from $\mathrm{PbX}, \mathrm{CdS}$, and $\mathrm{HgX}$ materials can ease the integration of this material into devices. Recent demonstrations have been rather disappointing. In particular, materials exhibit low electrochemical stability, and the silver atoms undergo changes in redox states while obtaining IV curves $^{79}$. The performance for IR photodetection is several orders of magnitude lower than that measured for HgX NCs. Similarly, the potential of ITO NCs has been evaluated. The ITO spectrum is widely tunable in the midinfrared region through the doping magnitude. ITO is a plasmonic material, and a higher cross-section was anticipated compared to that for intraband absorption. However, this benefit is more than balanced by the extremely short lifetime of plasmons ( $\mathrm{a}$ few fs). Consequently, the infrared photoresponse obtained in devices made from this material is not due to photoconduction but to bolometric effects. ${ }^{76}$

One of the challenges, while trying to develop new materials for IR sensing is to move beyond the photoconduction proof of concept. The latter aspect corresponds to a technological readiness level of 1, while a level of 3 or 4 is required for the industry to take over. In addition to photoconduction, noise and stability also must be evaluated. The next level of maturity addresses the technology transfer from a single pixel to imaging setups. This effort is generally performed in parallel to on-chip integration. To date, only PbS and HgTe NCs are sufficiently mature to be integrated into devices.

While the quest for achieving low toxicity materials has been moderately successful for the design of IR sensors, we can try to develop "green" HgX NCs. In particular, all the environments of HgX NCs can be made greener. One step in this direction has been taken with the report of highly concentrated syntheses. ${ }^{96}$ The authors dramatically reduced solvent and ligand consumption. $\mathrm{A}$ similar effort for the ligand exchange step required to make the film conductive is also required. This phenomenon may be achieved through direct synthesis of the NCs into polar solvents, as proposed by Kershaw et al. ${ }^{92}$. Film-making techniques should also be revised: the current trend is to use spin coating deposition onto a substrate, electrodes or readout circuit. This technique is responsible for a considerable loss of NCs, which is currently the major source of waste, generating high costs and excessive consumption of heavy metals. Better deposition techniques must be developed. Inkjet printing ${ }^{407,408}$ or stamping $^{45}$ may be able to be used, as only the needed amount of material must be deposited. In addition, this aspect may enable the deposition of NCs at 
the wafer scale rather than the chip scale. While NC deposition by these methods has been reported, one must not neglect the time required to transfer these skills to $\mathrm{HgX} \mathrm{NCs.}$

\subsection{Need for deeper material understanding}

A clear trend for both light emission and detection is the development of light-matter coupling. In other words, the spectral response of a detector is no longer restricted to the intrinsic optical properties of the material. The introduction of photonic structures (plasmonic resonators, distributed feedback mirrors ${ }^{44}$ ) has enabled the introduction of new degrees of freedom for the design of spectral responses, PL efficiency enhancement, ${ }^{21}$ or light focusing. ${ }^{38,40}$ This direction will undoubtedly be expanded in the coming years. While the design of the light-matter coupling is strongly correlated to electromagnetic simulation tools, the amount of work dedicated to the measurement of the optical properties of HgX NCs remains comparatively low. ${ }^{114,228}$ Deeper and more systematic studies on the complex optical index need to be conducted to serve as inputs for simulations. One reason for this lack of data is the low availability of ellipsometers operating at wavelengths above $1.7 \mu \mathrm{m} .{ }^{409,410}$ Significant improvements have been achieved in terms of the film quality, ${ }^{146,303}$ particularly in terms of film roughness, and consequently, no fundamental limitation is expected to prevent such measurements in the coming years.

The determination of the band alignment of $\mathrm{HgX}$ materials with other materials or transport layers is critical for the growth of colloidal heterostructures and the design of photodiodes. To date, $\mathrm{HgX} \mathrm{NC}$-based photodiode design does not have a rational basis and is dictated by empirical laws. Future designs must account for the specificity of narrow bandgap materials and, consequently, must be increasingly inspired by solar cells, for which the required bandgap (and the associated band alignment) is two to three times larger. For this aspect, electrochemistry and photoemission must be more systematically used to clarify the band alignment as a function of the size and surface chemistry. It is likely that the interaction of the absorbing layer with electron and hole transport layers can help facilitate the design process.

\subsection{Need for better devices}

As mentioned previously, IR sensing based (mostly) on HgTe NCs has seen dramatically increased performances over the last few years, and the performances in SWIR are similar to those of the existing commercial devices. ${ }^{48}$ Although there remains room for device improvements, a breakthrough could be the realization of room temperature operation for MWIR detectors. From our perspective, the main technological challenge will be integration. None of the existing studies have reported on the integration of high performing new concepts (use of gates, plasmonic resonators) developed for single-pixel devices for FPAs. For instance, most resonators designed to achieve strongly absorbing films are not compatible with the pitch size of an FPA. In addition, in laboratory devices, illumination can be performed from both sides of the device; however, the presence of the readout circuit renders backside illumination impossible. These aspects must be considered by examining the interface between academic laboratories and industrial companies. Considering that the development cost of a dedicated ROIC is typically a million dollars, the cost required might also be significantly higher. 


\section{ABBREVIATIONS}

BLIP: Background Limited Infrared Photodetection

CdX: Cadmium Chalcogenides

CQD: Colloidal Quantum Dots

c-ALD: Colloidal Atomic Layer Deposition

DDT: Dodecanethiol

DMF: Dimethylformamide

DMSO: Dimethyl sulfoxide

EDT: Ethanedithiol

EDX: Energy Dispersive X-Ray Spectroscopy

EL: Electroluminescence

FET: Field Effect Transistor

FPA: Focal Plane Array

HgX: Mercury Chalcogenides

LWIR: Longwave Infrared

MEG: Multi-Exciton Generation

MWIR: Midwave Infrared

NC: Nanocrystal

NIR: Near Infrared

OLA : Oleylamine

PL: Photoluminescence

QCL: Quantum Cascade Laser

QD: Quantum Dots

ROIC: Readout Integrated Circuit

SEM: Scanning Electron Microscopy

SWIR: Short Wave InfraRed

TEM: Transmission Electron Microscopy

XPS (UPS): X-Ray (UV) Photoemission Spectroscopy

\section{ACKNOWLEDGMENTS}

The project is supported by the ERC starting grants blackQD (grant $n^{\circ} 756225$ ) and Ne2Dem (grant $n^{\circ}$ 853049). We acknowledge the use of the clean room facilities at the "Centrale de Proximite Paris-Centre". This work has been supported by Region lle-deFrance in the framework of DIM Nano-K (grant dopQD). This work was supported by the French state funds managed by the ANR within the Investissements d'Avenir programme under reference ANR-11-IDEX-0004-02 within the framework of the Cluster of Excellence MATISSE and by the grants IPER-Nano2 (ANR-18CE30-0023-01), Copin (ANR-19-CE24-0022), Frontal (ANR-19-CE090017), Graskop (ANR-19-CE09-0026) and NITQuantum. 


\section{BIOGRAPHIES}

Charlie Gréboval received his Advanced Master of Science and Engineering degree in 2018 from École Supérieure de Physique et de Chimie Industrielles de Paris (ESPCI Paris). He later joined the Parisian Institute of Nanoscience (INSP), Sorbonne Université, Paris, as a PhD candidate examining the transport and optical properties in narrow bandgap nanocrystals.

Audrey Chu received her bachelor's degree from École Supérieur de Physique et de Chimie Industrielle de la ville de Paris. After an internship in Sandrine Itthuria's group, she received a master's degree in material science and nanotechnology from Sorbonne University. She later joined the group of Emmanuel Lhuiller in 2018 to conduct her PhD. Her primary research interests include the design and study of light-matter coupled devices to enhance the photodetection properties of NC films.

Nicolas Goubet was born in Cherbourg, France, in 1982. He received a PhD in 2016 from the University Pierre and Marie Curie. He has fifteen years of experience in metal nanocrystal synthesis. He worked as a postdoctoral researcher with Emmanuel Lhuillier's group, developing new mercury chalcogenide synthesis techniques. He is currently an engineer assistant at the MONARIS laboratory at Sorbonne University.

Clément Livache received his Chimie ParisTech - ENSCP engineer diploma in 2016. He also holds a master's degree in materials sciences from Sorbonne Universite. He joined both Benoit Dubertret's group at ESPCI and Emmanuel Lhuillier's group at Sorbonne Universite in 2016 for a joint PhD. In this tenure, he was involved with device fabrication and characterization, with a focus on carrier dynamics and the exploitation of intraband transitions. He obtained a PhD degree in 2019 and is presently working as a postdoctoral research fellow at the Los Alamos National Laboratory in the team of Victor Klimov.

Sandrine Ithurria is an ESPCl engineer and holds a master's degree in chemistry from Sorbonne Université. She was a PHD student with Benoit Dubertret at ESPCl, where she realized the first growth of 2D zinc blende nanoplatelets. She later joined Talapin's group at the University of Chicago to study nanocrystal surface chemistry and develop a new method for low temperature shell growth. Since 2012, she has been an associate professor at ESPCI, with a research focus on 2D colloidal materials. In 2019, she was awarded an ERC starting grant.

Emmanuel Lhuillier is an ESPCl engineer and holds a master's degree in condensed matter from Sorbonne Université. To attain his PhD, he joined ONERA under the supervision of Emmanuel Ronsencher, where he examined the transport in a quantum well structure to be used as an infrared sensor. In the group of Philippe Guyot-Sionnest at the University of Chicago, he investigated the photoconduction properties of HgTe nanocrystals. He later joined the group of Benoit Dubertret at ESPCl, focusing on the optoelectronics of the colloidal quantum well. In 2015, he joined CNRS at Sorbonne Universite at the nanoscience institute of Paris, where his group studies the optoelectronics of confined nanomaterials, specifically, infrared nanocrystals. 


\section{REFERENCES}

(1) Böberl, M.; Kovalenko, M. V.; Gamerith, S.; List, E. J. W.; Heiss, W. Inkjet-Printed Nanocrystal Photodetectors Operating up to $3 \mu \mathrm{m}$ Wavelengths. Adv. Mater. 2007, 19, 3574-3578.

(2) Seong, H.; Cho, K.; Kim, S. Photocurrent Characteristics of Solution-Processed HgTe Nanoparticle Thin Films under the Illumination of $1.3 \mu \mathrm{m}$ Wavelength Light. Semicond. Sci. Technol. 2008, 23, 075011.

(3) Keuleyan, S.; Lhuillier, E.; Brajuskovic, V.; Guyot-Sionnest, P. Mid-Infrared HgTe Colloidal Quantum Dot Photodetectors. Nat. Photonics 2011, 5, 489-493.

(4) Kovalenko, M. V.; Scheele, M.; Talapin, D. V. Colloidal Nanocrystals with Molecular Metal Chalcogenide Surface Ligands. Science 2009, 324, 1417-1420.

(5) Lawson, W. D.; Nielsen, S.; Putley, E. H.; Young, A. S. Preparation and Properties of HgTe and Mixed Crystals of HgTeCdTe. J. Phys. Chem. Sol. 1959, 9, 325-329.

(6) Murray, C. B.; Norris, D. J.; Bawendi, M. G. Synthesis and Characterization of Nearly Monodisperse CdE (E = Sulfur, Selenium, Tellurium) Semiconductor Nanocrystallites. J. Am. Chem. Soc. 1993, 115, 8706-8715.

(7) Peng, X.; Manna, L.; Yang, W.; Wickham, J.; Scher, E.; Kadavanich, A.; Alivisatos, A. P. Shape Control of CdSe Nanocrystals. Nature 2000, 404, 59-61.

(8) Ithurria, S.; Tessier, M. D.; Mahler, B.; Lobo, R. P. S. M.; Dubertret, B.; Efros, A. L. Colloidal Nanoplatelets with TwoDimensional Electronic Structure. Nat. Mater. 2011, 10, 936-941.

(9) Hines, M. A.; Guyot-Sionnest, P. Synthesis and Characterization of Strongly Luminescing ZnS-Capped CdSe Nanocrystals. J. Phys. Chem. 1996, 100, 468-471.

(10) Bourzac, K. Quantum Dots Go on Display. Nat. News 2013, 493, 283.

(11) Huang, Y.-M.; Singh, K. J.; Liu, A.-C.; Lin, C.-C.; Chen, Z.; Wang, K.; Lin, Y.; Liu, Z.; Wu, T.; Kuo, H.-C. Advances in QuantumDot-Based Displays. Nanomaterials 2020, 10, 1327.

(12) Leroy, S. Phosphors \& Quantum Dots 2015: LED Downconverters for Lighting \& Display, 2015.

(13) Leroy, S. Quantum Dots and Wide Color Gamut Display Technologies, 2017.

(14) Rogach, A. L.; Eychmüller, A.; Hickey, S. G.; Kershaw, S. V. Infrared-Emitting Colloidal Nanocrystals: Synthesis, Assembly, Spectroscopy, and Applications. Small 2007, 3, 536-557.

(15) Eychmüller, A.; Mews, A.; Weller, H. A Quantum Dot Quantum Well: CdS/HgS/CdS. Chem. Phys. Lett. 1993, $208,59-62$.

(16) Reiss, P.; Protière, M.; Li, L. Core/Shell Semiconductor Nanocrystals. Small 2009, 5, 154-168.

(17) Beberwyck, B. J.; Surendranath, Y.; Alivisatos, A. P. Cation Exchange: A Versatile Tool for Nanomaterials Synthesis. J. Phys. Chem. C 2013, 117, 19759-19770.

(18) Son, D. H.; Hughes, S. M.; Yin, Y.; Alivisatos, A. P. Cation Exchange Reactions in lonic Nanocrystals. Science 2004, 306, 1009-1012.

(19) De Trizio, L.; Manna, L. Forging Colloidal Nanostructures via Cation Exchange Reactions. Chem. Rev. 2016, 116, 1085210887.

(20) Rogach, A.; Kershaw, S. V; Burt, M.; Harrison, M. T.; Kornowski, A.; Eychmüller, A.; Weller, H. Colloidally Prepared HgTe Nanocrystals with Strong Room-Temperature Infrared Luminescence. Adv. Mater. 1999, 11, 552-555.

(21) Sergeev, A. A.; Pavlov, D. V.; Kuchmizhak, A. A.; Lapine, M. V.; Yiu, W. K.; Dong, Y.; Ke, N.; Juodkazis, S.; Zhao, N.; Kershaw, S. V.; et al. Tailoring Spontaneous Infrared Emission of HgTe Quantum Dots with Laser-Printed Plasmonic Arrays. Light: Sci. Appl. 2020, 9, 16.

(22) Geiregat, P.; Houtepen, A. J.; Sagar, L. K.; Infante, I.; Zapata, F.; Grigel, V.; Allan, G.; Delerue, C.; Van Thourhout, D.; Hens, Z. Continuous-Wave Infrared Optical Gain and Amplified Spontaneous Emission at Ultralow Threshold by Colloidal HgTe Quantum Dots. Nat. Mater. 2018, 17, 35-42.

(23) Qu, J.; Rastogi, P.; Gréboval, C.; Lagarde, D.; Chu, A.; Dabard, C.; Khalili, A.; Cruguel, H.; Robert, C.; Xu, X. Z.; et al. Electroluminescence from HgTe Nanocrystals and Its Use for Active Imaging. Nano Lett. 2020, 20, 6185-6190.

(24) Nag, A.; Kovalenko, M. V.; Lee, J.-S.; Liu, W.; Spokoyny, B.; Talapin, D. V. Metal-Free Inorganic Ligands for Colloidal Nanocrystals: $\mathrm{S}^{2-}, \mathrm{HS}^{-}, \mathrm{Se}^{2-}, \mathrm{HSe}^{-}, \mathrm{Te}^{2-}, \mathrm{HTe}^{-}, \mathrm{TeS}_{3}{ }^{2-}, \mathrm{OH}^{-}$, and $\mathrm{NH}^{2-}$ as Surface Ligands. J. Am. Chem. Soc. 2011, 133, 10612-10620.

(25) Fafarman, A. T.; Koh, W.; Diroll, B. T.; Kim, D. K.; Ko, D.-K.; Oh, S. J.; Ye, X.; Doan-Nguyen, V.; Crump, M. R.; Reifsnyder, D. C.; et al. Thiocyanate-Capped Nanocrystal Colloids: Vibrational Reporter of Surface Chemistry and Solution-Based Route to Enhanced Coupling in Nanocrystal Solids. J. Am. Chem. Soc. 2011, 133, 15753-15761.

(26) Tang, J.; Kemp, K. W.; Hoogland, S.; Jeong, K. S.; Liu, H.; Levina, L.; Furukawa, M.; Wang, X.; Debnath, R.; Cha, D.; et al. Colloidal-Quantum-Dot Photovoltaics Using Atomic-Ligand Passivation. Nat. Mater. 2011, 10, 765-771.

(27) Kovalenko, M. V.; Kaufmann, E.; Pachinger, D.; Roither, J.; Huber, M.; Stangl, J.; Hesser, G.; Schäffler, F.; Heiss, W. Colloidal HgTe Nanocrystals with Widely Tunable Narrow Band Gap Energies: From Telecommunications to Molecular Vibrations. J. Am. Chem. Soc. 2006, 128, 3516-3517.

(28) Keuleyan, S. E.; Guyot-Sionnest, P.; Delerue, C.; Allan, G. Mercury Telluride Colloidal Quantum Dots: Electronic Structure, Size-Dependent Spectra, and Photocurrent Detection up to $12 \mu \mathrm{m}$. ACS Nano 2014, 8, 8676-8682. 
Lhuillier, E.; Scarafagio, M.; Hease, P.; Nadal, B.; Aubin, H.; Xu, X. Z.; Lequeux, N.; Patriarche, G.; Ithurria, S.; Dubertret, B. Infrared Photodetection Based on Colloidal Quantum-Dot Films with High Mobility and Optical Absorption up to THz. Nano Lett. 2016, 16, 1282-1286.

(30) Goubet, N.; Jagtap, A.; Livache, C.; Martinez, B.; Portalès, H.; Xu, X. Z.; Lobo, R. P. S. M.; Dubertret, B.; Lhuillier, E. Terahertz HgTe Nanocrystals: Beyond Confinement. J. Am. Chem. Soc. 2018, 140, 5033-5036.

(46) Tang, X.; Ackerman, M. M.; Chen, M.; Guyot-Sionnest, P. Dual-Band Infrared Imaging Using Stacked Colloidal Quantum

Dot Photodiodes. Nat. Photonics 2019, 13, 277-282. Izquierdo, E.; Robin, A.; Keuleyan, S.; Lequeux, N.; Lhuillier, E.; Ithurria, S. Stro
Narrow Near-Infrared Emitters. J. Am. Chem. Soc. 2016, 138, 10496-10501.

Rogach, A. L.; Harrison, M. T.; Kershaw, S. V.; Kornowski, A.; Burt, M. G.; Eychmüller, A.; Weller, H. Colloidally Prepared CdHgTe and HgTe Quantum Dots with Strong Near-Infrared Luminescence. Phys. Stat. Solidi. B 2001, 224, 153-158.

Jeong, K. S.; Deng, Z.; Keuleyan, S.; Liu, H.; Guyot-Sionnest, P. Air-Stable n-Doped Colloidal HgS Quantum Dots. J. Phys. Chem. Lett. 2014, 5, 1139-1143.

Deng, Z.; Jeong, K. S.; Guyot-Sionnest, P. Colloidal Quantum Dots Intraband Photodetectors. ACS Nano 2014, 8, 1170711714.

Lhuillier, E.; Keuleyan, S.; Zolotavin, P.; Guyot-Sionnest, P. Mid-Infrared HgTe/As $2 \mathrm{~S}_{3}$ Field Effect Transistors and Photodetectors. Adv. Mater. 2013, 25, 137-141.

Chen, M.; Lan, X.; Tang, X.; Wang, Y.; Hudson, M. H.; Talapin, D. V.; Guyot-Sionnest, P. High Carrier Mobility in HgTe Quantum Dot Solids Improves Mid-IR Photodetectors. ACS Photonics 2019, 6, 2358-2365.

Yifat, Y.; Ackerman, M.; Guyot-Sionnest, P. Mid-IR Colloidal Quantum Dot Detectors Enhanced by Optical Nano-Antennas. Appl. Phys. Lett. 2017, 110, 041106.

Tang, X.; Ackerman, M. M.; Guyot-Sionnest, P. Thermal Imaging with Plasmon Resonance Enhanced HgTe Colloidal Quantum Dot Photovoltaic Devices. ACS Nano 2018, 12, 7362-7370.

Tang, X.; Wu, G. fu; Lai, K. W. C. Plasmon Resonance Enhanced Colloidal HgSe Quantum Dot Filterless Narrowband Photodetectors for Mid-Wave Infrared. J. Mater. Chem. C 2017, 5, 362-369.

Chu, A.; Gréboval, C.; Goubet, N.; Martinez, B.; Livache, C.; Qu, J.; Rastogi, P.; Bresciani, F. A.; Prado, Y.; Suffit, S.; et al. Near Unity Absorption in Nanocrystal Based Short Wave Infrared Photodetectors Using Guided Mode Resonators. ACS Photonics 2019, 6, 2553-2561.

Lhuillier, E.; Robin, A.; Ithurria, S.; Aubin, H.; Dubertret, B. Electrolyte-Gated Colloidal Nanoplatelets-Based Phototransistor and Its Use for Bicolor Detection. Nano Lett. 2014, 14, 2715-2719.

Cryer, M. E.; Halpert, J. E. $300 \mathrm{~nm}$ Spectral Resolution in the Mid-Infrared with Robust, High Responsivity Flexible Colloidal Quantum Dot Devices at Room Temperature. ACS Photonics 2018, 5, 3009-3015.

Tang, X.; Chen, M.; Kamath, A.; Ackerman, M. M.; Guyot-Sionnest, P. Colloidal Quantum-Dots/Graphene/Silicon DualChannel Detection of Visible Light and Short-Wave Infrared. ACS Photonics 2020, 7, 1117-1121.

Tang, X.; Ackerman, M. M.; Guyot-Sionnest, P. Acquisition of Hyperspectral Data with Colloidal Quantum Dots. Laser \& Photonics Rev. 2019, 13, 1900165.

Tang, X.; Tang, X.; Lai, K. W. C. Scalable Fabrication of Infrared Detectors with Multispectral Photoresponse Based on Patterned Colloidal Quantum Dot Films. ACS Photonics 2016, 3, 2396-2404. Colloidal Quantum Dot Infrared Photodetector and Its Use for Intraband Detection. Nat. Commun. 2019, 10, 2125.

Ackerman, M. M.; Chen, M.; Guyot-Sionnest, P. HgTe Colloidal Quantum Dot Photodiodes for Extended Short-Wave Infrared Detection. Appl. Phys. Lett. 2020, 116.

Chu, A.; Martinez, B.; Ferré, S.; Noguier, V.; Gréboval, C.; Livache, C.; Qu, J.; Prado, Y.; Casaretto, N.; Goubet, N.; et al. HgTe Nanocrystals for SWIR Detection and Their Integration up to the Focal Plane Array. ACS Appl. Mater. Interfaces 2019, 11, 33116-33123.

Greboval, C.; Ferre, S.; Noguier, V.; Chu, A.; Qu, J.; Chee, S.-S.; Vincent, G.; Lhuillier, E. Infrared Narrow Band Gap Nanocrystals: Recent Progresses Relative to Imaging and Active Detection. arXiv:2001.11554 [cond-mat, physics:physics] 2020, 1-12.

Buurma, C.; Pimpinella, R. E.; Ciani, A. J.; Feldman, J. S.; Grein, C. H.; Guyot-Sionnest, P. MWIR Imaging with Low Cost Colloidal Quantum Dot Films. In Optical Sensing, Imaging, and Photon Counting: Nanostructured Devices and Applications 2016; International Society for Optics and Photonics, 2016, 9933, 993303.

Ciani, A. J.; Pimpinella, R. E.; Grein, C. H.; Guyot-Sionnest, P. Colloidal Quantum Dots for Low-Cost MWIR Imaging. In Infrared Technology and Applications XLII; International Society for Optics and Photonics, 2016, 9819, 981919.

Rogalski, A.; Kopytko, M.; Martyniuk, P. Comparison of Performance Limits of HOT HgCdTe Photodiodes and Colloidal Quantum Dot Infrared Detectors. In Infrared Technology and Applications XLVI; International Society for Optics and Photonics, 2020, 11407,114070V.

Green, M.; Mirzai, H. Synthetic Routes to Mercury Chalcogenide Quantum Dots. J. Mater. Chem. C 2018, 6, 5097-5112. Kershaw, S. V.; Rogach, A. L. Infrared Emitting HgTe Quantum Dots and Their Waveguide and Optoelectronic Devices. Z.

Phys. Chem. 2015, 229, 23-64.
Lhuillier, E.; Keuleyan, S.; Liu, H.

Lhuillier, E.; Keuleyan, S.; Liu, H.; Guyot-Sionnest, P. Mid-IR Colloidal Nanocrystals. Chem. Mater. 2013, 25, 1272-1282. 
Lhuillier, E.; Guyot-Sionnest, P. Recent Progresses in Mid Infrared Nanocrystal Optoelectronics. IEEE J. Sel. Top. Quantum Electron. 2017, 23, 6000208.

(58) Guyot-Sionnest, P.; Ackerman, M. M.; Tang, X. Colloidal Quantum Dots for Infrared Detection beyond Silicon. J. Chem. Phys. 2019, 151, 060901.

(59) Chen, H.; Li, L.; Cui, S.; Mahounga, D.; Zhang, J.; Gu, Y. Folate Conjugated CdHgTe Quantum Dots with High Targeting Affinity and Sensitivity for In Vivo Early Tumor Diagnosis. J Fluoresc 2011, 21, 793-801.

(60) Schimpf, A. M.; Knowles, K. E.; Carroll, G. M.; Gamelin, D. R. Electronic Doping and Redox-Potential Tuning in Colloidal Semiconductor Nanocrystals. Acc. Chem. Res. 2015, 48, 1929-1937.

(61) Hudson, M. H.; Chen, M.; Kamysbayev, V.; Janke, E. M.; Lan, X.; Allan, G.; Delerue, C.; Lee, B.; Guyot-Sionnest, P.; Talapin, D. V. Conduction Band Fine Structure in Colloidal HgTe Quantum Dots. ACS Nano 2018, 12, 9397-9404.

(62) Moghaddam, N.; Gréboval, C.; Qu, J.; Chu, A.; Rastogi, P.; Livache, C.; Khalili, A.; Xu, X. Z.; Baptiste, B.; Klotz, S.; et al. The Strong Confinement Regime in HgTe Two-Dimentional Nanoplatelets. J. Phys. Chem. C 2020, 124, 23460-23468.

(63) Shen, G.; Chen, M.; Guyot-Sionnest, P. Synthesis of Nonaggregating HgTe Colloidal Quantum Dots and the Emergence of Air-Stable n-Doping. J. Phys. Chem. Lett. 2017, 8, 2224-2228.

(64) Zhang, H.; Guyot-Sionnest, P. Shape-Controlled HgTe Colloidal Quantum Dots and Reduced Spin-Orbit Splitting in the Tetrahedral Shape. J. Phys. Chem. Lett. 2020, 11, 6860-6866.

(65) Kim, J.; Choi, D.; Jeong, K. S. Self-Doped Colloidal Semiconductor Nanocrystals with Intraband Transitions in Steady State. Chem. Commun. 2018, 54, 8435-8445.

(66) Jagtap, A.; Livache, C.; Martinez, B.; Qu, J.; Chu, A.; Gréboval, C.; Goubet, N.; Lhuillier, E. Emergence of Intraband Transitions in Colloidal Nanocrystals. Opt. Mater. Express 2018, 8, 1174-1183.

(67) Nasilowski, M.; Mahler, B.; Lhuillier, E.; Ithurria, S.; Dubertret, B. Two-Dimensional Colloidal Nanocrystals. Chem. Rev. 2016, 116, 10934-10982.

(68) Chen, M.; Guyot-Sionnest, P. Reversible Electrochemistry of Mercury Chalcogenide Colloidal Quantum Dot Films. ACS Nano 2017, 11, 4165-4173.

(69) Rogalski, A. HgCdTe Infrared Detector Material: History, Status and Outlook. Rep. Prog. Phys. 2005, 68, 2267-2336.

(70) Svane, A.; Christensen, N. E.; Cardona, M.; Chantis, A. N.; van Schilfgaarde, M.; Kotani, T. Quasiparticle Band Structures of $\beta$-HgS, HgSe, and HgTe. Phys. Rev. B 2011, 84, 205205.

(71) Delin, A.; Klüner, T. Excitation Spectra and Ground-State Properties from Density-Functional Theory for the Inverted Band-Structure Systems $\beta$-HgS, HgSe, and HgTe. Phys. Rev. B 2002, 66, 035117.

(72) Man, P.; Pan, D. S. Infrared Absorption in HgTe. Phys. Rev. B 1991, 44, 8745-8758.

(73) Tennant, W. E. "Rule 07" Revisited: Still a Good Heuristic Predictor of $p / n$ HgCdTe Photodiode Performance? J. Electron. Mater. 2010, 39, 1030-1035.

(74) Tennant, W. E.; Lee, D.; Zandian, M.; Piquette, E.; Carmody, M. MBE HgCdTe Technology: A Very General Solution to IR Detection, Described by “Rule 07", a Very Convenient Heuristic. J. Electron. Mater. 2008, 37, 1406-1410.

(75) Garcia, G.; Buonsanti, R.; Llordes, A.; Runnerstrom, E. L.; Bergerud, A.; Milliron, D. J. Near-Infrared Spectrally Selective Plasmonic Electrochromic Thin Films. Adv. Opt. Mater. 2013, 1, 215-220.

(76) Qu, J.; Livache, C.; Martinez, B.; Gréboval, C.; Chu, A.; Meriggio, E.; Ramade, J.; Cruguel, H.; Xu, X. Z.; Proust, A.; et al. Transport in ITO Nanocrystals with Short- to Long-Wave Infrared Absorption for Heavy-Metal-Free Infrared Photodetection. ACS Appl. Nano Mater. 2019, 2, 1621-1630.

(77) Hafiz, S. B.; Scimeca, M. R.; Zhao, P.; Paredes, I. J.; Sahu, A.; Ko, D.-K. Silver Selenide Colloidal Quantum Dots for MidWavelength Infrared Photodetection. ACS Appl. Nano Mater. 2019, 2, 1631-1636.

(78) Park, M.; Choi, D.; Choi, Y.; Shin, H.; Jeong, K. S. Mid-Infrared Intraband Transition of Metal Excess Colloidal $\mathrm{Ag}_{2} \mathrm{Se}$ Nanocrystals. ACS Photonics 2018, 5, 1907-1911.

(79) Qu, J.; Goubet, N.; Livache, C.; Martinez, B.; Amelot, D.; Gréboval, C.; Chu, A.; Ramade, J.; Cruguel, H.; Ithurria, S.; et al. Intraband Mid-Infrared Transitions in $\mathrm{Ag}_{2} \mathrm{Se}$ Nanocrystals: Potential and Limitations for Hg-Free Low-Cost Photodetection. J. Phys. Chem. C 2018, 122, 18161-18167.

(80) Liu, W.; Chang, A. Y.; Schaller, R. D.; Talapin, D. V. Colloidal InSb Nanocrystals. J. Am. Chem. Soc. 2012, 134, 20258-20261.

(81) Crisp, R. W.; Grimaldi, G.; Trizio, L. D.; Evers, W. H.; Kirkwood, N.; Kinge, S.; Manna, L.; Siebbeles, L. D. A.; Houtepen, A. J. Selective Antimony Reduction Initiating the Nucleation and Growth of InSb Quantum Dots. Nanoscale 2018, 10, 1111011116.

(82) Yarema, M.; Kovalenko, M. V. Colloidal Synthesis of InSb Nanocrystals with Controlled Polymorphism Using Indium and Antimony Amides. Chem. Mater. 2013, 25, 1788-1792.

(83) Hines, M. A.; Scholes, G. D. Colloidal PbS Nanocrystals with Size-Tunable Near-Infrared Emission: Observation of PostSynthesis Self-Narrowing of the Particle Size Distribution. Adv. Mater. 2003, 15, 1844-1849.

(84) Weidman, M. C.; Beck, M. E.; Hoffman, R. S.; Prins, F.; Tisdale, W. A. Monodisperse, Air-Stable PbS Nanocrystals via Precursor Stoichiometry Control. ACS Nano 2014, 8, 6363-6371.

(85) Shulga, A. G.; Piveteau, L.; Bisri, S. Z.; Kovalenko, M. V.; Loi, M. A. Double Gate PbS Quantum Dot Field-Effect Transistors for Tuneable Electrical Characteristics. Adv. Electron. Mater. 2016, 2, 1500467.

(86) Rauch, T.; Böberl, M.; Tedde, S. F.; Fürst, J.; Kovalenko, M. V.; Hesser, G.; Lemmer, U.; Heiss, W.; Hayden, O. Near-Infrared Imaging with Quantum-Dot-Sensitized Organic Photodiodes. Nat. Photonics 2009, 3, 332-336. 
Konstantatos, G.; Howard, I.; Fischer, A.; Hoogland, S.; Clifford, J.; Klem, E.; Levina, L.; Sargent, E. H. Ultrasensitive Solution-Cast Quantum Dot Photodetectors. Nature 2006, 442, 180-183.

(88) McDonald, S. A.; Konstantatos, G.; Zhang, S.; Cyr, P. W.; Klem, E. J. D.; Levina, L.; Sargent, E. H. Solution-Processed PbS Quantum Dot Infrared Photodetectors and Photovoltaics. Nat. Mater. 2005, 4, 138-142.

(89) Özdemir, O.; Ramiro, I.; Gupta, S.; Konstantatos, G. High Sensitivity Hybrid PbS CQD-TMDC Photodetectors up to $2 \mu \mathrm{m}$. ACS Photonics 2019, 6, 2381-2386.

(90) Killilea, N.; Wu, M.; Sytnyk, M.; Amin, A. A. Y.; Mashkov, O.; Spiecker, E.; Heiss, W. Pushing PbS/Metal-Halide-Perovskite Core/Epitaxial-Ligand-Shell Nanocrystal Photodetectors beyond $3 \mu \mathrm{m}$ Wavelength. Adv. Funct. Mater. 2019, $29,1807964$.

(91) Günes, S.; Neugebauer, H.; Sariciftci, N. S.; Roither, J.; Kovalenko, M.; Pillwein, G.; Heiss, W. Hybrid Solar Cells Using HgTe Nanocrystals and Nanoporous $\mathrm{TiO}_{2}$ Electrodes. Adv. Funct. Mater. 2006, 16, 1095-1099.

(92) Kershaw, S. V.; Yiu, W. K.; Sergeev, A.; Rogach, A. L. Development of Synthetic Methods to Grow Long-Wavelength Infrared-Emitting HgTe Quantum Dots in Dimethylformamide. Chem. Mater. 2020, 32, 3930-3943.

(93) Green, M.; Wakefield, G.; Dobson, P. J. A Simple Metalorganic Route to Organically Passivated Mercury Telluride Nanocrystals. J. Mater. Chem. 2003, 13, 1076-1078.

(94) Piepenbrock, M.-O. M.; Stirner, T.; Kelly, S. M.; O'Neill, M. A Low-Temperature Synthesis for Organically Soluble HgTe Nanocrystals Exhibiting Near-Infrared Photoluminescence and Quantum Confinement. J. Am. Chem. Soc. 2006, 128, 7087-7090.

(95) Keuleyan, S.; Lhuillier, E.; Guyot-Sionnest, P. Synthesis of Colloidal HgTe Quantum Dots for Narrow Mid-IR Emission and Detection. J. Am. Chem. Soc. 2011, 133, 16422-16424.

(96) Goubet, N.; Thomas, M.; Gréboval, C.; Chu, A.; Qu, J.; Rastogi, P.; Chee, S.-S.; Goyal, M.; Zhang, Y.; Xu, X. Z.; et al. Nearto Long-Wave-Infrared Mercury Chalcogenide Nanocrystals from Liquid Mercury. J. Phys. Chem. C 2020, 124, 8423-8430.

(97) Müllenborn, M.; Jarvis, R. F.; Yacobi, B. G.; Kaner, R. B.; Coleman, C. C.; Haegel, N. M. Characterization of SolutionSynthesized CdTe and HgTe. Appl. Phys. A 1993, 56, 317-321.

(98) Rogach, A. L.; Katsikas, L.; Kornowski, A.; Su, D.; Eychmüller, A.; Weller, H. Synthesis and Characterization of ThiolStabilized CdTe Nanocrystals. Ber. Bunsenges. Phys. Chem. 1996, 100, 1772-1778.

(99) Gao, M.; Kirstein, S.; Möhwald, H.; Rogach, A. L.; Kornowski, A.; Eychmüller, A.; Weller, H. Strongly Photoluminescent CdTe Nanocrystals by Proper Surface Modification. J. Phys. Chem. B 1998, 102, 8360-8363.

(100) Harrison, M. T.; Kershaw, S. V.; Burt, M. G.; Rogach, A.; Eychmüller, A.; Weller, H. Investigation of Factors Affecting the Photoluminescence of Colloidally-Prepared HgTe Nanocrystals. J. Mater. Chem. 1999, 9, 2721-2722.

(101) Abdelazim, N. M.; Zhu, Q.; Xiong, Y.; Zhu, Y.; Chen, M.; Zhao, N.; Kershaw, S. V.; Rogach, A. L. Room Temperature Synthesis of HgTe Quantum Dots in an Aprotic Solvent Realizing High Photoluminescence Quantum Yields in the Infrared. Chem. Mater. 2017, 29, 7859-7867.

(102) Wang, Y.; Liu, Z.; Huo, N.; Li, F.; Gu, M.; Ling, X.; Zhang, Y.; Lu, K.; Han, L.; Fang, H.; et al. Room-Temperature Direct Synthesis of Semi-Conductive PbS Nanocrystal Inks for Optoelectronic Applications. Nat. Commun. 2019, 10, 5136.

(103) Kim, H.; Park, B.; Cho, K.; Kim, J.-H.; Lee, J. W.; Kim, D.-W.; Kim, S. Transport of Charge Carriers in HgTe/CdTe Core-Shell Nanoparticle Film. Jpn. J. Appl. Phys. 2005, 44, 5703.

(104) Endicott, K. The Trembling Edge of Science. Dartmouth Alumni Magazine 1998, 22-31.

(105) Green, M. Production of Metal Chalcogenide Nanoparticles. US20040086444A1, May 6, 2004.

(106) Kim, S.; Kim, T.; Im, S. H.; Seok, S. I.; Kim, K. W.; Kim, S.; Kim, S.-W. Bandgap Engineered Monodisperse and Stable Mercury Telluride Quantum Dots and Their Application for Near-Infrared Photodetection. J. Mater. Chem. 2011, 21, 15232-15236.

(107) Li, L. S.; Wang, H.; Liu, Y.; Lou, S.; Wang, Y.; Du, Z. Room Temperature Synthesis of HgTe Nanocrystals. J. Colloid Interface Sci. 2007, 308, 254-257.

(108) Noumbé, U. N.; Gréboval, C.; Livache, C.; Chu, A.; Majjad, H.; Parra López, L. E.; Mouafo, L. D. N.; Doudin, B.; Berciaud, S.; Chaste, J.; et al. Reconfigurable 2D/OD p-n Graphene/HgTe Nanocrystal Heterostructure for Infrared Detection. ACS Nano 2020, 14, 4567-4576.

(109) Grigel, V.; Sagar, L. K.; De Nolf, K.; Zhao, Q.; Vantomme, A.; De Roo, J.; Infante, I.; Hens, Z. The Surface Chemistry of Colloidal HgSe Nanocrystals, toward Stoichiometric Quantum Dots by Design. Chem. Mater. 2018, 30, 7637-7647.

(110) Pan, Y.; Bai, H.; Pan, L.; Li, Y.; Tamargo, M. C.; Sohel, M.; Lombardi, J. R. Size Controlled Synthesis of Monodisperse PbTe Quantum Dots: Using Oleylamine as the Capping Ligand. J. Mater. Chem. 2012, 22, 23593-23601.

(111) Wehrenberg, B. L.; Wang, C.; Guyot-Sionnest, P. Interband and Intraband Optical Studies of PbSe Colloidal Quantum Dots. J. Phys. Chem. B 2002, 106, 10634-10640.

(112) Rinnerbauer, V.; Hingerl, K.; Kovalenko, M.; Heiss, W. Effect of Quantum Confinement on Higher Transitions in HgTe Nanocrystals. Appl. Phys. Lett. 2006, 89, 193114.

(113) Jean, J.; Xiao, J.; Nick, R.; Moody, N.; Nasilowski, M.; Bawendi, M.; Bulović, V. Synthesis Cost Dictates the Commercial Viability of Lead Sulfide and Perovskite Quantum Dot Photovoltaics. Energy Environ. Sci. 2018, 11, 2295-2305.

(114) Lhuillier, E.; Keuleyan, S.; Guyot-Sionnest, P. Optical Properties of HgTe Colloidal Quantum Dots. Nanotechnology 2012, 23, 175705.

(115) Harrison, M. T.; Kershaw, S. V.; Burt, M. G.; Rogach, A. L.; Kornowski, A.; Eychmüller, A.; Weller, H. Colloidal Nanocrystals for Telecommunications. Complete Coverage of the Low-Loss Fiber Windows by Mercury Telluride Quantum Dot. Pure Appl. Chem. 2000, 72, 295-307. 
(116) Kuno, M.; Higginson, K. A.; Qadri, S. B.; Yousuf, M.; Lee, S. H.; Davis, B. L.; Mattoussi, H. Molecular Clusters of Binary and Ternary Mercury Chalcogenides: Colloidal Synthesis, Characterization, and Optical Spectra. J. Phys. Chem. B 2003, 107, 5758-5767.

(117) Howes, P.; Green, M.; Johnston, C.; Crossley, A. Synthesis and Shape Control of Mercury Selenide (HgSe) Quantum Dots. J. Mater. Chem. 2008, 18, 3474-3480.

(118) Jeong, J.; Yoon, B.; Kwon, Y.-W.; Choi, D.; Jeong, K. S. Singly and Doubly Occupied Higher Quantum States in Nanocrystals. Nano Lett. 2017, 17, 1187-1193.

(119) Choi, D.; Park, M.; Jeong, J.; Shin, H.-B.; Choi, Y. C.; Jeong, K. S. Multifunctional Self-Doped Nanocrystal Thin-Film Transistor Sensors. ACS Appl. Mater. Interfaces 2019, 11, 7242-7249.

(120) Goubet, N.; Livache, C.; Martinez, B.; Xu, X. Z.; Ithurria, S.; Royer, S.; Cruguel, H.; Patriarche, G.; Ouerghi, A.; Silly, M.; et al. Wave-Function Engineering in $\mathrm{HgSe} / \mathrm{HgTe}$ Colloidal Heterostructures To Enhance Mid-Infrared Photoconductive Properties. Nano Lett. 2018, 18, 4590-4597.

(121) Chen, M.; Shen, G.; Guyot-Sionnest, P. Size Distribution Effects on Mobility and Intraband Gap of HgSe Quantum Dots. J. Phys. Chem. C 2020, 124, 16216-16221.

(122) Martinez, B.; Livache, C.; Notemgnou Mouafo, L. D.; Goubet, N.; Keuleyan, S.; Cruguel, H.; Ithurria, S.; Aubin, H.; Ouerghi, A.; Doudin, B.; et al. HgSe Self-Doped Nanocrystals as a Platform to Investigate the Effects of Vanishing Confinement. ACS Appl. Mater. Interfaces 2017, 9, 36173-36180.

(123) A. Gonzales, M.; Han, H.; Moyes, A.; Radinos, A.; J. Hobbs, A.; Coombs, N.; J. Oliver, S. R.; K. Mascharak, P. Light-Triggered Carbon Monoxide Delivery with Al-MCM-41-Based Nanoparticles Bearing a Designed Manganese Carbonyl Complex. J. Mater. Chem. B 2014, 2, 2107-2113.

(124) Choi, D.; Yoon, B.; Kim, D.-K.; Baik, H.; Choi, J.-H.; Jeong, K. S. Major Electronic Transition Shift from Bandgap to Localized Surface Plasmon Resonance in $\mathrm{Cd}_{\mathrm{x}} \mathrm{Hg}_{1-\mathrm{x}} \mathrm{Se}$ Alloy Nanocrystals. Chem. Mater. 2017, 29, 8548-8554.

(125) Schooss, D.; Mews, A.; Eychmüller, A.; Weller, H. Quantum-Dot Quantum Well CdS/HgS/CdS: Theory and Experiment. Phys. Rev. B 1994, 49, 17072-17078.

(126) Kamalov, V. F.; Little, R.; Logunov, S. L.; El-Sayed, M. A. Picosecond Electronic Relaxation in CdS/HgS/CdS Quantum Dot Quantum Well Semiconductor Nanoparticles. J. Phys. Chem. 1996, 100, 6381-6384.

(127) Mews, A.; Kadavanich, A. V.; Banin, U.; Alivisatos, A. P. Structural and Spectroscopic Investigations of CdS/HgS/CdS Quantum-Dot Quantum Wells. Phys. Rev. B 1996, 53, R13242-R13245.

(128) Dorfs, D.; Henschel, H.; Kolny, J.; Eychmüller, A. Multilayered Nanoheterostructures: Theory and Experiment. J. Phys. Chem. B 2004, 108, 1578-1583.

(129) Higginson, K. A.; Kuno, M.; Bonevich, J.; Qadri, S. B.; Yousuf, M.; Mattoussi, H. Synthesis and Characterization of Colloidal B-HgS Quantum Dots. J. Phys. Chem. B 2002, 106, 9982-9985.

(130) Yang, Y.-G.; Liu, H.-G.; Chen, L.-J.; Chen, K.-C.; Ding, H.-P.; Hao, J. One-Step Synthesis and Assembly of Two-Dimensional Arrays of Mercury Sulfide Nanocrystal Aggregates at the Air/Water Interface. Langmuir 2010, 26, 14879-14884.

(131) Wichiansee, W.; N. Nordin, M.; Green, M.; J. Curry, R. Synthesis and Optical Characterization of Infra-Red Emitting Mercury Sulfide (HgS) Quantum Dots. J. Mater. Chem. 2011, 21, 7331-7336.

(132) Yang, J.; Zhang, W.-H.; Hu, Y.-P.; Yu, J.-S. Aqueous Synthesis and Characterization of Glutathione-Stabilized $\beta$-HgS Nanocrystals with near-Infrared Photoluminescence. J. Colloid Interface Sci. 2012, 379, 8-13.

(133) Zhang, L.; Yang, G.; He, G.; Wang, L.; Liu, Q.; Zhang, Q.; Qin, D. Synthesis of HgS Nanocrystals in the Lysozyme Aqueous Solution through Biomimetic Method. Appl. Surf. Sci. 2012, 258, 8185-8191.

(134) Goswami, N.; Giri, A.; Kar, S.; Bootharaju, M. S.; John, R.; Xavier, P. L.; Pradeep, T.; Pal, S. K. Protein-Directed Synthesis of NIR-Emitting, Tunable HgS Quantum Dots and Their Applications in Metal-lon Sensing. Small 2012, 8, 3175-3184.

(135) Han, L.; Hou, P.; Feng, Y.; Liu, H.; Li, J.; Peng, Z.; Yang, J. Phase Transfer-Based Synthesis of HgS Nanocrystals. Dalton Trans. 2014, 43, 11981-11987.

(136) Shen, G.; Guyot-Sionnest, P. HgS and HgS/CdS Colloidal Quantum Dots with Infrared Intraband Transitions and Emergence of a Surface Plasmon. J. Phys. Chem. C 2016, 120, 11744-11753.

(137) Mews, A.; Eychmueller, A.; Giersig, M.; Schooss, D.; Weller, H. Preparation, characterization, and photophysics of the quantum dot quantum well system cadmium sulfide/mercury sulfide/cadmium sulfide. J. Phys. Chem. 1994, 98, 934-941.

(138) Ben-Moshe, A.; Govorov, A. O.; Markovich, G. Enantioselective Synthesis of Intrinsically Chiral Mercury Sulfide Nanocrystals. Angew. Chem. 2013, 125, 1313-1317.

(139) Wang, P.; Yu, S.-J.; Govorov, A. O.; Ouyang, M. Cooperative Expression of Atomic Chirality in Inorganic Nanostructures. Nat. Commun. 2017, 8, 14312.

(140) Kuno, J.; Imamura, Y.; Katouda, M.; Tashiro, M.; Kawai, T.; Nakashima, T. Inversion of Optical Activity in the Synthesis of Mercury Sulfide Nanoparticles: Role of Ligand Coordination. Angew. Chem. Int. Ed. 2018, 57, 12022-12026.

(141) Kuno, J.; Kawai, T.; Nakashima, T. The Effect of Surface Ligands on the Optical Activity of Mercury Sulfide Nanoparticles. Nanoscale 2017, 9, 11590-11595.

(142) Wang, P.; Yu, S.-J.; Ouyang, M. Assembled Suprastructures of Inorganic Chiral Nanocrystals and Hierarchical Chirality. J. Am. Chem. Soc. 2017, 139, 6070-6073.

(143) Manna, L.; Milliron, D. J.; Meisel, A.; Scher, E. C.; Alivisatos, A. P. Controlled Growth of Tetrapod-Branched Inorganic Nanocrystals. Nat. Mater. 2003, 2, 382-385. 
(144) Ithurria, S.; Dubertret, B. Quasi 2D Colloidal CdSe Platelets with Thicknesses Controlled at the Atomic Level. J. Am. Chem. Soc. 2008, 130, 16504-16505.

(145) Zhou, G.; Lü, M.; Xiu, Z.; Wang, S.; Zhang, H.; Zhou, Y.; Wang, S. Controlled Synthesis of High-Quality PbS Star-Shaped Dendrites, Multipods, Truncated Nanocubes, and Nanocubes and Their Shape Evolution Process. J. Phys. Chem. B 2006, 110, 6543-6548.

(146) Martinez, B.; Ramade, J.; Livache, C.; Goubet, N.; Chu, A.; Gréboval, C.; Qu, J.; Watkins, W. L.; Becerra, L.; Dandeu, E.; et al. HgTe Nanocrystal Inks for Extended Short-Wave Infrared Detection. Adv. Opt. Mater. 2019, 7, 1900348.

(147) Riedinger, A.; Ott, F. D.; Mule, A.; Mazzotti, S.; Knüsel, P. N.; Kress, S. J. P.; Prins, F.; Erwin, S. C.; Norris, D. J. An Intrinsic Growth Instability in Isotropic Materials Leads to Quasi-Two-Dimensional Nanoplatelets. Nat. Mater. 2017, 16, 743-748.

(148) Ithurria, S.; Bousquet, G.; Dubertret, B. Continuous Transition from 3D to 1D Confinement Observed during the Formation of CdSe Nanoplatelets. J. Am. Chem. Soc. 2011, 133, 3070-3077.

(149) Tessier, M. D.; Javaux, C.; Maksimovic, I.; Loriette, V.; Dubertret, B. Spectroscopy of Single CdSe Nanoplatelets. ACS Nano 2012, 6, 6751-6758.

(150) Grim, J. Q.; Christodoulou, S.; Di Stasio, F.; Krahne, R.; Cingolani, R.; Manna, L.; Moreels, I. Continuous-Wave Biexciton Lasing at Room Temperature Using Solution-Processed Quantum Wells. Nat. Nanotechnol. 2014, 9, 891-895.

(151) She, C.; Fedin, I.; Dolzhnikov, D. S.; Demortière, A.; Schaller, R. D.; Pelton, M.; Talapin, D. V. Low-Threshold Stimulated Emission Using Colloidal Quantum Wells. Nano Lett. 2014, 14, 2772-2777.

(152) Guzelturk, B.; Kelestemur, Y.; Olutas, M.; Delikanli, S.; Demir, H. V. Amplified Spontaneous Emission and Lasing in Colloidal Nanoplatelets. ACS Nano 2014, 8, 6599-6605.

(153) Liu, B.; Altintas, Y.; Wang, L.; Shendre, S.; Sharma, M.; Sun, H.; Mutlugun, E.; Demir, H. V. Record High External Quantum Efficiency of 19.2\% Achieved in Light-Emitting Diodes of Colloidal Quantum Wells Enabled by Hot-Injection Shell Growth. Adv. Mater. 2020, 32, 1905824.

(154) Chen, Z.; Nadal, B.; Mahler, B.; Aubin, H.; Dubertret, B. Quasi-2D Colloidal Semiconductor Nanoplatelets for Narrow Electroluminescence. Adv. Funct. Mater. 2014, 24, 295-302.

(155) Kelestemur, Y.; Shynkarenko, Y.; Anni, M.; Yakunin, S.; De Giorgi, M. L.; Kovalenko, M. V. Colloidal CdSe Quantum Wells with Graded Shell Composition for Low-Threshold Amplified Spontaneous Emission and Highly Efficient Electroluminescence. ACS Nano 2019, 13, 13899-13909.

(156) Qu, J.; Rastogi, P.; Greboval, C.; Livache, C.; Dufour, M.; Chu, A.; Chee, S.-S.; Ramade, J.; Xu, X. Z.; Ithurria, S.; et al. Nanoplatelet-Based Light Emitting Diode and Its Use for All-Nanocrystal LiFi-like Communication. ACS Appl. Mater. Interfaces 2020,12, 22058-22065.

(157) Diroll, B. T. Colloidal Quantum Wells for Optoelectronic Devices. J. Mater. Chem. C 2020, 8, $10628-10640$.

(158) Lhuillier, E.; Ithurria, S.; Descamps-Mandine, A.; Douillard, T.; Castaing, R.; Xu, X. Z.; Taberna, P.-L.; Simon, P.; Aubin, H.; Dubertret, B. Investigating the $\mathrm{n}$ - and p-Type Electrolytic Charging of Colloidal Nanoplatelets. J. Phys. Chem. C 2015, 119, 21795-21799.

(159) Lhuillier, E.; Dayen, J.-F.; Thomas, D. O.; Robin, A.; Doudin, B.; Dubertret, B. Nanoplatelets Bridging a Nanotrench: A New Architecture for Photodetectors with Increased Sensitivity. Nano Lett. 2015, 15, 1736-1742.

(160) Schliehe, C.; Juarez, B. H.; Pelletier, M.; Jander, S.; Greshnykh, D.; Nagel, M.; Meyer, A.; Foerster, S.; Kornowski, A.; Klinke, C.; et al. Ultrathin PbS Sheets by Two-Dimensional Oriented Attachment. Science 2010, 329, 550-553.

(161) Pedetti, S.; Nadal, B.; Lhuillier, E.; Mahler, B.; Bouet, C.; Abécassis, B.; Xu, X.; Dubertret, B. Optimized Synthesis of CdTe Nanoplatelets and Photoresponse of CdTe Nanoplatelets Films. Chem. Mater. 2013, 25, 2455-2462.

(162) Galle, T.; Samadi Khoshkhoo, M.; Martin-Garcia, B.; Meerbach, C.; Sayevich, V.; Koitzsch, A.; Lesnyak, V.; Eychmüller, A. Colloidal PbSe Nanoplatelets of Varied Thickness with Tunable Optical Properties. Chem. Mater. 2019, 31, 3803-3811.

(163) Galle, T.; Kazes, M.; Hübner, R.; Lox, J.; Samadi Khoshkhoo, M.; Sonntag, L.; Tietze, R.; Sayevich, V.; Oron, D.; Koitzsch, A.; et al. Colloidal Mercury-Doped CdSe Nanoplatelets with Dual Fluorescence. Chem. Mater. 2019, 31, 5065-5074.

(164) Peterson, J. J.; Krauss, T. D. Fluorescence Spectroscopy of Single Lead Sulfide Quantum Dots. Nano Lett. 2006, 6, 510514.

(165) Gréboval, C.; Izquierdo, E.; Livache, C.; Martinez, B.; Dufour, M.; Goubet, N.; Moghaddam, N.; Qu, J.; Chu, A.; Ramade, J.; et al. Impact of Dimensionality and Confinement on the Electronic Properties of Mercury Chalcogenide Nanocrystals. Nanoscale 2019, 11, 3905-3915.

(166) Izquierdo, E.; Dufour, M.; Chu, A.; Livache, C.; Martinez, B.; Amelot, D.; Patriarche, G.; Lequeux, N.; Lhuillier, E.; Ithurria, S. Coupled HgSe Colloidal Quantum Wells through a Tunable Barrier: A Strategy To Uncouple Optical and Transport Band Gap. Chem. Mater. 2018, 30, 4065-4072.

(167) Smith, A. M.; Lane, L. A.; Nie, S. Mapping the Spatial Distribution of Charge Carriers in Quantum-Confined Heterostructures. Nat. Commun. 2014, 5, 4506.

(168) Keuleyan, S.; Kohler, J.; Guyot-Sionnest, P. Photoluminescence of Mid-Infrared HgTe Colloidal Quantum Dots. J. Phys. Chem. C 2014, 118, 2749-2753.

(169) Deng, Z.; Guyot-Sionnest, P. Intraband Luminescence from HgSe/CdS Core/Shell Quantum Dots. ACS Nano 2016, 10, 2121-2127.

(170) Kershaw, S. V.; Harrison, M.; Rogach, A. L.; Kornowski, A. Development of IR-Emitting Colloidal II-VI Quantum-Dot Materials. IEEE J. Sel. Top. Quantum Electron. 2000, 6, 534-543. 
(171) Lifshitz, E.; Porteanu, H.; Glozman, A.; Weller, H.; Pflughoefft, M.; Echymüller, A. Optically Detected Magnetic Resonance Study of CdS/HgS/CdS Quantum Dot Quantum Wells. J. Phys. Chem. B 1999, 103, 6870-6875.

(172) Ecyhmüller, A.; Voßmeyer, T.; Mews, A.; Weller, H. Transient Photobleaching in the Quantum Dot Quantum Well $\mathrm{CdS} / \mathrm{HgS} / \mathrm{CdS}$. J. Lumin. 1994, 58, 223-226.

(173) Mews, A.; Eychmüller, A. Quantum Wells within Quantum Dots, a CdS/HgS Nanoheterostructure with Global and Local Confinement. Ber. Bunsenges. Physik. Chem. 1998, 102, 1343-1357.

(174) Porţeanu, H. E.; Lifshitz, E.; Pflughoefft, M.; Eychmüller, A.; Weller, H. Optical Properties of CdS/HgS/CdS Quantum DotQuantum Well Structures. Phys. Stat. Solidi. B 2001, 226, 219-232.

(175) Smith, A. M.; Nie, S. Bright and Compact Alloyed Quantum Dots with Broadly Tunable Near-Infrared Absorption and Fluorescence Spectra through Mercury Cation Exchange. J. Am. Chem. Soc. 2011, 133, 24-26.

(176) Kershaw, S. V.; Burt, M.; Harrison, M.; Rogach, A.; Weller, H.; Eychmüller, A. Colloidal CdTe/HgTe Quantum Dots with High Photoluminescence Quantum Efficiency at Room Temperature. Appl. Phys. Lett. 1999, 75, 1694-1696.

(177) Pietryga, J. M.; Werder, D. J.; Williams, D. J.; Casson, J. L.; Schaller, R. D.; Klimov, V. I.; Hollingsworth, J. A. Utilizing the Lability of Lead Selenide to Produce Heterostructured Nanocrystals with Bright, Stable Infrared Emission. J. Am. Chem. Soc. 2008, 130, 4879-4885.

(178) Lambert, K.; Geyter, B. D.; Moreels, I.; Hens, Z. PbTe|CdTe Core|Shell Particles by Cation Exchange, a HR-TEM Study. Chem. Mater. 2009, 21, 778-780.

(179) Zhou, H. S.; Honma, I.; Komiyama, H.; Haus, J. W. Coated Semiconductor Nanoparticles; the Cadmium Sulfide/Lead Sulfide System's Synthesis and Properties. J. Phys. Chem. 1993, 97, 895-901.

(180) Justo, Y.; Geiregat, P.; van Hoecke, K.; Vanhaecke, F.; De Mello Donega, C.; Hens, Z. Optical Properties of PbS/CdS Core/Shell Quantum Dots. J. Phys. Chem. C 2013, 117, 20171-20177.

(181) Ithurria, S.; Talapin, D. V. Colloidal Atomic Layer Deposition (c-ALD) Using Self-Limiting Reactions at Nanocrystal Surface Coupled to Phase Transfer between Polar and Nonpolar Media. J. Am. Chem. Soc. 2012, 134, 18585-18590.

(182) Hazarika, A.; Fedin, I.; Hong, L.; Guo, J.; Srivastava, V.; Cho, W.; Coropceanu, I.; Portner, J.; Diroll, B. T.; Philbin, J. P.; et al. Colloidal Atomic Layer Deposition with Stationary Reactant Phases Enables Precise Synthesis of "Digital" II-VI NanoHeterostructures with Exquisite Control of Confinement and Strain. J. Am. Chem. Soc. 2019, 141, 13487-13496.

(183) Lhuillier, E.; Pedetti, S.; Ithurria, S.; Nadal, B.; Heuclin, H.; Dubertret, B. Two-Dimensional Colloidal Metal Chalcogenides Semiconductors: Synthesis, Spectroscopy, and Applications. Acc. Chem. Res. 2015, 48, 22-30.

(184) Li, J. J.; Wang, Y. A.; Guo, W.; Keay, J. C.; Mishima, T. D.; Johnson, M. B.; Peng, X. Large-Scale Synthesis of Nearly Monodisperse CdSe/CdS Core/Shell Nanocrystals Using Air-Stable Reagents via Successive Ion Layer Adsorption and Reaction. J. Am. Chem. Soc. 2003, 125, 12567-12575.

(185) Sagar, L. K.; Walravens, W.; Maes, J.; Geiregat, P.; Hens, Z. HgSe/CdE (E = S, Se) Core/Shell Nanocrystals by Colloidal Atomic Layer Deposition. J. Phys. Chem. C 2017, 121, 13816-13822.

(186) Robin, A.; Livache, C.; Ithurria, S.; Lacaze, E.; Dubertret, B.; Lhuillier, E. Surface Control of Doping in Self-Doped Nanocrystals. ACS Appl. Mater. Interfaces 2016, 8, 27122-27128.

(187) Shen, G.; Guyot-Sionnest, P. HgTe/CdTe and HgSe/CdX (X = S, Se, and Te) Core/Shell Mid-Infrared Quantum Dots. Chem. Mater. 2019, 31, 286-293.

(188) Lhuillier, E.; Pedetti, S.; Ithurria, S.; Heuclin, H.; Nadal, B.; Robin, A.; Patriarche, G.; Lequeux, N.; Dubertret, B. ElectrolyteGated Field Effect Transistor to Probe the Surface Defects and Morphology in Films of Thick CdSe Colloidal Nanoplatelets. ACS Nano 2014, 8, 3813-3820.

(189) Chu, A.; Livache, C.; Ithurria, S.; Lhuillier, E. Electronic Structure Robustness and Design Rules for 2D Colloidal Heterostructures. J. Appl. Phys. 2018, 123, 035701.

(190) Rossinelli, A. A.; Riedinger, A.; Marqués-Gallego, P.; Knüsel, P. N.; Antolinez, F. V.; Norris, D. J. High-Temperature Growth of Thick-Shell CdSe/CdS Core/Shell Nanoplatelets. Chem. Commun. 2017, 53, 9938-9941.

(191) Piveteau, L.; Dirin, D.; Gordon, C. P.; Walder, B. J.; Ong, T.-C.; Emsley, L.; Copéret, C.; Kovalenko, M. V. Colloidal-ALDGrown Core/Shell CdSe/CdS Nanoplatelets as Seen by DNP Enhanced PASS-PIETA NMR Spectroscopy. Nano Lett. 2020, 20, 3003-3018.

(192) Jagtap, A.; Martinez, B.; Goubet, N.; Chu, A.; Livache, C.; Gréboval, C.; Ramade, J.; Amelot, D.; Trousset, P.; Triboulin, A.; et al. Design of a Unipolar Barrier for a Nanocrystal-Based Short-Wave Infrared Photodiode. ACS Photonics 2018, 5, 45694576.

(193) Yoon, B.; Jeong, J.; Jeong, K. S. Higher Quantum State Transitions in Colloidal Quantum Dot with Heavy Electron Doping. J. Phys. Chem. C 2016, 120, 22062-22068.

(194) Kim, J.; Yoon, B.; Kim, J.; Choi, Y.; Kwon, Y.-W.; Park, S. K.; Jeong, K. S. High Electron Mobility of $\beta$-HgS Colloidal Quantum Dots with Doubly Occupied Quantum States. RSC Adv. 2017, 7, 38166-38170.

(195) Brown, P. R.; Kim, D.; Lunt, R. R.; Zhao, N.; Bawendi, M. G.; Grossman, J. C.; Bulović, V. Energy Level Modification in Lead Sulfide Quantum Dot Thin Films through Ligand Exchange. ACS Nano 2014, 8, 5863-5872.

(196) Ganesan, A. A.; Houtepen, A. J.; Crisp, R. W. Quantum Dot Solar Cells: Small Beginnings Have Large Impacts. Appl. Sci. 2018, 8, 1867.

(197) Martinez, B.; Plamont, R.; Gréboval, C.; Rastogi, P.; Prado, Y.; Qu, J.; Chu, A.; Livache, C.; Xu, X. Z.; Cruguel, H.; et al. Azobenzenes as Light-Activable Carrier Density Switches in Nanocrystals. J. Phys. Chem. C 2019, 123, $27257-27263$.

(198) Hartley, G. S. The Cis -Form of Azobenzene. Nature 1937, 140, 281-281. 
Beharry, A. A.; Woolley, G. A. Azobenzene Photoswitches for Biomolecules. Chem. Soc. Rev. 2011, 40, $4422-4437$. Kumar, G. S.; Neckers, D. C. Photochemistry of Azobenzene-Containing Polymers. Chem. Rev. 1989, 89, $1915-1925$. Samanta, D.; Gemen, J.; Chu, Z.; Diskin-Posner, Y.; Shimon, L. J. W.; Klajn, R. Reversible Photoswitching of Encapsulated Azobenzenes in Water. Proc. Natl. Acad. Sci. 2018, 115, 9379-9384.

Credi, A. Artificial Molecular Motors Powered by Light. Aust. J. Chem. 2006, 59, 157-169.

Hartley, G. S.; Fèvre, R. J. W. L. 119. The Dipole Moments of Cis- and Trans-Azobenzenes and of Some Related Compounds. J. Chem. Soc. 1939, 1939, 531-535.

Martinez, B.; Livache, C.; Meriggio, E.; Xu, X. Z.; Cruguel, H.; Lacaze, E.; Proust, A.; Ithurria, S.; Silly, M. G.; Cabailh, G.; et al. Polyoxometalate as Control Agent for the Doping in HgSe Self-Doped Nanocrystals. J. Phys. Chem. C 2018, 122, 2668026685.

Huang, J.; Liu, W.; Dolzhnikov, D. S.; Protesescu, L.; Kovalenko, M. V.; Koo, B.; Chattopadhyay, S.; Shenchenko, E. V.; Talapin, D. V. Surface Functionalization of Semiconductor and Oxide Nanocrystals with Small Inorganic Oxoanions (PO ${ }^{3-}$ , $\left.\mathrm{MoO}_{4}{ }^{2-}\right)$ and Polyoxometalate Ligands. ACS Nano 2014, 8, 9388-9402.

Liu, H.; Guyot-Sionnest, P. Magnetoresistance of Manganese-Doped Colloidal Quantum Dot Films. J. Phys. Chem. C 2015, 119, 14797-14804.

Yang, H.; Wong, E.; Zhao, T.; Lee, J. D.; Xin, H. L.; Chi, M.; Fleury, B.; Tang, H.-Y.; Gaulding, E. A.; Kagan, C. R.; et al. Charge Transport Modulation in PbSe Nanocrystal Solids by AuxAg1-x Nanoparticle Doping. ACS Nano 2018, 12, 9091-9100.

Cargnello, M.; Johnston-Peck, A. C.; Diroll, B. T.; Wong, E.; Datta, B.; Damodhar, D.; Doan-Nguyen, V. V. T.; Herzing, A. A.; Kagan, C. R.; Murray, C. B. Substitutional Doping in Nanocrystal Superlattices. Nature 2015, 524, 450-453.

Groves, S. H.; Brown, R. N.; Pidgeon, C. R. Interband Magnetoreflection and Band Structure of HgTe. Phys. Rev. 1967, 161, 779-793.

Groves, S.; Paul, W. Band Structure of Gray Tin. Phys. Rev. Lett. 1963, 11, 194-196.

Bendias, K.; Shamim, S.; Herrmann, O.; Budewitz, A.; Shekhar, P.; Leubner, P.; Kleinlein, J.; Bocquillon, E.; Buhmann, H.; Molenkamp, L. W. High Mobility HgTe Microstructures for Quantum Spin Hall Studies. Nano Lett. 2018, 18, 4831-4836.

Moon, C.-Y.; Wei, S.-H. Band Gap of Hg Chalcogenides: Symmetry-Reduction-Induced Band-Gap Opening of Materials with Inverted Band Structures. Phys. Rev. B 2006, 74, 045205.

Madelung, O. Semiconductors: Data Handbook; Springer Science \& Business Media, 2012.

Pokatilov, E. P.; Fonoberov, V. A.; Fomin, V. M.; Devreese, J. T. Electron and Hole States in Quantum Dot Quantum Wells within a Spherical Eight-Band Model. Phys. Rev. B 2001, 64, 245329.

Beugeling, W.; Kalesaki, E.; Delerue, C.; Niquet, Y.-M.; Vanmaekelbergh, D.; Smith, C. M. Topological States in MultiOrbital HgTe Honeycomb Lattices. Nat. Commun. 2015, 6, 6316.

Allan, G.; Delerue, C. Tight-Binding Calculations of the Optical Properties of HgTe Nanocrystals. Phys. Rev. B 2012, 86, 165437.

Willardson, R. K.; Beer, A. C. Semiconductors and Semimetals; Academic Press, 1977, 18.

Al-Otaify, A.; Kershaw, S. V.; Gupta, S.; Rogach, A. L.; Allan, G.; Delerue, C.; Binks, D. J. Multiple Exciton Generation and Ultrafast Exciton Dynamics in HgTe Colloidal Quantum Dots. Phys. Chem. Chem. Phys. 2013, 15, 16864-16873.

Livache, C.; Goubet, N.; Martinez, B.; Jagtap, A.; Qu, J.; Ithurria, S.; Silly, M. G.; Dubertret, B.; Lhuillier, E. Band Edge Dynamics and Multiexciton Generation in Narrow Band Gap HgTe Nanocrystals. ACS Appl. Mater. Interfaces 2018, 10, 11880-11887.

Evers, W. H.; Goris, B.; Bals, S.; Casavola, M.; de Graaf, J.; van Roij, R.; Dijkstra, M.; Vanmaekelbergh, D. Low-Dimensional Semiconductor Superlattices Formed by Geometric Control over Nanocrystal Attachment. Nano Lett. 2013, 13, 23172323.

Geuchies, J. J.; van Overbeek, C.; Evers, W. H.; Goris, B.; de Backer, A.; Gantapara, A. P.; Rabouw, F. T.; Hilhorst, J.; Peters, J. L.; Konovalov, O.; et al. In Situ Study of the Formation Mechanism of Two-Dimensional Superlattices from PbSe Nanocrystals. Nat. Mater. 2016, 15, 1248-1254.

Boneschanscher, M. P.; Evers, W. H.; Geuchies, J. J.; Altantzis, T.; Goris, B.; Rabouw, F. T.; Rossum, S. A. P. van; Zant, H. S. J. van der; Siebbeles, L. D. A.; Tendeloo, G. V.; et al. Long-Range Orientation and Atomic Attachment of Nanocrystals in 2D Honeycomb Superlattices. Science 2014, 344, 1377-1380.

Alimoradi Jazi, M.; Janssen, V. A. E. C.; Evers, W. H.; Tadjine, A.; Delerue, C.; Siebbeles, L. D. A.; van der Zant, H. S. J.; Houtepen, A. J.; Vanmaekelbergh, D. Transport Properties of a Two-Dimensional PbSe Square Superstructure in an Electrolyte-Gated Transistor. Nano Lett. 2017, 17, 5238-5243.

Evers, W. H.; Schins, J. M.; Aerts, M.; Kulkarni, A.; Capiod, P.; Berthe, M.; Grandidier, B.; Delerue, C.; van der Zant, H. S. J.; van Overbeek, C.; et al. High Charge Mobility in Two-Dimensional Percolative Networks of PbSe Quantum Dots Connected by Atomic Bonds. Nat. Commun. 2015, 6, 8195.

Lu, H.; Carroll, G. M.; Chen, X.; Amarasinghe, D. K.; Neale, N. R.; Miller, E. M.; Sercel, P. C.; Rabuffetti, F. A.; Efros, A. L.; Beard, M. C. N-Type PbSe Quantum Dots via Post-Synthetic Indium Doping. J. Am. Chem. Soc. 2018, 140, 13753-13763.

Ramiro, I.; Kundu, B.; Dalmases, M.; Özdemir, O.; Pedrosa, M. Size- and Temperature-Dependent Intraband Optical Properties of Heavily n-Doped PbS Colloidal Quantum Dot Solid-State Films. ACS Nano 2020, 14, 7161-716.

Ramiro, I.; Özdemir, O.; Christodoulou, S.; Gupta, S.; Dalmases, M.; Torre, I.; Konstantatos, G. Mid- and Long-Wave Infrared Optoelectronics via Intraband Transitions in PbS Colloidal Quantum Dots. Nano Lett. 2020, 20, 1003-1008. 
(228) Bejaoui, A.; Alonso, M. I.; Garriga, M.; Campoy-Quiles, M.; Goñi, A. R.; Hetsch, F.; Kershaw, S. V.; Rogach, A. L.; To, C. H.; Foo, Y.; et al. Evaluation of the Dielectric Function of Colloidal Cd1-xHgxTe Quantum Dot Films by Spectroscopic Ellipsometry. Appl. Surf. Sci. 2017, 421, 295-300.

(229) Grynberg, M.; Le Toullec, R.; Balkanski, M. Dielectric Function in HgTe between 8 and 300 K. Phys. Rev. B 1974, 9, 517526.

(230) Szuszkiewicz, W.; Witowski, A. M.; Grynberg, M. The Dynamic Dielectric Function in HgSe and HgTe. Phys. Stat. Solidi B 1978, 87, 637-645.

(231) Houel, J.; Sauvage, S.; Boucaud, P.; Dazzi, A.; Prazeres, R.; Glotin, F.; Ortéga, J.-M.; Miard, A.; Lemaître, A. UltraweakAbsorption Microscopy of a Single Semiconductor Quantum Dot in the Midinfrared Range. Phys. Rev. Lett. 2007, 99, 217404.

(232) Peter, E.; Senellart, P.; Martrou, D.; Lemaître, A.; Hours, J.; Gérard, J. M.; Bloch, J. Exciton-Photon Strong-Coupling Regime for a Single Quantum Dot Embedded in a Microcavity. Phys. Rev. Lett. 2005, 95, 067401.

(233) Marzin, J.-Y.; Gérard, J.-M.; Izraël, A.; Barrier, D.; Bastard, G. Photoluminescence of Single InAs Quantum Dots Obtained by Self-Organized Growth on GaAs. Phys. Rev. Lett. 1994, 73, 716-719.

(234) Somaschi, N.; Giesz, V.; De Santis, L.; Loredo, J. C.; Almeida, M. P.; Hornecker, G.; Portalupi, S. L.; Grange, T.; Antón, C.; Demory, J.; et al. Near-Optimal Single-Photon Sources in the Solid State. Nat. Photonics 2016, 10, 340-345.

(235) Guenzer, C. S.; Bienenstock, A. Temperature Dependence of the HgTe Band Gap. Phys. Rev. B 1973, 8, 4655-4667.

(236) Dobrowolska, M.; Mycielski, A.; Dobrowolski, W. Determination of Temperature Dependence of Energy Gap in HgTe by Oscillatory Magnetotransmission Measurements. Solid State Commun. 1978, 27, 1233-1235.

(237) Guldner, Y.; Bastard, G.; Voos, M. Calculated Temperature Dependence of the Band Gap of HgTe-CdTe Superlattices. J. Appl. Phys. 1985, 57, 1403-1405.

(238) Olkhovets, A.; Hsu, R.-C.; Lipovskii, A.; Wise, F. W. Size-Dependent Temperature Variation of the Energy Gap in Lead-Salt Quantum Dots. Phys. Rev. Lett. 1998, 81, 3539-3542.

(239) Ramade, J.; Andriambariarijaona, L. M.; Steinmetz, V.; Goubet, N.; Legrand, L.; Barisien, T.; Bernardot, F.; Testelin, C.; Lhuillier, E.; Bramati, A.; et al. Exciton-Phonon Coupling in a $\mathrm{CsPbBr}_{3}$ Single Nanocrystal. Appl. Phys. Lett. 2018, 112, 072104.

(240) Livache, C.; Goubet, N.; Gréboval, C.; Martinez, B.; Ramade, J.; Qu, J.; Triboulin, A.; Cruguel, H.; Baptiste, B.; Klotz, S.; et al. Effect of Pressure on Interband and Intraband Transition of Mercury Chalcogenide Quantum Dots. J. Phys. Chem. $C$ 2019, 123, 13122-13130.

(241) Bernevig, B. A.; Hughes, T. L.; Zhang, S.-C. Quantum Spin Hall Effect and Topological Phase Transition in HgTe Quantum Wells. Science 2006, 314, 1757-1761.

(242) König, M.; Wiedmann, S.; Brüne, C.; Roth, A.; Buhmann, H.; Molenkamp, L. W.; Qi, X.-L.; Zhang, S.-C. Quantum Spin Hall Insulator State in HgTe Quantum Wells. Science 2007, 318, 766-770.

(243) Wu, G. Y.; McGill, T. C. Strain Effects in HgTe-CdTe Superlattices Grown on CdTe Substrates. Appl. Phys. Lett. 1985, 47, 634-636.

(244) Brüne, C.; Liu, C. X.; Novik, E. G.; Hankiewicz, E. M.; Buhmann, H.; Chen, Y. L.; Qi, X. L.; Shen, Z. X.; Zhang, S. C.; Molenkamp, L. W. Quantum Hall Effect from the Topological Surface States of Strained Bulk HgTe. Phys. Rev. Lett. 2011, 106, 126803.

(245) Wright, N. G.; McMahon, M. I.; Nelmes, R. J.; San-Miguel, A. Crystal Structure of the Cinnabar Phase of HgTe. Phys. Rev. $B$ 1993, 48, 13111-13114.

(246) San-Miguel, A.; Wright, N. G.; McMahon, M. I.; Nelmes, R. J. Pressure Evolution of the Cinnabar Phase of HgTe. Phys. Rev. B 1995, 51, 8731-8736.

(247) Werner, A.; Hochheimer, H. D.; Strössner, K.; Jayaraman, A. High-Pressure X-Ray Diffraction Studies on HgTe and HgS to 20 GPa. Phys. Rev. B 1983, 28, 3330-3334.

(248) Kafalas, J. A.; Gatos, H. C.; Lavine, M. C.; Banus, M. D. High Pressure Phase Transition in Mercury Selenide. J. Phys. Chem. Solids 1962, 23, 1541-1544.

(249) Ithurria, S.; Guyot-Sionnest, P.; Mahler, B.; Dubertret, B. $\mathrm{Mn}^{2+}$ as a Radial Pressure Gauge in Colloidal Core/Shell Nanocrystals. Phys. Rev. Lett. 2007, 99, 265501.

(250) Tolbert, S. H.; Alivisatos, A. P. Size Dependence of a First Order Solid-Solid Phase Transition: The Wurtzite to Rock Salt Transformation in CdSe Nanocrystals. Science 1994, 265, 373-376.

(251) Düz, I.; Erdem, I.; Kart, S. O.; Kuzucu, V. First Principles Investigations of HgX (X=S, Se and Te). Archives of Materials Science and Engineering 2016, 79, 5-11.

(252) Steiner, D.; Dorfs, D.; Banin, U.; Della Sala, F.; Manna, L.; Millo, O. Determination of Band Offsets in Heterostructured Colloidal Nanorods Using Scanning Tunneling Spectroscopy. Nano Lett. 2008, 8, 2954-2958.

(253) Diaconescu, B.; Padilha, L. A.; Nagpal, P.; Swartzentruber, B. S.; Klimov, V. I. Measurement of Electronic States of PbS Nanocrystal Quantum Dots Using Scanning Tunneling Spectroscopy: The Role of Parity Selection Rules in Optical Absorption. Phys. Rev. Lett. 2013, 110, 127406.

(254) Swart, I.; Liljeroth, P.; Vanmaekelbergh, D. Scanning Probe Microscopy and Spectroscopy of Colloidal Semiconductor Nanocrystals and Assembled Structures. Chem. Rev. 2016, 116, 11181-11219.

(255) Liljeroth, P.; Jdira, L.; Overgaag, K.; Grandidier, B.; Speller, S.; Vanmaekelbergh, D. Can Scanning Tunnelling Spectroscopy Measure the Density of States of Semiconductor Quantum Dots? Phys. Chem. Chem. Phys. 2006, 8, 3845-3850. 
(256) Overgaag, K.; Liljeroth, P.; Grandidier, B.; Vanmaekelbergh, D. Scanning Tunneling Spectroscopy of Individual PbSe Quantum Dots and Molecular Aggregates Stabilized in an Inert Nanocrystal Matrix. ACS Nano 2008, 2, 600-606.

(257) Jdira, L.; Liljeroth, P.; Stoffels, E.; Vanmaekelbergh, D.; Speller, S. Size-Dependent Single-Particle Energy Levels and Interparticle Coulomb Interactions in CdSe Quantum Dots Measured by Scanning Tunneling Spectroscopy. Phys. Rev. B 2006, 73, 115305.

(258) Banin, U.; Cao, Y.; Katz, D.; Millo, O. Identification of Atomic-like Electronic States in Indium Arsenide Nanocrystal Quantum Dots. Nature 1999, 400, 542-544.

(259) Wang, H.; Lhuillier, E.; Yu, Q.; Zimmers, A.; Dubertret, B.; Ulysse, C.; Aubin, H. Transport in a Single Self-Doped Nanocrystal. ACS Nano 2017, 11, 1222-1229.

(260) Janowitz, C.; Orlowski, N.; Manzke, R.; Golacki, Z. On the Band Structure of HgTe and HgSe - View from Photoemission. J. Alloys Compd. 2001, 328, 84-89.

(261) Bisquert, J.; Fabregat-Santiago, F.; Mora-Seró, I.; Garcia-Belmonte, G.; Barea, E. M.; Palomares, E. A Review of Recent Results on Electrochemical Determination of the Density of Electronic States of Nanostructured Metal-Oxide Semiconductors and Organic Hole Conductors. Inorganica Chim. Acta 2008, 361, 684-698.

(262) Livache, C.; Izquierdo, E.; Martinez, B.; Dufour, M.; Pierucci, D.; Keuleyan, S.; Cruguel, H.; Becerra, L.; Fave, J. L.; Aubin, H.; et al. Charge Dynamics and Optolectronic Properties in HgTe Colloidal Quantum Wells. Nano Lett. 2017, 17, 40674074.

(263) Spittel, D.; Poppe, J.; Meerbach, C.; Ziegler, C.; Hickey, S. G.; Eychmüller, A. Absolute Energy Level Positions in CdSe Nanostructures from Potential-Modulated Absorption Spectroscopy (EMAS). ACS Nano 2017, 11, 12174-12184.

(264) Haram, S. K.; Quinn, B. M.; Bard, A. J. Electrochemistry of CdS Nanoparticles: A Correlation between Optical and Electrochemical Band Gaps. J. Am. Chem. Soc. 2001, 123, 8860-8861.

(265) Sobrova, P.; Ryvolova, M.; Hubalek, J.; Adam, V.; Kizek, R. Voltammetry as a Tool for Characterization of CdTe Quantum Dots. Int. J. Mol. Sci. 2013, 14, 13497-13510.

(266) Liu, H.; Keuleyan, S.; Guyot-Sionnest, P. n- and p-Type HgTe Quantum Dot Films. J. Phys. Chem. C 2012, 116, $1344-1349$.

(267) Kroupa, D. M.; Vörös, M.; Brawand, N. P.; McNichols, B. W.; Miller, E. M.; Gu, J.; Nozik, A. J.; Sellinger, A.; Galli, G.; Beard, M. C. Tuning Colloidal Quantum Dot Band Edge Positions through Solution-Phase Surface Chemistry Modification. Nat. Commun. 2017, 8, 15257.

(268) Melnychuk, C.; Guyot-Sionnest, P. Slow Auger Relaxation in HgTe Colloidal Quantum Dots. J. Phys. Chem. Lett. 2018, 9, 2208-2211.

(269) Melnychuk, C.; Guyot-Sionnest, P. Auger Suppression in n-Type HgSe Colloidal Quantum Dots. ACS Nano 2019, 13, 1051210519.

(270) Leontiadou, M. A.; Al-Otaify, A.; Kershaw, S. V.; Zhovtiuk, O.; Kalytchuk, S.; Mott, D.; Maenosono, S.; Rogach, A. L.; Binks, D. J. Ultrafast Exciton Dynamics in $\mathrm{Cd}_{x} \mathrm{Hg}_{(1-\mathrm{x})}$ Te Alloy Quantum Dots. Chem. Phys. 2016, 469-470, 25-30.

(271) Liu, H.; Guyot-Sionnest, P. Photoluminescence Lifetime of Lead Selenide Colloidal Quantum Dots. J. Phys. Chem. C 2010, $114,14860-14863$.

(272) Robel, I.; Gresback, R.; Kortshagen, U.; Schaller, R. D.; Klimov, V. I. Universal Size-Dependent Trend in Auger Recombination in Direct-Gap and Indirect-Gap Semiconductor Nanocrystals. Phys. Rev. Lett. 2009, $102,177404$.

(273) Klimov, V. I.; McGuire, J. A.; Schaller, R. D.; Rupasov, V. I. Scaling of Multiexciton Lifetimes in Semiconductor Nanocrystals. Phys. Rev. B 2008, 77, 195324.

(274) Neppl, S.; Gessner, O. Time-Resolved X-Ray Photoelectron Spectroscopy Techniques for the Study of Interfacial Charge Dynamics. J. Electron Spectros. Relat. Phenomena 2015, 200, 64-77.

(275) Spencer, B. F.; Cliffe, M. J.; Graham, D. M.; Hardman, S. J. O.; Seddon, E. A.; Syres, K. L.; Thomas, A. G.; Sirotti, F.; Silly, M. G.; Akhtar, J.; et al. Chemically-Specific Time-Resolved Surface Photovoltage Spectroscopy: Carrier Dynamics at the Interface of Quantum Dots Attached to a Metal Oxide. Surf. Sci. 2015, 641, 320-325.

(276) Gréboval, C.; Rastogi, P.; Qu, J.; Chu, A.; Ramade, J.; Khalili, A.; Dabard, C.; Dang, T. H.; Cruguel, H.; Ouerghi, A.; et al. Time Resolved Photoemission to Unveil Electronic Coupling Between Absorbing and Transport Layers in a Quantum Dot Based Solar Cell, J. Phys. Chem. C 2020, 124, 23400-23409.

(277) Amelot, D.; Rastogi, P.; Martinez, B.; Gréboval, C.; Livache, C.; Bresciani, F. A.; Qu, J.; Chu, A.; Goyal, M.; Chee, S.-S.; et al. Revealing the Band Structure of FAPI Quantum Dot Film and Its Interfaces with Electron and Hole Transport Layer Using Time Resolved Photoemission. J. Phys. Chem. C 2020, 124, 3873-3880.

(278) Martinez, B.; Livache, C.; Goubet, N.; Jagtap, A.; Cruguel, H.; Ouerghi, A.; Lacaze, E.; Silly, M. G.; Lhuillier, E. Probing Charge Carrier Dynamics to Unveil the Role of Surface Ligands in HgTe Narrow Band Gap Nanocrystals. J. Phys. Chem. C 2018, $122,859-865$.

(279) Lhuillier, E.; Keuleyan, S.; Rekemeyer, P.; Guyot-Sionnest, P. Thermal Properties of Mid-Infrared Colloidal Quantum Dot Detectors. J. Appl. Phys. 2011, 110, 033110.

(280) Fidler, A. F.; Gao, J.; Klimov, V. I. Electron-Hole Exchange Blockade and Memory-Less Recombination in Photoexcited Films of Colloidal Quantum Dots. Nat. Phys. 2017, 13, 604-610.

(281) Gao, J.; Fidler, A. F.; Klimov, V. I. Carrier Multiplication Detected through Transient Photocurrent in Device-Grade Films of Lead Selenide Quantum Dots. Nat. Commun. 2015, 6, 8185.

(282) Chen, M.; Yu, H.; Kershaw, S. V.; Xu, H.; Gupta, S.; Hetsch, F.; Rogach, A. L.; Zhao, N. Fast, Air-Stable Infrared Photodetectors Based on Spray-Deposited Aqueous HgTe Quantum Dots. Adv. Funct. Mater. 2014, $24,53-59$. 
(283) Orenstein, J.; Kastner, M. A.; Vaninov, V. Transient Photoconductivity and Photo-Induced Optical Absorption in Amorphous Semiconductors. Philos. Mag. B 1982, 46, 23-62.

(284) Main, C.; Nesheva, D. Transient Photocurrent Techniques as a Means of Characterising Amorphous Semiconductors, J. Optoelectron. Adv. Mater. 2001, 3, 655-664.

(285) Orenstein, J.; Kastner, M. Photocurrent Transient Spectroscopy: Measurement of the Density of Localized States in a$\mathrm{As}_{2} \mathrm{Se}_{3}$. Phys. Rev. Lett. 1981, 46, 1421-1424.

(286) Guyot-Sionnest, P.; Lhuillier, E.; Liu, H. A Mirage Study of CdSe Colloidal Quantum Dot Films, Urbach Tail, and Surface States. J. Chem. Phys. 2012, 137, 154704.

(287) Guyot-Sionnest, P. Electrical Transport in Colloidal Quantum Dot Films. J. Phys. Chem. Lett. 2012, 3, 1169-1175.

(288) Koktysh, D. S.; Gaponik, N.; Reufer, M.; Crewett, J.; Scherf, U.; Eychmüller, A.; Lupton, J. M.; Rogach, A. L.; Feldmann, J. Near-Infrared Electroluminescence from HgTe Nanocrystals. Chem. Phys. Chem. 2004, 5, 1435-1438.

(289) O’Connor, É.; O’Riordan, A.; Doyle, H.; Moynihan, S.; Cuddihy, A.; Redmond, G. Near-Infrared Electroluminescent Devices Based on Colloidal HgTe Quantum Dot Arrays. Appl. Phys. Lett. 2005, 86, 201114.

(290) Rogach, A. L.; Koktysh, D. S.; Harrison, M.; Kotov, N. A. Layer-by-Layer Assembled Films of HgTe Nanocrystals with Strong Infrared Emission. Chem. Mater. 2000, 12, 1526-1528.

(291) Wen, Q.; Kershaw, S. V.; Kalytchuk, S.; Zhovtiuk, O.; Reckmeier, C.; Vasilevskiy, M. I.; Rogach, A. L. Impact of D2O/H2O Solvent Exchange on the Emission of HgTe and CdTe Quantum Dots: Polaron and Energy Transfer Effects. ACS Nano 2016, 10, 4301-4311.

(292) Gersten, J.; Nitzan, A. Spectroscopic Properties of Molecules Interacting with Small Dielectric Particles. J. Chem. Phys. 1981, 75, 1139-1152.

(293) van Driel, A. F.; Allan, G.; Delerue, C.; Lodahl, P.; Vos, W. L.; Vanmaekelbergh, D. Frequency-Dependent Spontaneous Emission Rate from CdSe and CdTe Nanocrystals: Influence of Dark States. Phys. Rev. Lett. 2005, 95, 236804.

(294) Klimov, V. I.; Mikhailovsky, A. A.; Xu, S.; Malko, A.; Hollingsworth, J. A.; Leatherdale, C. A.; Eisler, H.-J.; Bawendi, M. G. Optical Gain and Stimulated Emission in Nanocrystal Quantum Dots. Science 2000, 290, 314-317.

(295) Lim, J.; Park, Y.-S.; Klimov, V. I. Optical Gain in Colloidal Quantum Dots Achieved with Direct-Current Electrical Pumping. Nat. Mater. 2018, 17, 42-49.

(296) Roh, J.; Park, Y.-S.; Lim, J.; Klimov, V. I. Optically Pumped Colloidal-Quantum-Dot Lasing in LED-like Devices with an Integrated Optical Cavity. Nat. Commun. 2020, 11, 271.

(297) Geiregat, P.; Van Thourhout, D.; Hens, Z. A Bright Future for Colloidal Quantum Dot Lasers. NPG Asia Mater. 2019, 11, 41.

(298) Fan, F.; Voznyy, O.; Sabatini, R. P.; Bicanic, K. T.; Adachi, M. M.; McBride, J. R.; Reid, K. R.; Park, Y.-S.; Li, X.; Jain, A.; et al. Continuous-Wave Lasing in Colloidal Quantum Dot Solids Enabled by Facet-Selective Epitaxy. Nature 2017, 544, 75-79.

(299) Olk, P.; Buchler, B. C.; Sandoghdar, V.; Gaponik, N.; Eychmüller, A.; Rogach, A. L. Subwavelength Emitters in the NearInfrared Based on Mercury Telluride Nanocrystals. Appl. Phys. Lett. 2004, 84, 4732-4734.

(300) Shopova, S. I.; Farca, G.; Rosenberger, A. T.; Wickramanayake, W. M. S.; Kotov, N. A. Microsphere Whispering-GalleryMode Laser Using HgTe Quantum Dots. Appl. Phys. Lett. 2004, 85, 6101-6103.

(301) Guyot-Sionnest, P.; Roberts, J. A. Background Limited Mid-Infrared Photodetection with Photovoltaic HgTe Colloidal Quantum Dots. Appl. Phys. Lett. 2015, 107.

(302) Jagtap, A.; Goubet, N.; Livache, C.; Chu, A.; Martinez, B.; Gréboval, C.; Qu, J.; Dandeu, E.; Becerra, L.; Witkowski, N.; et al. Short Wave Infrared Devices Based on HgTe Nanocrystals with Air Stable Performances. J. Phys. Chem. C 2018, 122, 14979-14985.

(303) Ackerman, M. M.; Tang, X.; Guyot-Sionnest, P. Fast and Sensitive Colloidal Quantum Dot Mid-Wave Infrared Photodetectors. ACS Nano 2018, 12, 7264-7271.

(304) Tang, X.; Ackerman, M. M.; Shen, G.; Guyot-Sionnest, P. Towards Infrared Electronic Eyes: Flexible Colloidal Quantum Dot Photovoltaic Detectors Enhanced by Resonant Cavity. Small 2019, 15, 1804920.

(305) Pradhan, S.; Di Stasio, F.; Bi, Y.; Gupta, S.; Christodoulou, S.; Stavrinadis, A.; Konstantatos, G. High-Efficiency Colloidal Quantum Dot Infrared Light-Emitting Diodes via Engineering at the Supra-Nanocrystalline Level. Nat. Nanotechnol. 2019, 14, 72-79.

(306) Chuang, C.-H. M.; Brown, P. R.; Bulović, V.; Bawendi, M. G. Improved Performance and Stability in Quantum Dot Solar Cells through Band Alignment Engineering. Nat. Mater. 2014, 13, 796-801.

(307) Roither, J.; Kovalenko, M. V.; Pichler, S.; Schwarzl, T.; Heiss, W. Nanocrystal-Based Microcavity Light-Emitting Devices Operating in the Telecommunication Wavelength Range. Appl. Phys. Lett. 2005, 86, 241104.

(308) Wang, C.; Roither, J.; Kirschschlager, R.; Kovalenko, M. V.; Brehm, M.; Fromherz, T.; Kan, Q.; Tan, P.; Liu, J.; Chen, H.; et al. Enhanced Infrared Emission from Colloidal HgTe Nanocrystal Quantum Dots on Silicon-on-Insulator Photonic Crystals. Appl. Phys. Lett. 2009, 95, 053107.

(309) Chen, T.; Reich, K. V.; Kramer, N. J.; Fu, H.; Kortshagen, U. R.; Shklovskii, B. I. Metal-Insulator Transition in Films of Doped Semiconductor Nanocrystals. Nat. Mater. 2016, 15, 299-303.

(310) Fu, H.; Reich, K. V.; Shklovskii, B. I. Hopping Conductivity and Insulator-Metal Transition in Films of Touching Semiconductor Nanocrystals. Phys. Rev. B 2016, 93, 125430.

(311) MOTT, N. F. Metal-Insulator Transition. Rev. Mod. Phys. 1968, 40, 677-683. 
(312) Moreira, H.; Yu, Q.; Nadal, B.; Bresson, B.; Rosticher, M.; Lequeux, N.; Zimmers, A.; Aubin, H. Electron Cotunneling Transport in Gold Nanocrystal Arrays. Phys. Rev. Lett. 2011, 107, 176803.

(313) Liu, Y.; Gibbs, M.; Puthussery, J.; Gaik, S.; Ihly, R.; Hillhouse, H. W.; Law, M. Dependence of Carrier Mobility on Nanocrystal Size and Ligand Length in PbSe Nanocrystal Solids. Nano Lett. 2010, 10, 1960-1969.

(314) Kim, H.; Cho, K.; Kim, D.-W.; Lee, H.-R.; Kim, S. Bottom- and Top-Gate Field-Effect Thin-Film Transistors with p Channels of Sintered HgTe Nanocrystals. Appl. Phys. Lett. 2006, 89, 173107.

(315) Kim, D.-W.; Jang, J.; Kim, H.; Cho, K.; Kim, S. Electrical Characteristics of HgTe Nanocrystal-Based Thin Film Transistors Fabricated on Flexible Plastic Substrates. Thin Solid Films 2008, 516, 7715-7719.

(316) Lan, X.; Chen, M.; Hudson, M. H.; Kamysbayev, V.; Wang, Y.; Guyot-Sionnest, P.; Talapin, D. V. Quantum Dot Solids Showing State-Resolved Band-like Transport. Nat. Mater. 2020, 19, 323-329.

(317) Chen, M.; Shen, G.; Guyot-Sionnest, P. State-Resolved Mobility of $1 \mathrm{~cm}^{2} /(\mathrm{Vs})$ with HgSe Quantum Dot Films. J. Phys. Chem. Lett. 2020, 11, 2303-2307.

(318) Gréboval, C.; Noumbe, U.; Goubet, N.; Livache, C.; Ramade, J.; Qu, J.; Chu, A.; Martinez, B.; Prado, Y.; Ithurria, S.; et al. Field-Effect Transistor and Photo-Transistor of Narrow-Band-Gap Nanocrystal Arrays Using lonic Glasses. Nano Lett. 2019, 19, 3981-3986.

(319) Kovalenko, M. V.; Schaller, R. D.; Jarzab, D.; Loi, M. A.; Talapin, D. V. Inorganically Functionalized PbS-CdS Colloidal Nanocrystals: Integration into Amorphous Chalcogenide Glass and Luminescent Properties. J. Am. Chem. Soc. 2012, 134, 2457-2460.

(320) Yakunin, S.; Dirin, D. N.; Protesescu, L.; Sytnyk, M.; Tollabimazraehno, S.; Humer, M.; Hackl, F.; Fromherz, T.; Bodnarchuk, M. I.; Kovalenko, M. V.; et al. High Infrared Photoconductivity in Films of Arsenic-Sulfide-Encapsulated Lead-Sulfide Nanocrystals. ACS Nano 2014, 8, 12883-12894.

(321) Lambert, K.; Dendooven, J.; Detavernier, C.; Hens, Z. Embedding Quantum Dot Monolayers in $\mathrm{Al}_{2} \mathrm{O}_{3} \mathrm{Using} \mathrm{Atomic}^{2} \mathrm{Layer}$ Deposition. Chem. Mater. 2011, 23, 126-128.

(322) Devloo-Casier, K.; Geiregat, P.; Ludwig, K. F.; van Stiphout, K.; Vantomme, A.; Hens, Z.; Detavernier, C.; Dendooven, J. A Case Study of ALD Encapsulation of Quantum Dots: Embedding Supported CdSe/CdS/ZnS Quantum Dots in a ZnO Matrix. J. Phys. Chem. C 2016, 120, 18039-18045.

(323) Pourret, A.; Guyot-Sionnest, P.; Elam, J. W. Atomic Layer Deposition of ZnO in Quantum Dot Thin Films. Adv. Mater. 2009, 21, 232-235.

(324) Liu, Y.; Tolentino, J.; Gibbs, M.; Ihly, R.; Perkins, C. L.; Liu, Y.; Crawford, N.; Hemminger, J. C.; Law, M. PbSe Quantum Dot Field-Effect Transistors with Air-Stable Electron Mobilities above $7 \mathrm{Cm}^{2} \mathrm{~V}^{-1} \mathrm{~s}^{-1}$. Nano Lett. 2013, 13, $1578-1587$.

(325) Lhuillier, E.; Keuleyan, S.; Guyot-Sionnest, P. Transport Properties of Mid-Infrared Colloidal Quantum Dot Films. In Quantum Dots and Nanostructures: Synthesis, Characterization, and Modeling IX; International Society for Optics and Photonics, 2012, 8271, 827109.

(326) Baibich, M. N.; Broto, J. M.; Fert, A.; Van Dau, F. N.; Petroff, F.; Etienne, P.; Creuzet, G.; Friederich, A.; Chazelas, J. Giant Magnetoresistance of (001)Fe/(001)Cr Magnetic Superlattices. Phys. Rev. Lett. 1988, 61, 2472-2475.

(327) Cryer, M. E.; Fiedler, H.; Halpert, J. E. Photo-Electrosensitive Memristor Using Oxygen Doping in HgTe Nanocrystal Films. ACS Appl. Mater. Interfaces 2018, 10, 18927-18934.

(328) Jang, J.; Liu, W.; Son, J. S.; Talapin, D. V. Temperature-Dependent Hall and Field-Effect Mobility in Strongly Coupled AllInorganic Nanocrystal Arrays. Nano Lett. 2014, 14, 653-662.

(329) Yun, J.; Cho, K.; Kim, S. Flexible Logic Circuits Composed of Chalcogenide-Nanocrystal-Based Thin Film Transistors. Nanotechnology 2010, 21, 235204.

(330) Chen, M.; Lu, H.; Abdelazim, N. M.; Zhu, Y.; Wang, Z.; Ren, W.; Kershaw, S. V.; Rogach, A. L.; Zhao, N. Mercury Telluride Quantum Dot Based Phototransistor Enabling High-Sensitivity Room-Temperature Photodetection at $2000 \mathrm{~nm}$. ACS Nano 2017, 11, 5614-5622.

(331) Kang, M. S.; Sahu, A.; Norris, D. J.; Frisbie, C. D. Size-Dependent Electrical Transport in CdSe Nanocrystal Thin Films. Nano Lett. 2010, 10, 3727-3732.

(332) Wang, C.; Shim, M.; Guyot-Sionnest, P. Electrochromic Nanocrystal Quantum Dots. Science 2001, $291,2390-2392$.

(333) Vanmaekelbergh, D.; Liljeroth, P. Electron-Conducting Quantum Dot Solids: Novel Materials Based on Colloidal Semiconductor Nanocrystals. Chem. Soc. Rev. 2005, 34, 299-312.

(334) Puntambekar, A.; Wang, Q.; Miller, L.; Smieszek, N.; Chakrapani, V. Electrochemical Charging of CdSe Quantum Dots: Effects of Adsorption versus Intercalation. ACS Nano 2016, 10, 10988-10999.

(335) Biscaras, J.; Chen, Z.; Paradisi, A.; Shukla, A. Onset of Two-Dimensional Superconductivity in Space Charge Doped FewLayer Molybdenum Disulfide. Nat. Commun. 2015, 6, 8826.

(336) Wu, C.-L.; Yuan, H.; Li, Y.; Gong, Y.; Hwang, H. Y.; Cui, Y. Gate-Induced Metal-Insulator Transition in MoS2 by Solid Superionic Conductor $\mathrm{LaF}_{3}$. Nano Lett. 2018, 18, 2387-2392.

(337) Rastogi, P.; Chu, A.; Greboval, C.; Qu, J.; Noumbe, U. N.; Chee, S.-S.; Goyal, M.; Khalili Lazarjani, A.; Xu, X. Z.; Cruguel, H.; et al. Pushing Absorption of Perovskite Nanocrystals into the Infrared. Nano Lett. 2020, 20, 3999-4006.

(338) Noumbé, U. N.; Gréboval, C.; Livache, C.; Brule, T.; Doudin, B.; Ouerghi, A.; Lhuillier, E.; Dayen, J.-F. Ionic Glass-Gated 2D Material-Based Phototransistor: MoSez over LaF 3 as Case Study. Adv. Funct. Mater. 2019, 29, 1902723. 
(339) Gréboval, C.; Chu, A.; Magalhaes, D.V.; Ramade, J.; Qu, J.; Rastogi, P.; Khalili, A.; Chee, S.-S.; Aubin, A.; Vincent, G.; et al. Ferroelectric Gating of Narrow Band-Gap Nanocrystal Arrays with Enhanced Light Matter Coupling, ACS Photonics 2021, 8, 259-268.

(340) Biscaras, J.; Hurand, S.; Feuillet-Palma, C.; Rastogi, A.; Budhani, R. C.; Reyren, N.; Lesne, E.; Lesueur, J.; Bergeal, N. Limit of the Electrostatic Doping in Two-Dimensional Electron Gases of $\mathrm{LaxO}_{3}(\mathrm{X}=\mathrm{Al}, \mathrm{Ti}) / \mathrm{SrTiO}_{3}$. Sci. Rep. 2014, 4, 6788.

(341) Caviglia, A. D.; Gariglio, S.; Reyren, N.; Jaccard, D.; Schneider, T.; Gabay, M.; Thiel, S.; Hammerl, G.; Mannhart, J.; Triscone, J.-M. Electric Field Control of the $\mathrm{LaAlO}_{3} / \mathrm{SrTiO}_{3}$ Interface Ground State. Nature 2008, 456, 624-627.

(342) Biscaras, J.; Bergeal, N.; Hurand, S.; Grossetête, C.; Rastogi, A.; Budhani, R. C.; LeBoeuf, D.; Proust, C.; Lesueur, TwoDimensional Superconducting Phase in $\mathrm{LaTiO}_{3} / \mathrm{SrTiO}_{3}$ Heterostructures Induced by High-Mobility Carrier Doping. Phys. Rev. Lett. 2012, 108, 247004.

(343) Yu, D.; Wang, C.; Guyot-Sionnest, P. N-Type Conducting CdSe Nanocrystal Solids. Science 2003, 300, 1277-1280.

(344) Yu, Q.; Cui, L.; Lequeux, N.; Zimmers, A.; Ulysse, C.; Rebuttini, V.; Pinna, N.; Aubin, H. In-Vacuum Projection of Nanoparticles for On-Chip Tunneling Spectroscopy. ACS Nano 2013, 7, 1487-1494.

(345) Yu, Q.; Mottaghizadeh, A.; Wang, H.; Ulysse, C.; Zimmers, A.; Rebuttini, V.; Pinna, N.; Aubin, H. Verwey Transition in Single Magnetite Nanoparticles. Phys. Rev. B 2014, 90, 075122.

(346) Wang, H.; Lhuillier, E.; Yu, Q.; Mottaghizadeh, A.; Ulysse, C.; Zimmers, A.; Descamps-Mandine, A.; Dubertret, B.; Aubin, H. Effects of Electron-Phonon Interactions on the Electron Tunneling Spectrum of PbS Quantum Dots. Phys. Rev. B 2015, 92, 041403.

(347) Gravrand, O.; Wlassow, J.; Bonnefond, L. A Calibration Method for the Measurement of IR Detector Spectral Responses Using a FTIR Spectrometer Equipped with a DTGS Reference Cell. In High Energy, Optical, and Infrared Detectors for Astronomy VI; International Society for Optics and Photonics. 2014, 9154, 915420.

(348) Liu, H.; Lhuillier, E.; Guyot-Sionnest, P. 1/f Noise in Semiconductor and Metal Nanocrystal Solids. J. Appl. Phys. 2014, 115, 154309.

(349) Lai, Y.; Li, H.; Kim, D. K.; Diroll, B. T.; Murray, C. B.; Kagan, C. R. Low-Frequency (1/f) Noise in Nanocrystal Field-Effect Transistors. ACS Nano 2014, 8, 9664-9672.

(350) Hooge, F. N. 1/f Noise Sources. IEEE Trans. Electron Devices 1994, 41, 1926-1935.

(351) Downs, C.; Vandervelde, T. E. Progress in Infrared Photodetectors Since 2000. Sensors 2013, 13, 5054-5098.

(352) Konstantatos, G.; Sargent, E. H. PbS Colloidal Quantum Dot Photoconductive Photodetectors: Transport, Traps, and Gain. Appl. Phys. Lett. 2007, 91, 173505.

(353) Prins, F.; Buscema, M.; Seldenthuis, J. S.; Etaki, S.; Buchs, G.; Barkelid, M.; Zwiller, V.; Gao, Y.; Houtepen, A. J.; Siebbeles, L. D. A.; et al. Fast and Efficient Photodetection in Nanoscale Quantum-Dot Junctions. Nano Lett. 2012, 12, 5740-5743.

(354) Dong, Y.; Chen, M.; Yiu, W. K.; Zhu, Q.; Zhou, G.; Kershaw, S. V.; Ke, N.; Wong, C. P.; Rogach, A. L.; Zhao, N. Solution Processed Hybrid Polymer: HgTe Quantum Dot Phototransistor with High Sensitivity and Fast Infrared Response up to $2400 \mathrm{Nm}$ at Room Temperature. Adv. Sci. 2020, 7, 2000068.

(355) Schornbaum, J.; Zakharko, Y.; Held, M.; Thiemann, S.; Gannott, F.; Zaumseil, J. Light-Emitting Quantum Dot Transistors: Emission at High Charge Carrier Densities. Nano Lett. 2015, 15, 1822-1828.

(356) Hotta, S.; Yamao, T.; Bisri, S. Z.; Takenobu, T.; Iwasa, Y. Organic Single-Crystal Light-Emitting Field-Effect Transistors. J. Mater. Chem. C 2014, 2, 965-980.

(357) Shulga, A. G.; Kahmann, S.; Dirin, D. N.; Graf, A.; Zaumseil, J.; Kovalenko, M. V.; Loi, M. A. Electroluminescence Generation in PbS Quantum Dot Light-Emitting Field-Effect Transistors with Solid-State Gating. ACS Nano 2018, 12, 12805-12813.

(358) Kahmann, S.; Shulga, A.; Loi, M. A. Quantum Dot Light-Emitting Transistors-Powerful Research Tools and Their Future Applications. Adv. Funct. Mater. 2019, 30, 1904174.

(359) Bisri, S. Z.; Takenobu, T.; Sawabe, K.; Tsuda, S.; Yomogida, Y.; Yamao, T.; Hotta, S.; Adachi, C.; Iwasa, Y. p-i-n Homojunction in Organic Light-Emitting Transistors. Adv. Mater. 2011, 23, 2753-2758.

(360) Liu, C.-F.; Liu, X.; Lai, W.-Y.; Huang, W. Organic Light-Emitting Field-Effect Transistors: Device Geometries and Fabrication Techniques. Adv. Mater. 2018, 30, 1802466.

(361) He, P.; Jiang, C.; Lan, L.; Sun, S.; Li, Y.; Gao, P.; Zhang, P.; Dai, X.; Wang, J.; Peng, J.; et al. High-Performance, SolutionProcessed Quantum Dot Light-Emitting Field-Effect Transistors with a Scandium-Incorporated Indium Oxide Semiconductor. ACS Nano 2018, 12, 4624-4629.

(362) Kagan, C. R. Flexible Colloidal Nanocrystal Electronics. Chem. Soc. Rev. 2019, 48, 1626-1641.

(363) Jang, J.; Cho, K.; Lee, S. H.; Kim, S. Transparent and Flexible Thin-Film Transistors with Channel Layers Composed of Sintered HgTe Nanocrystals. Nanotechnology 2007, 19, 015204.

(364) Hao, Q.; Tang, X.; Cheng, Y.; Hu, Y. Development of Flexible and Curved Infrared Detectors with HgTe Colloidal Quantum Dots. Infrared Phys. Technol. 2020, 108, 103344.

(365) Diroll, B. T.; Chen, M.; Coropceanu, I.; Williams, K. R.; Talapin, D. V.; Guyot-Sionnest, P.; Schaller, R. D. Polarized NearInfrared Intersubband Absorptions in CdSe Colloidal Quantum Wells. Nat. Commun. 2019, 10, 4511.

(366) Guyot-Sionnest, P.; Hines, M. A. Intraband Transitions in Semiconductor Nanocrystals. Appl. Phys. Lett. 1998, 72, 686688.

(367) Gendron, L.; Carras, M.; Huynh, A.; Ortiz, V.; Koeniguer, C.; Berger, V. Quantum Cascade Photodetector. Appl. Phys. Lett. 2004, 85, 2824-2826. 
(368) Lhuillier, E.; Ribet-Mohamed, I.; Rosencher, E.; Patriarche, G.; Buffaz, A.; Berger, V.; Carras, M. Interface Roughness Transport in Terahertz Quantum Cascade Detectors. Appl. Phys. Lett. 2010, 96, 061111.

(369) Delga, A. 8 - Quantum Cascade Detectors: A Review. In Mid-infrared Optoelectronics; Tournié, E., Cerutti, L., Eds.; Woodhead Publishing Series in Electronic and Optical Materials; Woodhead Publishing, 2020, 337-377.

(370) Konstantatos, G.; Badioli, M.; Gaudreau, L.; Osmond, J.; Bernechea, M.; de Arquer, F. P. G.; Gatti, F.; Koppens, F. H. L. Hybrid Graphene-Quantum Dot Phototransistors with Ultrahigh Gain. Nat. Nanotechnol. 2012, 7, 363-368.

(371) Sun, Z.; Liu, Z.; Li, J.; Tai, G.; Lau, S.-P.; Yan, F. Infrared Photodetectors Based on CVD-Grown Graphene and PbS Quantum Dots with Ultrahigh Responsivity. Adv. Mater. 2012, 24, 5878-5883.

(372) Jariwala, D.; Marks, T. J.; Hersam, M. C. Mixed-Dimensional van Der Waals Heterostructures. Nat. Mater. 2017, 16, 170181.

(373) Grotevent, M. J.; Hail, C. U.; Yakunin, S.; Dirin, D. N.; Thodkar, K.; Barin, G. B.; Guyot-Sionnest, P.; Calame, M.; Poulikakos, D.; Kovalenko, M. V.; et al. Nanoprinted Quantum Dot-Graphene Photodetectors. Adv. Opt. Mater. 2019, 7, 1900019.

(374) Robin, A.; Lhuillier, E.; Xu, X. Z.; Ithurria, S.; Aubin, H.; Ouerghi, A.; Dubertret, B. Engineering the Charge Transfer in All 2D Graphene-Nanoplatelets Heterostructure Photodetectors. Sci. Rep. 2016, 6, 24909.

(375) Tang, X.; Lai, K. W. C. Graphene/HgTe Quantum-Dot Photodetectors with Gate-Tunable Infrared Response. ACS Appl. Nano Mater. 2019, 2, 6701-6706.

(376) Tang, X.; Wu, G. F.; Lai, K. W. C. Twisted Graphene-Assisted Photocarrier Transfer from HgSe Colloidal Quantum Dots into Silicon with Enhanced Collection and Transport Efficiency. Appl. Phys. Lett. 2017, 110, 241104.

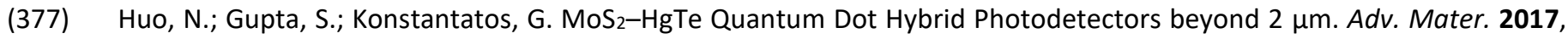
29, 1606576.

(378) Balandin, A. A. Low-Frequency $1 / f$ Noise in Graphene Devices. Nat. Nanotechnol. 2013, 8, 549-555.

(379) Nam, M.; Kim, S.; Kim, S.; Jeong, S.; Kim, S. W.; Lee, K. Near-Infrared-Sensitive Bulk Heterojunction Solar Cells Using Nanostructured Hybrid Composites of HgTe Quantum Dots and a Low-Bandgap Polymer. Sol. Energy Mater. Sol. Cells 2014, 126, 163-169.

(380) Im, S. H.; Kim, H.; Kim, S. W.; Kim, S.-W.; Seok, S. II. Efficient HgTe Colloidal Quantum Dot-Sensitized near-Infrared Photovoltaic Cells. Nanoscale 2012, 4, 1581.

(381) Sun, L.; Shen, L.; Meng, F.; Xu, P.; Guo, W.; Ruan, S. The Short Circuit Current Improvement in P3HT:PCBM Based Polymer Solar Cell by Introducing PSBTBT as Additional Electron Donor. J. Nanosci. Nanotechnol. 2014, 14, 3446-3449.

(382) Grätzel, M. Dye-Sensitized Solar Cells. J. Photochem. Photobio. C: Photochemistry Rev. 2003, 4, $145-153$.

(383) Baxter, J. B.; Aydil, E. S. Nanowire-Based Dye-Sensitized Solar Cells. Appl. Phys. Lett. 2005, 86, 053114.

(384) Savich, G. R.; Pedrazzani, J. R.; Sidor, D. E.; Maimon, S.; Wicks, G. W. Dark Current Filtering in Unipolar Barrier Infrared Detectors. Appl. Phys. Lett. 2011, 99, 121112.

(385) Savich, G. R.; Pedrazzani, J. R.; Sidor, D. E.; Wicks, G. W. Benefits and Limitations of Unipolar Barriers in Infrared Photodetectors. Infrared Phys. Technol. 2013, 59, 152-155.

(386) Dolzhnikov, D. S.; Zhang, H.; Jang, J.; Son, J. S.; Panthani, M. G.; Shibata, T.; Chattopadhyay, S.; Talapin, D. V. CompositionMatched Molecular "Solders" for Semiconductors. Science 2015, 347, 425-428.

(387) Palaferri, D.; Todorov, Y.; Bigioli, A.; Mottaghizadeh, A.; Gacemi, D.; Calabrese, A.; Vasanelli, A.; Li, L.; Davies, A. G.; Linfield, E. H.; et al. Room-Temperature Nine-Mm-Wavelength Photodetectors and GHz-Frequency Heterodyne Receivers. Nature 2018, 556, 85-88.

(388) Zhu, B.; Chen, M.; Zhu, Q.; Zhou, G.; Abdelazim, N. M.; Zhou, W.; Kershaw, S. V.; Rogach, A. L.; Zhao, N.; Tsang, H. K. Integrated Plasmonic Infrared Photodetector Based on Colloidal HgTe Quantum Dots. Adv. Mater. Technol. 2019, 4, 1900354.

(389) Chen, M.; Shao, L.; Kershaw, S. V.; Yu, H.; Wang, J.; Rogach, A. L.; Zhao, N. Photocurrent Enhancement of HgTe Quantum Dot Photodiodes by Plasmonic Gold Nanorod Structures. ACS Nano 2014, 8, 8208-8216.

(390) Tang, X.; Chen, M.; Ackerman, M. M.; Melnychuk, C.; Guyot-Sionnest, P. Direct Imprinting of Quasi-3D Nanophotonic Structures into Colloidal Quantum-Dot Devices. Adv. Mater. 2020, 32, 1906590.

(391) Chen, H.-L.; Cattoni, A.; De Lépinau, R.; Walker, A. W.; Höhn, O.; Lackner, D.; Siefer, G.; Faustini, M.; Vandamme, N.; Goffard, J.; et al. A 19.9\%-Efficient Ultrathin Solar Cell Based on a 205-nm-Thick GaAs Absorber and a Silver Nanostructured Back Mirror. Nat. Energy 2019, 4, 761-767.

(392) Rogalski, A. Dual-Band Infrared Detectors. In Photodetectors: Materials and Devices V; International Society for Optics and Photonics, 2000, 3948, 17-30.

(393) Choi, J. J.; Wenger, W. N.; Hoffman, R. S.; Lim, Y.-F.; Luria, J.; Jasieniak, J.; Marohn, J. A.; Hanrath, T. Solution-Processed Nanocrystal Quantum Dot Tandem Solar Cells. Adv. Mater. 2011, 23, 3144-3148.

(394) Péré-Laperne, N.; Rubaldo, L.; Kerlain, A.; Carrère, E.; Dargent, L.; Taalat, R.; Berthoz, J. 10 um Pitch Design of HgCdTe Diode Array in Sofradir. In Quantum Sensing and Nanophotonic Devices XII; International Society for Optics and Photonics. 2015, 9370, 937022.

(395) Livache, C.; Martinez, B.; Goubet, N.; Ramade, J.; Lhuillier, E. Road Map for Nanocrystal Based Infrared Photodetectors. Front. Chem. 2018, 6.

(396) Na, J.; Park, K.; Kim, J. T.; Choi, W. K.; Song, Y.-W. Air-Stable Few-Layer Black Phosphorus Phototransistor for near-Infrared Detection. Nanotechnology 2017, 28, 085201. 
(397) Amani, M.; Regan, E.; Bullock, J.; Ahn, G. H.; Javey, A. Mid-Wave Infrared Photoconductors Based on Black PhosphorusArsenic Alloys. ACS Nano 2017, 11, 11724-11731.

(398) Huang, L.; Tan, W. C.; Wang, L.; Dong, B.; Lee, C.; Ang, K.-W. Infrared Black Phosphorus Phototransistor with Tunable Responsivity and Low Noise Equivalent Power. ACS Appl. Mater. Interfaces 2017, 9, 36130-36136.

(399) Suess, R. J.; Leong, E.; Garrett, J. L.; Zhou, T.; Salem, R.; Munday, J. N.; Murphy, T. E.; Mittendorff, M. Mid-Infrared TimeResolved Photoconduction in Black Phosphorus. 2D Mater. 2016, 3, 041006.

(400) Guo, Q.; Pospischil, A.; Bhuiyan, M.; Jiang, H.; Tian, H.; Farmer, D.; Deng, B.; Li, C.; Han, S.-J.; Wang, H.; et al. Black Phosphorus Mid-Infrared Photodetectors with High Gain. Nano Lett. 2016, 16, 4648-4655.

(401) Wu, D.; Wang, Y.; Zeng, L.; Jia, C.; Wu, E.; Xu, T.; Shi, Z.; Tian, Y.; Li, X.; Tsang, Y. H. Design of 2D Layered PtSe 2 Heterojunction for the High-Performance, Room-Temperature, Broadband, Infrared Photodetector. ACS Photonics 2018, 5, 3820-3827.

(402) Yu, X.; Yu, P.; Wu, D.; Singh, B.; Zeng, Q.; Lin, H.; Zhou, W.; Lin, J.; Suenaga, K.; Liu, Z.; et al. Atomically Thin Noble Metal Dichalcogenide: A Broadband Mid-Infrared Semiconductor. Nat. Commun. 2018, 9, 1545.

(403) Long, M.; Wang, Y.; Wang, P.; Zhou, X.; Xia, H.; Luo, C.; Huang, S.; Zhang, G.; Yan, H.; Fan, Z.; et al. Palladium Diselenide Long-Wavelength Infrared Photodetector with High Sensitivity and Stability. ACS Nano 2019, 13, $2511-2519$.

(404) Luo, L.-B.; Wang, D.; Xie, C.; Hu, J.-G.; Zhao, X.-Y.; Liang, F.-X. PdSe2 Multilayer on Germanium Nanocones Array with Light Trapping Effect for Sensitive Infrared Photodetector and Image Sensing Application. Adv. Funct. Mater. 2019, 29, 1900849.

(405) Sahu, A.; Khare, A.; Deng, D. D.; Norris, D. J. Quantum Confinement in Silver Selenide Semiconductor Nanocrystals. Chem. Commun. 2012, 48, 5458-5460.

(406) Sahu, A.; Qi, L.; Kang, M. S.; Deng, D.; Norris, D. J. Facile Synthesis of Silver Chalcogenide $\left(\mathrm{Ag}_{2} \mathrm{E}\right.$; $\left.\mathrm{E}=\mathrm{Se}, \mathrm{S}, \mathrm{Te}\right)$ Semiconductor Nanocrystals. J. Am. Chem. Soc. 2011, 133, 6509-6512.

(407) Richner, P.; Galliker, P.; Lendenmann, T.; Kress, S. J. P.; Kim, D. K.; Norris, D. J.; Poulikakos, D. Full-Spectrum Flexible Color Printing at the Diffraction Limit. ACS Photonics 2016, 3, 754-757.

(408) Richner, P.; Kress, S. J. P.; Norris, D. J.; Poulikakos, D. Charge Effects and Nanoparticle Pattern Formation in Electrohydrodynamic NanoDrip Printing of Colloids. Nanoscale 2016, 8, 6028-6034.

(409) Rinnerbauer, V.; Kovalenko, M.; Lavchiev, V.; Kocher, G.; Roither, J.; Heiss, W.; Hingerl, K. Spectroscopic Ellipsometry of Layer by Layer Deposited Colloidal HgTe Nanocrystals Exhibiting Quantum Confinement. Physica E Low Dimens. Syst. Nanostruct. 2006, 32, 104-107.

(410) Rinnerbauer, V.; Hingerl, K.; Kovalenko, M.; Heiss, W. Quantum Confinement in Layer-by-Layer Deposited Colloidal HgTe Nanocrystals Determined by Spectroscopic Ellipsometry. Appl. Surf. Sci. 2007, 254, 291-294. 